\title{
Atlas Breached Waste Package and Drip Shield Experiments: Breached Drip Shield Tests
}

\author{
By \\ Zane P. Walton
}

Prepared for:

U.S. Department of Energy

Office of Repository Development

P.O. Box 364629

North Las Vegas, Nevada 89036-0307

Prepared by:

Bechtel SAIC Company LLC

1180 Town Center Drive

Las Vegas, Nevada 89144

Under Contract Number

DE-AC08-01RW12101 


\section{DISCLAIMER}

This report was prepared as an account of work sponsored by an agency of the United States Government. Neither the United States Government nor any agency thereof, nor any of their employees, nor any of their contractors, subcontractors or their employees, makes any warranty, express or implied or assumes any legal liability or responsibility for the accuracy, completeness, or any third party's use or the results of such use of any information, apparatus, product, or process disclosed, or represents that its use would not infringe privately owned rights. Reference herein to any specific commercial product, process, or service by trace name, trademark, manufacturer, or otherwise, does not necessarily constitute or imply its endorsement, recommendation, or favoring by the United States Government or any agency thereof or its contractors or subcontractors. The views and opinions of authors expressed herein do not necessarily state or reflect those of the United States Government or any agency thereof. 
Originator:

Zne P. Watte

Z.P. Walton

Science \& Engineering Associates, Inc.

Checker:

H. N. Kalia

Cle

Test Coordination Office, EBS Testing

QE Reviewer:

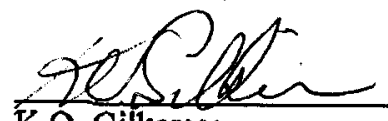

K.o. Gilkerson

QA Science Products

Responsible Manager approval:

mecoul setulh for

C. L. Howard

EBS/ Geoengineering Testing Department $\frac{5 / 28 / 2003}{\text { Date }}$

$\frac{5 / 28 / 2003}{\text { Date }}$ $\frac{5 / 28 / 2003}{\text { Date }}$ 


\section{EXECUTIVE SUMMARY}

The Engineered Barrier System (EBS) represents one system in the performance of the Yucca Mountain high-level radioactive waste (HLW) repository to isolate and prevent the transport of radionuclides from the site to the accessible environment. Breached Waste Package and Drip Shield Experiments (BWPDSE) were performed at the Department of Energy's National Nuclear Security Administration Nevada Support Facility in North Las Vegas, NV in the A-1 lowbay between May 2, 2002 and July 25, 2002. Data collected from the BWPDSE will be used to support the flux splitting model used in Analysis and Modeling Report ANL-WIS-PA-000001 REV 00 ICN 03 EBS Radionuclide Transport Abstraction (BSC 2001a). Tests were conducted by dripping water from heights representing the drift crown or wall on a full-scale section of a drip shield with both smooth and rough surfaces. The drip shields had machined square breaches that represent the general corrosion breaches or nodes in the WAPDEG Analysis of Waste Package and Drip Shield Degradation AMR (CRWMS M\&O 2000d). Tests conducted during the BWPDSE included: initial tests to determine the splash radius distances and spread factor from the line of drip impact, single patch tests to determine the amount of water collected in target breaches from splashing or rivulet flow, multiple patch tests to determine the amount of water collected in several breaches from both splashing and rivulet flow, and bounding flow rate tests. Supplemental data were collected to provide additional information for rivulet spread, pan evaporation in the test chamber, and water temperatures of the input water and drip shield surface water. The primary flow mechanism observed on both smooth and rough surfaces was rivulet flow, not film flow. Lateral rivulet spread distances were, in general, wider on the smooth drip shield surface than on the rough drip shield surface. There were substantial differences between the mechanisms of rivulet formation and movement on smooth and rough drip shield surfaces. Water collected in breaches was a function of the location of drip impact upstream from the target breach, i.e., impact breaches must be directly above or slightly to the side of the breaches in order for a substantial volume of water to collect in breaches. Splash droplets contributed a small portion of the water collected in breaches. Mass balances showed that evaporation from the drip shield was a large component of water loss. This was particularly manifested during low flow runs of the bounding flow rate tests where test duration was around five hours. 


\section{CONTENTS}

EXECUTIVE SUMMARY ...........................................................................................

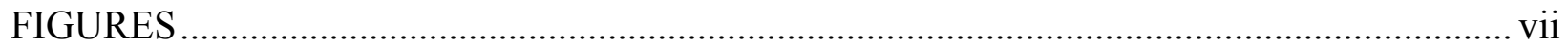

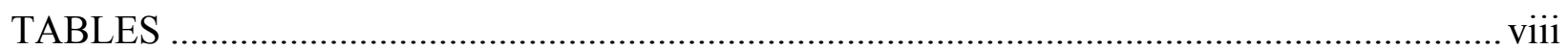

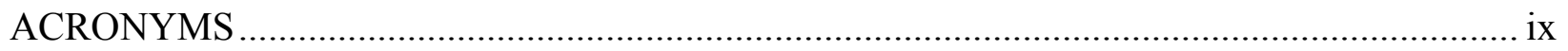

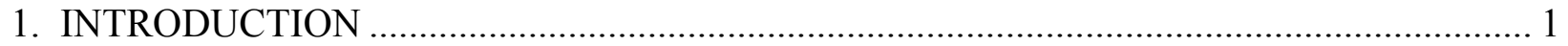

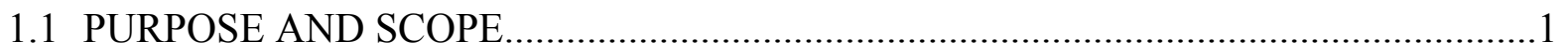

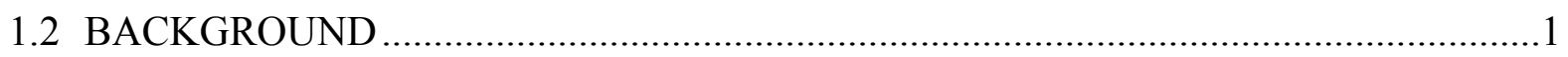

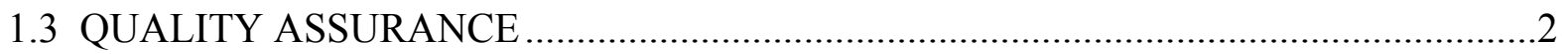

1.4 EBS BREACHED DRIP SHIELD TEST DESCRIPTION ............................................

2. TEST CONFIGURATION .................................................................................... 4

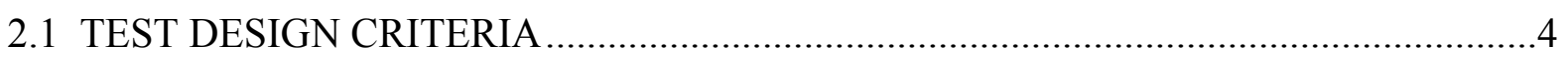

2.2 DRIP SHIELD MATERIALS AND SURFACE CONDITIONS ...................................

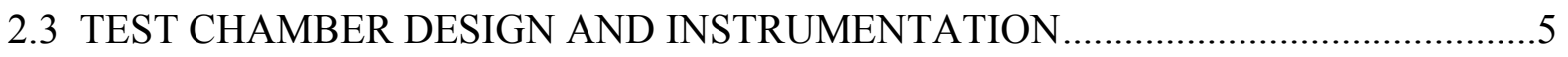

2.4 WATER INJECTION AND COLLECTION SYSTEMS .........................................5

2.5 SUPPORTING MEASUREMENTS .........................................................................

2.6 INSTRUMENT ACCURACY AND ERROR .......................................................... 7

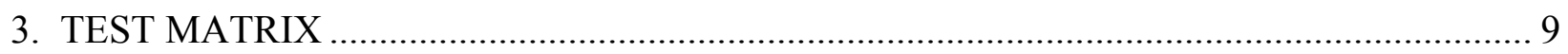

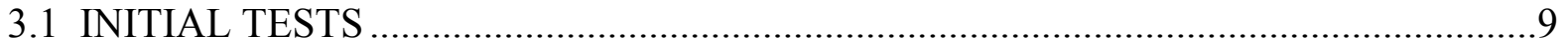

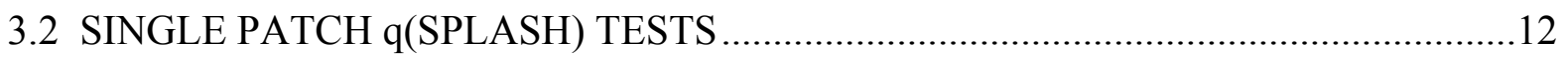

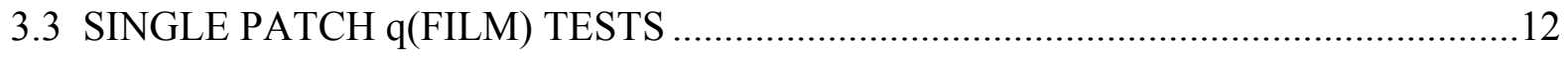

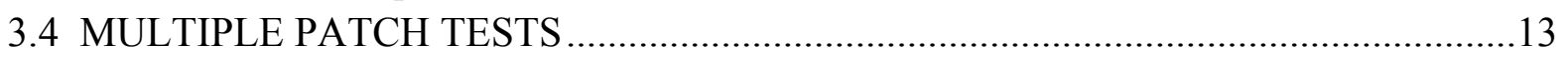

3.5 BOUNDING FLOW RATE TESTS ................................................................... 13

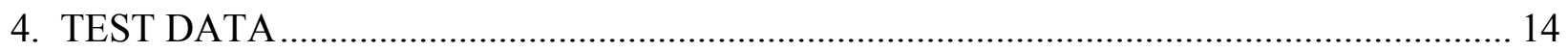

4.1 BWPDSE TEST DATA TRACKING NUMBERS ................................................... 14

4.2 BWPDSE TEST OBSERVATIONS AND DATA ANALYSIS ...................................15

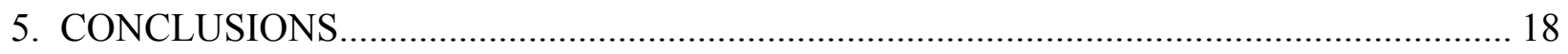

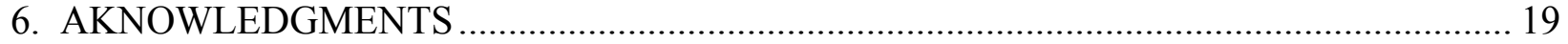

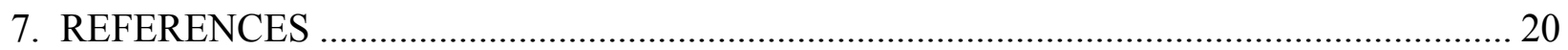

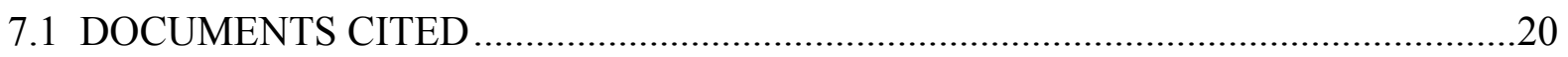

7.2 CODES, STANDARDS, REGULATIONS, AND PROCEDURES .............................21

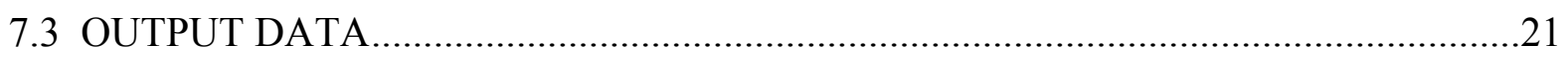

APPENDIX A: M\&TE Used in the BWPDSE .............................................................. A1

APPENDIX B: Collection Station Percentage Data ................................................................

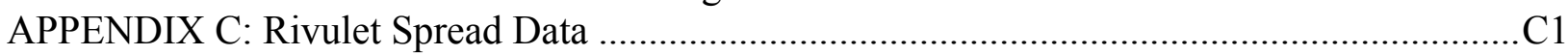

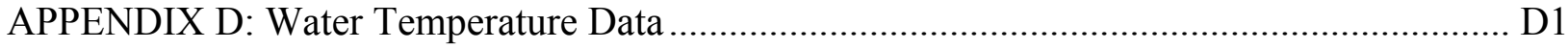




\section{FIGURES}

Page

Figure 1. Seepage Area Represented in the BWPDSE ................................................. F1

Figure 2. Drip Shield Design Dimensions and Patch Locations ......................................... F2

Figure 3. Stainless Steel Drip Shield Section …............................................................ F3

Figure 4. Polished Stainless Steel Drip Shield Surface (Splash Radius Test \#1 - 2 Drops)........ F4

Figure 5. Rough Drip Shield Surface with Collection Gutters ........................................... F5

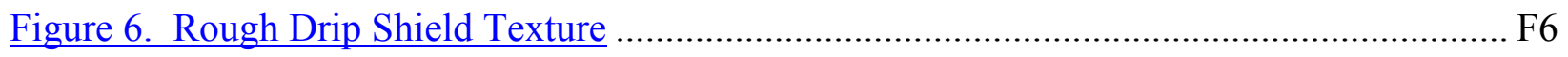

Figure 7. Test Chamber Environment Control and Instrumentation ..................................F7

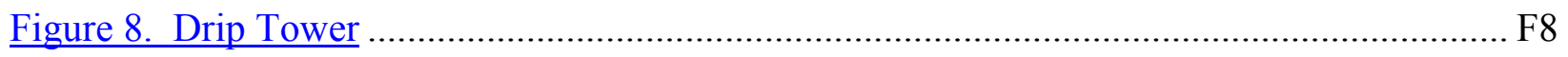

Figure 9. Collection System Gutters and Splash Shields.................................................. F9

Figure 10. Splash Radius Test \#1 Geometry on Smooth DS Surface................................... F10

Figure 11. Splash Radius Test \#2 Geometry on Smooth DS Surface................................... F11

Figure 12. Spread Factor Test \#1 Geometry on Smooth DS Surface .................................. F12

Figure 13. Spread Factor Test \#4 Geometry on Smooth DS Surface ................................... F13

Figure 14. Single Patch q(splash) Tests on Smooth DS Surface ......................................... F14

Figure 15. Single Patch $\mathrm{q}($ splash) Tests on Rough DS Surface ........................................... F15

Figure 16. Single Patch q (film) Tests on Smooth DS Surface ......................................... F16

Figure 17. Single Patch q(film) Tests on Rough DS Surface …........................................ F17

Figure 18. Multiple Patch Tests on Smooth and Rough DS Surfaces .................................. F18

Figure 19. Water Droplet Formation on the Smooth DS Surface......................................... F19

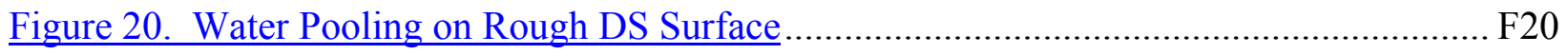




\section{TABLES}

\section{Page}

Table 1 EBS/Repository Design Parameters ……………................................................... T1

Table 2. BWPDSE Instrument Accuracy......................................................................

Table 3. Water Measurement Descriptions........................................................................ T2

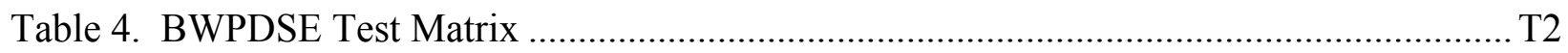

Table 5. Single Patch q(splash) Drip Location on Smooth DS Surface ..................................... T3

Table 6. Single Patch Drip Locations on Rough DS Surface ................................................... T3

Table 7. Single Patch q(film) Drip Locations on Smooth DS Surface .......................................T4

Table 8. Single Patch q(film) Drip Locations on Rough DS Surface ........................................ T5

Table 9. Multiple Patch Drip Locations for Smooth and Rough DS Surface Tests .................... T5

Table 10. Bounding Flow Rate Drip Locations for Smooth and Rough DS Surface Tests......... T5 


\section{ACRONYMS}

\begin{tabular}{|c|c|}
\hline $\begin{array}{l}\text { AMR } \\
\text { AP }\end{array}$ & $\begin{array}{l}\text { analysis model report } \\
\text { administrative procedure }\end{array}$ \\
\hline BWPDSE & Breached Waste Package and Drip Shield Experiments \\
\hline CRWMS & Civilian Radioactive Waste Management System \\
\hline $\begin{array}{l}\text { DOE } \\
\text { DI } \\
\text { DS } \\
\text { DTN }\end{array}$ & $\begin{array}{l}\text { Department of Energy } \\
\text { deionized } \\
\text { drip shield } \\
\text { data tracking number }\end{array}$ \\
\hline EBS & Engineered Barrier System \\
\hline $\begin{array}{l}\text { HLW } \\
\text { HMP }\end{array}$ & $\begin{array}{l}\text { high-level radioactive waste } \\
\text { humidity moisture probe }\end{array}$ \\
\hline M\&TE & measuring and test equipment \\
\hline $\begin{array}{l}\text { QA } \\
\text { QARD }\end{array}$ & $\begin{array}{l}\text { quality assurance } \\
\text { quality assurance requirements and description }\end{array}$ \\
\hline $\begin{array}{l}\text { RH } \\
\text { RTD }\end{array}$ & $\begin{array}{l}\text { relative humidity } \\
\text { resistance temperature device }\end{array}$ \\
\hline $\begin{array}{l}\text { TCO } \\
\text { TDMS }\end{array}$ & $\begin{array}{l}\text { YMP Test Coordination Office } \\
\text { Technical Data Management System }\end{array}$ \\
\hline WP & waste package \\
\hline
\end{tabular}




\section{INTENTIONALLY LEFT BLANK}




\section{INTRODUCTION}

\subsection{PURPOSE AND SCOPE}

Data collected from the Breached Waste Package and Drip Shield Experiments (BWPDSE) will be used to support the flux splitting model used in future revisions of the Analysis and Modeling Report (AMR) ANL-WIS-PA-000001, EBS Radionuclide Transport Abstraction (BSC 2001a). The purpose of the BWPDSE was to collect information on water flow paths and penetration through breaches on drip shield surfaces so that the flux splitting model can be both validated and made more complete with respect to water mass losses caused by splashing and evaporation.

The scope of this report is to document the BWPDSE by:

- Presenting test configurations and operating conditions

- Summarizing test data and providing data submittal tracking numbers

- Presenting test results

Both waste package (WP) and drip shield (DS) geometries are applicable to the flux splitting model and data generated in these tests will be used to describe seepage flux entering breaches through both DSs and WPs. A DS surface was used in these tests for simplification, and future testing to support the EBS radionuclide transport abstraction may include tests performed on WP surfaces if it is determined that such data are required.

\subsection{BACKGROUND}

The Engineered Barrier System (EBS) represents one system in the performance of the Yucca Mountain high-level radioactive waste (HLW) repository to isolate and prevent the transport of radionuclides from the site to the accessible environment. The primary transport mechanism for radionuclides is liquid water that may penetrate the EBS through breaches in the drip shields (DS) and waste packages (WP), which make up the components of the EBS. Water flux (represented by the letter "q") can enter the breaches via three different mechanisms: q(direct), a droplet falls directly through the patch (hole); q(film) liquid enters a breach by film or rivulet flow and; q(splash) liquid enters a breach as a result of liquid being pushed or lofted from a point of drip impact on the DS. A patch is a modeling term for a breach in the DS or WP. The flux splitting model (BSC 2001a) is conservatively based on the following assumptions:

1. Drip impact occurs without a loss of water mass from splattering and evaporation.

2. Dripping flux falls exactly at the crown of the DS. This limits the zone of impact to the horizontal surface across the DS crown and does not include the curved surface that would cause droplets to be deflected from the DS.

3. The flux passing through a DS patch is proportional to the ratio of the length of the penetration in the axial direction to the total axial length of the DS. In this assumption 
all fluid that drips and flows from the DS crown toward a penetration will be collected if the axial locations of the source and patch coincide (BSC 2001a).

The current model does not consider drop mass loss and splash radius following impact. The water droplets projected during impact, in reality, may land on the DS surface in another location or be completely thrown off of the DS. Because natural processes were in effect, these experiments represented effects not considered in the current models, which were not capable of incorporating the random processes inherent in water dripping onto both dry and wetted surfaces.

Test controls included the drip seepage rate, drip impact location, breach location, and breach size. General corrosion breaches were the only breaches considered in these tests and were based on the nodes or square patches of area $0.072144 \mathrm{~m}^{2}(0.2685963-\mathrm{m}$ square) from the WAPDEG Analysis of Waste Package and Drip Shield Degradation AMR (CRWMS M\&O 2000d). For testing purposes the patch size in the BWPDSE was rounded to $0.270-\mathrm{m}$ square in place of the patch size (0.2685963-m square) used in CRWMS M\&O 2000d. The drip shield length was constructed to 10 patch lengths, or $2.7 \mathrm{~m}$. In CRWMS M\&O 2000d, general corrosion patches did not appear on DSs before 10,000 years after placement, while manufacturing defects were predicted to appear after 1000 years.

Environmental conditions of interest in the BWPDSE were temperature and relative humidity $(\mathrm{RH})$. The test was performed in an enclosed insulated chamber where the $\mathrm{RH}$ was elevated above $80 \%$. It is important to note that these tests did not consider the effects of elevated temperature. It was not the intent to maintain test environment conditions, i.e., temperature and $\mathrm{RH}$, at the expected repository environment, but conditions were maintained to provide accurate water collection.

\subsection{QUALITY ASSURANCE}

This technical report was prepared in accordance with Administrative Procedure (AP)-3.11Q, Technical Reports. The technical work plan (TWP) that governed the work for the BWPDSE was TWP-MGR-MD-000015 Rev. 2, Technical Work Plan For: Engineered Barrier System Department Modeling and Testing FY 02 Work Activities (BSC 2002a), which was prepared in accordance with AP-2.27Q, Rev. 0, Quality Determination and Planning for Scientific, Engineering, and Regulatory Compliance Activities. Specific activities for the BWPDSE, including the writing of this report, are described in The Test Plan for: Atlas Breached Waste Package and Drip Shield Experiments, SITP-02-EBS-005 (BSC 2002b). This activity was subject to the Quality Assurance Requirements and Description (DOE 2003) requirements.

Other quality assurance features of this testing program include:

The Field Work Package (FWP)-EBS -02-001, Engineered Barrier Systems - Breached Waste Package and Drip Shield Testing (YMP 2002) which defines the execution plan, facility requirements, and health and safety guidelines of the BWPDSE. 
Details of the test configuration and records of the test execution were documented in scientific notebook numbers SN-M\&O-SCI-043-V1, SN-M\&O-SCI-043-V2, and SN-M\&O-SCI-043-V3, per AP-SIII.1Q, Scientific Notebooks (Howard 2002a, Howard 2002b, and Howard 2002c).

All instruments were calibrated in the Bechtel Nevada calibration laboratory and calibrations were documented in scientific notebook SN-M\&O-SCI-043-V1 (Howard 2002a, pp. 24-34) and SN-M\&O-043-V2 (Howard 2002b, pp. 223-240).

All data were entered manually into the scientific notebook, and then entered manually into MS Word documents for submittal to the TDMS. Data were reviewed and verified in accordance with AP-2.14Q prior to being submitted to the TDMS. Because the data were entered manually into the scientific notebook and then submitted to the TDMS via MS Word documents, the only AP-SV.1Q controls listed in the TWP that applied were those outlining personal computer security and a regular back up of test files. These controls were in place during the BWPDSE.

The only software utilized during the BWPDSE was MS Excel ' 97 for performing calculations and making data plots. All formulas, inputs and outputs are documented and this software is, therefore, exempt from the requirements of AP-SI.1Q per AP-3.11Q Sec. 5.1.3e.

During the BWPDSE, BSC QA conducted a surveillance (Krisha 2002) and found the testing to be programmatically compliant. No conditions adverse to quality were identified.

\subsection{EBS BREACHED DRIP SHIELD TEST DESCRIPTION}

The BWPDSE was conducted at the Department of Energy's National Nuclear Security Administration Nevada Support Facility in North Las Vegas, NV in the A-1 lowbay between May 2, 2002 and July 25, 2002. Testing was performed by dripping water on smooth and rough DS surfaces with cut square breaches $(0.270-\mathrm{m}$ square). Water was dripped on the DS surfaces

at nominal rates of $2 \mathrm{~m}^{3} / \mathrm{yr}(3.8 \mathrm{~g} / \mathrm{min}), 0.2 \mathrm{~m}^{3} / \mathrm{yr}(0.38 \mathrm{~g} / \mathrm{min})$, and $20 \mathrm{~m}^{3} / \mathrm{yr}(38 \mathrm{~g} / \mathrm{min})$ and allowed to collect in breaches or gutters placed on the DS boundaries. Following each test, the collected water was weighed at each station and a mass balance performed. Before tests were conducted, initial tests were performed to determine splash distances after drop impact and lateral rivulet spread from the line of impact. In some of the initial tests Blue \#1 food coloring was used in the drip water in order to make rivulets and splashes more visible on the drip shield surface. This practice was used sparingly during the initial tests and was not used during the formal testing because the food coloring stained the DS surface, making it necessary to thoroughly clean the DS surface before the next test run. The measurements taken during the initial tests were used to select drip impact locations for formal tests. All tests were performed at full-scale with respect to drop distance and DS curvature. 


\section{TEST CONFIGURATION}

\subsection{TEST DESIGN CRITERIA}

The following criteria applied to the test design:

1. DS breaches represent square general corrosion patches of area $0.072144 \mathrm{~m}^{2}(0.2685963$ by $0.2685963 \mathrm{~m}$ ), based on the AMR ANL-EBS-PA-000001 REV 00 ICN 01 WAPDEG Analysis of Waste Package and Drip Shield Degradation (CRWMS M\&O 2000d). Actual breaches used in the BWPDSE were machined (or cut) into the DS with dimensions of 0.270 by $0.270 \mathrm{~m}$.

2. The WP diameter $(1.564 \mathrm{~m})$ used in the BWPDSE represents those of the 21 PWR WP (CRWMS M\&O 2000b). The WP diameter is the WP footprint beneath the DS and represents the seepage area over the DS as illustrated in Figure 1.

3. The dripping distance was based on AMR design parameters, and thus drip velocity was represented on a full scale. The EBS/repository design parameters and values used in the BWPDSE, including reference to full DS dimensions, are listed in Table 1. Based on the values listed in Table 1, the distance from the top of the drift to the crown of the DS is $2.173 \mathrm{~m}$. Drip impact locations were positioned along lateral lines at the crown, $33^{\circ}$ along the DS curvature, and $16.5^{\circ}$ along the DS curvature (see Figure 1).

4. The nominal seepage drip rate was based on an average of the seepage flux rates expected in the repository (BSC 2001b). It is assumed that the DS will intercept all seepage flux entering the drift and for the purposes of these tests, seepage flux was represented as drips per unit time per unit area of DS above the waste package (WP) footprint. A nominal seepage rate of $2 \mathrm{~m}^{3} / \mathrm{yr}$ was used in the $\mathrm{q}$ (splash) and q(film) tests, while bounding rates of $0.2 \mathrm{~m}^{3} / \mathrm{yr}$ and $20 \mathrm{~m}^{3} / \mathrm{yr}$ were used during the bounding flow rate tests.

5. The condition of DS surfaces during the time they are not expected to fail (i.e., 1000 years for manufacturing defects and 10,000 years for general corrosion) was difficult to predict and replicate. Two different surfaces were tested: a standard machined stainless steel surface (the most conservative condition), and a rough surface that only approximated DS conditions expected to exist during DS failure. Both surface conditioning processes can be replicated.

6. The tests were conducted in an environment that would minimize evaporation, but not necessarily simulate repository conditions. This was achieved by conducting the tests in an insulated test chamber. During formal testing (not including all initial tests), the relative humidity $(\mathrm{RH})$ in the chamber was maintained above $80 \%$. The test chamber temperature was not directly controlled during testing, but was monitored. 


\subsection{DRIP SHIELD MATERIALS AND SURFACE CONDITIONS}

The DS was constructed of $1 / 4$ inch stainless steel and supported by an aluminum frame. For purposes of the BWPDSE, a full-scale section of DS was used in place of a complete DS. The DS section design was based on the full DS dimensions outlined in Table 1 . The DS was $2.7-\mathrm{m}$ long, $1.27-\mathrm{m}$ wide, and $1.62-\mathrm{m}$ high. A diagram of the drip shield with dimensions and patch (breach) locations is shown in Figure 2 and a picture of the DS and frame can be viewed in Figure 3 (Howard 2002a, p. 14 and p. 16). The smooth DS surface was formed by polishing the stock stainless steel with 60-grit sandpaper on a circular sander. A close-up photograph of the stainless steal DS surface is shown in Figure 4. The rough surface was formed by covering the stainless steel DS surface with a silica anti-slip coating (Howard 2002b, p. 70). A picture of the rough DS can be viewed in Figure 5 and a picture of the texture can be viewed in Figure 6.

\subsection{TEST CHAMBER DESIGN AND INSTRUMENTATION}

The test chamber was constructed from standard metal framing material and plywood. The chamber was insulated with R-5.7 rated insulation all sides and ceiling (Howard 2002c, p. 113). The test chamber dimensions were 3.66-m long, 2.74-m wide, and 2.44-m high (Figure 7). Water vapor was introduced into the chamber with the use of commercial humidifiers. Initially two humidifiers were used. A third humidifier was added before the $\mathrm{q}(\mathrm{film})$ tests (see Section 2.1.6) on the smooth DS for the purpose of increasing the $\mathrm{RH}$ at a faster rate and maintaining a higher $\mathrm{RH}$ in the chamber. During testing the $\mathrm{RH}$ was maintained at $80 \%$ or higher. A ventilation fan was used during testing to circulate air around the test chamber. Figure 7 shows a diagram of the test chamber with locations of humidifiers, the ventilation fan, and monitoring instrumentation. Test chamber instrumentation is listed with all testing M\&TE in Appendix A. The test chamber ceiling had three slots positioned along the length of the chamber ceiling to allow water drips to fall on the DS crown, the $16.5^{\circ}$ line, and the $33^{\circ}$ line. The slots were covered during testing with a 1.5-in styrofoam sheet, except for one of the many pre-cut access holes where the drip tower (see Section 2.4.1) was located. The test chamber environment data were collected before and after each test and recorded by hand on data collection sheets that were entered into the scientific notebook.

\subsection{WATER INJECTION AND COLLECTION SYSTEMS}

\subsubsection{Water Injection}

Water drips were introduced into the test environment by injecting water through an aquarium diffuser using a metering pump. The aquarium diffuser is a porous medium that allows water drops to collect slowly and fall after enough mass has collected to break the surface tension. A test performed to measure the droplet mass from an aquarium diffuser resulted in a mean mass of 0.141 grams (Howard 2002b, pp. 4-6). This method was used because it is similar to the processes that occur when water droplets collect and fall from the drift crown. The diffuser was mounted in a clear PVC tube (drip tower) and was adjusted to three different heights during the tests, which represent the drip distances between the DS surface at the crown, $16.5^{\circ}$, and $33^{\circ}$ and 
the corresponding position on the drift wall (see Figure 1). Figure 8 presents a photograph of the drip tower showing the three possible drip positions at 2.17-m (crown), 2.22-m $\left(16.5^{\circ}\right)$, and 2.31$\mathrm{m}\left(33^{\circ}\right)$ (Howard 2002a, p. 36). The drip tower was movable and was positioned over the designated impact location through the one of the three slots in the test chamber ceiling.

The input water rate was measured by weighing the initial and final water masses over the test time duration. Test duration times were measured with a stopwatch from the time the first drop impacted the drip shield to the time when the metering pump was shut off.

\subsubsection{Water Collection System}

Water that was dripped over the DS surface was collected at various stations that constituted water passing through breaches, water passing around breaches and running off the DS, water splashing over the edge of the DS boundary, and residual water that adhered to the DS surface. Water that ran into breaches was collected in pans that were mounted on the inside of the DS. Paper towels were placed inside the collection pans to prevent water from splashing out of the pan and to direct water into the pan from the breach inside edges. Water that passed by breaches and off the DS surface collected in gutters mounted to the front and back of the DS. Water that splashed off the front, back, and sides of the DS was caught and directed into the same collection gutters used to collect runoff. Residual water that remained on the DS surface was wiped up with pre-weighed lint-less paper towels. Diagrams of the gutters and splash shields mounted on the DS are shown in Figure 9. Six breaches and four gutters were used during the tests. The numbering system for the breaches is shown in Figure 4 and the numbering system for the gutters and splash shields is shown in Figure 9.

Water was collected from the gutters and splash shields in the same manner as it was collected from the DS surface. Water was wiped up with pre-weighed lint-less paper towels from gutters and splash shields, then weighed to determine the net moisture picked up by the towels. Water mass collected through breaches was determined by pre-weighing the collection pans then weighing them again following each test. The water masses collected at each station (i.e., breaches 1-6, gutters 1-4, and splash shields 1-4) were recorded by hand on data sheets then attached in the scientific notebook.

In the course of the testing it became evident that exposed towels could exchange moisture mass with the test chamber air for a net mass gain or loss. In order to provide data on the possible mass change for exposed towels, a reference towel was used in tests starting with the single patch tests for the rough DS surface. These data were applied only to the towels used to prevent splashing in the breach collection pans because the towels used to swab water from the DS, gutters, and splash shields were either weighed shortly before use or placed in pre-weighed sealed containers before and after use.

\subsection{SUPPORTING MEASUREMENTS}

Supporting measurements were those additional measurements that did not contribute directly to the collection of water, but provided additional data to add to the understanding of water pathways, in the form of rivulet flow and evaporation from the DS surface. These additional 
measurements include pan evaporation, water temperature, and rivulet spread. These measurements were not taken during every test, but enough measurements were taken to provide sufficient data to understand these processes.

\subsubsection{Pan Evaporation Measurements}

Evaporation in the test chamber was measured during tests with a $20.3-\mathrm{cm}$ by $20.3-\mathrm{cm}$ square plastic pan. The procedure used was to fill the pan with enough water to just cover the bottom then weigh the pan and water before and after the test. The pan was weighed without water to get a tare weight of 67.7 grams (Howard 2002a, p. 70). The evaporation pan data is not a direct representation of evaporation from the DS surface because of the differences between the exposed water surface area on the DS surface and the pan. The evaporation pan data is used as a general reference for the evaporation potential inherent under chamber conditions.

\subsubsection{Rivulet Spread Measurements}

Rivulet spread measurements were taken as a part of the initial tests on the smooth and rough DS surfaces to provide data for determining the impact locations for the formal testing. The practice of measuring the maximum and minimum rivulet spread was made a part of the data collection routine during the single patch $\mathrm{q}($ film) tests on the smooth DS surface. Measurements were performed with a commercial ruler or tape measure from the axial line of drip impact down the face of the DS. The convention for measurement direction was positive to the DS right and negative to the DS left when facing the DS front. Measurements were recorded to the nearest $0.5-\mathrm{cm}$.

\subsubsection{Water Temperature Measurements}

Water temperature measurements were taken during the final week of bounding flow rate tests on the rough DS. The four types of temperature measurements taken were input water, DS beaker water, DS surface water, and air temperature. Descriptions of these measurements are provided in Table 3. The primary objective of these measurements was to collect water temperature data to aid in the understanding of water evaporation from the DS surface.

\subsection{INSTRUMENT ACCURACY AND ERROR}

Instrument accuracies for the BWPDSE are listed in Table 2 and are stated in the initial entry of the scientific notebook SN-M\&O-SCI-043-V1 (Howard 2002a, p. 5). Instrument unique identifiers and their calibration status can be found in the scientific notebook and in Appendix A of this report.

There were two different analytical balance models used during the tests, each model having a different specification for accuracy. The model used to weigh input water was the same or less accurate than the balance used to weigh collection water. In this scenario, the mass balances test 
data (see Appendix B) are limited in accuracy by the less accurate model (or input mass). Because of this limitation, the accuracy for the less accurate model was used as the standard for water masses during the test and the mass balance data reported in Appendix B of this report has been truncated to reflect this. 


\section{TEST MATRIX}

The tests conducted during the BWPDSE on both smooth and rough DS surfaces include initial (or preliminary) splash radius (drip splash) and spread factor, single patch q(splash), single patch $\mathrm{q}($ film), and multiple patch tests. The test descriptions and geometries are presented in the following sections. A list of the tests conducted for the BWPDSE is provided in Table 4.

\subsection{INITIAL TESTS}

\subsubsection{Initial Splash Radius Tests on the Smooth DS Surface}

The objective of the splash radius tests was to determine the splash distance from the point of drip impact. The two measurements of interest from the splash radius tests are: 1) the radius of the bulk or cluster of splash droplets that accumulate around the impact point, and 2) the farthest drip splash thrown from the impact point which is referred to as the fringe. The data in these tests were used to set the impact point distance from the patch (breach) center in the single patch $\mathrm{q}$ (splash) tests. Two splash radius tests were conducted on the smooth DS surface.

\subsubsection{Splash Radius Test \#1 - Smooth DS}

Splash radius test \#1 was performed by dripping deionized (DI) water on the DS crown at a location of $27 \mathrm{~cm}$ from the DS centerline as illustrated in Figure 10 (The DS centerline is centered on Patch 6). The drops were counted and the drip radius measurements were taken at regular intervals during the test. Drip measurements were taken along three axes at $00^{\circ}, 90^{\circ}$, and $180^{\circ}$ (see Figure 10). The distance from the drip injection point to the impact point was $2.17 \mathrm{~m}$. The patch located at the crown was covered to prevent water from entering. Relative humidity, temperature, and barometric pressure were measured inside and outside of the test chamber before and after the test.

\subsubsection{Splash Radius Test \#2 - Smooth DS}

Splash radius test \#2 was performed by dripping DI water with Blue \#1 food coloring on the DS centerline at $33^{\circ}$ from the crown as illustrated in Figure 11. The drops were counted and the drip radius measurements were taken at regular intervals during the test. Measurements were reported to the nearest whole $\mathrm{cm}$. Drip measurements were taken along three axes at 0,180 , and 270 degrees (see Figure 11). The distance from the drip injection point to the impact point was $2.31 \mathrm{~m}$. Patch \#2 was covered to prevent water from entering. Relative humidity, temperature, and barometric pressure were measured inside and outside of the test chamber before and after the test. 


\subsubsection{Initial Spread Factor Tests on the Smooth DS Surface}

The objective of the spread factor tests was to determine the lateral rivulet spread distance from the drip impact point. The data collected in these tests was used to set the impact point distance from the patch (breach) center in the single patch q(film) tests.

\subsubsection{Spread Factor Test \#1 - Smooth DS}

Spread factor test \#1 was performed by dripping DI water on the DS Crown at a location of 27 $\mathrm{cm}$ from the DS centerline as illustrated in Figure 12. The drops were counted and rivulet spread measurements were taken at regular intervals during the test. The pump was stopped to take measurements. The distances from the line of impact on the DS surface to the rivulets were measured at $33^{\circ}$, the transition between the vertical and curved sections, and at the top of the patches on the vertical section. The distance from the drip injection point to the impact point was $2.17 \mathrm{~m}$. The breaches located at the crown (breach 6) and at the vertical center position (breach 2) were covered to prevent water from entering (see Figure 12). Relative humidity, temperature, and barometric pressure were measured inside and outside of the test chamber before and after the test.

\subsubsection{Spread Factor Test \#2 - Smooth DS}

Spread factor test \#2 was performed by dripping DI water with Blue \#1 food coloring on the DS crown at a location of $27 \mathrm{~cm}$ from the DS centerline. The test geometry and method used to measure rivulet spread was the same as spread factor test \#1 (see Figure 12).

\subsubsection{Spread Factor Test \#3 - Smooth DS}

Spread factor test \#3 was performed by dripping DI water with Blue \#1 food coloring on the DS crown at a location of $27 \mathrm{~cm}$ from the DS centerline. The drops were allowed to accumulate on the DS surface and form rivulets without stopping the pump. After a period of time when several rivulets had formed, the pump was shut off and the rivulet spread was measured for each rivulet that remained on the DS. The measurement convention was positive for rivulets to the right of the drip impact center and negative for rivulets to the left. The distances from the line of impact to the rivulets were measured at $33^{\circ}$, the transition between the vertical and curved sections, and at the top of the patches on the vertical section. The distance from the drip injection point to the impact point was $2.17 \mathrm{~m}$. The breaches located at the crown (breach 6) and at the vertical center position (breach 2) were covered to prevent water from entering. The geometry was the same as that used in spread factor test \#1 (see Figure 12). Relative humidity, temperature, and barometric pressure were measured inside and outside of the test chamber before and after the test.

\subsubsection{Spread Factor Test \#4 - Smooth DS}

Spread Factor Test \#4 was performed by dripping DI water with blue \#1 food coloring on the DS centerline at $33^{\circ}$ as illustrated in Figure 13. The method used to collect measurements was the 
same as spread factor test \#3. The distance from the drip injection point to the impact point was $2.31 \mathrm{~m}$. The breaches located at the crown (breach 6) and at the vertical center position (breach 2) were covered to prevent water from entering (see Figure 13). Relative humidity, temperature, and barometric pressure were measured inside and outside of the test chamber before and after the test.

\subsubsection{Initial Splash Radius Tests on the Rough DS Surface}

Splash radius tests were conducted on the rough DS surface with the same method as those conducted on the smooth DS surface, however, the test matrix was modified to provide more data that represents characteristics of the rough surface. Three test drip impact locations were tested on the rough DS surface at the crown, $16.5^{\circ}$, and $33^{\circ}$. Because the rough DS surface reacted differently than the smooth surface, three replicate tests were performed at the crown drip impact location. The data in these tests were used to set the impact point distance from the patch (breach) center in the single patch q(splash) tests.

\subsubsection{Splash Radius Tests \#1 - \#3 - Rough DS}

Splash radius tests \#1 through \#3 on the rough DS surface were performed in the same manner as splash radius test $\# 1$ conducted on the smooth DS surface. DI water was dripped on the DS crown at a location of $27 \mathrm{~cm}$ left of the DS centerline as illustrated. The methods and geometry were the same as splash radius test \#1 on the smooth DS surface, with the exception that the drip impact location was located to the left of DS center instead of the right as illustrated in Figure 9.

\subsubsection{Splash Radius Tests \#4 and \#5 - Rough DS}

Splash radius tests \#4 and \#5 on the rough DS surface were performed by dripping DI water at a location of $27 \mathrm{~cm}$ from the DS centerline at $33^{\circ}$ and $16.5^{\circ}$, respectively. The method was the same as that used in splash radius tests \#1 - \#3. The geometry for tests \#4 and \#5 differ with drip impact points located at $33^{\circ}$ (2.31-m drop distance) and $16.5^{\circ}$ (2.22-m drop distance).

\subsubsection{Initial Spread Factor Tests on the Rough DS Surface}

Spread factor tests were conducted on the rough DS surface with the same method as those conducted on the smooth DS surface. Three drip impact locations were tested on the rough DS surface at the crown, $16.5^{\circ}$, and $33^{\circ}$. Spread factor tests \#1, \#2, and \#3 on the rough DS surface were performed by dripping DI water at a location of $27 \mathrm{~cm}$ left of the DS centerline at $16.5^{\circ}$, $33^{\circ}$, and the crown, respectively. The drops were allowed to accumulate on the DS surface and form rivulets without stopping the pump. After a period of time when several rivulets had formed, the pump was shut off and the rivulet spread was measured for each rivulet that remained on the DS. The measurement convention was positive for rivulets to the right of the drip impact center and negative for rivulets to the left. The distances from the line of impact to the rivulets were measured at $33^{\circ}$, the transition between the vertical and curved sections, and at the top of the patches on the vertical section. 


\subsection{SINGLE PATCH q(SPLASH) TESTS}

The objective of the single patch $\mathrm{q}$ (splash) tests was to collect splash droplets only (excluding rivulets flow) that entered target breaches 4,5 , and 6 while water was dripped onto the DS section at a impact point within the bulk radius of splash determined in the splash radius tests. The single patch q(splash) tests were performed by dripping DI water on the DS at a locations determined during the initial splash radius tests for a period long enough to allow a sufficient collection of water in the breaches of interest. Breach 2 was covered to prevent water entry (see Figures 14 and 15). Relative humidity, temperature, and barometric pressure were measured inside and outside of the test chamber before and after the tests.

\subsubsection{Single Patch q(splash)Tests on the Smooth DS Surface}

The drip impact locations for the single patch $\mathrm{q}$ (splash) tests conducted on the smooth DS surface are listed in Table 5 and test geometry is illustrated in Figure 14.

\subsubsection{Single Patch q(splash) Tests on the Rough DS Surface}

The drip impact locations for the single patch $\mathrm{q}$ (splash) tests conducted on the rough DS surface are listed in Table 6 and test geometry is illustrated in Figure 15. It was evident during q(splash) tests on the smooth surface that the splash area patterns resulting from some drip locations did not meet the test objective to allow only splashes (excluding rivulet flow) to enter target breaches 4, 5, and 6 (see Section 3.2). For this reason, the number of drip locations was reduced for the single patch $\mathrm{q}$ (splash) tests on the rough DS based on observation and test results from the $\mathrm{q}$ (splash) tests on the smooth DS.

\subsection{SINGLE PATCH q(FILM) TESTS}

The objective of the Single Patch q(film) tests was to collect rivulet flows that entered patches 2, 4 , and 5 while water was dripped onto the DS section at an impact point within the rivulet spread range determined in the spread factor tests. The single patch $\mathrm{q}(\mathrm{film})$ tests were performed by dripping DI water on the DS at locations determined during the initial spread factor tests for a period long enough to allow a sufficient collection of water in the patches of interest. Breaches 1,3 , and 6 were covered to prevent water entry during the smooth DS tests (see Figure 16). No breaches were covered during tests performed on the rough DS surface because the collection of water in the additional breaches did not interfere with target breach collection and additional splash data could be obtained in breach 6 (see Figure 17). Relative humidity, temperature, and barometric pressure were measured inside and outside of the test chamber before and after the test. The single patch $\mathrm{q}($ film) tests were also referred to as rivulet flow tests.

\subsubsection{Single Patch q(film) Tests on the Smooth DS Surface}

The drip impact locations for the single patch q(film) tests conducted on the smooth DS surface are listed in Table 7 and test geometry is illustrated in Figure 16. 


\subsubsection{Single Patch q(film) Tests on the Rough DS Surface}

The drip impact locations for the single patch $\mathrm{q}($ film) tests conducted on the rough DS surface are listed in Table 8 and test geometry is illustrated in Figure 17.

\subsection{MULTIPLE PATCH TESTS}

The objective of the multiple patch tests was to collect both splash and rivulet flows that entered all affected patches while water was dripped onto the DS section at regular impact points along the DS axis. Multiple patch tests on the smooth and rough DS surfaces were performed by dripping DI water on the DS at locations shown listed in Table 9 and shown in Figure 18. The drip impact locations in Table 9 and Figure 18 apply to tests on the smooth and rough DS surfaces.

\subsection{BOUNDING FLOW RATE TESTS}

The objective of these tests was to provide data for the extreme drift seepage conditions to compare with test data performed at the nominal $2.0 \mathrm{~m}^{3} / \mathrm{yr}$ rate during the single and multiple patch tests. The bounding flow rate tests were carried out at seepage rates of $0.2 \mathrm{~m}^{3} / \mathrm{yr}$ and 20 $\mathrm{m}^{3} / \mathrm{yr}$. The bounding flow rate tests on the smooth and rough DS surfaces were performed by dripping DI water on the DS at locations listed in Table 10, which represent nine of the drip impact locations used in the multiple patch tests (see Figure 18). The low flow rate $\left(0.2 \mathrm{~m}^{3} / \mathrm{yr}-\right.$ nominal) tests were performed for approximately five hours for each location, while the high flow rate $\left(20 \mathrm{~m}^{3} / \mathrm{yr}-\right.$ nominal $)$ were performed for approximately 10 minutes. The drip impact locations in Table 10 apply to tests on the smooth and rough DS surfaces. 


\section{TEST DATA}

\subsection{BWPDSE TEST DATA TRACKING NUMBERS}

Data submittals outlined in this section represent data generated during the BWPDSE and logged in the scientific notebooks (Howard 2002a and Howard 2002b). Test data tracking numbers (DTNs) and titles for the BWPDSE are listed below:

Atlas Breached Waste Package and Drip Shield Experiments: Initial Tests for Rough Drip Shield Surface, DTN: MO0207EBSATBWP.021

Period: $6 / 26 / 2002$ to $6 / 28 / 2002$

Atlas Breached Waste Package and Drip Shield Experiments: Initial Tests for Smooth Drip Shield Surface, DTN: MO0207EBSATBWP.022

Period: $5 / 13 / 2002$ to $5 / 15 / 2002$

Atlas Breached Waste Package and Drip Shield Experiments: Single Patch q(splash) and q(film) Tests on the Smooth Drip Shield Surface, DTN: MO0207EBSATBWP.023

Period: $5 / 16 / 2002$ to $6 / 1 / 2002$

Atlas Breached Waste Package and Drip Shield Experiments: Multiple Patch Tests for Smooth Drip Shield Surface, DTN: MO0207EBSATBWP.024

Period: $6 / 3 / 2002$ to $6 / 14 / 2002$

Atlas Breached Waste Package and Drip Shield Experiments: Bounding Flow Rate Tests on the Smooth Drip Shield Surface, DTN: MO0207EBSATBWP.025

Period: $6 / 14 / 2002$ to $6 / 24 / 2002$

Atlas Breached Waste Package and Drip Shield Experiments: Single Patch q(splash) and q(film)

Tests on the Rough Drip Shield Surface, DTN: MO0208EBSATBWP.026

Period: $7 / 11 / 2002$ to $7 / 16 / 2002$

Atlas Breached Waste Package and Drip Shield Experiments: Multiple Patch Tests on the Rough Drip Shield Surface, DTN: MO0208EBSATBWP.027

Period: $6 / 28 / 2002$ to $7 / 25 / 2002$

Atlas Breached Waste Package and Drip Shield Experiments: Bounding Flow Rate Tests on the Rough Drip Shield Surface, DTN: MO0208EBSATBWP.028

Period: $7 / 16 / 2002$ 


\subsection{BWPDSE TEST OBSERVATIONS AND DATA ANALYSIS}

\subsubsection{Initial Test Observations}

The purpose of this section is to summarize the observations of the initial tests performed on the smooth and rough DS surfaces. Observations were particularly made with respect to droplet formation and rivulet spread in the splash radius and spread factor tests. Droplet formation for the smooth and rough DS surfaces was documented in photos for some of the initial tests. (Howard 2002a and Howard 2002b).

\subsubsection{Observations during Initial Tests Performed on the Smooth DS Surface}

An example of water droplet formation is presented in a photograph from splash radius test \#1 in Figure 19. Beads formed and increased in size around the center of impact with each successive drop. After a time, the beads closest to the downhill curvature would reach a critical mass and roll down the face of the drip shield in the form of a rivulet. Droplet splashing was observed on the crown as well as the curved drip shield surface within the tested seepage area (above the $33^{\circ}$ line). No film flow was observed during tests on the smooth DS surface.

The maximum lateral splash radius observed in splash radius test \#1 was $72.5 \mathrm{~cm}$ after 60 drops. As a comparison, the longest lateral splash distance observed in spread factor test \#2 was 71.0 $\mathrm{cm}$ after 478 drops. To determine the distance to be used in the q(splash) tests, a value of 70.0 $\mathrm{cm}$ was multiplied by 0.25 for a final distance of $17.5 \mathrm{~cm}$, which falls within the cluster values observed in the splash test observations after sufficient mass had been deposited on the DS surface to allow regular splashing from impact on water droplets.

When water was dripped at the crown, the rivulet flow area spreads out in a delta formation, meaning maximum spread was located on the vertical section of the DS and the minimum spread was located at the point of impact. The delta formation spread was less for drip impact locations on the $33^{\circ}$ line. Spread distances, distance from the patch/breach center, used during the single patch $\mathrm{q}($ film) tests for breaches 2, 4, and 5 were based on spread distances measured during impact on the crown. The spread distances used for the single patch tests on the smooth DS surface were: breach 2, $15 \mathrm{~cm}$ from patch center; breach 4, $8.0 \mathrm{~cm}$ from patch center; and breach $5,4.0 \mathrm{~cm}$ from patch center.

\subsubsection{Observations during Initial Tests Performed on the Rough DS Surface}

On the rough DS surface water beads formed from splashing would lead to the formation of small pools with constant dripping. A large pool would form around the drip impact center and spread. A photograph showing water pooling around the impact center is provided in Figure 20. Rivulet flow began much later on the rough DS surface after the pools grew large enough to reach the DS slope, causing the pools to drain down the DS face. As observed on the smooth drip shield surface, droplet splashing occurred on the curved section within the tested seepage area (above the $33^{\circ}$ line). Film flow was not observed during the tests on the rough DS surface. 
The maximum lateral $\left(0^{\circ}\right.$ or right) bulk splash radius was observed in splash test $\# 1$ at $48.0 \mathrm{~cm}$ after 203 drops. The distance used in the $\mathrm{q}$ (splash) tests was determined by multiplying a value of $40.0 \mathrm{~cm}$ by 0.5 for a final distance of $20 \mathrm{~cm}$. This final distance falls within the cluster values observed in the splash test observations after sufficient mass had been deposited on the DS surface to allow regular splashing from impact on water droplets. The splash radius on the rough DS was less than on the smooth DS surface. The outer splashes on the fringe tended to be smaller and less frequent than the fringe splashes on the smooth DS surface.

The spread factor distances used in the $\mathrm{q}$ (film) flow tests were designed to allow either all or a portion of the rivulets formed up gradient to enter at breaches 2,4 , and 5 . The rivulet spread distances from the drip centerline were much less on the rough DS surface than on the smooth DS. In many cases, rivulets flowed straight down the face of the drip shield with little lateral deviation. In addition, established flow paths were maintained, meaning there was a continuous flow of water in some rivulets. The impact points for the rough DS tests were set at $13.5 \mathrm{~cm}$ from the patch centers, or along the edge, for Patches 2, 3, and 4.

\subsubsection{Test Data Analysis}

Data from the single patch tests, multiple patch tests, and bounding flow rate tests were analyzed by performing a mass balance. As a part of the analysis the percentages were determined for water collected in each station as a function of the total input. Seepage rate, pan evaporation, and reference towel data were included as references. These data are listed in Appendix B with the test chamber environment conditions. A complete representation of the data is found in the TDMS submittals (see Section 4.1). The values listed in Appendix B have been truncated to the number of significant figures representing the instrument error of the weighing balance (see Section 2.6). Losses determined in the mass balances ranged from around $7 \%$ to the mid $50 \%$ range. The highest losses were observed during the low flow runs of the bounding flow rate tests. Most of the losses ranged from around $10 \%$ to $30 \%$. Losses in the mass balance can be attributed mainly to evaporation of water droplets from the DS surface and other collection surfaces (gutters and splash shields) with some loss attributed to the limitation of the collection procedure, i.e., collecting water by swabbing the DS and collection surfaces. In general, more losses were observed on the rough DS surface over the smooth DS surface. The water collection process, i.e., swabbing water from the DS surface, may have had a greater impact on experimental error than the weighing process. The greater losses observed on the rough DS surface can most likely be attributed to the added difficulty of swabbing water from the roughened texture.

\subsubsection{Rivulet Spread Data}

The rivulet spread from the impact centerline was measured for a number of tests (see Section 2.5.2). These data are listed in Appendix C. Rivulet spread ranged from 0 to around $45 \mathrm{~cm}$ from the drip impact line. In general the smooth DS surface produced a wider spread over the rough DS surface. In many cases on the rough DS, surface rivulet flow would start in a few main rivulets then maintain flow through those rivulets through the duration of the test. The smooth DS surface produced several variable flow paths during the single test. 


\subsubsection{Water Temperature Data}

Water temperature data were collected for the input water and the DS surface water during the last week of testing (see Section 2.5.3). These data are listed in Appendix D. The primary objective of these data was to aid in the understanding of evaporation from the DS surface. The DS surface water temperatures matched the air temperature (within tenths of $a^{\circ} \mathrm{C}$ ) and the DS beaker and input waters were generally lower than the air and DS surface water temperatures. 


\section{CONCLUSIONS}

The purpose of the BWPDSE was to provide data to support the validation and further development of the flux splitting model used in the EBS Radionuclide Transport Abstraction (BSC 2001a). The data gathered in these tests represent the possible flow paths that affect the entry of water through corrosion breaches on both smooth and rough DS surfaces. These data were limited by the instrument error information provided in Section 2.6. Uncertainties in the data were based primarily on limitations of the collection process, which was performed by swabbing up water from the DS surfaces, gutters, and splash shields. The differences in the water mass collection and seepage input (see Appendix B) can be attributed primarily to evaporation from the DS and other collection surfaces. The evaporation process from beaded water droplets on flat surfaces was much greater than the measured pan evaporation. It was evident from the bounding flow rate data that evaporation losses were greater when the test time was lengthened. Evaporation processes played a substantial role in water losses during the BWPDSE and should be investigated further. The following items summarize the conclusions of the BWPDSE:

- The primary flow mechanism on both smooth and rough surfaces was rivulet flow, not film flow.

- Lateral rivulet spread distances were, in general, wider on the smooth DS surface than on the rough DS surface.

- There were substantial differences between the mechanisms of rivulet formation and movement on smooth and rough DS surfaces.

- Water collected in breaches was a function of the location of drip impact upstream from the target breach, i.e., impact location must be directly above or slightly to the side of the breach in order for a substantial volume of water to collect in breach.

- Splash droplets contribute a small portion of the water collected in breaches.

- Mass balances showed that evaporation from the DS was a large component of water loss. This was particularly manifested during low flow runs of the bounding flow rate tests where test duration was around 5 hours.

- Mass balances were affected to some degree by the water collection process. This was manifested in the losses observed between tests conducted on the smooth and rough DS surfaces. 


\section{AKNOWLEDGMENTS}

The Breached Waste Package and Drip Shield Experiments were performed with help from the Engineered Barrier Systems test team. Cliff Howard was the responsible manager. Norm Kramer managed procurements, data submittals, and quality issues. Hemendra Kalia coordinated use of the North Las Vegas facility and construction support. Roy Johnston managed the day-to-day construction and test operations. John Del Mar performed many of tests and provided data analysis support. Neva Mason provided reference and technical reviews. Sandra Dalvit Dunn provided procedural guidance and technical reviews. Linda Croom assisted in the assembly and review of the document. Fred Homuth and Troy Williams of the TCO provided technical advice and instrumentation. 


\section{REFERENCES}

\subsection{DOCUMENTS CITED}

BSC (Bechtel SAIC Company) 2001a. EBS Radionuclide Transport Abstraction. ANL-WIS-PA000001 REV 00 ICN 03. Las Vegas, Nevada: CRWMS M\&O. ACC: MOL.20010806.0076.

BSC 2001b. FYO1 Supplemental Science and Performance Analyses, Volume 2: Performance Analyses. TDR-MGR-PA-000001 REV 00. Las Vegas, Nevada: CRWMS M\&O. ACC: MOL.20010724.0110.

BSC 2002a. Technical Work Plan For: Engineered Barrier System Department Modeling and Testing FY 02 Work Activities. Technical Work Plan. TWP-MGR-MD-000015 REV 02, Las Vegas, Nevada: BSC. ACC: MOL.20021014.0209.

BSC 2002b. Test Plan for: Atlas Breached Waste Package and Drip Shield Experiments. SITP-02-EBS-005 REV 00. Las Vegas, Nevada: CRWMS M\&O. ACC: MOL.20020314.0181.

CRWMS M\&O 1999. Request for Repository Subsurface Design Information to Support TSPASR. Input Transmittal PA-SSR-99218.Ta. Las Vegas, Nevada: CRWMS M\&O. ACC: MOL.19990901.0312.

CRWMS M\&O 2000a. Design Analysis for the Ex-Container Components. ANL-XCS-ME000001 REV 00. Las Vegas, Nevada: CRWMS M\&O. ACC: MOL. 20000525.0374.

CRWMS M\&O 2000b. Design Analysis for UCF Waste Packages. ANL-UDC_MD-000001 REV 00. Las Vegas, Nevada: CRWMS M\&O. ACC: MOL.20000526.0336.

CRWMS M\&O 2000c. Invert Configuration and Drip Shield Interface. TDR-EDS-ST-000001 REV 00. Las Vegas, Nevada: CRWMS M\&O. ACC: MOL.20000505.0232.

CRWMS M\&O 2000d. WAPDEG Analysis of Waste Package and Drip Shield Degradation. ANL-EBS-PA-000001 REV 00 ICN 01. Las Vegas, Nevada: CRWMS M\&O. ACC: MOL.20001208.0063.

DOE (U.S. Department of Energy) 2003. Quality Assurance Requirements and Description. DOE/RW-0333P, Rev. 13. Washington, D.C.: U.S. Department of Energy, Office of Civilian Radioactive Waste Management. ACC: DOC.20030422.0003.

Howard, C.L. 2002a. Breached Waste Package Test and Drip Shield Experiments. Scientific Notebook SN-M\&O-SCI-043-V1. ACC: MOL. 20021219.0022.

Howard, C.L. 2002b. Breached Waste Package Test and Drip Shield Experiments. Scientific Notebook SN-M\&O-SCI-043-V2. ACC: MOL. 20021219.0023.

Howard, C.L. 2002c. Breached Waste Package Test and Drip Shield Experiments. Scientific Notebook SN-M\&O-SCI-043-V3. ACC: MOL. 20021219.0024. 
Krisha, D.T. 2002. Bechtel SAIC Company, LLC (BSC) Quality Assurance (QA) Surveillance Report BSCQA-02-S-34 for Breached Waste Package Testing. Letter from D.T. Krisha (BSC) to T.W. Doering (BSC), July 3, 2002, RFH:ml-0702023204, with enclosure. ACC: MOL.20020815.0550.

YMP (Yucca Mountain Site Characterization Project) 2002. Engineered Barrier SystemsBreached Waste Package and Drip Shield Testing. Field Work Package FWP-EBS-02-001, Rev. 0. Las Vegas, Nevada: Yucca Mountain Site Characterization Office. ACC: MOL.20020409.0014.

\subsection{CODES, STANDARDS, REGULATIONS, AND PROCEDURES}

AP-2.20Q, Rev. 1, ICN 1. Self-Assessments. Washington, D.C.: U.S. Department of Energy, Office of Civilian Radioactive Waste Management. ACC: DOC.20030422.0005.

AP-2.27Q, Rev. 0, ICN 0. Planning for Scientific Activities. Washington, D.C.: U.S. Department of Energy, Office of Civilian Radioactive Waste Management. ACC: MOL.20021014.0209.

AP-3.11Q, Rev. 3, ICN 4. Technical Reports. Washington, D.C.: U.S. Department of Energy, Office of Civilian Radioactive Waste Management. ACC: DOC.20030331.0002.

AP-12.1Q, Rev. 0, ICN 2. Control of Measuring and Test Equipment and Calibration Standards. Washington, D.C.: U.S. Department of Energy, Office of Civilian Radioactive Waste Management. ACC: MOL.20020207.0111

AP-SI.1Q, Rev. 5, ICN 0. Software Management. Washington, D.C.: U.S. Department of Energy, Office of Civilian Radioactive Waste Management. ACC: DOC.20030422.0012.

AP-SIII.1Q, Rev. 2. Scientific Notebooks. Washington, D.C.: U.S. Department of Energy, Office of Civilian Radioactive Waste Management. ACC: MOL.20010905.0138

AP-SIII.7Q, Rev. 0, ICN 1. Scientific Investigation Laboratory and Field Testing. Washington, D.C.: U.S. Department of Energy, Office of Civilian Radioactive Waste Management. ACC: MOL.20010917.0189.

AP-SV.1Q, Rev. 0, ICN 3. Control of the Electronic Management of Information. Washington, D.C.: U.S. Department of Energy, Office of Civilian Radioactive Waste Management. ACC: MOL.20020917.0133

\subsection{OUTPUT DATA}

MO0207EBSATBWP.021. Atlas Breached Waste Package and Drip Shield Experiments: Initial Tests for Rough Drip Shield Surface. Submittal date: July 31, 2002. 
MO0207EBSATBWP.022. Atlas Breached Waste Package and Drip Shield Experiments: Initial Tests for Smooth Drip Shield Surface. Submittal data: July 31, 2002

MO0207EBSATBWP.023. Atlas Breached Waste Package and Drip Shield Experiments: Single Patch q(splash) and q(film) Tests on the Smooth Drip Shield Surface. Submittal data: July 31, 2002.

MO0207EBSATBWP.024. Atlas Breached Waste Package and Drip Shield Experiments: Multiple Patch Tests for Smooth Drip Shield Surface. Submittal data: July 31, 2002.

MO0207EBSATBWP.025. Atlas Breached Waste Package and Drip Shield Experiments: Bounding Flow Rate Tests on the Smooth Drip Shield Surface. Submittal data: July 31, 2002.

MO0208EBSATBWP.026. Atlas Breached Waste Package and Drip Shield Experiments: Single Patch q(splash) and q(film) Tests on the Rough Drip Shield Surface. Submittal data: August 13, 2002.

MO0208EBSATBWP.027. Atlas Breached Waste Package and Drip Shield Experiments: Multiple Patch Tests on the Rough Drip Shield Surface. Submittal data: August 13, 2002.

MO0208EBSATBWP.028. Atlas Breached Waste Package and Drip Shield Experiments: Bounding Flow Rate Tests on the Rough Drip Shield Surface. Submittal data: August 13, 2002. 


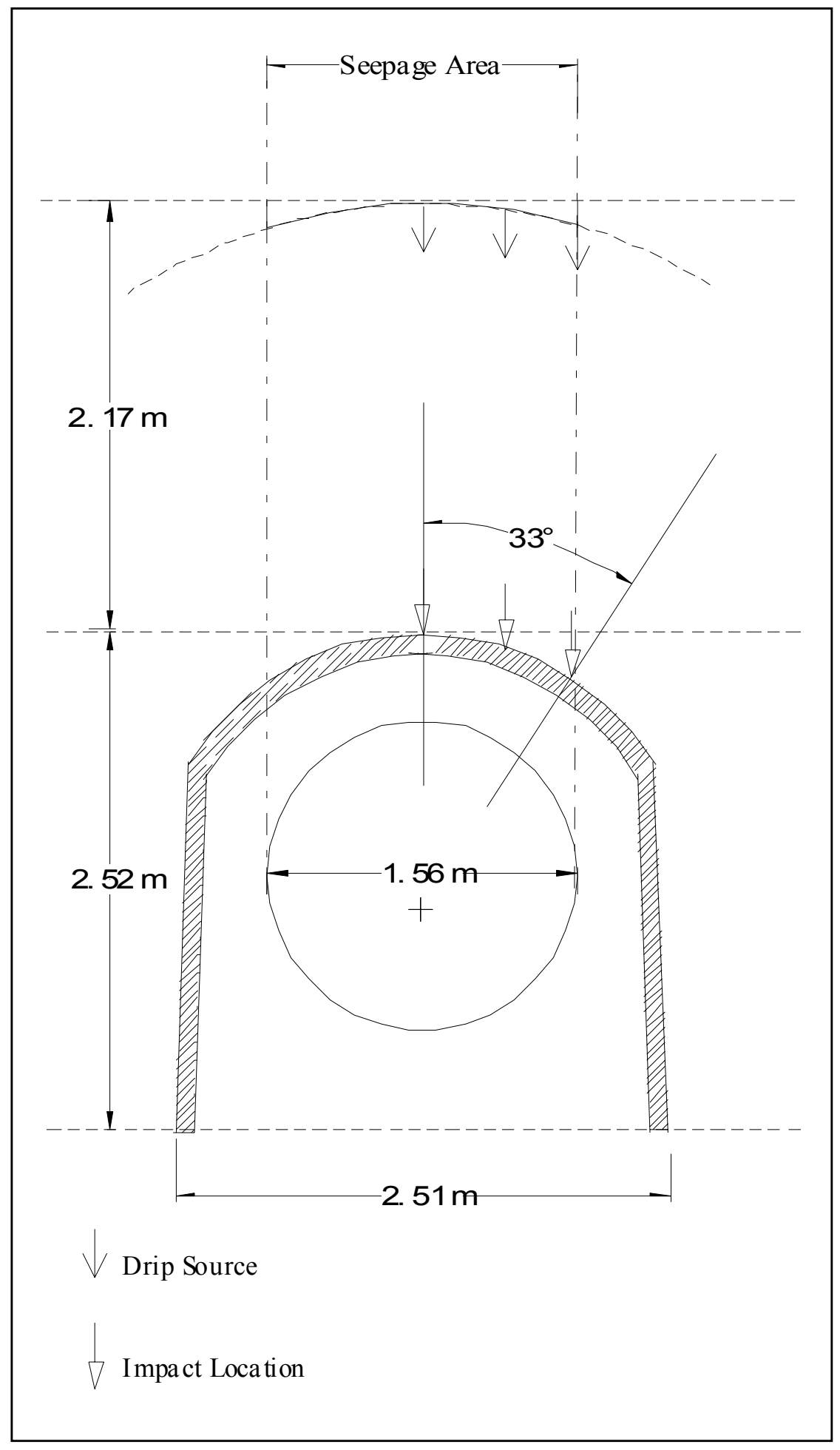

Source: Howard 2002c, p. 111.

Figure 1. Seepage Area Represented in the BWPDSE 


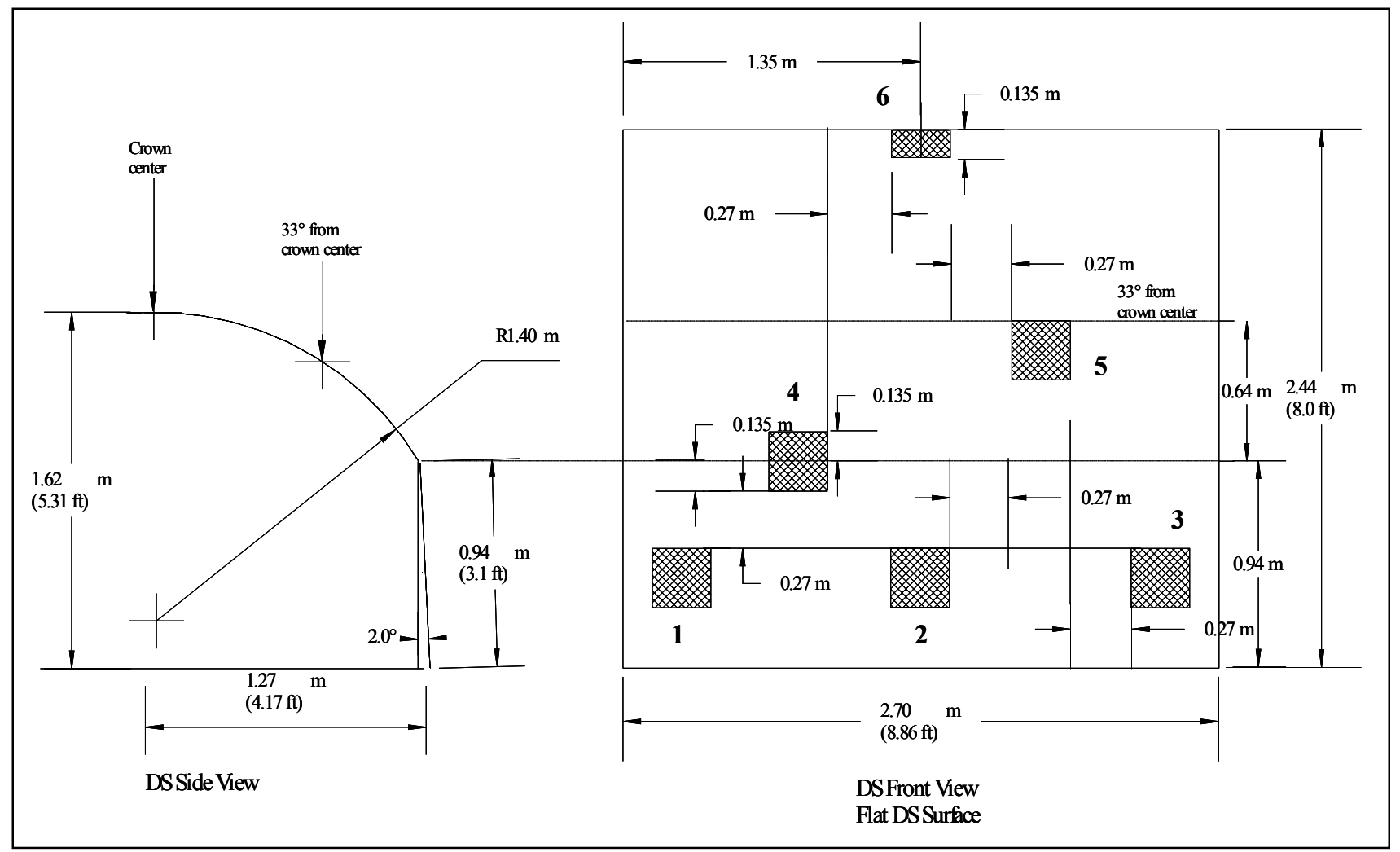

Source: Howard 2002a, p. 14.

Figure 2. BWPDSE Drip Shield Dimensions and Patch Locations 


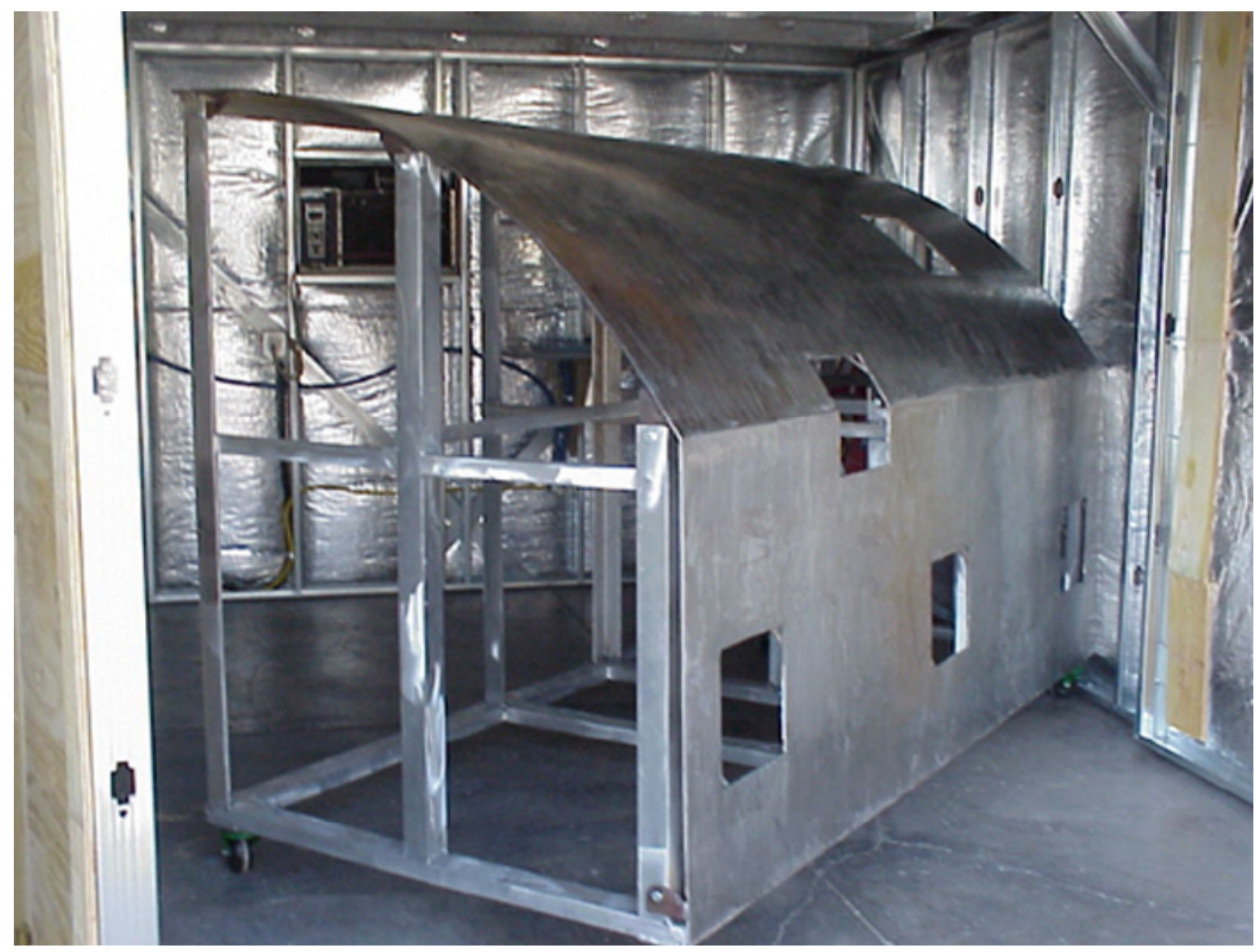

Source: Howard 2002a, p. 16.

Figure 3. Stainless Steel Drip Shield Section 


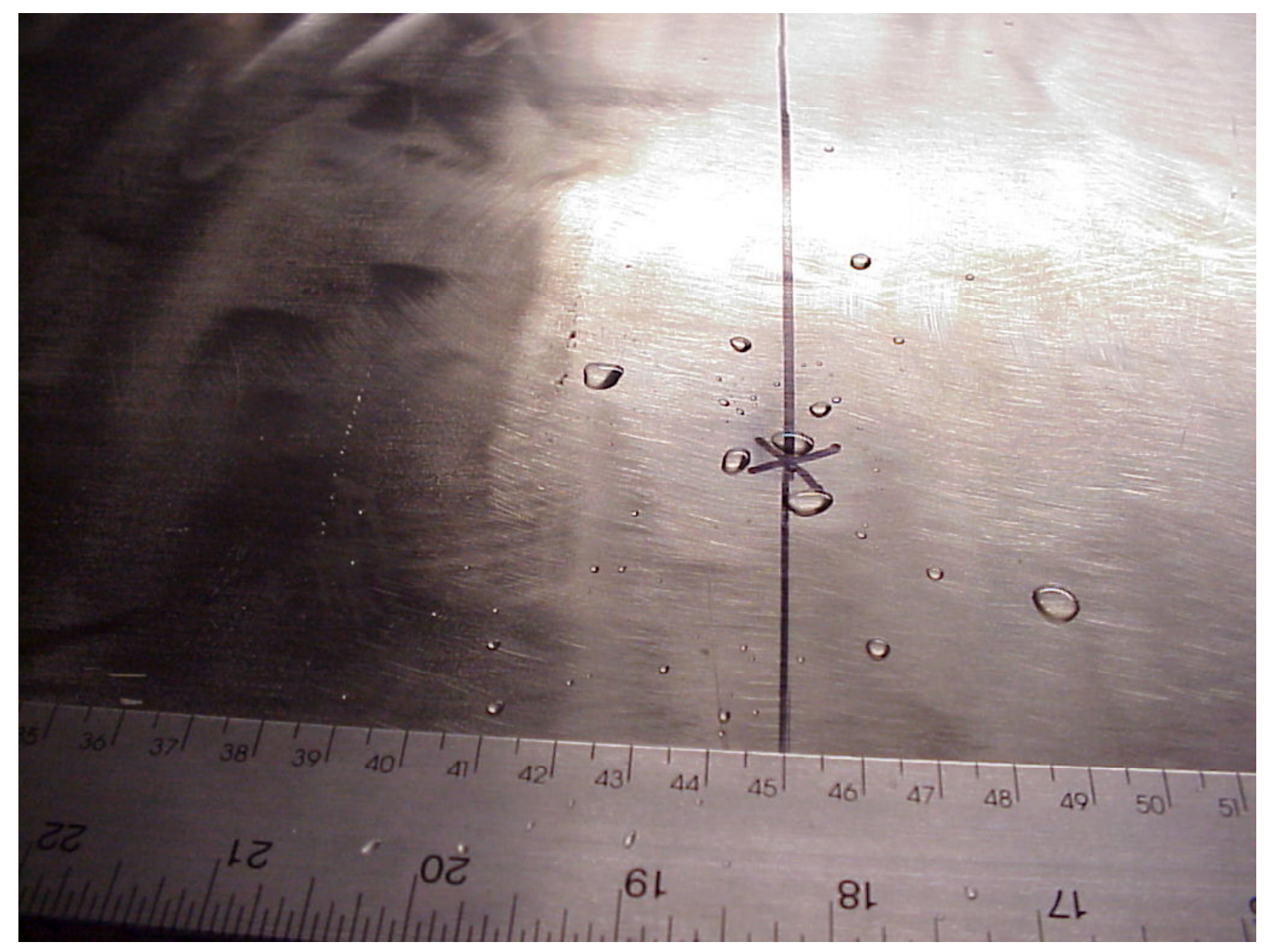

Source: Howard 2002a, p. 42.

Figure 4. Polished Stainless Steel Drip Shield Surface (Splash Radius Test \#1 - 2 Drops) 


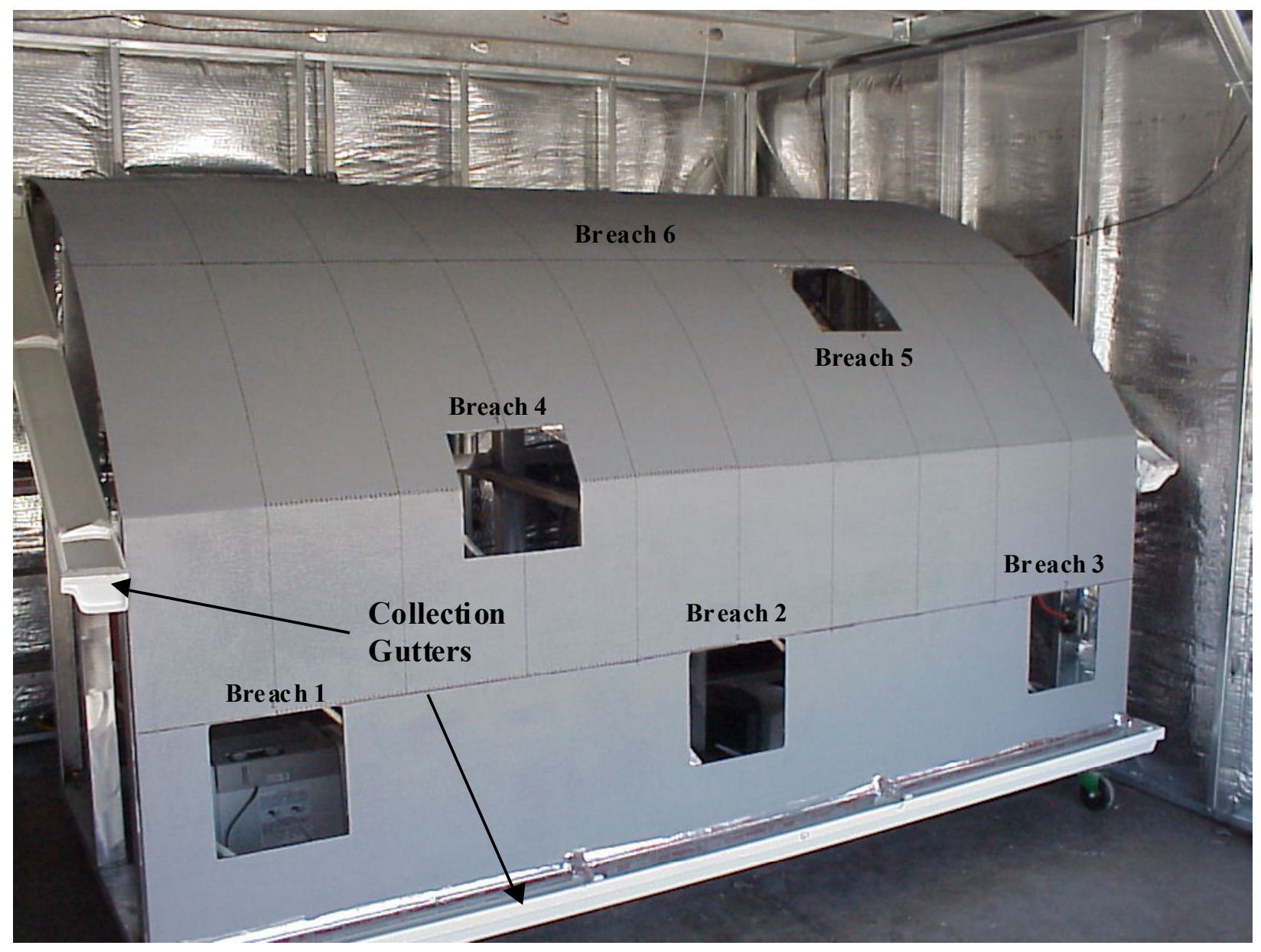

Source: Howard 2002c, p. 112.

Figure 5. Rough Drip Shield Surface with Collection Gutters 


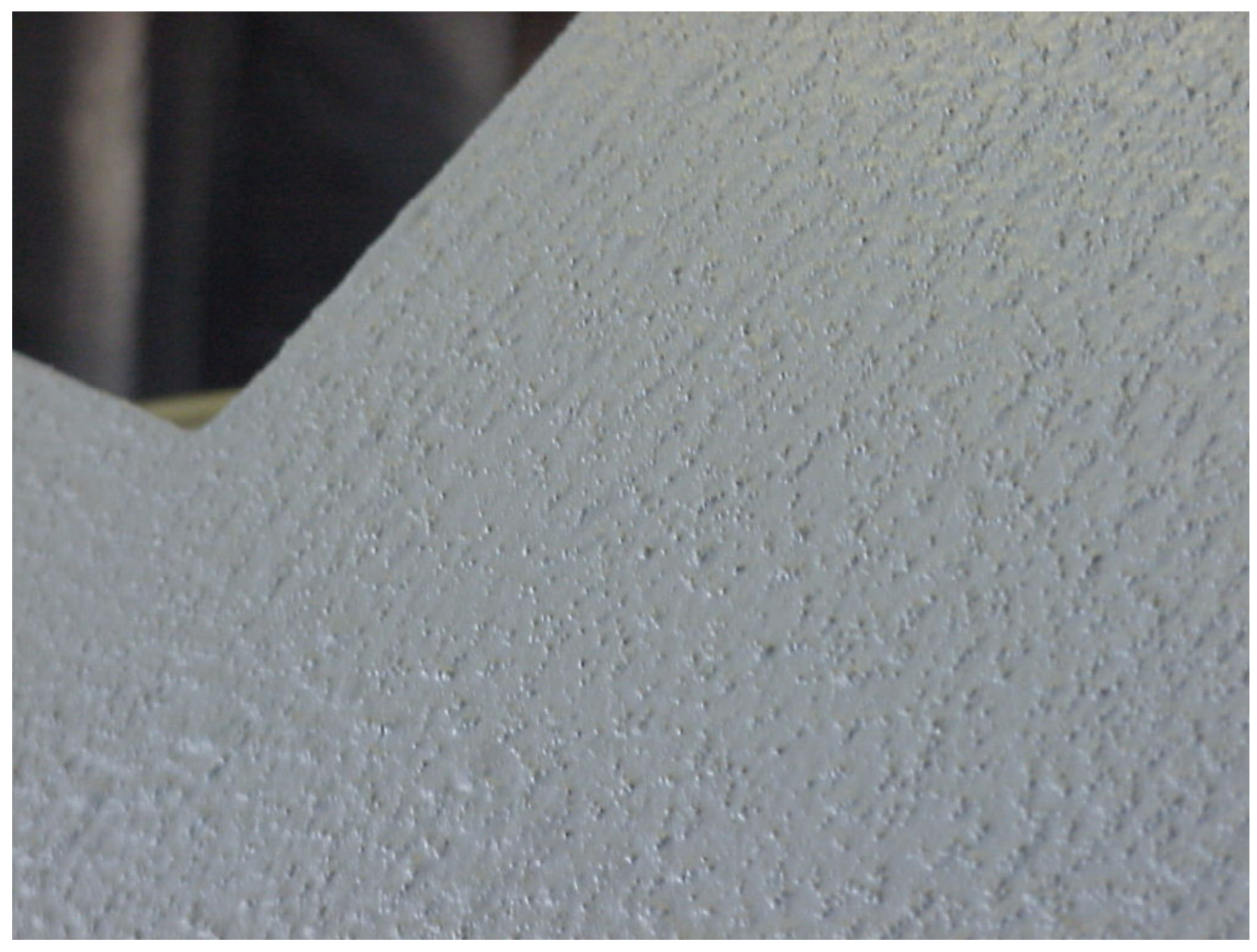

Source: Howard 2002b, p. 70.

Figure 6. Rough Drip Shield Texture 


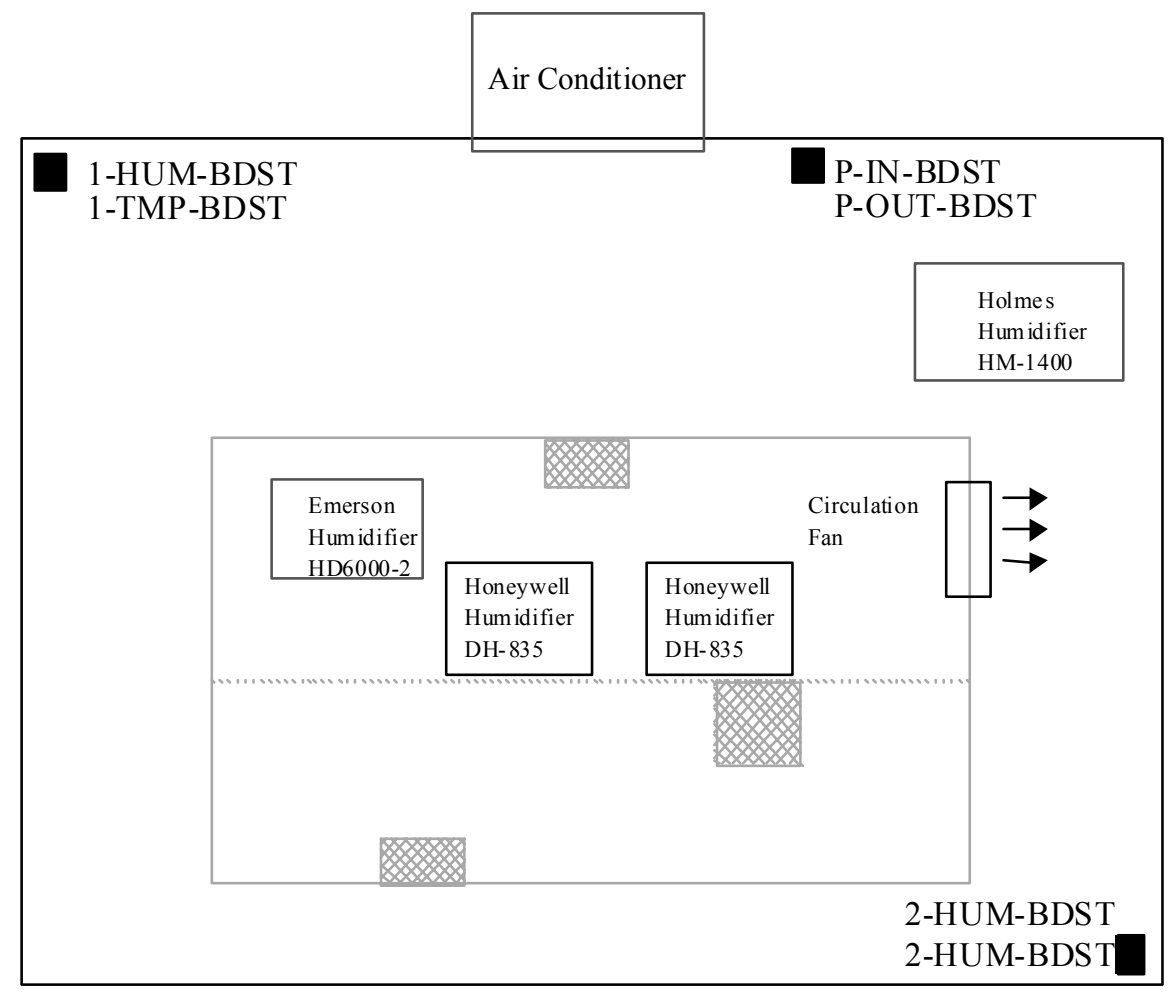

Drip Shield and Test Chamber (Plan View)

\section{Sensor Labels}

HUM-BDST: Relative Humidity

TMP-BDST: Temperature

P-IN-BDST: Inside Chamber Pressure

P-OUT-BDST: Outside Chamber Pressure

BDST (Breached Drip Shield Test)

Source: Howard 2002a, p. 108

Figure 7. Test Chamber Environment Control and Instrumentation 


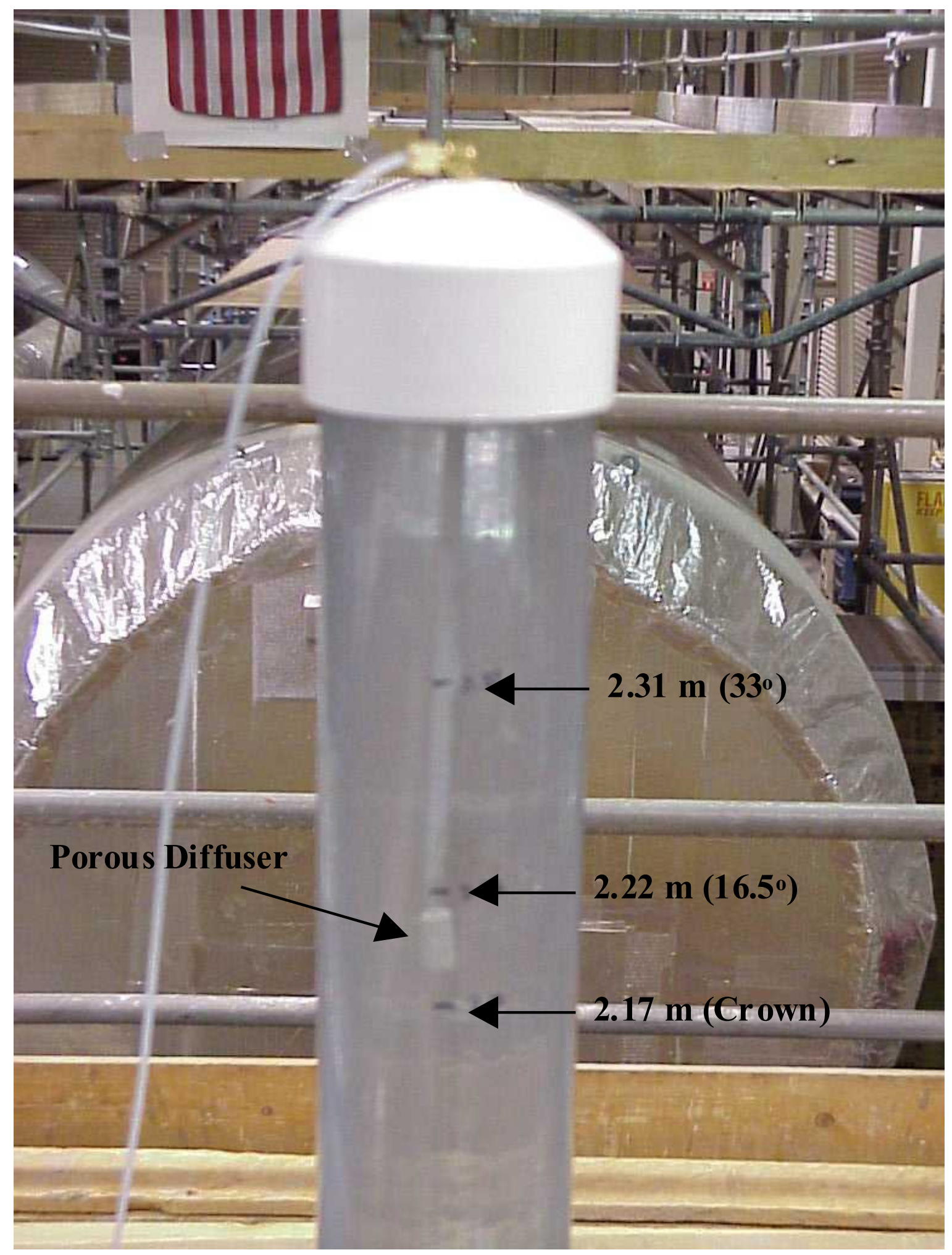

Source: Howard 2002a, p. 22.

Figure 8. Drip Tower 


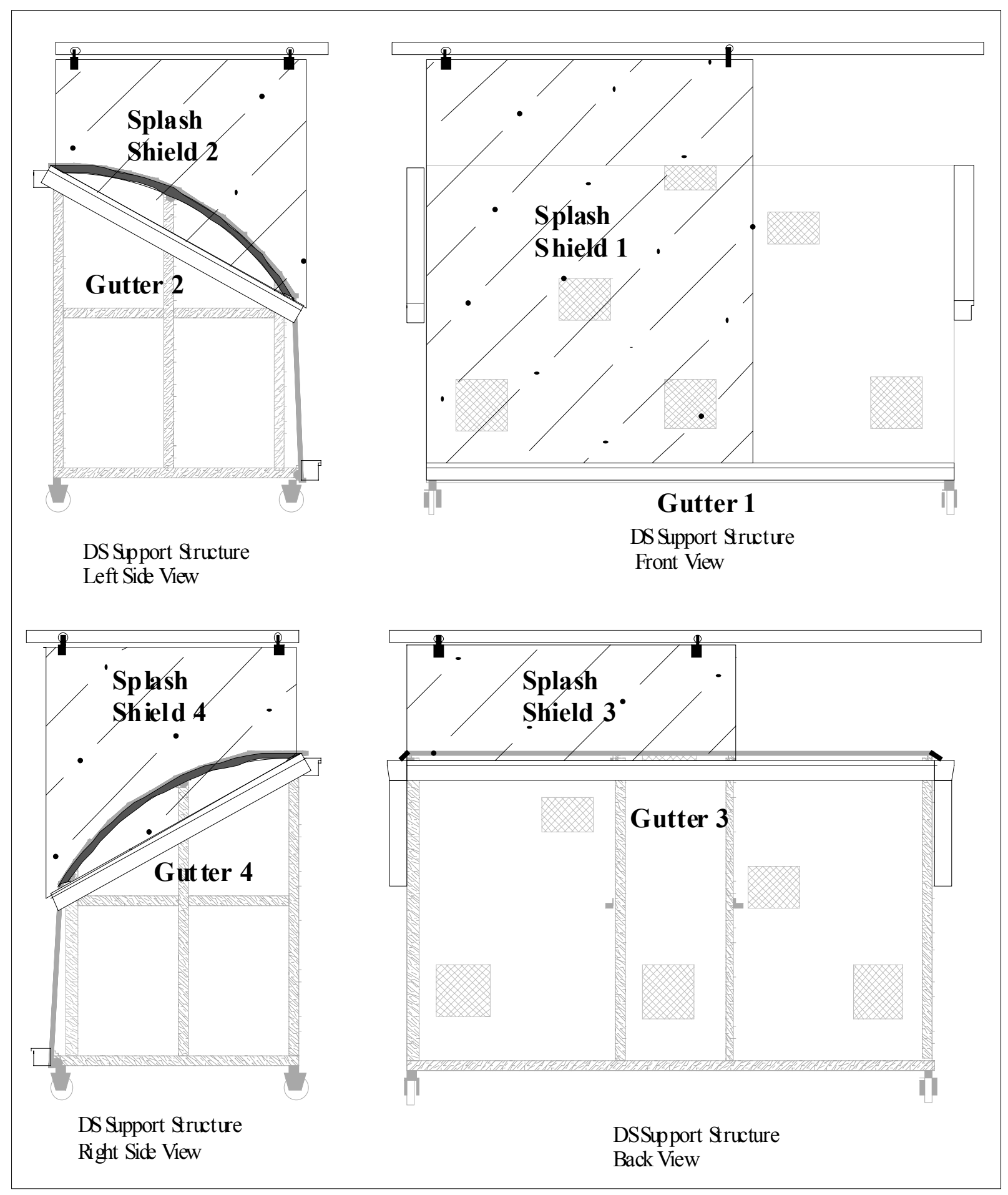

Source: Howard 2002a, pp. 18-19.

Figure 9. Collection System Gutters and Splash Shields 


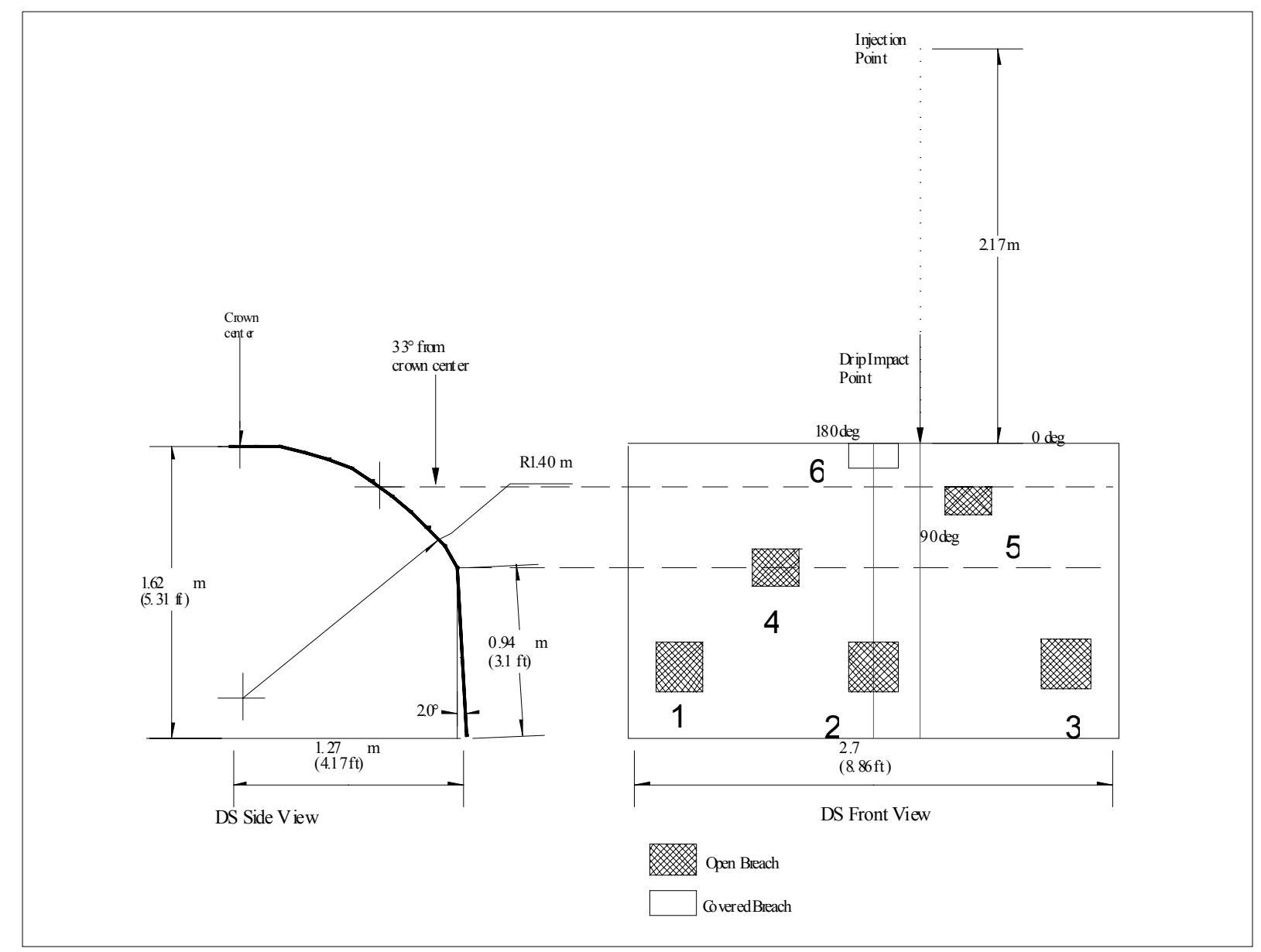

Source: Howard 2002a, p. 40.

Figure 10. Splash Radius Test \#1 Geometry on Smooth DS Surface 


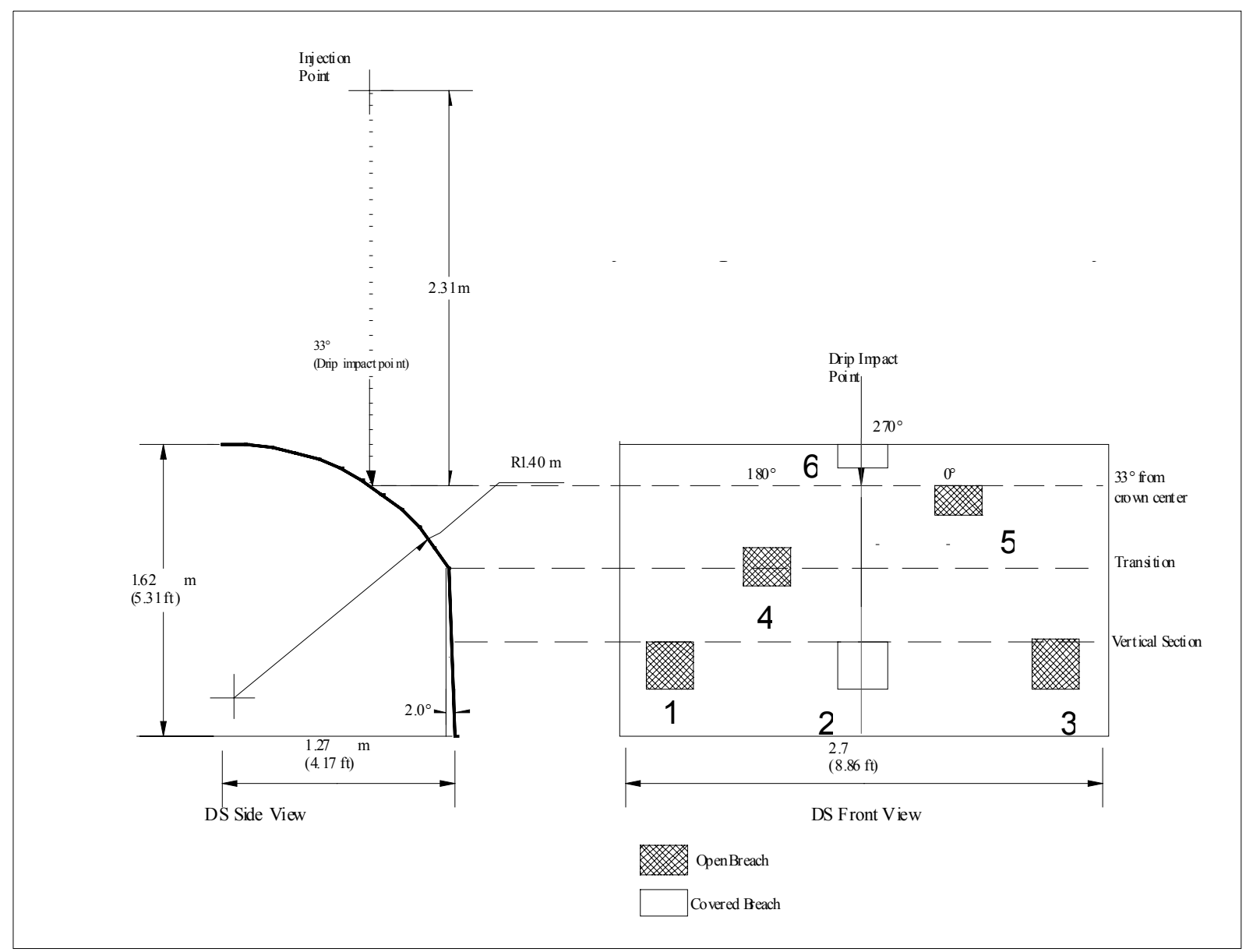

Source: Howard 2002a, p. 58.

Figure 11. Splash Radius Test \#2 Geometry on Smooth DS Surface 


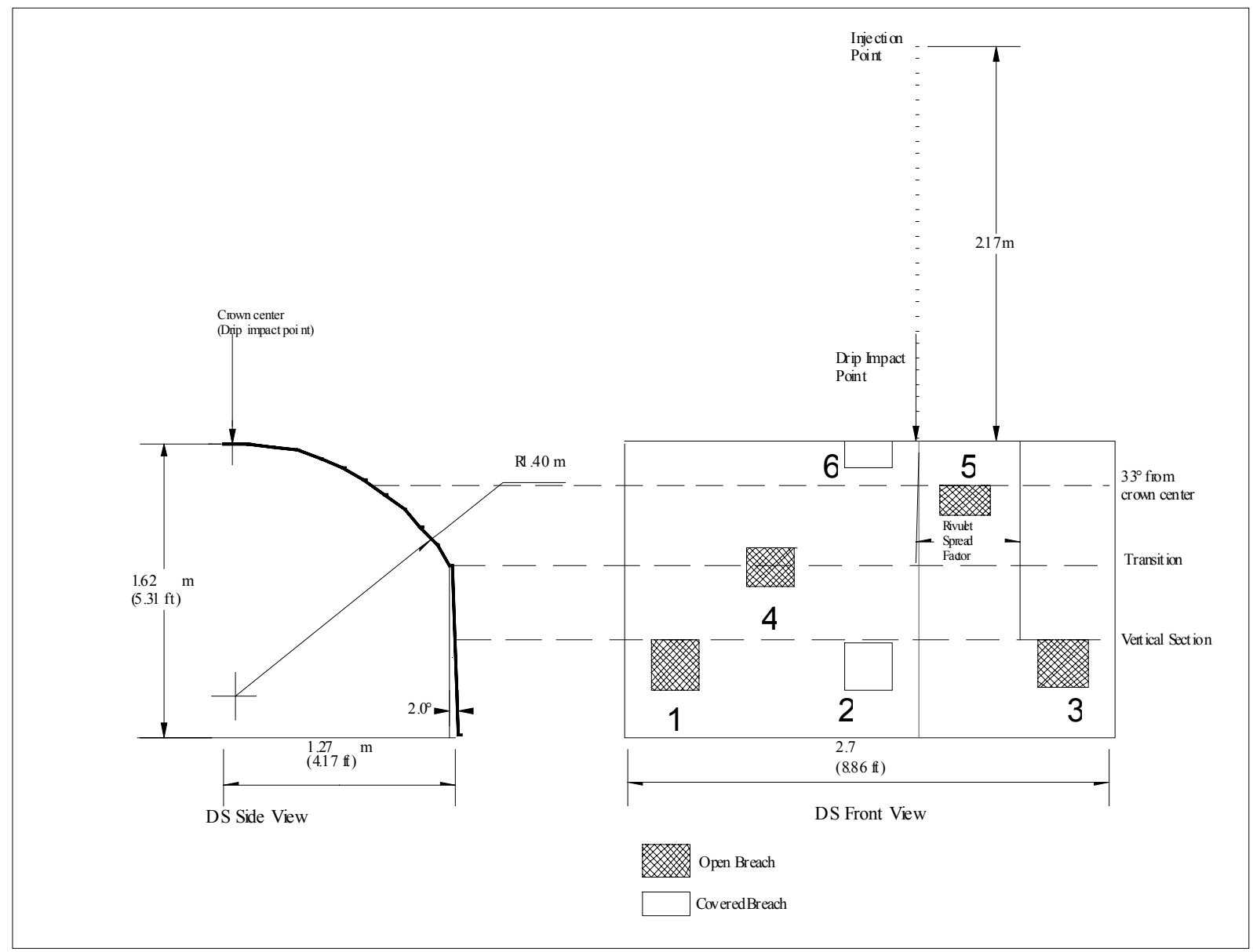

Source: Howard 2002a, p. 46.

Figure 12. Spread Factor Test \#1 Geometry on Smooth DS Surface 


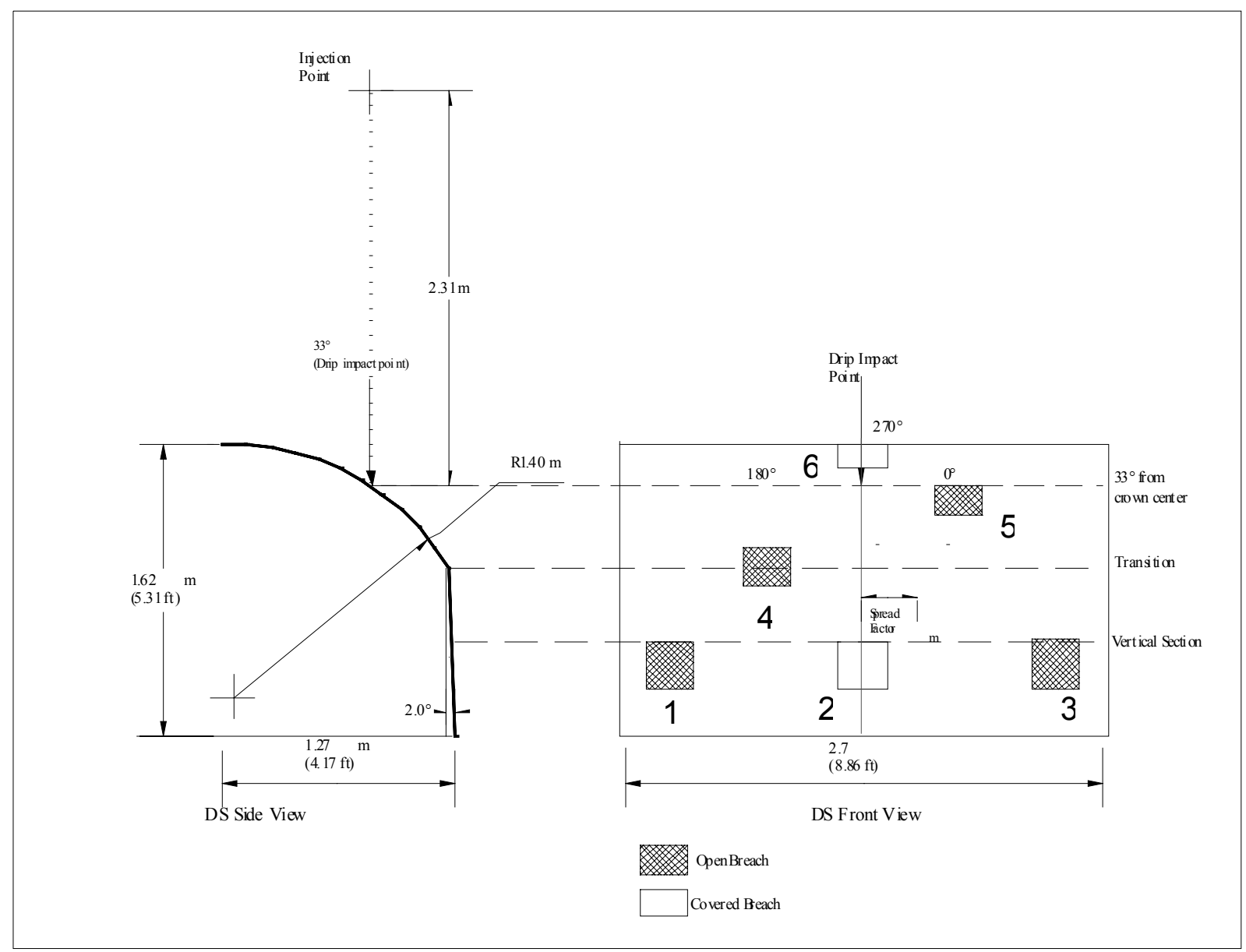

Source: Howard 2002a, p. 62.

Figure 13. Spread Factor Test \#4 Geometry on Smooth DS Surface 


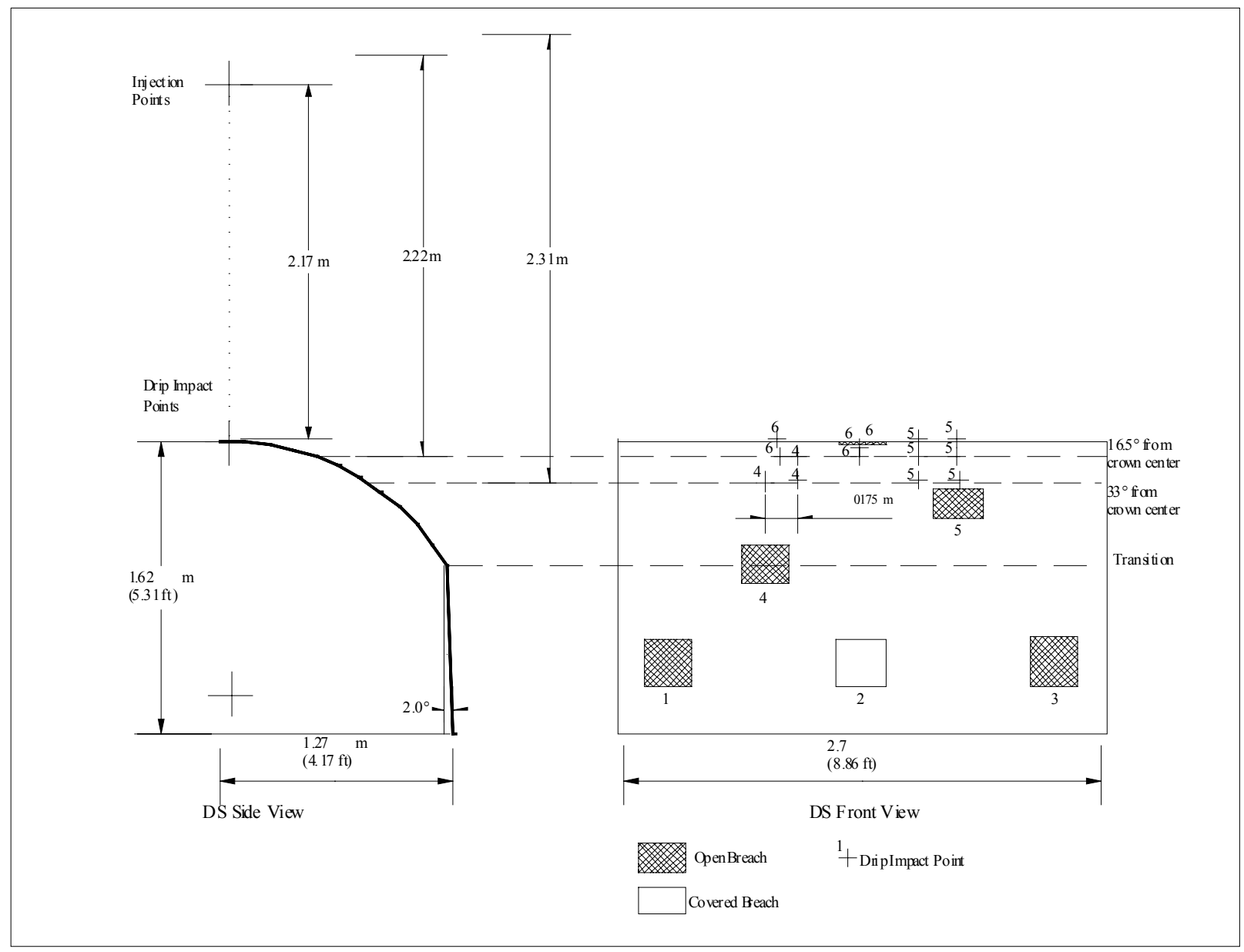

Source: Howard 2002a, p. 68

Figure 14. Single Patch q(splash) Tests on Smooth DS Surface 


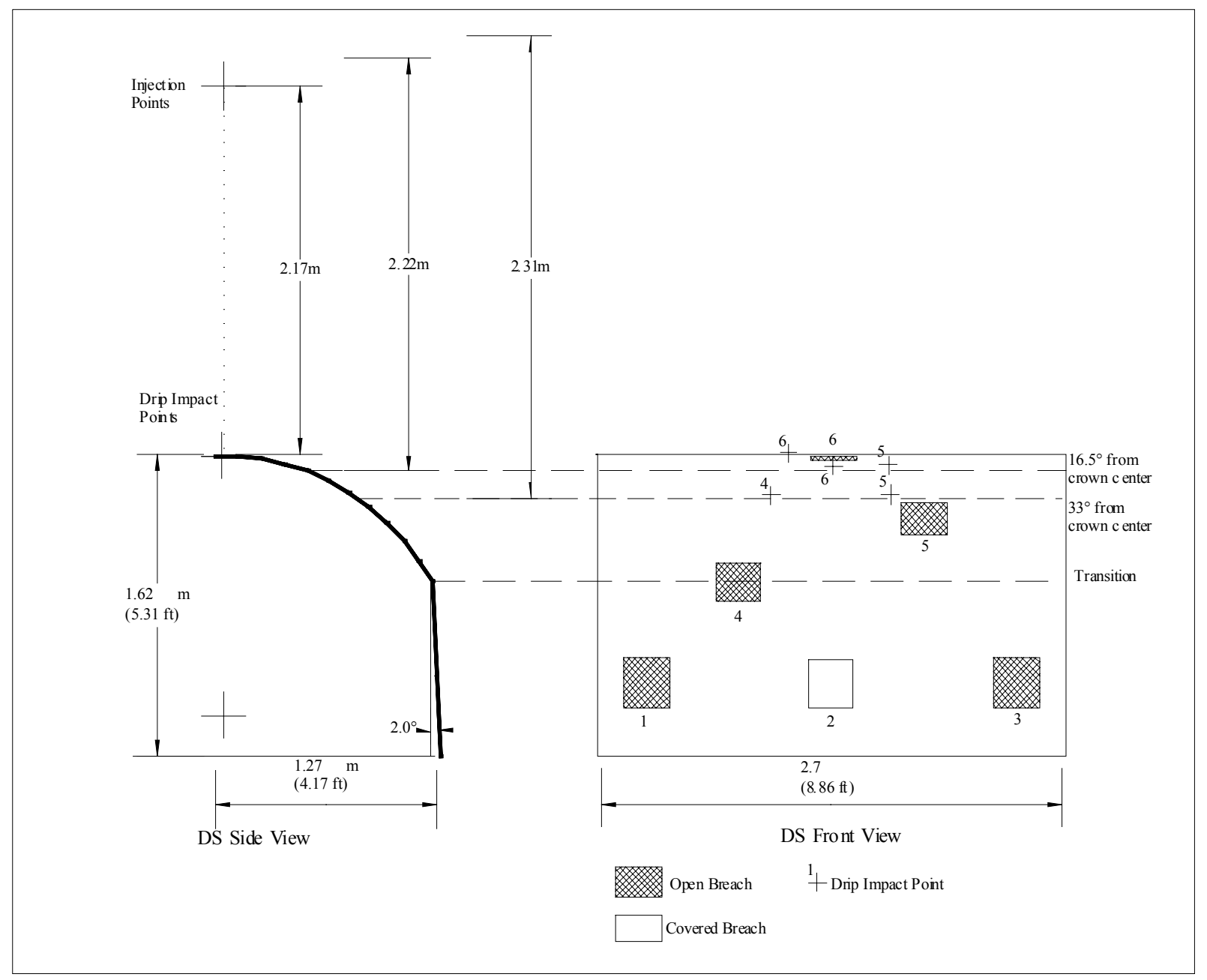

Source: Howard 2002b, p. 92.

Figure 15. Single Patch q(splash) Tests on Rough DS Surface 


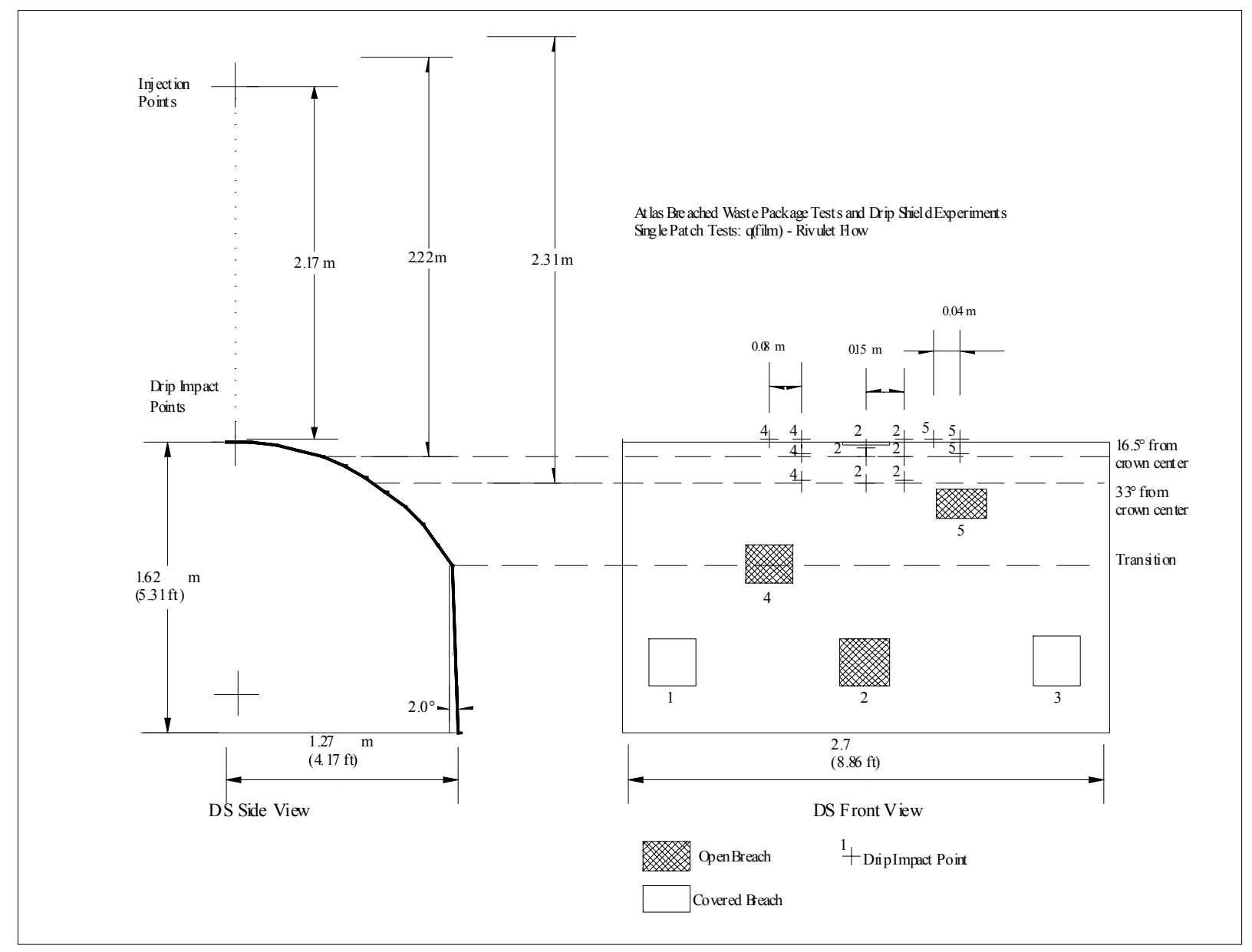

Source: Howard 2002a, p. 110.

Figure 16. Single Patch q (film) Tests on Smooth DS Surface 


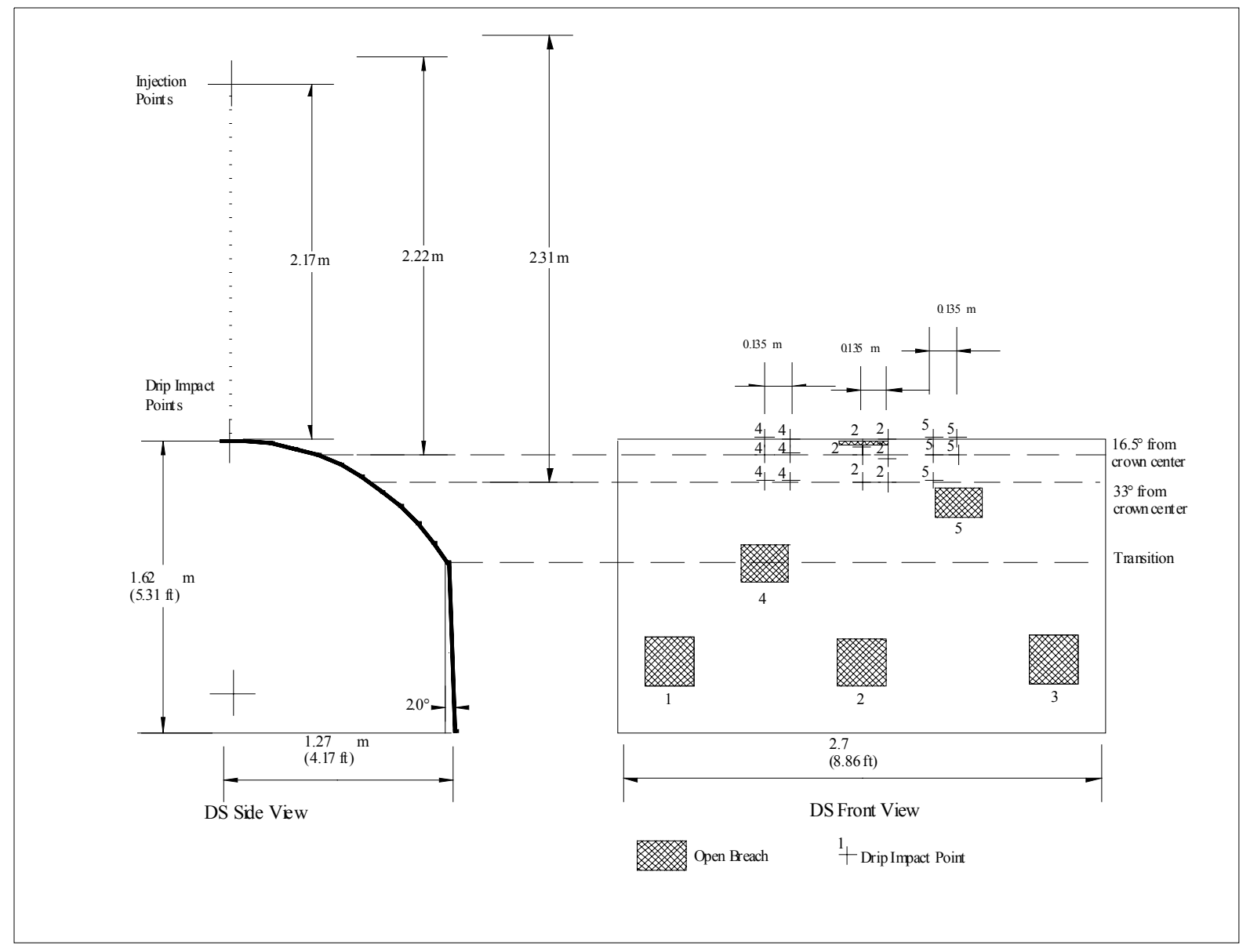

Source: Howard 2002b, p. 107.

Figure 17. Single Patch q(film) Tests on Rough DS Surface 


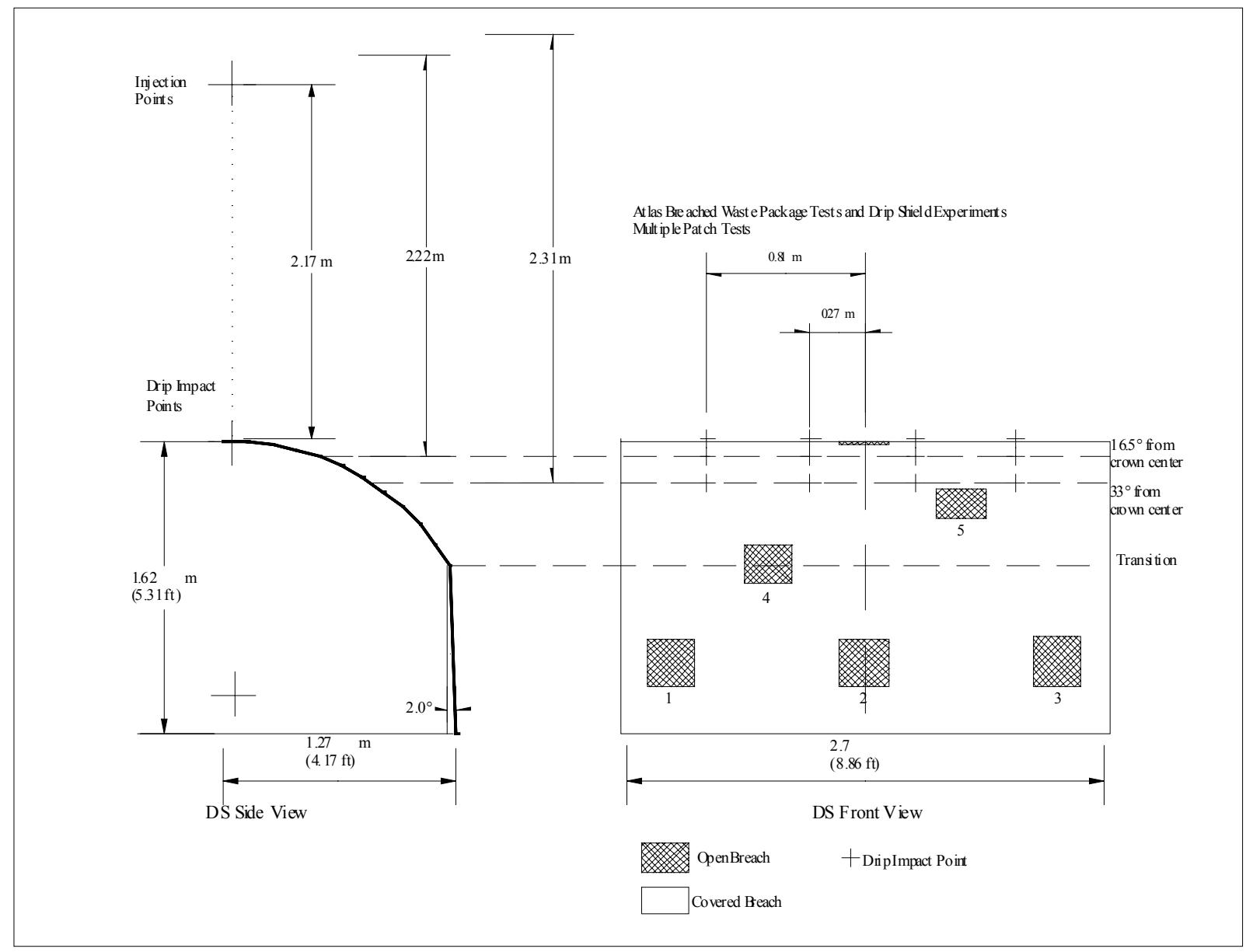

Source: Howard 2002a, p. 140.

Figure 18. Multiple Patch Tests on Smooth and Rough DS Surfaces 


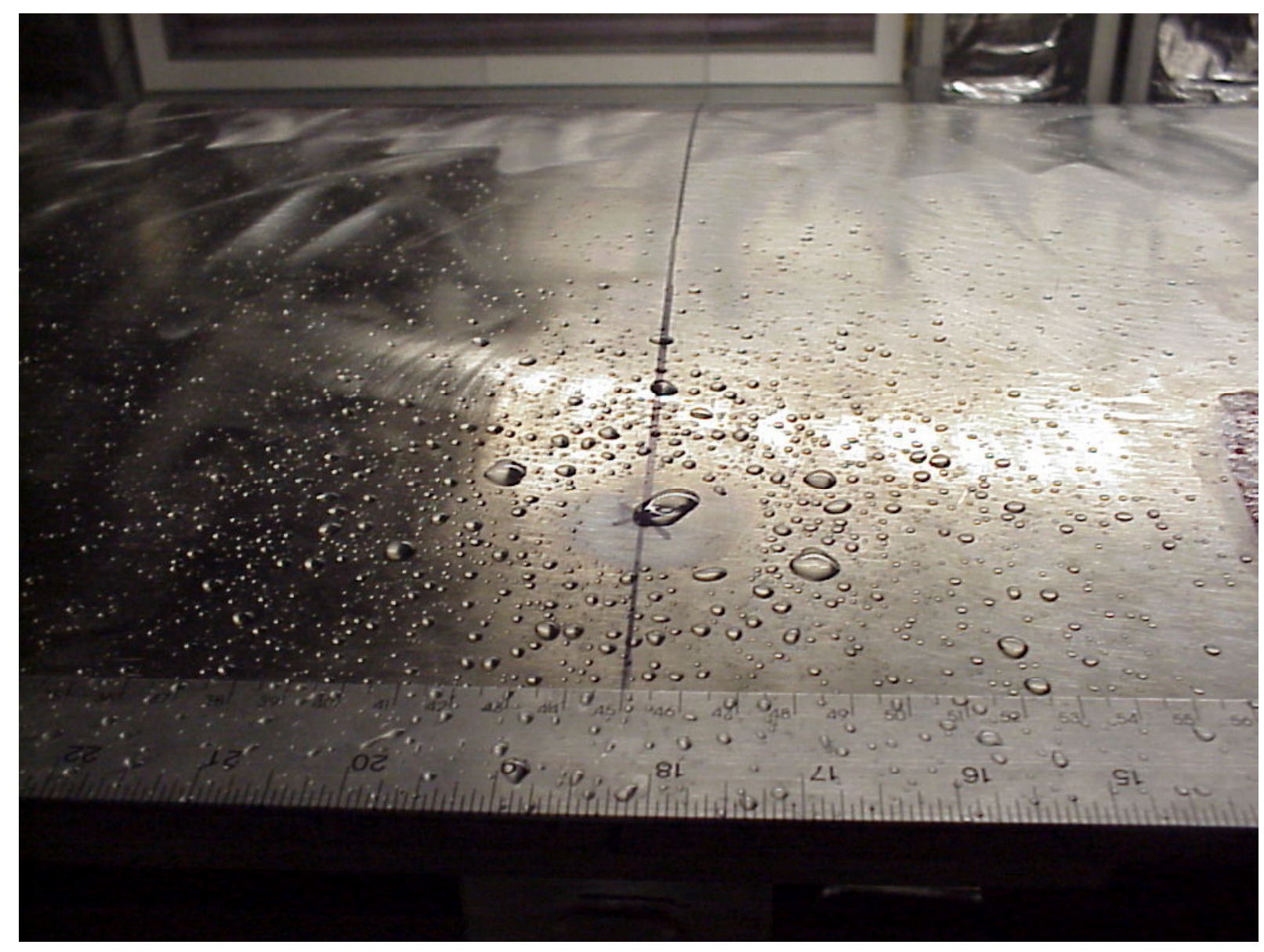

Source: Howard 2002a, p. 44.

Figure 19. Water Droplet Formation on the Smooth DS Surface 


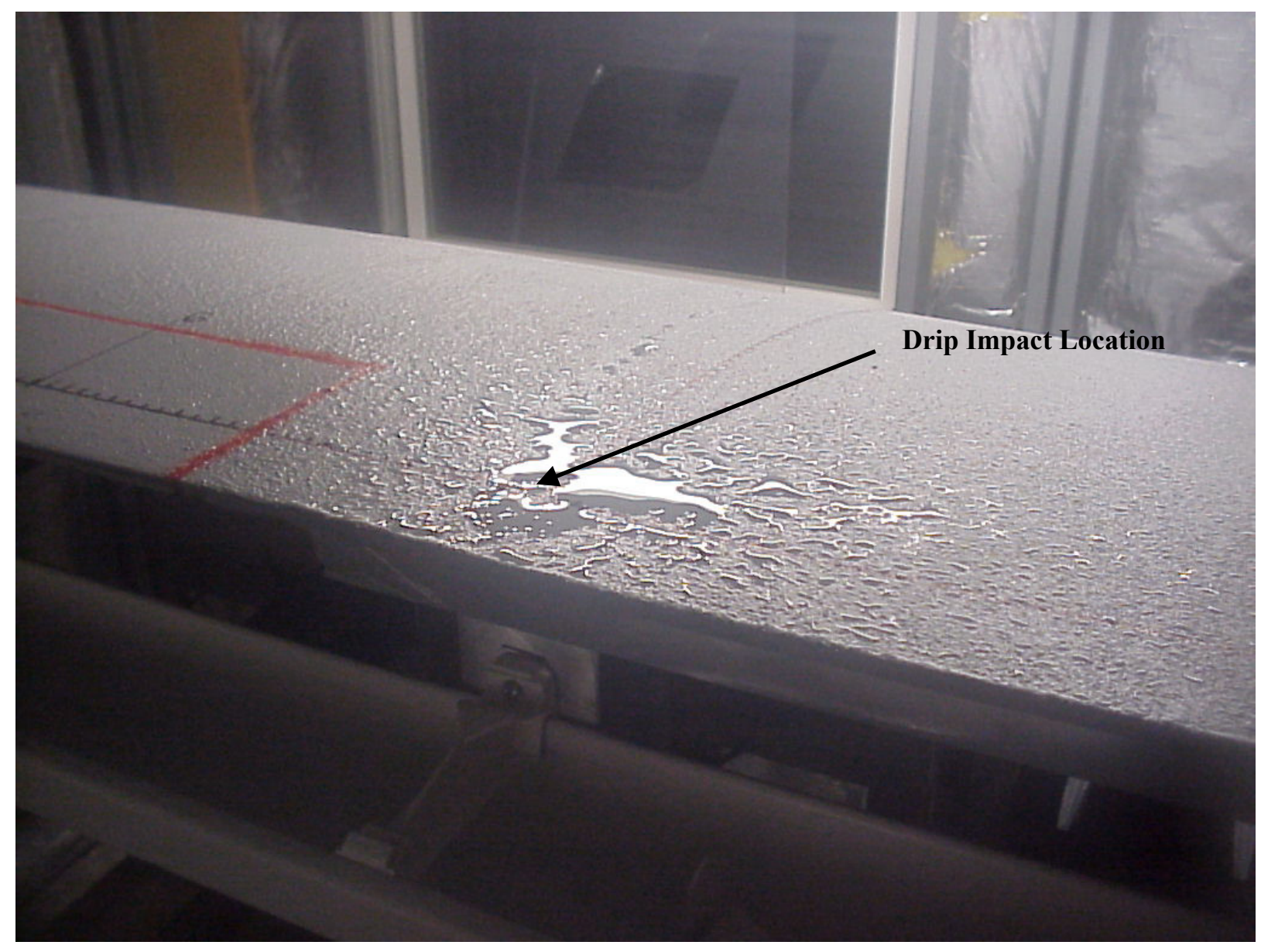

Source: Howard 2002b, p. 76.

Figure 20. Water Pooling on Rough DS Surface 
Table 1 EBS/Repository Design Parameters

\begin{tabular}{|l|l|l|}
\hline \multicolumn{1}{|c|}{ Parameter } & \multicolumn{1}{|c|}{ Parameter Value } & \multicolumn{1}{c|}{ Source } \\
\hline Drip Shield Height & $2521 \mathrm{~mm}$ & CRWMS M\&O 2000a \\
\hline Drip Shield Width & $2512 \mathrm{~mm}$ & CRWMS M\&O 2000a \\
\hline Drip Shield Radius of Curvature & $1.3 \mathrm{~m}$ & CRWMS M\&O 2000a \\
\hline Invert Depth & $0.806 \mathrm{~m}$ & CRWMS M\&O 2000c \\
\hline Drift Diameter & $5.5 \mathrm{~m}$ & CRWMS M\&O 1999 \\
\hline WP Diameter & $1.564 \mathrm{~m}$ & CRWMS M\&O 2000b \\
\hline $\begin{array}{l}\text { Fall Distance From Drift Crown to DS } \\
\text { Crown (see Figure 1) }\end{array}$ & $2173 \mathrm{~mm}$ & $\begin{array}{l}\text { Drift Diameter }- \text { (DS Height }+ \\
\text { Invert Depth) }\end{array}$ \\
\hline
\end{tabular}

Source: BSC (Bechtel SAIC Company) 2002, p. 8.

Table 2. BWPDSE Instrument Accuracy

\begin{tabular}{|l|l|l|}
\hline \multicolumn{1}{|c|}{ Instrument } & \multicolumn{1}{|c|}{ Range } \\
\hline $\begin{array}{l}\text { Temperature/Humidity Probe } \\
\text { (Vaisala HMP 235A) }\end{array}$ & $\begin{array}{l}10-100 \% \mathrm{RH} \\
0-100{ }^{\circ} \mathrm{C}\end{array}$ & $\begin{array}{l}\text { Accuracy } \\
\pm 2 \% \mathrm{RH} @ 10-90 \% \mathrm{RH}, \\
\pm 3.0 \% \mathrm{RH} \text { above } 90 \% \mathrm{RH} ; \\
\text { Temperature } \pm 2.0{ }^{\circ} \mathrm{C}\end{array}$ \\
\hline $\begin{array}{l}\text { Pressure Transducer } \\
\text { (Setra 270) }\end{array}$ & $800-1100 \mathrm{mbar}$ & $\pm 0.05 \%$ Full Scale \\
\hline $\begin{array}{l}\text { Analytical Balance } \\
\text { (Mettler PM4000) }\end{array}$ & $0-4000 \mathrm{~g}$ & $\begin{array}{l}\text { Minimum Accuracy } \\
\pm 0.06 \mathrm{~g}\end{array}$ \\
\hline $\begin{array}{l}\text { Analytical Balance } \\
\text { (Mettler PJ360) }\end{array}$ & $0-300 \mathrm{~g}$ & $\begin{array}{l}\text { Minimum Accuracy } \\
\pm 0.06 \mathrm{~g}\end{array}$ \\
\hline $\begin{array}{l}\text { RTD } \\
\text { (Omega RTD-809) }\end{array}$ & $\begin{array}{l}\text { Calibrated Range: } \\
15,45, \text { and } 100^{\circ} \mathrm{C}\end{array}$ & $\pm 0.3{ }^{\circ} \mathrm{C}$ \\
\hline
\end{tabular}

Source: Howard 2002a, pp. 25-34; Howard 2002c, pp. 223-240. 
Table 3. Water Measurement Descriptions

\begin{tabular}{|l|l|}
\hline \multicolumn{1}{|c|}{ Measurement Type } & \multicolumn{1}{c|}{ Measurement Description } \\
\hline Input Water & $\begin{array}{l}\text { Injection source water in beaker located on Analytical Balance PM4000 (Howard } \\
2002 \mathrm{~b}, \mathrm{p} .3 \text { ) inside the test chamber. RTD probe submerged in water and reading } \\
\text { was taken after } 30 \mathrm{sec} .\end{array}$ \\
\hline DS Beaker Water & $\begin{array}{l}\text { Water in glass beaker placed in contact with the DS surface throughout the test. } \\
\text { RTD probe is placed below water surface in beaker and reading was taken after } \\
30 \text { sec. }\end{array}$ \\
\hline DS Surface Water & $\begin{array}{l}\text { Water film remaining on the DS following a test. RTD probe is placed in contact } \\
\text { with the DS. The probe is not submerged in water, and in most cases only a thin } \\
\text { film is present. Reading is taken after 30 sec. }\end{array}$ \\
\hline Air Temperature & $\begin{array}{l}\text { RTD probe is mounted so that it is not in contact with DS any other surface. } \\
\text { Reading is taken after } 30 \text { sec. }\end{array}$ \\
\hline
\end{tabular}

Source: Howard 2002b, pp. 220-222.

Table 4. BWPDSE Test Matrix

\begin{tabular}{|l|l|}
\hline \multicolumn{1}{|c|}{ Tests Conducted on Smooth DS Surface } & \multicolumn{1}{c|}{ Tests Conducted on Rough DS Surface } \\
\hline Initial/Preliminary Tests: & Initial/Preliminary Tests: \\
Splash Radius (Drip Splash) Test \#1 & Splash Radius (Drip Splash) Test \#1 \\
Splash Radius (Drip Splash) Test \#2 & Splash Radius (Drip Splash) Test \#2 \\
Spread Factor Test \#1 & Splash Radius (Drip Splash) Test \#3 \\
Spread Factor Test \#2 & Splash Radius (Drip Splash) Test \#4 \\
Spread Factor Test \#3 & Splash Radius (Drip Splash) Test \#5 \\
Spread Factor Test \#4 & Spread Factor Test \#1 \\
& Spread Factor Test \#2 \\
\hline Single Patch q(splash) Tests & Spread Factor Test \#3 \\
\hline Single Patch q(film) - Rivulet Flow Tests & Single Patch q(splash) Tests \\
\hline Multiple Patch Tests & Single Patch q(film) - Rivulet Flow Tests \\
\hline
\end{tabular}

Source: Howard 2002a, pp. 39-151; Howard 2002b, pp. 9-219. 
Table 5. Single Patch q(splash) Drip Location on Smooth DS Surface

\begin{tabular}{|c|c|c|}
\hline Patch/Breach & Drip Location & Drop Distance $(\mathrm{m})$ \\
\hline \multirow[t]{4}{*}{ Patch 4} & Patch center, $33^{\circ}$ & 2.31 \\
\hline & $17.5 \mathrm{~cm}$ right of center, $33^{\circ}$ & 2.31 \\
\hline & $17.5 \mathrm{~cm}$ right of center, $16.5^{\circ}$ & 2.22 \\
\hline & Patch center, $16.5^{\circ}$ & 2.22 \\
\hline \multirow[t]{6}{*}{ Patch 5} & Patch center, crown & 2.17 \\
\hline & $17.5 \mathrm{~cm}$ left of center, crown & 2.17 \\
\hline & Patch center, $16.5^{\circ}$ & 2.22 \\
\hline & $17.5 \mathrm{~cm}$ left of center, $16.5^{\circ}$ & 2.22 \\
\hline & Patch center, $33^{\circ}$ & 2.31 \\
\hline & $17.5 \mathrm{~cm}$ left of center, $33^{\circ}$ & 2.31 \\
\hline \multirow[t]{5}{*}{ Patch 6} & $37 \mathrm{~cm}$ left of center, crown & 2.17 \\
\hline & $\begin{array}{l}36.5 \mathrm{~cm} \text { left of center, between patch edge and } \\
16.5^{\circ}\end{array}$ & 2.17 \\
\hline & Patch center, between crown and $16.5^{\circ}$ & 2.17 \\
\hline & $35.5 \mathrm{~cm}$ left of center, $16.5^{\circ}$ & 2.22 \\
\hline & Patch center, $16.5^{\circ}$ & 2.22 \\
\hline
\end{tabular}

Source: Howard 2002a, p. 67.

Table 6. Single Patch Drip Locations on Rough DS Surface

\begin{tabular}{|c|c|c|}
\hline Patch/Breach & Drip Location & Drop Distance (m \\
\hline Patch 4 & $27 \mathrm{~cm}$ right of Patch 4 center, $33^{\circ}$ & 2.31 \\
\hline \multirow[t]{2}{*}{ Patch 5} & $20 \mathrm{~cm}$ left of Patch 5 center, $33^{\circ}$ & 2.31 \\
\hline & $20 \mathrm{~cm}$ left of Patch 5 center, $16.5^{\circ}$ & 2.22 \\
\hline \multirow[t]{3}{*}{ Patch 6} & $20 \mathrm{~cm}$ left of Patch 6 center, Crown & 2.17 \\
\hline & $\begin{array}{l}\text { Center of Patch } 6,15 \mathrm{~cm} \text { forward from } \\
\text { crown along DS center. }\end{array}$ & 2.17 \\
\hline & Center of Patch $6,16.5^{\circ}$ & 2.22 \\
\hline
\end{tabular}

Source: Howard 2002b, p. 90. 
Table 7. Single Patch q(film) Drip Locations on Smooth DS Surface

\begin{tabular}{|c|c|c|}
\hline Patch/Breach & Drip Location & Drop Distance $(\mathrm{m})$ \\
\hline \multirow[t]{6}{*}{ Patch 2} & $15 \mathrm{~cm}$ right of patch center, crown & 2.17 \\
\hline & Patch center, $10.5 \mathrm{~cm}$ forward from crown & 2.17 \\
\hline & Patch center, $33^{\circ}$ & 2.31 \\
\hline & $15 \mathrm{~cm}$ right of patch center, $16.5^{\circ}$ & 2.22 \\
\hline & Patch center, $16.5^{\circ}$ & 2.22 \\
\hline & $15 \mathrm{~cm}$ right of patch center, $33^{\circ}$ & 2.31 \\
\hline \multirow[t]{4}{*}{ Patch 4} & $8 \mathrm{~cm}$ right of patch center, $33^{\circ}$ & 2.31 \\
\hline & $8 \mathrm{~cm}$ right of patch center, crown & 2.17 \\
\hline & Patch center, crown & 2.17 \\
\hline & $8 \mathrm{~cm}$ right of patch center, $16.5^{\circ}$ & 2.22 \\
\hline \multirow[t]{3}{*}{ Patch 5} & Patch center, crown & 2.17 \\
\hline & $4 \mathrm{~cm}$ left of center, crown & 2.17 \\
\hline & $4 \mathrm{~cm}$ left of center, $16.5^{\circ}$ & 2.22 \\
\hline
\end{tabular}

Source: Howard 2002a, p. 109. 
Table 8. Single Patch q(film) Drip Locations on Rough DS Surface

\begin{tabular}{|c|c|c|}
\hline Patch/Breach & Drip Location & Drop Distance (m) \\
\hline \multirow[t]{6}{*}{ Patch 2} & $\begin{array}{l}13.5 \mathrm{~cm} \text { right of patch center, } 10.5 \mathrm{~cm} \\
\text { forward from crown }\end{array}$ & 2.17 \\
\hline & Patch center, $10.5 \mathrm{~cm}$ forward from crown & 2.17 \\
\hline & Patch center, $33^{\circ}$ & 2.31 \\
\hline & $13.5 \mathrm{~cm}$ right of patch center, $16.5^{\circ}$ & 2.22 \\
\hline & Patch center, $16.5^{\circ}$ & 2.22 \\
\hline & $13.5 \mathrm{~cm}$ right of patch center, $33^{\circ}$ & 2.31 \\
\hline \multirow[t]{6}{*}{ Patch 4} & $13.5 \mathrm{~cm}$ right of patch center, $33^{\circ}$ & 2.31 \\
\hline & $13.5 \mathrm{~cm}$ right of patch center, $16.5^{\circ}$ & 2.22 \\
\hline & $13.5 \mathrm{~cm}$ right of patch center, crown & 2.17 \\
\hline & Patch center, $33^{\circ}$ & 2.31 \\
\hline & Patch center, $16.5^{\circ}$ & 2.22 \\
\hline & Patch center, crown & 2.17 \\
\hline \multirow[t]{5}{*}{ Patch 5} & Patch center, crown & 2.17 \\
\hline & Patch center, $16.5^{\circ}$ & 2.22 \\
\hline & $13.5 \mathrm{~cm}$ left of patch center, crown & 2.17 \\
\hline & $13.5 \mathrm{~cm}$ left of patch center, $16.5^{\circ}$ & 2.22 \\
\hline & $13.5 \mathrm{~cm}$ left of patch center, $33^{\circ}$ & 2.31 \\
\hline
\end{tabular}

Source: Howard 2002 b, p. 105.

Table 9. Multiple Patch Drip Locations for Smooth and Rough DS Surface Tests

\begin{tabular}{|c|c|c|c|c|c|}
\hline Crown & $\begin{array}{l}0.81 \mathrm{~m} \text { left of } \\
\text { DS centerline }\end{array}$ & $\begin{array}{l}0.27 \mathrm{~m} \text { left of } \\
\text { DS centerline }\end{array}$ & $\begin{array}{l}0.27 \mathrm{~m} \text { right of } \\
\mathrm{DS} \text { centerline }\end{array}$ & $\begin{array}{l}0.81 \mathrm{~m} \text { right of } \\
\mathrm{DS} \text { centerline }\end{array}$ & $\begin{array}{c}\text { Drip distance: } \\
2.17 \mathrm{~m}\end{array}$ \\
\hline $16.5^{\circ}$ Line & $\begin{array}{l}0.81 \mathrm{~m} \text { left of } \\
\mathrm{DS} \text { centerline }\end{array}$ & $\begin{array}{l}0.27 \mathrm{~m} \text { left of } \\
\mathrm{DS} \text { centerline }\end{array}$ & $\begin{array}{l}0.27 \mathrm{~m} \text { right of } \\
\mathrm{DS} \text { centerline }\end{array}$ & $\begin{array}{l}0.81 \mathrm{~m} \text { right of } \\
\mathrm{DS} \text { centerline }\end{array}$ & $\begin{array}{l}\text { Drip distance: } \\
2.22 \mathrm{~m}\end{array}$ \\
\hline $33^{\circ}$ Line & $\begin{array}{l}0.81 \mathrm{~m} \text { left of } \\
\mathrm{DS} \text { centerline }\end{array}$ & $\begin{array}{l}0.27 \mathrm{~m} \text { left of } \\
\mathrm{DS} \text { centerline }\end{array}$ & $\begin{array}{l}0.27 \mathrm{~m} \text { right of } \\
\mathrm{DS} \text { centerline }\end{array}$ & $\begin{array}{l}0.81 \mathrm{~m} \text { right of } \\
\mathrm{DS} \text { centerline }\end{array}$ & $\begin{array}{l}\text { Drip distance: } \\
2.31 \mathrm{~m}\end{array}$ \\
\hline
\end{tabular}

Source: Howard 2002a, p. 139.

Table 10. Bounding Flow Rate Drip Locations for Smooth and Rough DS Surface Tests

\begin{tabular}{|c|c|c|c|c|}
\hline Crown & $\begin{array}{c}0.54 \text { m left of DS } \\
\text { centerline }\end{array}$ & $\begin{array}{c}0.27 \text { m left of DS } \\
\text { centerline }\end{array}$ & $\begin{array}{c}0.27 \text { m right of DS } \\
\text { centerline }\end{array}$ & $\begin{array}{c}\text { Drip distance: } \\
2.17 \mathrm{~m} \\
\end{array}$ \\
\hline $16.5^{\circ}$ Line & $\begin{array}{l}0.54 \mathrm{~m} \text { left of } \mathrm{DS} \\
\text { centerline }\end{array}$ & $\begin{array}{l}0.27 \mathrm{~m} \text { left of } \mathrm{DS} \\
\text { centerline }\end{array}$ & $\begin{array}{l}0.27 \mathrm{~m} \text { right of } \mathrm{DS} \\
\text { centerline }\end{array}$ & $\begin{array}{l}\text { Drip distance: } \\
2.22 \mathrm{~m}\end{array}$ \\
\hline $33^{\circ}$ Line & $\begin{array}{l}0.54 \mathrm{~m} \text { left of DS } \\
\text { centerline }\end{array}$ & $\begin{array}{l}0.27 \mathrm{~m} \text { left of } \mathrm{DS} \\
\text { centerline }\end{array}$ & $\begin{array}{l}0.27 \mathrm{~m} \text { right of } \mathrm{DS} \\
\text { centerline }\end{array}$ & $\begin{array}{l}\text { Drip distance: } \\
2.31 \mathrm{~m}\end{array}$ \\
\hline
\end{tabular}

Source: Howard 2002b, p. 27. 
INTENTIONALLY LEFT BLANK 
APPENDIX A:

\section{M\&TE Used in the BWPDSE}

\begin{tabular}{|c|c|c|c|c|c|c|}
\hline $\begin{array}{c}\text { Data System } \\
\text { Identifier }\end{array}$ & Description & $\begin{array}{c}\text { Sensor Serial } \\
\text { Number }\end{array}$ & $\begin{array}{l}\text { Sensor } \\
\text { Model }\end{array}$ & Calibration Dates & Conversion & Basis \\
\hline 1-HUM-BDST & $\begin{array}{l}\text { Relative } \\
\text { Humidity }\end{array}$ & T2310004 & $\begin{array}{l}\text { Vaisala } \\
\text { HMP235A }\end{array}$ & $\begin{array}{l}\text { Pre-test Cal Date: } \\
05 / 07 / 02 \\
\text { Due: } 05 / 07 / 03 \\
\text { Post-test Cal Date: } \\
09 / 11 / 2002\end{array}$ & Use display panel & $\begin{array}{l}\text { Bechtel Nevada } \\
\text { Calibration Lab } \\
\text { Cal ID } 998256\end{array}$ \\
\hline 1-TMP-BDST & Air Temperature & T2310004 & $\begin{array}{l}\text { Vaisala } \\
\text { HMP235A }\end{array}$ & $\begin{array}{l}\text { Pre-test Cal Date: } \\
05 / 07 / 02 \\
\text { Due: 05/07/03 } \\
\text { Post-test Cal Date: } \\
\text { 09/11/2002 }\end{array}$ & Use display panel & $\begin{array}{l}\text { Bechtel Nevada } \\
\text { Calibration Lab } \\
\text { Cal ID } 998256\end{array}$ \\
\hline 2-HUM-BDST & $\begin{array}{l}\text { Relative } \\
\text { Humidity }\end{array}$ & T4510008 & $\begin{array}{l}\text { Vaisala } \\
\text { HMP235A }\end{array}$ & $\begin{array}{l}\text { Pre-test Cal Date: } \\
05 / 07 / 02 \\
\text { Due: 05/07/03 } \\
\text { Post-test Cal Date: } \\
\text { 09/13/2002 }\end{array}$ & Use display panel & $\begin{array}{l}\text { Bechtel Nevada } \\
\text { Calibration Lab } \\
\text { Cal ID } 998236\end{array}$ \\
\hline 2-TMP-BDST & Air Temperature & T4510008 & $\begin{array}{l}\text { Vaisala } \\
\text { HMP235A }\end{array}$ & $\begin{array}{l}\text { Pre-test Cal Date: } \\
05 / 07 / 02 \\
\text { Due: } 05 / 07 / 03 \\
\text { Post-test Cal Date: } \\
09 / 13 / 2002\end{array}$ & Use display panel & $\begin{array}{l}\text { Bechtel Nevada } \\
\text { Calibration Lab } \\
\text { Cal ID } 998236\end{array}$ \\
\hline P-IN-BDST & $\begin{array}{l}\text { Test chamber } \\
\text { pressure }\end{array}$ & 416325 & $\begin{array}{l}\text { Setra } \\
270\end{array}$ & $\begin{array}{l}\text { Pre-test Cal Date: } \\
05 / 07 / 02 \\
\text { Due: } 05 / 07 / 03 \\
\text { Post-test Cal Date: } \\
08 / 29 / 2002\end{array}$ & $\begin{array}{l}\mathrm{Y}=0.1 \mathrm{X}+600 \\
\mathrm{X}=\mathrm{mVolt}\end{array}$ & $\begin{array}{l}\text { Bechtel Nevada } \\
\text { Calibration Lab } \\
\text { Cal ID } 007763\end{array}$ \\
\hline P-OUT-BDST & Ambient pressure & 416325 & $\begin{array}{l}\text { Setra } \\
270\end{array}$ & $\begin{array}{l}\text { Pre-test Cal Date: } \\
05 / 07 / 02 \\
\text { Due: } 05 / 07 / 03 \\
\text { Post-test Cal Date: } \\
08 / 29 / 2002\end{array}$ & $\begin{array}{l}Y=0.1 X+600 \\
X=m V o l t\end{array}$ & $\begin{array}{l}\text { Bechtel Nevada } \\
\text { Calibration Lab } \\
\text { Cal ID } 007763\end{array}$ \\
\hline $\begin{array}{l}\text { Ambient RH } \\
\text { (EBS-0429) }\end{array}$ & $\begin{array}{l}\text { Ambient } \\
\text { Relative } \\
\text { Humidity }\end{array}$ & W2320024 & $\begin{array}{l}\text { Vaisala } \\
\text { HMP235A }\end{array}$ & $\begin{array}{l}\text { Pre-test Cal Date: } \\
05 / 07 / 02 \\
\text { Due: } 05 / 07 / 03 \\
\text { Post-test Cal Date: } \\
09 / 11 / 2002\end{array}$ & Use display panel & $\begin{array}{l}\text { Bechtel Nevada } \\
\text { Calibration Lab } \\
\text { Cal ID } 315820\end{array}$ \\
\hline $\begin{array}{l}\text { Ambient Temp. } \\
\text { (EBS-0429) }\end{array}$ & $\begin{array}{l}\text { Ambient } \\
\text { Temperature }\end{array}$ & W2320024 & $\begin{array}{l}\text { Vaisala } \\
\text { HMP235A }\end{array}$ & $\begin{array}{l}\text { Pre-test Cal Date: } \\
05 / 07 / 02 \\
\text { Due: } 05 / 07 / 03 \\
\text { Post-test Cal Date: } \\
09 / 11 / 2002\end{array}$ & Use display panel & $\begin{array}{l}\text { Bechtel Nevada } \\
\text { Calibration Lab } \\
\text { Cal ID } 315820\end{array}$ \\
\hline
\end{tabular}

Source: Howard 2002a, pp. 24-34; Howard 2002c, pp. 83-110. 
M\&TE Used in the BWPDSE, Continued

\begin{tabular}{|c|c|c|c|c|c|c|}
\hline $\begin{array}{c}\text { Data System } \\
\text { Identifier }\end{array}$ & Description & $\begin{array}{c}\text { Sensor Serial } \\
\text { Number }\end{array}$ & $\begin{array}{l}\text { Sensor } \\
\text { Model }\end{array}$ & Calibration Dates & Conversion & Basis \\
\hline Fluke Mulitmeter & $\begin{array}{l}\text { Voltage } \\
\text { (Pressure) } \\
\text { Resistance } \\
\text { (RTD Temp.) }\end{array}$ & 6700202 & Fluke 702 & $\begin{array}{l}\text { Pre-test Cal Date: } \\
06 / 11 / 01 \\
\text { Due: } 06 / 11 / 02 \\
\text { Post-test Cal Date: } \\
06 / 14 / 2002\end{array}$ & Use display panel & $\begin{array}{l}\text { Bechtel Nevada } \\
\text { Calibration Lab } \\
\text { Cal ID } 007462\end{array}$ \\
\hline Fluke Mulitmeter & $\begin{array}{l}\text { Voltage } \\
\text { (Pressure) } \\
\text { Resistance } \\
\text { (RTD Temp.) }\end{array}$ & 6695202 & Fluke 702 & $\begin{array}{l}\text { Pre-test Cal Date: } \\
01 / 16 / 02 \\
\text { Due: } 01 / 16 / 03 \\
\text { Post-test Cal Date: } \\
08 / 28 / 2002\end{array}$ & Use display panel & $\begin{array}{l}\text { Bechtel Nevada } \\
\text { Calibration Lab } \\
\text { Cal ID } 007463\end{array}$ \\
\hline $\begin{array}{l}\text { Analytical } \\
\text { Balance PM4000 }\end{array}$ & $\begin{array}{l}\text { Water mass } \\
\text { Input/ } \\
\text { Collection }\end{array}$ & $\mathrm{J} 48850$ & $\begin{array}{l}\text { Mettler } \\
\text { PM4000 }\end{array}$ & $\begin{array}{l}\text { Pre-test Cal Date: } \\
12 / 27 / 01 \\
\text { Due: } 06 / 27 / 02 \\
\text { Post-test Cal Date: } \\
07 / 29 / 2002\end{array}$ & Use display panel & $\begin{array}{l}\text { Bechtel Nevada } \\
\text { Calibration Lab } \\
\text { Cal ID } 307277\end{array}$ \\
\hline $\begin{array}{l}\text { Analytical } \\
\text { Balance PM4000 }\end{array}$ & $\begin{array}{l}\text { Water mass } \\
\text { Input/Collection }\end{array}$ & J48849 & $\begin{array}{l}\text { Mettler } \\
\text { PM4000 }\end{array}$ & $\begin{array}{l}\text { Pre-testCal Date: } \\
02 / 06 / 02 \\
\text { Due: } 08 / 06 / 02 \\
\text { Post-test Cal Date: } \\
08 / 19 / 2002\end{array}$ & Use display panel & $\begin{array}{l}\text { Bechtel Nevada } \\
\text { Calibration Lab } \\
\text { Cal ID } 307232\end{array}$ \\
\hline $\begin{array}{l}\text { Analytical } \\
\text { Balance PJ360 }\end{array}$ & $\begin{array}{l}\text { Water mass } \\
\text { Collection }\end{array}$ & $\mathrm{H} 78850$ & $\begin{array}{l}\text { Mettler } \\
\text { PJ360 }\end{array}$ & $\begin{array}{l}\text { Cal Date: 02/06/02 } \\
\text { Pre-test Due: } \\
08 / 06 / 03 \\
\text { Post-test Cal Date: } \\
08 / 19 / 2002\end{array}$ & Use display panel & $\begin{array}{l}\text { Bechtel Nevada } \\
\text { Calibration Lab } \\
\text { Cal ID } 301293\end{array}$ \\
\hline DS-RTD-01 & $\begin{array}{l}\text { Temperature } \\
\text { Probe RTD-809 }\end{array}$ & 343 & $\begin{array}{l}\text { Omega } \\
\text { RTD-809 }\end{array}$ & $\begin{array}{l}\text { Pre-test Cal Date: } \\
03 / 27 / 02 \\
\text { Due: } 03 / 27 / 03 \\
\text { Post-test Cal Date: } \\
09 / 05 / 2002\end{array}$ & $\begin{array}{l}\text { Use Fluke } 702 \text { in } \\
\text { RTD mode }\end{array}$ & $\begin{array}{l}\text { Bechtel Nevada } \\
\text { Calibration Lab } \\
\text { Cal ID } 992993\end{array}$ \\
\hline
\end{tabular}

Source: Howard 2002a, pp. 24-34; Howard 2002c, pp. 83-110. 


\section{APPENDIX B: Collection Station Percentage Data}

Description of Collection Station Identifiers

Collection station identifiers are represented in the first column of the data sheets contained in Appendix B, and represents the stations where water was collected and weighed during the BDSWPE. The following provides an explanation of the symbols used to identify collection stations. Gutters are shown and labeled in Figure 9 and breach numbering is shown in Figure 4. Designators for test chamber instrumentation are explained in Figure 7.

$\mathrm{G}-$ Gutters

The gutters were identified with the letter $G$, which is followed by two numbers - the first represents the specific gutter (see Figure 9) and the second the swabbing towel or group of towels used during the collection and weighing process on that particular gutter.

SS - Splash Shields

The splash shields were identified with the letters SS, which is followed by a numbers that represents the specific splash shield (see Figure 9). All towels used to swab the splash shields were grouped into one container for weighing.

B - Breach

The breaches were identified with the letter $\mathrm{B}$, which is followed by a numbers that represents the specific breach (see Figure 4).

DS in - Drip Shield Inside Surface

The drip shield inside surface represents under side of the drip shield where water some times formed rivulets after running around the edges of breaches and by passing the breach collection pans during rare instances.

DS out - Drip Shield Outside Surface

The drip shield outer surface represents the outer surface of the drip shield where water was dripped during the BWPDSE. Some portion of the water dripped during the tests collected and remained on the DS surface and this collection station represents the water collected from the DS surface following the tests.

Other

This collection station represented some instances where a collection deviated from the normal routine or collection stations. In such instances, an explanation is provided on the data sheet attachments in the scientific notebooks. 
Single Patch q(splash) Test Summary and Mass Balance-Smooth DS Surface

Test: Patch 4, Centerline, 16.5

Test Date: $\quad 5 / 21 / 02$

\begin{tabular}{|c|c|c|c|c|c|}
\hline & Initial (g) & Final (g) & Difference (g) & $\%$ of Input & Total Input (g) 139.9 \\
\hline Water Input & 669.7 & 529.8 & 139.9 & $100 \%$ & Total Collected (g) 130 \\
\hline Evap & 158.3 & 158.3 & 0 & & \multirow{2}{*}{ 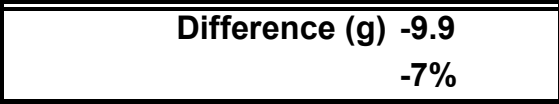 } \\
\hline G1-1 & 7.2 & 11.5 & 4.3 & \multirow[b]{5}{*}{$7.4 \%$} & \\
\hline G1-2 & 7.2 & 12.8 & 5.6 & & \\
\hline G1-3 & & & 0 & & Evaporation \\
\hline G1-4 & & & 0 & & \multirow{2}{*}{$\begin{array}{lr}\text { Evap Pan Tare Wt }(\mathrm{g}) & 67.7 \\
\text { Evaporation }(\mathrm{g}) & 0 \\
\end{array}$} \\
\hline SS-1 & 7.4 & 7.8 & 0.4 & & \\
\hline G2-1 & 7.4 & 7.8 & 0.4 & & \multirow{2}{*}{ \% Evaporation $\quad 0.00 \%$} \\
\hline G2-2 & & & 0 & & \\
\hline G2-3 & & & 0 & & \\
\hline
\end{tabular}

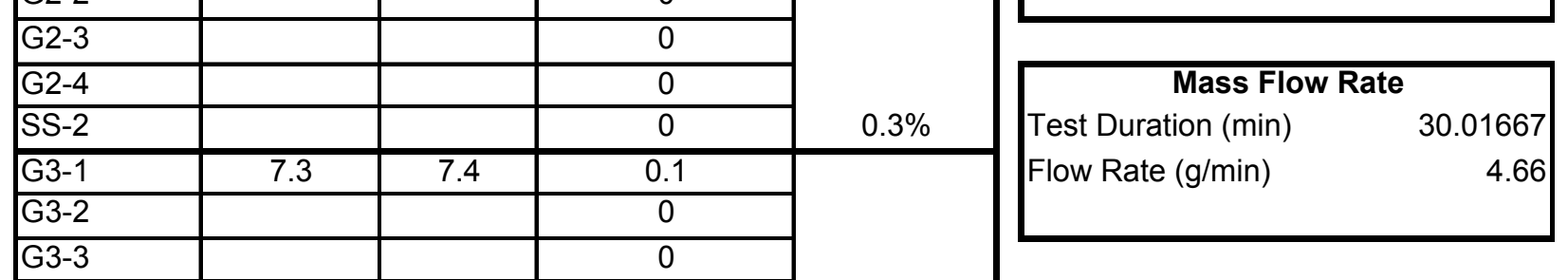

G3-4

SS-3

G4-1

G4-2

G4-3

G4-4

SS-4

\begin{tabular}{|c|c|c|c|c|}
\hline B1 & 106.7 & 106.9 & 0.2 & $0.1 \%$ \\
\hline B2 & & & 0 & $0.0 \%$ \\
\hline B3 & & & 0 & $0.0 \%$ \\
\hline B4 & 106.9 & 191.3 & 84.4 & $60.3 \%$ \\
\hline B5 & 7.2 & 7.4 & 0.2 & $0.1 \%$ \\
\hline B6 & & & 0 & $0.0 \%$ \\
\hline DS in-1 & 7.4 & 7.6 & 0.2 & \multirow[b]{2}{*}{$0.1 \%$} \\
\hline DS in-2 & & & 0 & \\
\hline DS out-1 & 7.2 & 21.8 & 14.6 & \multirow[b]{4}{*}{$24.4 \%$} \\
\hline DS out-2 & 7.3 & 23.2 & 15.9 & \\
\hline DS out-3 & 7.3 & 11.0 & 3.7 & \\
\hline DS out-4 & & & 0 & \\
\hline Other & & & 0 & $0.0 \%$ \\
\hline
\end{tabular}

Test Chamber Environment

\begin{tabular}{|lcccc|}
\hline Initial Conditions & $\mathrm{RH}(\%)$ & Temperature $\left({ }^{\circ} \mathrm{C}\right)$ & Atm. P. (mbar) \\
1-HUM/TMP-BDST & 90.8 & 18.9 & P-IN-BDST & 880 \\
2-HUM/TMP-BDST & 91.3 & 18.7 & & \\
\hline Final Conditions & $\mathrm{RH}(\%)$ & Temperature $\left({ }^{\circ} \mathrm{C}\right)$ & Atm. P. (mbar) \\
1-HUM/TMP-BDST & 96.2 & 19.7 & P-IN-BDST & 887 \\
2-HUM/TMP-BDST & 94.5 & 19.5 & & \\
\hline
\end{tabular}


Single Patch q(splash) Test Summary and Mass Balance-Smooth DS Surface

Test: Patch 4 Centerline, 33

Test Date: $\quad 5 / 20 / 02$

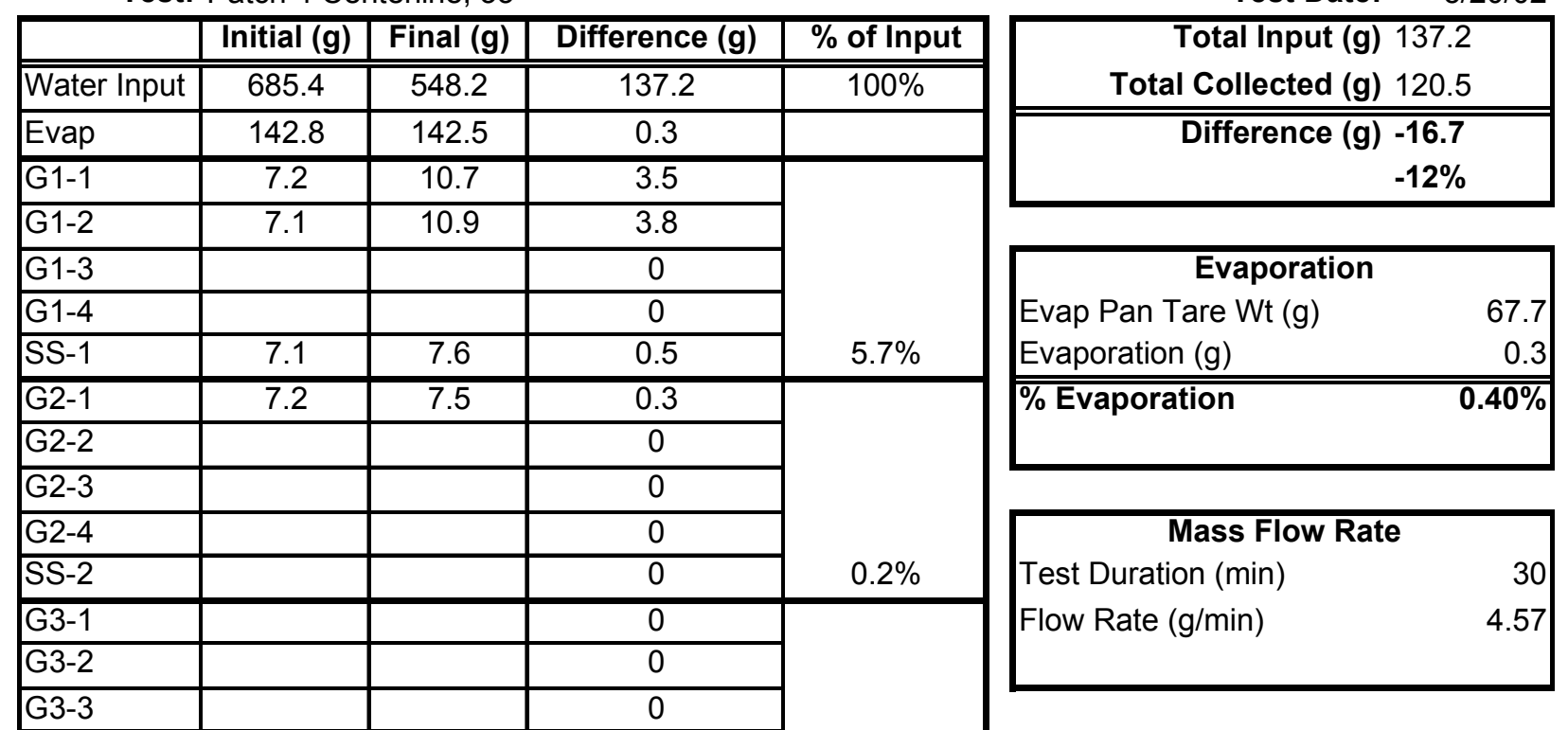

\begin{tabular}{|c|c|c|c|c|}
\hline & & & & \multirow[b]{3}{*}{$0.0 \%$} \\
\hline G3-4 & & & 0 & \\
\hline SS-3 & & & 0 & \\
\hline G4-1 & & & 0 & \multirow[b]{5}{*}{$0.0 \%$} \\
\hline G4-2 & & & $\overline{0}$ & \\
\hline G4-3 & & & 0 & \\
\hline G4-4 & & & 0 & \\
\hline SS-4 & & & 0 & \\
\hline B1 & 106.7 & 106.7 & 0 & $0.0 \%$ \\
\hline B2 & & & 0 & $0.0 \%$ \\
\hline B3 & & & 0 & $0.0 \%$ \\
\hline B4 & 106.8 & 216.7 & 109.9 & $80.1 \%$ \\
\hline B5 & & & 0 & $0.0 \%$ \\
\hline B6 & & & 0 & $0.0 \%$ \\
\hline DS in-1 & 7.2 & 7.3 & 0.1 & \multirow[b]{2}{*}{$0.1 \%$} \\
\hline DS in-2 & & & 0 & \\
\hline DS out-1 & 7.3 & 9.7 & 2.4 & \multirow[b]{4}{*}{$1.7 \%$} \\
\hline DS out-2 & & & 0 & \\
\hline DS out-3 & & & 0 & \\
\hline DS out- 4 & & & 0 & \\
\hline Other & & & 0 & $0.0 \%$ \\
\hline
\end{tabular}

\begin{tabular}{|lcccc|}
\hline \multicolumn{4}{l}{ Test Chamber Environment } & \multicolumn{3}{l|}{} \\
\hline Initial Conditions & $\mathrm{RH}(\%)$ & Temperature $\left({ }^{\circ} \mathrm{C}\right)$ & Atm. P. (mbar) \\
1-HUM/TMP-BDST & 90.5 & 21.2 & P-IN-BDST & 868 \\
2-HUM/TMP-BDST & 89.5 & 20.9 & & \\
\hline Final Conditions & $\mathrm{RH}(\%)$ & Temperature $\left({ }^{\circ} \mathrm{C}\right)$ & & Atm. P. (mbar) \\
1-HUM/TMP-BDST & 88.2 & 21.3 & P-IN-BDST & 869 \\
2-HUM/TMP-BDST & 87.5 & 21 & & \\
\hline
\end{tabular}


Single Patch q(splash) Test Summary and Mass Balance-Smooth DS Surface

Test: Patch 4, $17.5 \mathrm{~cm}$ right of centerline, 16.5

\begin{tabular}{|c|c|c|c|c|c|}
\hline & Initial (g) & Final (g) & Difference (g) & $\%$ of Input & \multirow{2}{*}{$\begin{array}{r}\text { Total Input (g) } 150.8 \\
\text { Total Collected (g) } 129.8\end{array}$} \\
\hline Water Input & 6667.1 & 8516.3 & 10150.8 & \multirow[t]{2}{*}{$100 \%$} & \\
\hline Evap & 158.3 & 158.3 & 0 & & \multirow{2}{*}{ 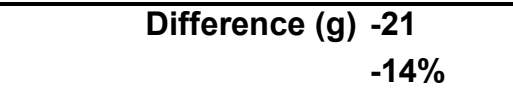 } \\
\hline$\overline{G 1-1}$ & 7.2 & 34.4 & $\overline{27.2}$ & \multirow[b]{5}{*}{$71.6 \%$} & \\
\hline G1-2 & 7.2 & 53.0 & 45.8 & & \\
\hline$\overline{\mathrm{G} 1-3}$ & 7.3 & 40.2 & 32.9 & & Evaporation \\
\hline$\overline{\mathrm{G} 1-4}$ & 7.3 & 9.2 & 1.9 & & \multirow{2}{*}{$\begin{array}{lr}\text { Evap Pan Tare Wt }(\mathrm{g}) & 67.7 \\
\text { Evaporation }(\mathrm{g}) & 0\end{array}$} \\
\hline SS-1 & 7.2 & 7.4 & 0.2 & & \\
\hline$\overline{\mathrm{G} 2-1}$ & & & 0 & \multirow[b]{5}{*}{$0.0 \%$} & \multirow[t]{2}{*}{ \% Evaporation } \\
\hline G2-2 & & & 0 & & \\
\hline G2-3 & & & 0 & & \\
\hline$\overline{G 2-4}$ & & & 0 & & Mass Flow Rate \\
\hline SS-2 & & & 0 & & \multirow{3}{*}{$\begin{array}{l}\text { Test Duration }(\mathrm{min}) \\
\text { Flow Rate }(\mathrm{g} / \mathrm{min})\end{array}$} \\
\hline$\overline{\mathrm{G}} 3-1$ & 7.2 & 7.7 & 0.5 & & \\
\hline G3-2 & & & 0 & & \\
\hline G3-3 & & & 0 & & \\
\hline
\end{tabular}

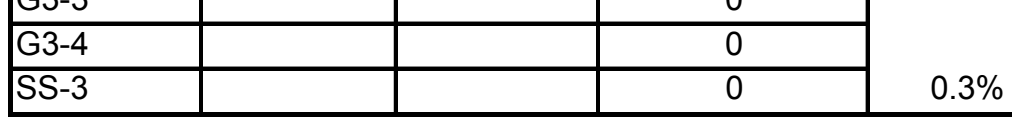

\begin{tabular}{|c|c|c|c|c|}
\hline G4-1 & & & 0 & \multirow[b]{5}{*}{$0.0 \%$} \\
\hline G4-2 & & & 0 & \\
\hline G4-3 & & & 0 & \\
\hline G4-4 & & & 0 & \\
\hline SS-4 & & & 0 & \\
\hline B1 & & & 0 & $0.0 \%$ \\
\hline B2 & & & 0 & $0.0 \%$ \\
\hline B3 & & & 0 & $0.0 \%$ \\
\hline B4 & 106.8 & 108.5 & 1.7 & $1.1 \%$ \\
\hline B5 & 108.7 & 109.3 & 0.6 & $0.4 \%$ \\
\hline B6 & & & 0 & $0.0 \%$ \\
\hline DS in-1 & & & 0 & \multirow[b]{2}{*}{$0.0 \%$} \\
\hline DS in-2 & & & 0 & \\
\hline DS out-1 & 7.6 & 20.4 & 12.8 & \multirow[b]{4}{*}{$12.6 \%$} \\
\hline DS out-2 & 7.3 & 9.9 & 2.6 & \\
\hline DS out-3 & 7.3 & 10.9 & 3.6 & \\
\hline DS out-4 & & & 0 & \\
\hline Other & & & 0 & $0.0 \%$ \\
\hline
\end{tabular}

\begin{tabular}{|lcccc|}
\hline \multicolumn{4}{|l|}{ Test Chamber Environment } & \multicolumn{3}{l|}{} \\
\hline Initial Conditions & $\mathrm{RH}(\%)$ & Temperature $\left({ }^{\circ} \mathrm{C}\right)$ & Atm. P. (mbar) \\
1-HUM/TMP-BDST & 95.9 & 20.3 & P-IN-BDST & 890 \\
2-HUM/TMP-BDST & 94.3 & 19.9 & & \\
\hline Final Conditions & $\mathrm{RH}(\%)$ & Temperature $\left({ }^{\circ} \mathrm{C}\right)$ & Atm. P. (mbar) \\
1-HUM/TMP-BDST & 93 & 20.9 & P-IN-BDST & 889 \\
2-HUM/TMP-BDST & 91.6 & 20.5 & & \\
\hline
\end{tabular}


Single Patch q(splash) Test Summary and Mass Balance-Smooth DS Surface

Test: Patch 4, $17.5 \mathrm{~cm}$ right of centerline, 33

\begin{tabular}{|c|c|c|c|c|}
\hline & Initial (g) & Final (g) & Difference (g) & $\%$ of Input \\
\hline Water Input & 670.3 & 538.8 & 131.5 & $100 \%$ \\
\hline Evap & 143.6 & 143.3 & 0.3 & \\
\hline $\mathrm{G} 1-1$ & 7.5 & 44.2 & 36.7 & \multirow[b]{5}{*}{$79.8 \%$} \\
\hline G1-2 & 7.1 & 57.9 & 50.8 & \\
\hline G1-3 & 7.2 & 20.0 & 12.8 & \\
\hline G1-4 & 7.2 & 8.7 & 1.5 & \\
\hline SS-1 & 7.2 & 10.4 & 3.2 & \\
\hline G2-1 & 7.1 & 7.4 & 0.3 & \multirow[b]{5}{*}{$0.2 \%$} \\
\hline G2-2 & & & 0 & \\
\hline G2-3 & & & 0 & \\
\hline G2-4 & & & 0 & \\
\hline SS-2 & & & 0 & \\
\hline G3-1 & & & 0 & \multirow[b]{5}{*}{$0.0 \%$} \\
\hline G3-2 & & & 0 & \\
\hline G3-3 & & & 0 & \\
\hline G3-4 & & & 0 & \\
\hline SS-3 & & & 0 & \\
\hline G4-1 & & & 0 & \multirow[b]{5}{*}{$0.0 \%$} \\
\hline G4-2 & & & 0 & \\
\hline G4-3 & & & 0 & \\
\hline G4-4 & & & 0 & \\
\hline SS-4 & & & 0 & \\
\hline B1 & 106.2 & 106.7 & 0.5 & $0.4 \%$ \\
\hline B2 & & & 0 & $0.0 \%$ \\
\hline B3 & & & 0 & $0.0 \%$ \\
\hline B4 & 106.7 & 115.7 & 9 & $6.8 \%$ \\
\hline B5 & & & 0 & $0.0 \%$ \\
\hline $\mathrm{B} 6$ & & & 0 & $0.0 \%$ \\
\hline DS in-1 & 7.2 & 7.3 & 0.1 & \multirow[b]{2}{*}{$0.1 \%$} \\
\hline DS in-2 & & & 0 & \\
\hline DS out-1 & 7.3 & 12.7 & 5.4 & \multirow[b]{4}{*}{$4.5 \%$} \\
\hline DS out-2 & 7.1 & 7.6 & 0.5 & \\
\hline DS out- 3 & & & 0 & \\
\hline DS out-4 & & & 0 & \\
\hline Other & & & 0 & $0.0 \%$ \\
\hline
\end{tabular}

Test Date: 5/20/02

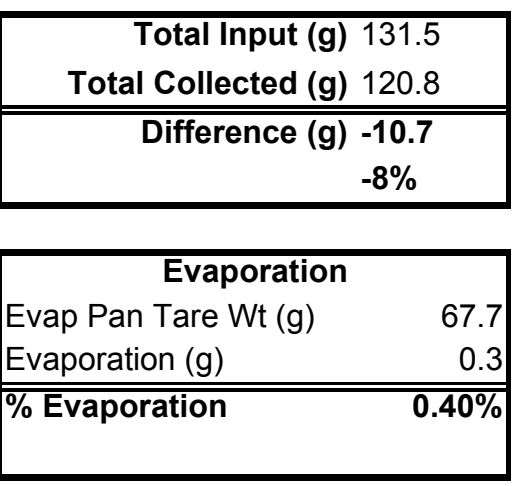

\begin{tabular}{|lr|}
\hline \multicolumn{2}{|c|}{ Mass Flow Rate } \\
Test Duration (min) & 30 \\
Flow Rate (g/min) & 4.38 \\
\hline
\end{tabular}

\begin{tabular}{|lcccc|}
\hline \multicolumn{4}{l}{ Test Chamber Environment } & \multicolumn{5}{l|}{} \\
\hline Initial Conditions & $\mathrm{RH}(\%)$ & Temperature $\left({ }^{\circ} \mathrm{C}\right)$ & Atm. P. (mbar) \\
1-HUM/TMP-BDST & 88.9 & 19.7 & P-IN-BDST & 868 \\
2-HUM/TMP-BDST & 89.3 & 19.5 & & \\
\hline Final Conditions & $\mathrm{RH}(\%)$ & Temperature $\left({ }^{\circ} \mathrm{C}\right)$ & & Atm. P. (mbar) \\
1-HUM/TMP-BDST & 91.1 & 20.7 & P-IN-BDST & 869 \\
2-HUM/TMP-BDST & 87.2 & 20.8 & & \\
\hline
\end{tabular}


Single Patch q(splash) Test Summary and Mass Balance-Smooth DS Surface

Test: Patch 4, centerline, 16.5 *using the 33 degree drip tip.

Test Date: $\quad 5 / 20 / 02$

\begin{tabular}{|l|c|c|c|c|}
\hline & Initial (g) & Final (g) & Difference (g) & \% of Input \\
\hline \hline Water Input & 652.2 & 517.1 & 135.1 & $100 \%$ \\
\hline Evap & 141.1 & 140.8 & 0.3 & \\
\hline
\end{tabular}

\begin{tabular}{|c|}
\hline $\begin{array}{r}\text { Total Input (g) } 135.1 \\
\text { Total Collected }(\mathrm{g}) 101.7\end{array}$ \\
\hline "Difference (g) -33.4 \\
\hline$-25 \%$ \\
\hline
\end{tabular}

\begin{tabular}{|l|c|c|c|}
\hline G1-1 & 7.3 & 9.3 & 2 \\
\hline G1-2 & 7.3 & 7.9 & 0.6 \\
\hline G1-3 & & & 0 \\
\hline G1-4 & & & 0 \\
\hline
\end{tabular}

\begin{tabular}{|l|l|l|l|}
\hline G1-4 & & & 0 \\
\hline SS-1 & & & 0 \\
\hline
\end{tabular}

pan Tare Wt (g) $\quad 67.7$

Evaporation (g)

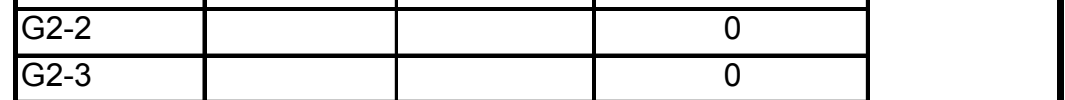

\% Evaporation

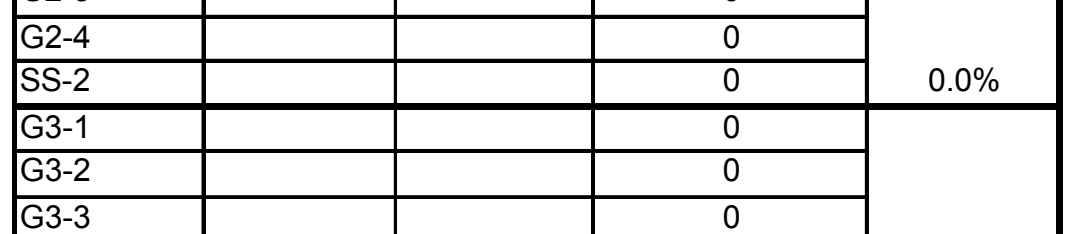

\section{Mass Flow Rate}

Test Duration (min)

Flow Rate (g/min)

\begin{tabular}{|c|c|c|c|c|}
\hline SS-3 & & & 0 & $0.0 \%$ \\
\hline$\overline{\text { G4-1 }}$ & 7.4 & 7.8 & 0.4 & \\
\hline G4-2 & & & 0 & \\
\hline
\end{tabular}

G4-3

G4-4

SS-4

B1

B2

B3

B4

B5

B6

DS in-1

DS in-2

DS out-1

DS out-2

DS out-3

DS out-4

0

Other

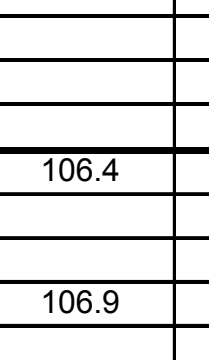

\begin{tabular}{|c|c|c|c|} 
& & 0 & $0.0 \%$ \\
\hline & & 0 & $0.0 \%$ \\
\hline 7.4 & 19.8 & 0 & \multirow{2}{*}{$0.0 \%$} \\
\hline 7.4 & 17.4 & 0 & \multirow{2}{*}{} \\
\cline { 1 - 3 } & & 12.4 & \multirow{2}{*}{$17.2 \%$} \\
\hline & 8.2 & 0.9 & $0.0 \%$ \\
\hline
\end{tabular}

Total Collected

101.7

$75.3 \%$

\begin{tabular}{|lcccc|}
\hline \multicolumn{5}{l}{ Test Chamber Environment } \\
\hline Initial Conditions & $\mathrm{RH}(\%)$ & Temperature $\left({ }^{\circ} \mathrm{C}\right)$ & Atm. P. (mbar) \\
1-HUM/TMP-BDST & 86.7 & 21.5 & P-IN-BDST & 869 \\
2-HUM/TMP-BDST & 83.3 & 21.5 & & \\
\hline Final Conditions & $\mathrm{RH}(\%)$ & Temperature $\left({ }^{\circ} \mathrm{C}\right)$ & Atm. P. (mbar) \\
1-HUM/TMP-BDST & 91 & 21.6 & P-IN-BDST & 869 \\
2-HUM/TMP-BDST & 86.6 & 21.8 & & \\
\hline
\end{tabular}


Single Patch q(splash) Test Summary and Mass Balance-Smooth DS Surface

Test: Patch 5, Centerline, 33, 1 hour

Test Date: $\quad 5 / 16 / 02$

\begin{tabular}{|c|c|c|c|c|c|}
\hline & Initial (g) & Final (g) & Difference $(\mathrm{g})$ & $\%$ of Input & \\
\hline Water Input & 761.0 & 533.8 & 227.2 & $100 \%$ & Total Collected (g) 174.4 \\
\hline Evap & 350.3 & 349.7 & 0.6 & & \multirow{2}{*}{$\begin{array}{r}-52.8 \\
-23 \%\end{array}$} \\
\hline G1-1 & 7.2 & 25.0 & 17.8 & \multirow[b]{5}{*}{$29.1 \%$} & \\
\hline G1-2 & 7.3 & 46.6 & 39.3 & & \\
\hline G1-3 & 7.1 & 10.6 & 3.5 & & Evaporation \\
\hline G1-4 & 7.0 & 12.5 & 5.5 & & Evap Pan Tare Wt (g) \\
\hline SS-1 & & & 0 & & Evaporation (g) \\
\hline G2-1 & & & 0 & & \% Evaporation \\
\hline G2-2 & & & 0 & & \\
\hline G2-3 & & & 0 & & \\
\hline G2-4 & & & 0 & & Mass Flow Rate \\
\hline SS-2 & & & 0 & $0.0 \%$ & Test Duration (min) \\
\hline G3-1 & & & 0 & & Flow Rate (g/min) \\
\hline G3-2 & & & 0 & & \\
\hline G3-3 & & & 0 & & \\
\hline
\end{tabular}

\begin{tabular}{|c|c|c|c|c|}
\hline G3-4 & & & 0 & \\
\hline SS-3 & & & 0 & $0.0 \%$ \\
\hline G4-1 & & & 0 & \multirow[b]{5}{*}{$0.0 \%$} \\
\hline G4-2 & & & 0 & \\
\hline G4-3 & & & 0 & \\
\hline G4-4 & & & 0 & \\
\hline SS-4 & & & 0 & \\
\hline B1 & & & 0 & $0.0 \%$ \\
\hline B2 & & & 0 & $0.0 \%$ \\
\hline B3 & 105.5 & 106.1 & 0.6 & $0.3 \%$ \\
\hline B4 & 107.0 & 107.0 & 0 & $0.0 \%$ \\
\hline B5 & 108.8 & 209.6 & 100.8 & $44.4 \%$ \\
\hline B6 & & & 0 & $0.0 \%$ \\
\hline DS in-1 & 7.3 & 8.6 & 1.3 & \multirow[b]{2}{*}{$0.6 \%$} \\
\hline DS in-2 & & & 0 & \\
\hline DS out-1 & 7.0 & 12.1 & 5.1 & \multirow[b]{4}{*}{$2.5 \%$} \\
\hline DS out-2 & 6.8 & 7.3 & 0.5 & \\
\hline DS out-3 & & & 0 & \\
\hline DS out-4 & & & 0 & \\
\hline Other & & & 0 & $0.0 \%$ \\
\hline
\end{tabular}

\begin{tabular}{|lcccc|}
\hline \multicolumn{5}{ll}{ Test Chamber Environment } \\
\hline Initial Conditions & $\mathrm{RH}(\%)$ & Temperature $\left({ }^{\circ} \mathrm{C}\right)$ & Atm. P. (mbar) \\
1-HUM/TMP-BDST & 82 & 22.2 & P-IN-BDST & 865 \\
2-HUM/TMP-BDST & 84.5 & 21.7 & & \\
\hline Final Conditions & $\mathrm{RH}(\%)$ & Temperature $\left({ }^{\circ} \mathrm{C}\right)$ & Atm. P. (mbar) \\
1-HUM/TMP-BDST & 73.9 & 23.9 & P-IN-BDST & 861 \\
2-HUM/TMP-BDST & 68.5 & 24 & & \\
\hline
\end{tabular}


Single Patch q(splash) Test Summary and Mass Balance-Smooth DS Surface

Test: Patch 5, Centerline, 33, 30 min

Test Date: $\quad 5 / 16 / 02$

\begin{tabular}{|c|c|c|c|c|c|}
\hline & Initial (g) & Final (g) & Difference $(\mathrm{g})$ & $\%$ of Input & \multirow{2}{*}{$\begin{aligned} \text { Total Input (g) } & 144.0 \\
\text { Total Collected (g) } & 107\end{aligned}$} \\
\hline Water Input & 621.7 & 40 & 10144 & \multirow[t]{2}{*}{$63 \%$} & \\
\hline Evap & 143.0 & 142.6 & 0.4 & & \multirow{2}{*}{ 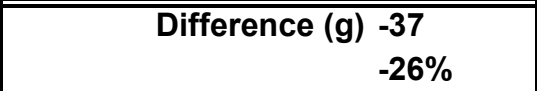 } \\
\hline G1-1 & 7.1 & 16.1 & 9 & \multirow[b]{5}{*}{$7.1 \%$} & \\
\hline$\overline{\mathrm{G} 1-2}$ & 7.2 & 11.4 & 4.2 & & \\
\hline G1-3 & $\overline{7.2}$ & 10.2 & 3 & & Evaporation \\
\hline$\overline{\mathrm{G} 1-4}$ & & & 0 & & Evap Pan Tare Wt (g) \\
\hline SS-1 & & & 0 & & Evaporation (g) \\
\hline$\overline{\mathrm{G} 2-1}$ & & & 0 & \multirow[b]{5}{*}{$0.0 \%$} & \multirow{2}{*}{ \% Evaporation $\quad 0.53 \%$} \\
\hline $\bar{G} 2-2$ & & & 0 & & \\
\hline $\bar{G} 2-3$ & & & 0 & & \\
\hline $\bar{G} 2-4$ & & & 0 & & Mass Flow Rate \\
\hline SS-2 & & & 0 & & \multirow{3}{*}{$\begin{array}{l}\text { Test Duration }(\mathrm{min}) \\
\text { Flow Rate }(\mathrm{g} / \mathrm{min})\end{array}$} \\
\hline G3-1 & & & 0 & & \\
\hline G3-2 & & & 0 & & \\
\hline G3-3 & & & 0 & & \\
\hline
\end{tabular}

\begin{tabular}{|c|c|c|c|c|}
\hline & & & & \multirow[b]{3}{*}{$0.0 \%$} \\
\hline G3-4 & & & 0 & \\
\hline SS-3 & & & 0 & \\
\hline G4-1 & & & 0 & \multirow[b]{5}{*}{$0.0 \%$} \\
\hline G4-2 & & & 0 & \\
\hline G4-3 & & & 0 & \\
\hline G4-4 & & & 0 & \\
\hline SS-4 & & & 0 & \\
\hline B1 & & & 0 & $0.0 \%$ \\
\hline $\mathrm{B} 2$ & & & 0 & $0.0 \%$ \\
\hline B3 & & & 0 & $0.0 \%$ \\
\hline B4 & & & 0 & $0.0 \%$ \\
\hline B5 & 108.8 & 195.3 & 86.5 & $38.1 \%$ \\
\hline $\mathrm{B} 6$ & & & 0 & $0.0 \%$ \\
\hline DS in-1 & 7.3 & 8.0 & 0.7 & \multirow[b]{2}{*}{$0.3 \%$} \\
\hline DS in-2 & & & 0 & \\
\hline DS out-1 & 7.2 & 10.6 & 3.4 & \multirow[b]{4}{*}{$1.6 \%$} \\
\hline DS out-2 & 7.4 & 7.6 & 0.2 & \\
\hline DS out-3 & & & 0 & \\
\hline DS out-4 & & & 0 & \\
\hline Other & & & $\overline{0}$ & $0.0 \%$ \\
\hline
\end{tabular}

\begin{tabular}{|lcccc|}
\hline \multicolumn{7}{l}{ Test Chamber Environment } & & & \\
\hline Initial Conditions & $\mathrm{RH}(\%)$ & Temperature $\left({ }^{\circ} \mathrm{C}\right)$ & Atm. P. (mbar) \\
1-HUM/TMP-BDST & 85.1 & 24.1 & P-IN-BDST & 854 \\
2-HUM/TMP-BDST & 83.5 & 24 & & \\
\hline Final Conditions & $\mathrm{RH}(\%)$ & Temperature $\left({ }^{\circ} \mathrm{C}\right)$ & & Atm. P. (mbar) \\
1-HUM/TMP-BDST & 88.5 & 24.1 & P-IN-BDST & 871 \\
2-HUM/TMP-BDST & 86.6 & 23.8 & & \\
\hline
\end{tabular}


Single Patch q(splash) Test Summary and Mass Balance-Smooth DS Surface

Test: Patch 5, Centerline, 16.5

\begin{tabular}{|c|c|c|c|c|}
\hline & Initial & Final & Difference & $\%$ of Input \\
\hline Water Input & 676.1 & 551.3 & 124.8 & $100 \%$ \\
\hline Evap & 149.6 & 149.5 & 0.1 & \\
\hline$\overline{G 1-1}$ & 7.2 & 9.9 & 2.7 & \multirow[b]{5}{*}{$2.7 \%$} \\
\hline G1-2 & 7.1 & 7.8 & 0.7 & \\
\hline G1-3 & & & 0 & \\
\hline G1-4 & & & 0 & \\
\hline SS-1 & & & 0 & \\
\hline G2-1 & & & 0 & \multirow[b]{5}{*}{$0.0 \%$} \\
\hline G2-2 & & & 0 & \\
\hline G2-3 & & & 0 & \\
\hline G2-4 & & & 0 & \\
\hline SS-2 & & & 0 & \\
\hline G3-1 & $\overline{7.3}$ & 7.6 & 0.3 & \multirow[b]{5}{*}{$0.2 \%$} \\
\hline G3-2 & & & 0 & \\
\hline G3-3 & & & 0 & \\
\hline G3-4 & & & 0 & \\
\hline SS-3 & & 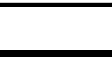 & 0 & \\
\hline$\overline{\mathrm{G}} 4-1$ & 7.2 & 7.7 & 0.5 & \multirow[b]{5}{*}{$0.4 \%$} \\
\hline G4-2 & & & 0 & \\
\hline G4-3 & & & 0 & \\
\hline G4-4 & & & 0 & \\
\hline SS-4 & & & 0 & \\
\hline B1 & & & 0 & $0.0 \%$ \\
\hline B2 & & & 0 & $0.0 \%$ \\
\hline B3 & 105.8 & 106.1 & 0.3 & $0.2 \%$ \\
\hline B4 & & & 0 & $0.0 \%$ \\
\hline B5 & 108.6 & 199.1 & 90.5 & $72.5 \%$ \\
\hline B6 & & & 0 & $0.0 \%$ \\
\hline DS in-1 & & & 0 & \multirow[b]{2}{*}{$0.0 \%$} \\
\hline DS in-2 & & & 0 & \\
\hline DS out-1 & 7.2 & 14.4 & 7.2 & \multirow[b]{4}{*}{$8.2 \%$} \\
\hline DS out-2 & 7.0 & 10.0 & 3 & \\
\hline DS out-3 & & & 0 & \\
\hline DS out-4 & & & 0 & \\
\hline Other & 7.3 & 7.7 & 0.4 & $0.3 \%$ \\
\hline
\end{tabular}

Test Date: $\quad 5 / 22 / 02$

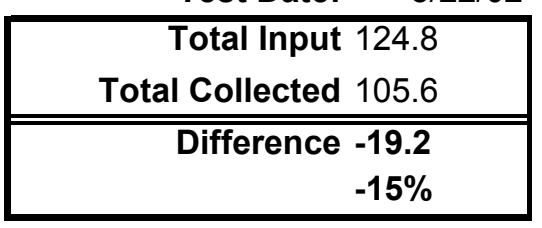

\begin{tabular}{|lr|}
\hline \multicolumn{2}{|c|}{ Evaporation } \\
Evap Pan Tare Wt, g & 66.7 \\
Evaporation, g & 0.1 \\
\hline \% Evaporation & $\mathbf{0 . 1 2} \%$ \\
\hline
\end{tabular}

\begin{tabular}{|lr|}
\hline \multicolumn{2}{|c|}{ Mass Flow Rate } \\
Test Duration, min & 30 \\
Flow Rate, g/min & 4.16 \\
\hline
\end{tabular}

Test Chamber Environment

\begin{tabular}{|lcccc|}
\hline Initial Conditions & $\mathrm{RH}(\%)$ & Temperature $\left({ }^{\circ} \mathrm{C}\right)$ & & Atm. P. (mbar) \\
1-HUM/TMP-BDST & 89.3 & 18.8 & P-IN-BDST & 881 \\
2-HUM/TMP-BDST & 89.4 & 18.8 & & \\
\hline Final Conditions & $\mathrm{RH}(\%)$ & Temperature $\left({ }^{\circ} \mathrm{C}\right)$ & & Atm. P. (mbar) \\
1-HUM/TMP-BDST & 90.4 & 18.8 & P-IN-BDST & 881 \\
2-HUM/TMP-BDST & 89.8 & 18.7 & & \\
\hline
\end{tabular}


Single Patch q(splash) Test Summary and Mass Balance-Smooth DS Surface

Test: Patch 5, Centerline, Crown

\begin{tabular}{|c|c|c|c|c|}
\hline & Initial & Final & Difference & $\%$ of Input \\
\hline Water Input & 672.9 & 535.5 & 137.4 & $100 \%$ \\
\hline Evap & 149.3 & 149.0 & 0.3 & \\
\hline$\overline{G 1-1}$ & 7.3 & 8.4 & 1.1 & \multirow[b]{5}{*}{$3.5 \%$} \\
\hline G1-2 & 7.3 & 11.0 & 3.7 & \\
\hline$\overline{G 1-3}$ & & & 0 & \\
\hline$\overline{G 1-4}$ & & & 0 & \\
\hline SS-1 & & & 0 & \\
\hline$\overline{\mathrm{G} 2-1}$ & & & 0 & \multirow[b]{5}{*}{$0.0 \%$} \\
\hline G2-2 & & & 0 & \\
\hline$\overline{\mathrm{G} 2-3}$ & & & 0 & \\
\hline $\bar{G} 2-4$ & & & 0 & \\
\hline SS-2 & & & 0 & \\
\hline$\overline{\text { G3-1 }}$ & $\overline{7.4}$ & 34.6 & 27.2 & \multirow[b]{5}{*}{$35.9 \%$} \\
\hline G3-2 & 7.2 & 27.0 & 19.8 & \\
\hline G3-3 & 7.5 & 8.0 & 0.5 & \\
\hline G3-4 & & & 0 & \\
\hline SS-3 & 7.4 & 9.2 & 1.8 & \\
\hline$\overline{G 4-1}$ & 7.3 & 7.7 & 0.4 & \multirow[b]{5}{*}{$0.3 \%$} \\
\hline G4-2 & & & 0 & \\
\hline G4-3 & & & 0 & \\
\hline$\overline{G 4-4}$ & & & 0 & \\
\hline SS-4 & & & 0 & \\
\hline$B 1$ & & & 0 & $0.0 \%$ \\
\hline B2 & & & 0 & $0.0 \%$ \\
\hline B3 & 105.5 & 106.0 & 0.5 & $0.4 \%$ \\
\hline B4 & & & 0 & $0.0 \%$ \\
\hline B5 & 108.7 & 124.2 & 15.5 & $11.3 \%$ \\
\hline $\mathrm{B} 6$ & 108.0 & 108.6 & 0.6 & $0.4 \%$ \\
\hline$\overline{D S}$ in-1 & & & 0 & \multirow[b]{2}{*}{$0.0 \%$} \\
\hline DS in-2 & & & 0 & \\
\hline DS out-1 & 7.1 & 43.0 & 35.9 & \multirow[b]{4}{*}{$34.4 \%$} \\
\hline DS out-2 & 7.4 & 16.1 & 8.7 & \\
\hline DS out-3 & 7.4 & 10.0 & 2.6 & \\
\hline DS out-4 & & & 0 & \\
\hline Other & & & 0 & $0.0 \%$ \\
\hline
\end{tabular}

Test Date: $\quad 5 / 22 / 02$

Total Input 137.4

Total Collected 118.3

Difference -19.1

$-14 \%$

\begin{tabular}{|lr|}
\hline \multicolumn{2}{|c|}{ Evaporation } \\
Evap Pan Tare Wt, g & 66.7 \\
Evaporation, g & 0.3 \\
\hline \% Evaporation & $\mathbf{0 . 3 6 \%}$ \\
\hline
\end{tabular}

\begin{tabular}{|lr|}
\hline \multicolumn{2}{|c|}{ Mass Flow Rate } \\
Test Duration, min & 30 \\
Flow Rate, g/min & 4.58 \\
\hline
\end{tabular}

\begin{tabular}{|lcccc|}
\hline \multicolumn{7}{l}{ Test Chamber Environment } & & & \\
\hline Initial Conditions & $\mathrm{RH}(\%)$ & Temperature $\left({ }^{\circ} \mathrm{C}\right)$ & Atm. P. (mbar) \\
1-HUM/TMP-BDST & 92.4 & 20.3 & P-IN-BDST & 877 \\
2-HUM/TMP-BDST & 89.4 & 20.2 & & \\
\hline Final Conditions & $\mathrm{RH}(\%)$ & Temperature $\left({ }^{\circ} \mathrm{C}\right)$ & & Atm. P. (mbar) \\
1-HUM/TMP-BDST & 89.8 & 20.1 & P-IN-BDST & 882 \\
2-HUM/TMP-BDST & 84.4 & 20.2 & & \\
\hline
\end{tabular}

SN-M\&O-SCI-043-V1, pages 96-97 
Single Patch q(splash) Test Summary and Mass Balance-Smooth DS Surface

Test: Patch 5, $17.5 \mathrm{~cm}$ leftt of patch centerline, 16.5

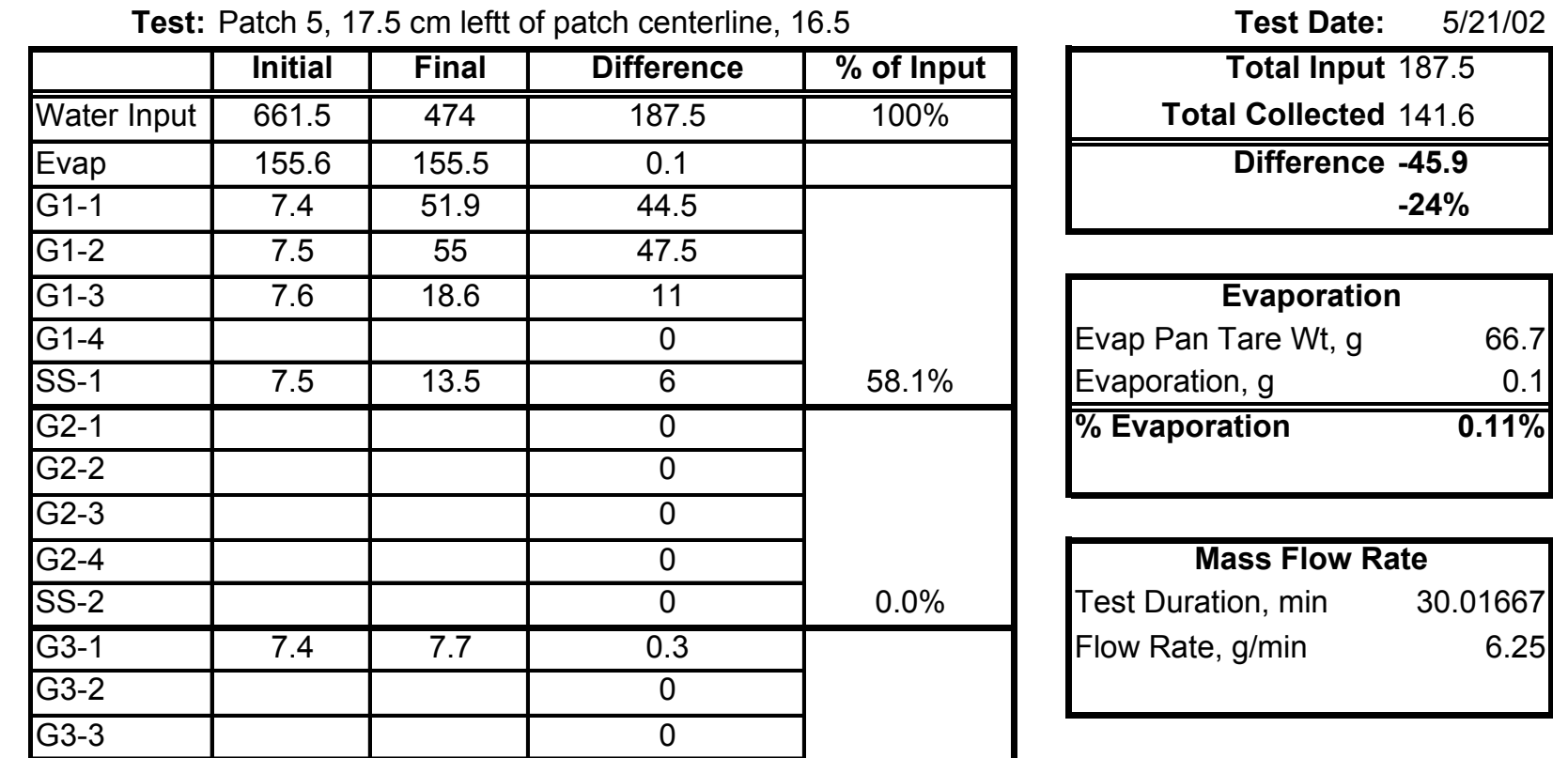

\begin{tabular}{|c|c|c|c|c|}
\hline G3-4 & & & 0 & \\
\hline SS-3 & & & 0 & $0.2 \%$ \\
\hline G4-1 & 7.4 & 7.7 & 0.3 & \multirow[b]{5}{*}{$0.2 \%$} \\
\hline G4-2 & & & 0 & \\
\hline G4-3 & & & 0 & \\
\hline G4-4 & & & 0 & \\
\hline SS-4 & & & 0 & \\
\hline B1 & & & 0 & $0.0 \%$ \\
\hline B2 & & & 0 & $0.0 \%$ \\
\hline B3 & & & 0 & $0.0 \%$ \\
\hline $\bar{B} 4$ & & & 0 & $0.0 \%$ \\
\hline B5 & 109.1 & 111.7 & 2.6 & $1.4 \%$ \\
\hline $\bar{B} 6$ & 108.4 & 108.7 & 0.3 & $0.2 \%$ \\
\hline DS in-1 & 7.5 & 30.7 & 23.2 & \multirow[b]{2}{*}{$15.5 \%$} \\
\hline$\overline{D S}$ in-2 & 7.4 & 13.3 & 5.9 & \\
\hline$\overline{D S}$ out-1 & & & 0 & \multirow[b]{4}{*}{$0.0 \%$} \\
\hline DS out-2 & & & 0 & \\
\hline DS out-3 & & & 0 & \\
\hline DS out-4 & & & 0 & \\
\hline Other & & & 0 & $0.0 \%$ \\
\hline
\end{tabular}

\begin{tabular}{|lcccc|}
\hline \multicolumn{7}{l}{ Test Chamber Environment } & & & \\
\hline Initial Conditions & $\mathrm{RH}(\%)$ & Temperature $\left({ }^{\circ} \mathrm{C}\right)$ & & Atm. P. (mbar) \\
1-HUM/TMP-BDST & 90 & 21.3 & P-IN-BDST & 882 \\
2-HUM/TMP-BDST & 87.3 & 21.3 & & \\
\hline Final Conditions & $\mathrm{RH}(\%)$ & Temperature $\left({ }^{\circ} \mathrm{C}\right)$ & & Atm. P. (mbar) \\
1-HUM/TMP-BDST & 91.3 & 20.7 & P-IN-BDST & 895 \\
2-HUM/TMP-BDST & 88.2 & 20.8 & & \\
\hline
\end{tabular}

SN-M\&O-SCl-043-V1, pages 90-91 
Single Patch q(splash) Test Summary and Mass Balance-Smooth DS Surface

Test: Patch 5, $17.5 \mathrm{~cm}$ left of patch centerline, Crown

\begin{tabular}{|c|c|c|c|c|}
\hline & Initial & Final & Difference & $\%$ of Input \\
\hline Water Input & 2667.8 & 523.0 & 144.8 & $100 \%$ \\
\hline Evap & 148.0 & 147.8 & 0.2 & \\
\hline$\overline{\mathrm{G} 1-1}$ & 7.5 & 26.1 & 18.6 & \multirow[b]{5}{*}{$13.5 \%$} \\
\hline$\overline{\mathrm{G} 1-2}$ & 7.2 & 8.1 & 0.9 & \\
\hline$\overline{\mathrm{G} 1-3}$ & & & 0 & \\
\hline$\overline{\mathrm{G} 1-4}$ & & & 0 & \\
\hline SS-1 & & & 0 & \\
\hline G2-1 & & & 0 & \multirow[b]{5}{*}{$0.0 \%$} \\
\hline G2-2 & & & 0 & \\
\hline G2-3 & & & 0 & \\
\hline G2-4 & & & 0 & \\
\hline SS-2 & & & 0 & \\
\hline G3-1 & 7.4 & 25.4 & 18 & \multirow[b]{5}{*}{$36.1 \%$} \\
\hline G3-2 & 7.4 & 25.4 & 18 & \\
\hline G3-3 & 7.5 & 20.7 & 13.2 & \\
\hline G3-4 & & & 0 & \\
\hline SS-3 & 7.4 & 10.5 & 3.1 & \\
\hline G4-1 & & & 0 & \multirow[b]{5}{*}{$0.0 \%$} \\
\hline G4-2 & & & 0 & \\
\hline G4-3 & & & 0 & \\
\hline G4-4 & & & 0 & \\
\hline SS-4 & & & 0 & \\
\hline $\mathrm{B} 1$ & & & 0 & $0.0 \%$ \\
\hline $\mathrm{B} 2$ & & & 0 & $0.0 \%$ \\
\hline B3 & & & 0 & $0.0 \%$ \\
\hline B4 & & & 0 & $0.0 \%$ \\
\hline B5 & 109.2 & 112.2 & 3 & $2.1 \%$ \\
\hline B6 & 108.4 & 110.7 & 2.3 & $1.6 \%$ \\
\hline DS in-1 & & & 0 & \multirow[b]{2}{*}{$0.0 \%$} \\
\hline$\overline{D S}$ in-2 & & & 0 & \\
\hline DS out-1 & 7.4 & 43.9 & 36.5 & \multirow[b]{4}{*}{$36.2 \%$} \\
\hline DS out-2 & 7.4 & 18.6 & 11.2 & \\
\hline DS out-3 & 7.4 & 12.1 & 4.7 & \\
\hline DS out-4 & & & 0 & \\
\hline Other & & & 0 & $0.0 \%$ \\
\hline
\end{tabular}

Test Date: $\quad 5 / 22 / 02$

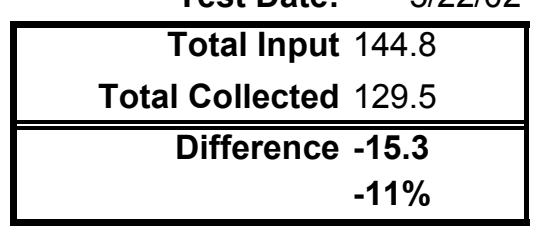

\begin{tabular}{|lr|}
\hline \multicolumn{2}{|c|}{ Evaporation } \\
\hline Evap Pan Tare Wt, g & 66.7 \\
Evaporation, g & 0.2 \\
\hline \% Evaporation & $\mathbf{0 . 2 5 \%}$ \\
\hline
\end{tabular}

\begin{tabular}{|lr|}
\hline \multicolumn{2}{|c|}{ Mass Flow Rate } \\
Test Duration, min & 30 \\
Flow Rate, g/min & 4.83 \\
\hline
\end{tabular}

\begin{tabular}{|lcccc|}
\hline \multicolumn{7}{l}{ Test Chamber Environment } & & & \\
\hline Initial Conditions & $\mathrm{RH}(\%)$ & Temperature $\left({ }^{\circ} \mathrm{C}\right)$ & Atm. P. (mbar) \\
1-HUM/TMP-BDST & 87.5 & 20.8 & P-IN-BDST & 875 \\
2-HUM/TMP-BDST & 86.8 & 20.7 & & \\
\hline Final Conditions & $\mathrm{RH}(\%)$ & Temperature $\left({ }^{\circ} \mathrm{C}\right)$ & & Atm. P. (mbar) \\
1-HUM/TMP-BDST & 91.9 & 20.8 & P-IN-BDST & 875 \\
2-HUM/TMP-BDST & 90 & 20.8 & & \\
\hline
\end{tabular}


Single Patch q(splash) Test Summary and Mass Balance-Smooth DS Surface

Test: Patch 5, $17.5 \mathrm{~cm}$ left of patch centerline, 33

Test Date: $\quad 5 / 17 / 02$

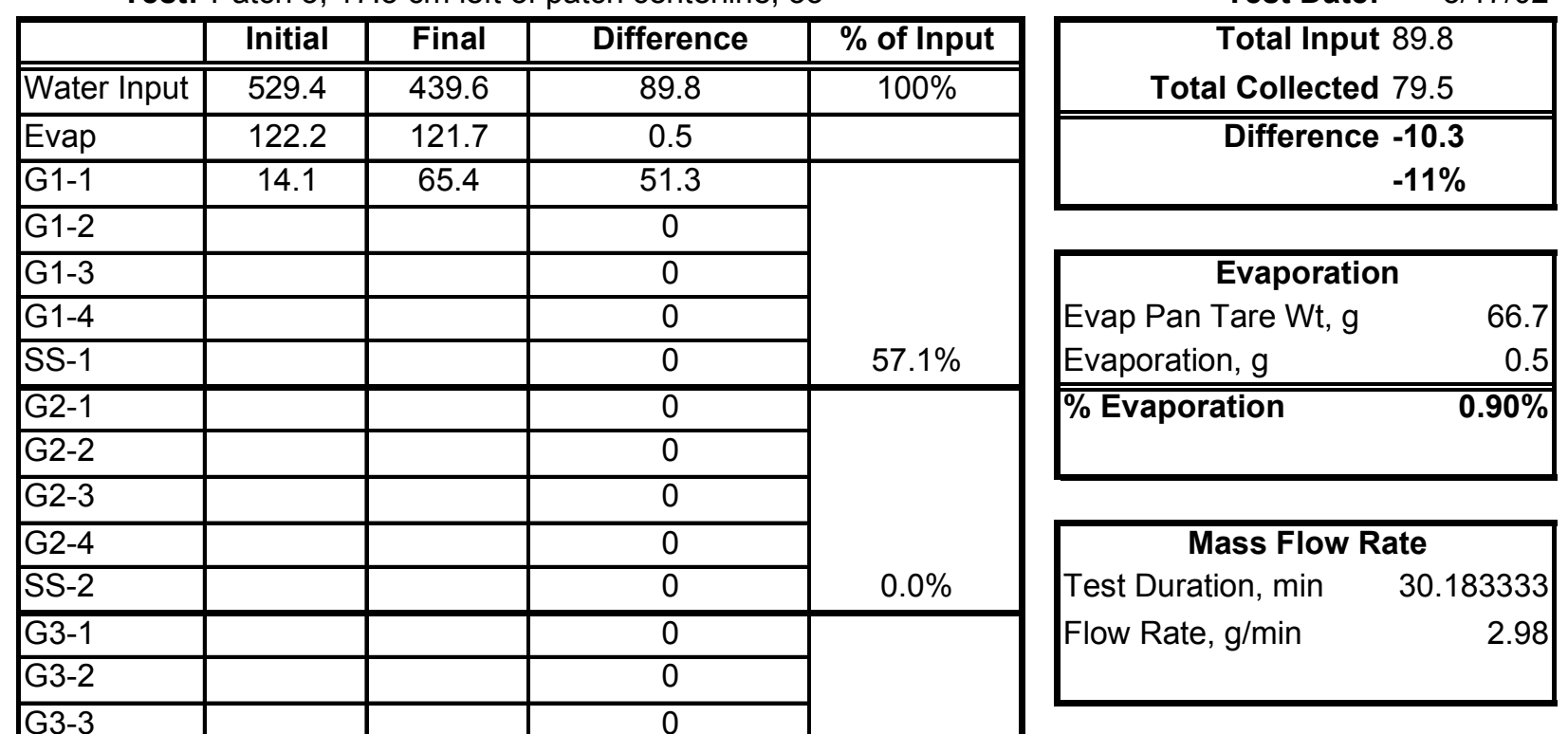

G3-4

SS-3

G4-1

G4-2

G4-3

G4-4

SS-4

$0.0 \%$

\begin{tabular}{|c|c|c|c|c|}
\hline B1 & & & 0 & $0.0 \%$ \\
\hline $\bar{B} 2$ & & & 0 & $0.0 \%$ \\
\hline B3 & & & 0 & $0.0 \%$ \\
\hline B4 & & & 0 & $0.0 \%$ \\
\hline B5 & 108.5 & 108.8 & 0.3 & $0.3 \%$ \\
\hline B6 & & & 0 & $0.0 \%$ \\
\hline$\overline{\mathrm{DS} \text { in-1 }}$ & & & 0 & \multirow[b]{2}{*}{$0.0 \%$} \\
\hline$\overline{\mathrm{DS}}$ in-2 & & & 0 & \\
\hline DS out-1 & $\overline{7.1}$ & 19.8 & 12.7 & \multirow[b]{4}{*}{$14.1 \%$} \\
\hline DS out- 2 & & & 0 & \\
\hline DS out-3 & & & 0 & \\
\hline DS out-4 & & & 0 & \\
\hline Other (floor) & 7.2 & 22.4 & 15.2 & $16.9 \%$ \\
\hline
\end{tabular}

Total Collected

79.5

$88.5 \%$

\begin{tabular}{|c|c|c|c|c|}
\hline \multicolumn{5}{|c|}{ Test Chamber Environment } \\
\hline Initial Conditions & $\mathrm{RH}(\%)$ & Temperature $\left({ }^{\circ} \mathrm{C}\right)$ & & Atm. P. (mbar) \\
\hline 1-HUM/TMP-BDST & 79.6 & 23.3 & P-IN-BDST & 871 \\
\hline 2-HUM/TMP-BDST & 80.1 & 22.7 & & \\
\hline Final Conditions & $\mathrm{RH}(\%)$ & Temperature $\left({ }^{\circ} \mathrm{C}\right)$ & & Atm. P. (mbar) \\
\hline 1-HUM/TMP-BDST & 84.6 & 23.4 & P-IN-BDST & 867 \\
\hline 2-HUM/TMP-BDST & 82.9 & 22.9 & & \\
\hline
\end{tabular}


Single Patch q(splash) Test Summary and Mass Balance-Smooth DS Surface

Test: Patch 6, Centerline, 16.5

\begin{tabular}{|c|c|c|c|c|}
\hline & Initial & Final & Difference & $\%$ of Input \\
\hline Water Input & "664.0 & $\overline{516.3}$ & 14147.7 & $10100 \%$ \\
\hline Evap & 156.8 & 156.3 & 0.5 & \\
\hline$\overline{G 1-1}$ & 7.4 & 52.8 & 45.4 & \multirow[b]{5}{*}{$85.8 \%$} \\
\hline$\overline{\mathrm{G} 1-2}$ & 7.4 & 46.3 & 38.9 & \\
\hline$\overline{\mathrm{G} 1-3}$ & 7.6 & 45.9 & 38.3 & \\
\hline G1-4 & 7.4 & 11.5 & 4.1 & \\
\hline SS-1 & & & 0 & \\
\hline$\overline{\mathrm{G} 2-1}$ & & & 0 & \multirow[b]{5}{*}{$0.0 \%$} \\
\hline G2-2 & & & 0 & \\
\hline $\bar{G} 2-3$ & & & 0 & \\
\hline G2-4 & & & 0 & \\
\hline SS-2 & & & 0 & \\
\hline G3-1 & $\overline{7.4}$ & $\overline{7.6}$ & 0.2 & \multirow[b]{5}{*}{$0.1 \%$} \\
\hline G3-2 & & & 0 & \\
\hline G3-3 & & & 0 & \\
\hline G3-4 & & & 0 & \\
\hline SS-3 & & & 0 & \\
\hline G4-1 & & & 0 & \multirow[b]{5}{*}{$0.0 \%$} \\
\hline$\overline{\mathrm{G} 4-2}$ & & & 0 & \\
\hline G4-3 & & & 0 & \\
\hline G4-4 & & & 0 & \\
\hline SS-4 & & & 0 & \\
\hline $\mathrm{B} 1$ & & & 0 & $0.0 \%$ \\
\hline B2 & & & 0 & $0.0 \%$ \\
\hline B3 & & & 0 & $0.0 \%$ \\
\hline B4 & 107.1 & 107.3 & 0.2 & $0.1 \%$ \\
\hline B5 & 109.2 & 109.4 & 0.2 & $0.1 \%$ \\
\hline B6 & 108.3 & 108.6 & 0.3 & $0.2 \%$ \\
\hline$\overline{D S}$ in-1 & & & 0 & \multirow[b]{2}{*}{$0.0 \%$} \\
\hline DS in-2 & & & 0 & \\
\hline DS out-1 & 7.4 & 16.1 & 8.7 & \multirow[b]{4}{*}{$6.9 \%$} \\
\hline DS out- 2 & 7.4 & 8.9 & 1.5 & \\
\hline DS out- 3 & & & 0 & \\
\hline DS out-4 & & & 0 & \\
\hline Other & & & 0 & $0.0 \%$ \\
\hline
\end{tabular}

Test Date 5/21/02

Total Input 147.7
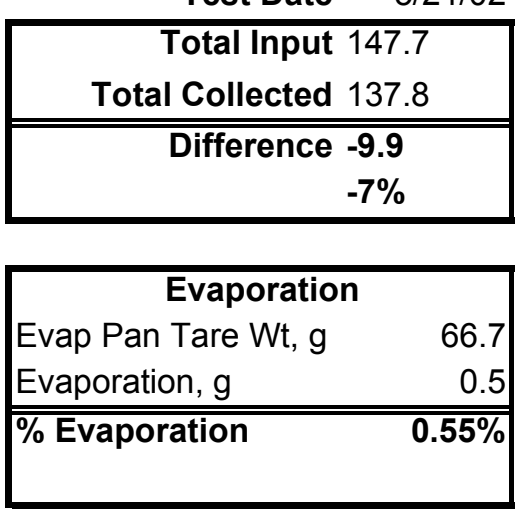

\begin{tabular}{|lr|}
\hline \multicolumn{2}{|c|}{ Mass Flow Rate } \\
Test Duration, min & 30 \\
Flow Rate, g/min & 4.92 \\
\hline
\end{tabular}

\begin{tabular}{|c|c|c|c|c|}
\hline \multicolumn{5}{|c|}{ Test Chamber Environment } \\
\hline Initial Conditions & $\mathrm{RH}(\%)$ & Temperature $\left({ }^{\circ} \mathrm{C}\right)$ & & Atm. P. (mbar) \\
\hline 1-HUM/TMP-BDST & 90.3 & 21.4 & P-IN-BDST & 884 \\
\hline 2-HUM/TMP-BDST & 87.6 & 21.5 & & \\
\hline Final Conditions & $\mathrm{RH}(\%)$ & Temperature $\left({ }^{\circ} \mathrm{C}\right)$ & & Atm. P. (mbar) \\
\hline 1-HUM/TMP-BDST & 90.6 & 21.4 & P-IN-BDST & 892 \\
\hline 2-HUM/TMP-BDST & 88.2 & 21.4 & & \\
\hline
\end{tabular}


Single Patch q(splash) Test Summary and Mass Balance-Smooth DS Surface

Test: Patch 6, Centerline, Between crown and 16.5

\begin{tabular}{|c|c|c|c|c|}
\hline & Initial & Final & Difference & $\%$ of Input \\
\hline Water Input & 667.8 & 525.6 & 142.2 & $100 \%$ \\
\hline Evap & 141.6 & 141.4 & 0.2 & \\
\hline G1-1 & 7.3 & 40.4 & 33.1 & \multirow[b]{5}{*}{$51.1 \%$} \\
\hline $\mathrm{G} 1-2$ & 7.4 & 45.9 & 38.5 & \\
\hline G1-3 & 7.3 & 8.2 & 0.9 & \\
\hline G1-4 & & & 0 & \\
\hline SS-1 & 7.2 & 7.4 & 0.2 & \\
\hline$\overline{\mathrm{G} 2-1}$ & & & 0 & \multirow[b]{5}{*}{$0.0 \%$} \\
\hline G2-2 & & & 0 & \\
\hline$\overline{\mathrm{G} 2-3}$ & & & 0 & \\
\hline G2-4 & & & 0 & \\
\hline SS-2 & & & 0 & \\
\hline G3-1 & 7.4 & 8.0 & 0.6 & \multirow[b]{5}{*}{$0.4 \%$} \\
\hline G3-2 & & & 0 & \\
\hline G3-3 & & & 0 & \\
\hline G3-4 & & & 0 & \\
\hline SS-3 & & & 0 & \\
\hline$\overline{\mathrm{G} 4-1}$ & & & 0 & \multirow[b]{5}{*}{$0.0 \%$} \\
\hline G4-2 & & & 0 & \\
\hline G4-3 & & & 0 & \\
\hline G4-4 & & & 0 & \\
\hline SS-4 & & & 0 & \\
\hline B1 & & & 0 & $0.0 \%$ \\
\hline B2 & & & 0 & $0.0 \%$ \\
\hline B3 & & & 0 & $0.0 \%$ \\
\hline B4 & 107.1 & 107.5 & 0.4 & $0.3 \%$ \\
\hline$\overline{B 5}$ & 109.0 & 109.7 & 0.7 & $0.5 \%$ \\
\hline B6 & 108.4 & 108.9 & 0.5 & $0.4 \%$ \\
\hline DS in-1 & & & 0 & \multirow[b]{2}{*}{$0.0 \%$} \\
\hline DS in-2 & & & 0 & \\
\hline$\overline{D S}$ out-1 & $\overline{7.2}$ & 34.3 & 27.1 & \multirow[b]{4}{*}{$33.5 \%$} \\
\hline DS out-2 & 7.4 & 12.4 & 5 & \\
\hline DS out-3 & 7.2 & 19.4 & 12.2 & \\
\hline DS out-4 & 7.3 & 10.6 & 3.3 & \\
\hline Other & & & 0 & $0.0 \%$ \\
\hline
\end{tabular}

Test Date $\quad 5 / 23 / 02$

\begin{tabular}{|r|}
\hline Total Input 142.2 \\
Total Collected 122.5 \\
\hline \hline Difference -19.7 \\
$-14 \%$ \\
\hline
\end{tabular}

\begin{tabular}{|lr|}
\hline \multicolumn{2}{|c|}{ Evaporation } \\
Evap Pan Tare Wt, g & 66.7 \\
Evaporation, g & 0.2 \\
\hline \% Evaporation & $\mathbf{0 . 2 7 \%}$ \\
\hline
\end{tabular}

\begin{tabular}{|lr|}
\hline \multicolumn{2}{|c|}{ Mass Flow Rate } \\
Test Duration, min & 30 \\
Flow Rate, g/min & 4.74 \\
\hline
\end{tabular}

\begin{tabular}{|lcccc|}
\hline Test Chamber Environment & & & \\
\hline Initial Conditions & $\mathrm{RH}(\%)$ & Temperature $\left({ }^{\circ} \mathrm{C}\right)$ & & Atm. P. (mbar) \\
1-HUM/TMP-BDST & 90.9 & 20 & P-IN-BDST & 880 \\
2-HUM/TMP-BDST & 88.4 & 19.8 & & \\
\hline Final Conditions & $\mathrm{RH}(\%)$ & Temperature $\left({ }^{\circ} \mathrm{C}\right)$ & & Atm. P. (mbar) \\
1-HUM/TMP-BDST & 91.2 & 20.4 & P-IN-BDST & 888 \\
2-HUM/TMP-BDST & 89.2 & 20.2 & & \\
\hline
\end{tabular}


Single Patch q(splash) Test Summary and Mass Balance-Smooth DS Surface

Test: Patch 6, $36.5 \mathrm{~cm}$ left of patch centerline, 16.5

\begin{tabular}{|c|c|c|c|c|}
\hline & Initial & Final & Difference & $\%$ of Input \\
\hline Water Input & 661.8 & 519.5 & 142.3 & $\overline{100 \%}$ \\
\hline Evap & 157.9 & 157.8 & 0.1 & \\
\hline$\overline{\mathrm{G} 1-1}$ & 7.4 & 57.6 & 50.2 & \multirow[b]{5}{*}{$67.2 \%$} \\
\hline$\overline{\mathrm{G} 1-2}$ & 7.4 & 48.8 & 41.4 & \\
\hline G1-3 & 7.4 & 10.1 & 2.7 & \\
\hline $\mathrm{G} 1-4$ & & & 0 & \\
\hline SS-1 & 7.3 & 8.6 & 1.3 & \\
\hline$\overline{\mathrm{G} 2-1}$ & & & 0 & \multirow[b]{5}{*}{$0.0 \%$} \\
\hline G2-2 & & & 0 & \\
\hline G2-3 & & & 0 & \\
\hline G2-4 & & & 0 & \\
\hline SS-2 & & & 0 & \\
\hline$\overline{\text { G3-1 }}$ & 7.4 & 7.8 & 0.4 & \multirow[b]{5}{*}{$0.3 \%$} \\
\hline G3-2 & & & 0 & \\
\hline G3-3 & & & 0 & \\
\hline G3-4 & & & 0 & \\
\hline SS-3 & & & 0 & \\
\hline$\overline{\mathrm{G}} 4-1$ & & & 0 & \multirow[b]{5}{*}{$0.0 \%$} \\
\hline G4-2 & & & 0 & \\
\hline G4-3 & & & 0 & \\
\hline G4-4 & & & 0 & \\
\hline SS-4 & & & 0 & \\
\hline $\mathrm{B} 1$ & & & 0 & $0.0 \%$ \\
\hline B2 & & & $\overline{0}$ & $0.0 \%$ \\
\hline B3 & & & 0 & $0.0 \%$ \\
\hline $\bar{B} 4$ & 107.3 & 108.9 & 1.6 & $1.1 \%$ \\
\hline B5 & 109.3 & 109.3 & 0 & $0.0 \%$ \\
\hline$\overline{B 6}$ & 108.6 & 108.7 & 0.1 & $0.1 \%$ \\
\hline$\overline{D S}$ in-1 & & & 0 & \multirow[b]{2}{*}{$0.0 \%$} \\
\hline DS in-2 & & & 0 & \\
\hline$\overline{D S}$ out-1 & 7.5 & 18.2 & 10.7 & \multirow[b]{4}{*}{$9.6 \%$} \\
\hline DS out-2 & 7.4 & 10.3 & 2.9 & \\
\hline DS out-3 & 7.3 & 7.4 & 0.1 & \\
\hline DS out-4 & & & 0 & \\
\hline Other & & & 0 & $0.0 \%$ \\
\hline
\end{tabular}

Test Date $\quad 5 / 21 / 02$

Total Input 142.3

Total Collected 111.4

Difference -30.9

$-22 \%$

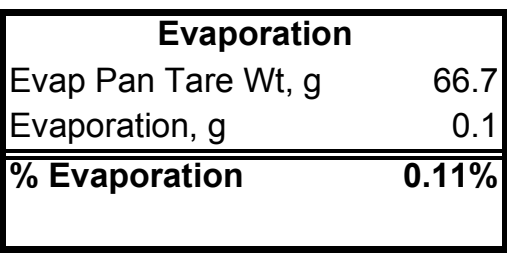

\begin{tabular}{|lr|}
\hline \multicolumn{2}{|c|}{ Mass Flow Rate } \\
Test Duration, min & 30 \\
Flow Rate, g/min & 4.74 \\
& \\
\hline
\end{tabular}

Test Chamber Environment

\begin{tabular}{|lcccc|}
\hline Initial Conditions & $\mathrm{RH}(\%)$ & Temperature $\left({ }^{\circ} \mathrm{C}\right)$ & & Atm. P. (mbar) \\
1-HUM/TMP-BDST & 91.7 & 22 & P-IN-BDST & 884 \\
2-HUM/TMP-BDST & 90.8 & 21.6 & & \\
\hline Final Conditions & $\mathrm{RH}(\%)$ & Temperature $\left({ }^{\circ} \mathrm{C}\right)$ & & Atm. P. (mbar) \\
1-HUM/TMP-BDST & 86.5 & 22.7 & P-IN-BDST & 886 \\
2-HUM/TMP-BDST & 80.5 & 22.8 & & \\
\hline
\end{tabular}


Single Patch q(splash) Test Summary and Mass Balance-Smooth DS Surface

Test: Patch 6, $36.5 \mathrm{~cm}$ left of patch centerline, Crown

\begin{tabular}{|c|c|c|c|c|}
\hline & Initial & Final & Difference & $\%$ of Input \\
\hline Water Input & 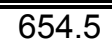 & $\overline{515.4}$ & "139.1 & "100\% \\
\hline Evap & 147.3 & 146.9 & 0.4 & \\
\hline $\mathrm{G} 1-1$ & 7.4 & 24.2 & 16.8 & \multirow[b]{5}{*}{$14.2 \%$} \\
\hline$\overline{\mathrm{G} 1-2}$ & 7.3 & 10.2 & 2.9 & \\
\hline G1-3 & & & 0 & \\
\hline $\mathrm{G} 1-4$ & & & 0 & \\
\hline SS-1 & & & 0 & \\
\hline G2-1 & & & 0 & \multirow[b]{5}{*}{$0.0 \%$} \\
\hline G2-2 & & & 0 & \\
\hline $\mathrm{G} 2-3$ & & & 0 & \\
\hline G2-4 & & & 0 & \\
\hline SS-2 & & & 0 & \\
\hline G3-1 & 7.4 & 37.8 & 30.4 & \multirow[b]{5}{*}{$39.6 \%$} \\
\hline G3-2 & 7.3 & 21.4 & 14.1 & \\
\hline G3-3 & 7.4 & 17.1 & 9.7 & \\
\hline G3-4 & & & 0 & \\
\hline SS-3 & 7.5 & 8.4 & 0.9 & \\
\hline$\overline{\mathrm{G} 4-1}$ & & & 0 & \multirow[b]{5}{*}{$0.0 \%$} \\
\hline G4-2 & & & 0 & \\
\hline G4-3 & & & 0 & \\
\hline G4-4 & & & 0 & \\
\hline SS-4 & & & 0 & \\
\hline $\mathrm{B} 1$ & & & 0 & $0.0 \%$ \\
\hline$\overline{B 2}$ & & & 0 & $0.0 \%$ \\
\hline B3 & & & 0 & $0.0 \%$ \\
\hline B4 & 107.2 & 108.0 & 0.8 & $0.6 \%$ \\
\hline B5 & & & 0 & $0.0 \%$ \\
\hline B6 & 108.4 & 111.3 & 2.9 & $2.1 \%$ \\
\hline DS in-1 & & & 0 & \multirow[b]{2}{*}{$0.0 \%$} \\
\hline DS in-2 & & & 0 & \\
\hline DS out-1 & 7.5 & 14.9 & 7.4 & \multirow[b]{4}{*}{$27.3 \%$} \\
\hline DS out- 2 & 7.6 & 8.4 & 0.8 & \\
\hline DS out-3 & 7.4 & 37.2 & 29.8 & \\
\hline DS out-4 & & & 0 & \\
\hline Other & & & 0 & $0.0 \%$ \\
\hline
\end{tabular}

Test Date $\quad 5 / 22 / 02$

Total Input 139.1

Total Collected 116.5

Difference -22.6

$-16 \%$

\begin{tabular}{|lr|}
\hline \multicolumn{2}{|c|}{ Evaporation } \\
Evap Pan Tare Wt, g & 66.7 \\
Evaporation, g & 0.4 \\
\hline \% Evaporation & $\mathbf{0 . 5 0 \%}$ \\
\hline
\end{tabular}

Mass Flow Rate

Test Duration, min $\quad 30.016667$

Flow Rate, $\mathrm{g} / \mathrm{min} \quad 4.63$

\begin{tabular}{|lcccc|}
\hline \multicolumn{7}{|l|}{ Test Chamber Environment } & & \\
\hline Initial Conditions & $\mathrm{RH}(\%)$ & Temperature $\left({ }^{\circ} \mathrm{C}\right)$ & & Atm. P. (mbar) \\
1-HUM/TMP-BDST & 89.4 & 21.6 & P-IN-BDST & 872 \\
2-HUM/TMP-BDST & 87.3 & 21.4 & & \\
\hline Final Conditions & $\mathrm{RH}(\%)$ & Temperature $\left({ }^{\circ} \mathrm{C}\right)$ & & Atm. P. (mbar) \\
1-HUM/TMP-BDST & 92.6 & 21.1 & P-IN-BDST & 877 \\
2-HUM/TMP-BDST & 90.8 & 21 & & \\
\hline
\end{tabular}


Single Patch q(splash) Test Summary and Mass Balance-Smooth DS Surface

Test: Patch 6, $36.5 \mathrm{~cm}$ left of cntrln, between Crown and 16.5

Test Date 5/23/02

\begin{tabular}{|c|c|c|c|c|c|}
\hline & Initial & Final & Difference & $\%$ of Input & \multirow{2}{*}{$\begin{array}{r}\text { Total Input } 129.3 \\
\text { Total Collected } 116.2\end{array}$} \\
\hline Water Input & 660.4 & 531.1 & 129.3 & \multirow[t]{2}{*}{$100 \%$} & \\
\hline Evap & 122.7 & 122.5 & 0.2 & & \multirow{2}{*}{$\begin{array}{r}\text { Difference }-13.1 \\
-10 \%\end{array}$} \\
\hline G1-1 & 7.3 & 53.9 & 46.6 & \multirow[b]{5}{*}{$57.0 \%$} & \\
\hline G1-2 & 7.3 & 23.2 & 15.9 & & \\
\hline G1-3 & $\overline{7.3}$ & 18.0 & 10.7 & & Evaporation \\
\hline G1-4 & 7.0 & 7.5 & 0.5 & & \multirow{2}{*}{$\begin{array}{l}\text { Evap Pan Tare Wt, g } \\
\text { Evaporation, g }\end{array}$} \\
\hline SS-1 & & & 0 & & \\
\hline $\mathrm{G} 2-1$ & & & 0 & \multirow[b]{5}{*}{$0.0 \%$} & \multirow[t]{2}{*}{ \% Evaporation } \\
\hline G2-2 & & & $\overline{0}$ & & \\
\hline G2-3 & & & 0 & & \multirow[b]{2}{*}{ Mass Flow Rate } \\
\hline G2-4 & & & 0 & & \\
\hline SS-2 & & & 0 & & \multirow{3}{*}{$\begin{array}{l}\text { Test Duration, min } \\
\text { Flow Rate, g/min }\end{array}$} \\
\hline G3-1 & 7.3 & 7.9 & 0.6 & & \\
\hline G3-2 & & & 0 & & \\
\hline G3-3 & & & 0 & & \\
\hline
\end{tabular}

\begin{tabular}{|c|c|c|c|c|}
\hline G3-4 & & & 0 & \\
\hline SS-3 & & & 0 & $0.5 \%$ \\
\hline$\overline{G 4-1}$ & & & 0 & \multirow[b]{5}{*}{$0.0 \%$} \\
\hline G4-2 & & & 0 & \\
\hline G4-3 & & & 0 & \\
\hline G4-4 & & & 0 & \\
\hline SS-4 & & & 0 & \\
\hline $\mathrm{B1}$ & & & 0 & $0.0 \%$ \\
\hline B2 & & & 0 & $0.0 \%$ \\
\hline$\overline{B 3}$ & & & 0 & $0.0 \%$ \\
\hline B4 & 107.0 & 113.6 & 6.6 & $5.1 \%$ \\
\hline B5 & & & 0 & $0.0 \%$ \\
\hline B6 & 108.3 & 108.8 & 0.5 & $0.4 \%$ \\
\hline DS in-1 & & & 0 & \multirow[b]{2}{*}{$0.0 \%$} \\
\hline DS in-2 & & & 0 & \\
\hline DS out-1 & 7.4 & 26.1 & 18.7 & \multirow[b]{4}{*}{$26.9 \%$} \\
\hline DS out-2 & 7.2 & 20.3 & 13.1 & \\
\hline DS out-3 & 7.2 & 10.2 & 3 & \\
\hline DS out-4 & & & 0 & \\
\hline Other & & & 0 & $0.0 \%$ \\
\hline
\end{tabular}

Test Chamber Environment

\begin{tabular}{|lcccc|}
\hline Initial Conditions & $\mathrm{RH}(\%)$ & Temperature $\left({ }^{\circ} \mathrm{C}\right)$ & & Atm. P. (mbar) \\
1-HUM/TMP-BDST & 86.2 & 19.1 & P-IN-BDST & 881 \\
2-HUM/TMP-BDST & 88.7 & 18.8 & & \\
\hline Final Conditions & $\mathrm{RH}(\%)$ & Temperature $\left({ }^{\circ} \mathrm{C}\right)$ & & Atm. P. (mbar) \\
1-HUM/TMP-BDST & 91.6 & 19.6 & P-IN-BDST & 878 \\
2-HUM/TMP-BDST & 89.2 & 19.5 & & \\
\hline
\end{tabular}


Single Patch q(film) Test Summary and Mass Balance - Smooth DS Surface

Test: Patch 2, $15 \mathrm{~cm}$ right of center, Crown

\begin{tabular}{|c|c|c|c|c|}
\hline & Initial & Final & Difference & $\%$ of Input \\
\hline Water Input & $\begin{array}{l}-23.6 \\
\end{array}$ & -137.7 & 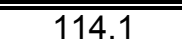 & $\overline{100 \%}$ \\
\hline Evap & 96.6 & 95.3 & 1.3 & \\
\hline$\overline{\mathrm{G} 1-1}$ & 7.4 & 28.0 & 20.6 & \multirow[b]{5}{*}{$20.9 \%$} \\
\hline$\overline{\mathrm{G} 1-2}$ & 7.6 & 10.8 & 3.2 & \\
\hline G1-3 & & & 0 & \\
\hline$\overline{G 1-4}$ & & & 0 & \\
\hline SS-1 & & & $\overline{0}$ & \\
\hline$\overline{\mathrm{G} 2-1}$ & & & $\overline{0}$ & \multirow[b]{5}{*}{$0.0 \%$} \\
\hline G2-2 & & & 0 & \\
\hline G2-3 & & & 0 & \\
\hline $\bar{G} 2-4$ & & & 0 & \\
\hline SS-2 & & & 0 & \\
\hline G3-1 & 7.3 & 14.4 & $\overline{7.1}$ & \multirow[b]{5}{*}{$11.0 \%$} \\
\hline G3-2 & 7.3 & 11.7 & 4.4 & \\
\hline G3-3 & & & 0 & \\
\hline G3-4 & & & 0 & \\
\hline SS-3 & 7.2 & 8.2 & 1 & \\
\hline$\overline{\mathrm{G}} 4-1$ & & & $\overline{0}$ & \multirow[b]{5}{*}{$0.0 \%$} \\
\hline G4-2 & & & 0 & \\
\hline G4-3 & & & 0 & \\
\hline G4-4 & & & 0 & \\
\hline SS-4 & & & 0 & \\
\hline$\overline{\mathrm{B} 1}$ & & & 0 & $0.0 \%$ \\
\hline B2 & 106.8 & 112.9 & 6.1 & $5.3 \%$ \\
\hline B3 & & & 0 & $0.0 \%$ \\
\hline B4 & & & 0 & $0.0 \%$ \\
\hline B5 & 109.6 & 109.5 & -0.1 & $-0.1 \%$ \\
\hline B6 & & & 0 & $0.0 \%$ \\
\hline$\overline{D S}$ in-1 & & & 0 & \multirow[b]{2}{*}{$0.0 \%$} \\
\hline DS in-2 & & & 0 & \\
\hline$\overline{D S}$ out-1 & 7.3 & 8.3 & 1 & \multirow[b]{4}{*}{$33.7 \%$} \\
\hline DS out- 2 & 7.4 & 14.5 & 7.1 & \\
\hline DS out-3 & 7.3 & 37.6 & 30.3 & \\
\hline DS out-4 & & & 0 & \\
\hline Other & & & 0 & $0.0 \%$ \\
\hline
\end{tabular}

Test Date $\quad 5 / 29 / 02$

Total Input 114.1

Total Collected 80.7

Difference -33.4 $-29 \%$

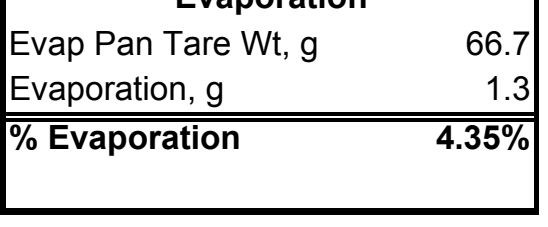

\begin{tabular}{|lr|}
\hline \multicolumn{2}{|c|}{ Mass Flow Rate } \\
Test Duration, min & 34.4 \\
Flow Rate, g/min & 3.32 \\
\hline
\end{tabular}

Test Chamber Environment

\begin{tabular}{|lcccc|}
\hline Initial Conditions & $\mathrm{RH}(\%)$ & Temperature $\left({ }^{\circ} \mathrm{C}\right)$ & & Atm. P. (mbar) \\
1-HUM/TMP-BDST & 87.8 & 25.7 & P-IN-BDST & 857 \\
2-HUM/TMP-BDST & 84.5 & 25.7 & & \\
\hline Final Conditions & $\mathrm{RH}(\%)$ & Temperature $\left({ }^{\circ} \mathrm{C}\right)$ & & Atm. P. (mbar) \\
1-HUM/TMP-BDST & 90.1 & 25.8 & P-IN-BDST & 870 \\
2-HUM/TMP-BDST & 85.9 & 26.3 & & \\
\hline
\end{tabular}


Single Patch q(film) Test Summary and Mass Balance - Smooth DS Surface

Test: Patch 2, Centerline, $10.5 \mathrm{~cm}$ forward from crown

\begin{tabular}{|c|c|c|c|c|}
\hline & Initial & Final & Difference & $\%$ of Input \\
\hline Water Input & 538.1 & 468.9 & 69.2 & $100 \%$ \\
\hline Evap & 90.3 & 89.3 & 1 & \\
\hline G1-1 & 7.4 & 13.7 & 6.3 & \multirow[b]{5}{*}{$9.8 \%$} \\
\hline G1-2 & 7.4 & 7.9 & 0.5 & \\
\hline G1-3 & & & 0 & \\
\hline G1-4 & & & 0 & \\
\hline SS-1 & & & 0 & \\
\hline$\overline{\mathrm{G} 2-1}$ & & & 0 & \multirow[b]{5}{*}{$0.0 \%$} \\
\hline G2-2 & & & 0 & \\
\hline $\bar{G} 2-3$ & & & 0 & \\
\hline G2-4 & & & 0 & \\
\hline SS-2 & & & 0 & \\
\hline G3-1 & 7.3 & 7.9 & 0.6 & \multirow[b]{5}{*}{$3.2 \%$} \\
\hline G3-2 & & & 0 & \\
\hline G3-3 & & & 0 & \\
\hline G3-4 & & & 0 & \\
\hline SS-3 & 7.3 & 8.9 & 1.6 & \\
\hline G4-1 & & & 0 & \multirow[b]{5}{*}{$0.0 \%$} \\
\hline G4-2 & & & 0 & \\
\hline G4-3 & & & 0 & \\
\hline G4-4 & & & 0 & \\
\hline SS-4 & & & 0 & \\
\hline B1 & & & 0 & $0.0 \%$ \\
\hline B2 & 106.5 & 117.2 & 10.7 & $15.5 \%$ \\
\hline B3 & & & 0 & $0.0 \%$ \\
\hline B4 & 107.5 & 107.6 & 0.1 & $0.1 \%$ \\
\hline B5 & 109.5 & 109.5 & 0 & $0.0 \%$ \\
\hline $\mathrm{B} 6$ & & & 0 & $0.0 \%$ \\
\hline DS in-1 & & & 0 & \multirow[b]{2}{*}{$0.0 \%$} \\
\hline DS in-2 & & & 0 & \\
\hline DS out-1 & 7.4 & 11.8 & 4.4 & \multirow[b]{4}{*}{$41.5 \%$} \\
\hline DS out-2 & 7.3 & 28.0 & 20.7 & \\
\hline DS out-3 & 7.5 & 11.1 & 3.6 & \\
\hline DS out-4 & & & 0 & \\
\hline Other G1-5 & & & 0 & $0.0 \%$ \\
\hline
\end{tabular}

Test Date $\quad 5 / 31 / 02$

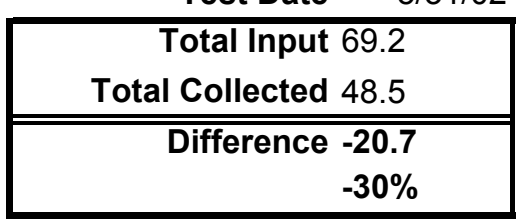

\begin{tabular}{|lr|}
\hline \multicolumn{2}{|c|}{ Evaporation } \\
Evap Pan Tare Wt, g & 66.7 \\
Evaporation, g & 1 \\
\hline \% Evaporation & $\mathbf{4 . 2 4 \%}$ \\
\hline
\end{tabular}

\begin{tabular}{|lr|}
\hline \multicolumn{2}{|c|}{ Mass Flow Rate } \\
Test Duration, min & 30.016667 \\
Flow Rate, g/min & 2.31 \\
& \\
\hline
\end{tabular}

Test Chamber Environment

\begin{tabular}{|lcccc|}
\hline Initial Conditions & $\mathrm{RH}(\%)$ & Temperature $\left({ }^{\circ} \mathrm{C}\right)$ & & Atm. P. (mbar) \\
1-HUM/TMP-BDST & 88 & 24.6 & P-IN-BDST & 865 \\
2-HUM/TMP-BDST & 84.9 & 24.7 & & \\
\hline Final Conditions & $\mathrm{RH}(\%)$ & Temperature $\left({ }^{\circ} \mathrm{C}\right)$ & & Atm. P. (mbar) \\
1-HUM/TMP-BDST & 89.7 & 25.3 & P-IN-BDST & 862 \\
2-HUM/TMP-BDST & 87.1 & 25.5 & & \\
\hline
\end{tabular}


Single Patch q(film) Test Summary and Mass Balance - Smooth DS Surface

Test: Patch 2, $15 \mathrm{~cm}$ right of center, 16.5

\begin{tabular}{|c|c|c|c|c|}
\hline & Initial & Final & Difference & $\%$ of Input \\
\hline Water Input & $\overline{36.1}$ & -141.1 & 177.2 & $100 \%$ \\
\hline$\overline{\text { Evap }}$ & 96.3 & 96.2 & 0.1 & \\
\hline G1-1 & 7.6 & 54.3 & 46.7 & \multirow[b]{5}{*}{$68.0 \%$} \\
\hline$\overline{\mathrm{G} 1-2}$ & 7.5 & 50.9 & 43.4 & \\
\hline G1-3 & $\overline{7.4}$ & 36.7 & 29.3 & \\
\hline$\overline{\mathrm{G} 1-4}$ & & & 0 & \\
\hline SS-1 & 7.5 & 8.6 & 1.1 & \\
\hline$\overline{\mathrm{G} 2-1}$ & & & 0 & \multirow[b]{5}{*}{$0.0 \%$} \\
\hline G2-2 & & & 0 & \\
\hline G2-3 & & & $\overline{0}$ & \\
\hline G2-4 & & & 0 & \\
\hline SS-2 & & & 0 & \\
\hline$\overline{\mathrm{G}} 3-1$ & & & 0 & \multirow[b]{5}{*}{$0.0 \%$} \\
\hline G3-2 & & & 0 & \\
\hline G3-3 & & & 0 & \\
\hline G3-4 & & & 0 & \\
\hline SS-3 & 7.6 & 7.6 & 0 & \\
\hline G4-1 & & & 0 & \multirow[b]{5}{*}{$0.0 \%$} \\
\hline $\mathrm{G} 4-2$ & & & 0 & \\
\hline G4-3 & & & 0 & \\
\hline G4-4 & & & 0 & \\
\hline SS-4 & & & 0 & \\
\hline$\overline{B 1}$ & & & 0 & $0.0 \%$ \\
\hline B2 & 107.1 & 112.0 & 4.9 & $2.8 \%$ \\
\hline B3 & & & 0 & $0.0 \%$ \\
\hline $\bar{B} 4$ & 107.2 & 107.5 & 0.3 & $0.2 \%$ \\
\hline B5 & 109.4 & 109.7 & 0.3 & $0.2 \%$ \\
\hline B6 & & & 0 & $0.0 \%$ \\
\hline$\overline{\mathrm{DS}}$ in-1 & & & 0 & \multirow[b]{2}{*}{$0.0 \%$} \\
\hline DS in-2 & & & 0 & \\
\hline DS out-1 & 7.5 & 7.9 & 0.4 & \multirow[b]{4}{*}{$11.1 \%$} \\
\hline DS out- 2 & 7.5 & 9.1 & 1.6 & \\
\hline DS out-3 & 7.6 & 17.4 & 9.8 & \\
\hline DS out-4 & 7.5 & 15.3 & 7.8 & \\
\hline Other & 7.5 & 8.8 & 1.3 & $0.7 \%$ \\
\hline
\end{tabular}

Test Date $\quad 5 / 21 / 02$

Total Input 177.2

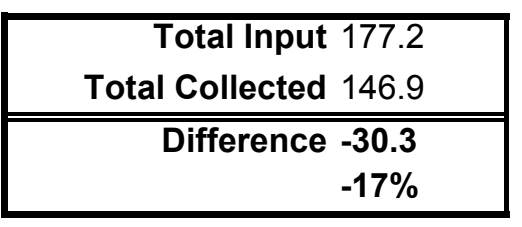

\begin{tabular}{|lr|}
\hline \multicolumn{2}{|c|}{ Evaporation } \\
Evap Pan Tare Wt, g & 66.7 \\
Evaporation, g & 0.1 \\
\hline \% Evaporation & $\mathbf{0 . 3 4 \%}$ \\
\hline
\end{tabular}

\begin{tabular}{|lr|}
\hline \multicolumn{2}{|c|}{ Mass Flow Rate } \\
Test Duration, min & 60.06667 \\
Flow Rate, g/min & 2.95 \\
\hline
\end{tabular}

Test Chamber Environment

\begin{tabular}{|lcccc|}
\hline Initial Conditions & $\mathrm{RH}(\%)$ & Temperature $\left({ }^{\circ} \mathrm{C}\right)$ & & Atm. P. (mbar) \\
1-HUM/TMP-BDST & 90.2 & 23 & P-IN-BDST & 863 \\
2-HUM/TMP-BDST & 89.6 & 27.7 & & \\
\hline Final Conditions & $\mathrm{RH}(\%)$ & Temperature $\left({ }^{\circ} \mathrm{C}\right)$ & & Atm. P. (mbar) \\
1-HUM/TMP-BDST & 93 & 24.3 & P-IN-BDST & 869 \\
2-HUM/TMP-BDST & 92.9 & 24.1 & & \\
\hline
\end{tabular}

SN-M\&O-SCl-043-V1, pages 128-129 
Single Patch q(film) Test Summary and Mass Balance - Smooth DS Surface

Test: Patch 2, centerline, 16.5

\begin{tabular}{|c|c|c|c|c|}
\hline & Initial & Final & Difference & $\%$ of Input \\
\hline Water Input & 28.7 & -213.7 & 242.4 & $100 \%$ \\
\hline Evap & 96.0 & 95.6 & 0.4 & \\
\hline G1-1 & 7.5 & 8.5 & 1 & \multirow[b]{5}{*}{$3.8 \%$} \\
\hline G1-2 & 7.5 & 11.5 & 4 & \\
\hline $\mathrm{G} 1-3$ & 7.6 & 11.9 & 4.3 & \\
\hline G1-4 & & & 0 & \\
\hline SS-1 & & & 0 & \\
\hline$\overline{\mathrm{G} 2-1}$ & & & 0 & \multirow[b]{5}{*}{$0.0 \%$} \\
\hline G2-2 & & & 0 & \\
\hline G2-3 & & & 0 & \\
\hline G2-4 & & & 0 & \\
\hline SS-2 & & & 0 & \\
\hline G3-1 & 7.6 & 7.6 & 0 & \multirow[b]{5}{*}{$0.0 \%$} \\
\hline G3-2 & & & 0 & \\
\hline G3-3 & & & 0 & \\
\hline G3-4 & & & 0 & \\
\hline SS-3 & & & 0 & \\
\hline G4-1 & & & 0 & \multirow[b]{5}{*}{$0.0 \%$} \\
\hline G4-2 & & & 0 & \\
\hline G4-3 & & & 0 & \\
\hline G4-4 & & & 0 & \\
\hline SS-4 & & & 0 & \\
\hline $\mathrm{B} 1$ & & & 0 & $0.0 \%$ \\
\hline B2 & 107.1 & 107.5 & 0.4 & $0.2 \%$ \\
\hline B3 & & & 0 & $0.0 \%$ \\
\hline B4 & 107.4 & 259.1 & 151.7 & $62.6 \%$ \\
\hline B5 & 109.6 & 109.7 & 0.1 & $0.0 \%$ \\
\hline B6 & & & 0 & $0.0 \%$ \\
\hline DS in-1 & & & 0 & \multirow[b]{2}{*}{$0.0 \%$} \\
\hline DS in-2 & & & 0 & \\
\hline DS out-1 & 7.5 & 8.8 & 1.3 & \multirow[b]{4}{*}{$11.4 \%$} \\
\hline DS out-2 & 7.5 & 19.5 & 12 & \\
\hline DS out-3 & 7.6 & 18.0 & 10.4 & \\
\hline DS out-4 & 7.5 & 11.4 & 3.9 & \\
\hline Other floor & 7.4 & 8.7 & 1.3 & $0.5 \%$ \\
\hline
\end{tabular}

Test Date $\quad 5 / 31 / 02$

\begin{tabular}{|r|}
\hline Total Input 242.4 \\
Total Collected 190.4 \\
\hline \hline Difference $\mathbf{- 5 2}$ \\
$\mathbf{- 2 1 \%}$ \\
\hline
\end{tabular}

\begin{tabular}{|lr|}
\hline \multicolumn{2}{|c|}{ Evaporation } \\
Evap Pan Tare Wt, g & 66.7 \\
Evaporation, g & 0.4 \\
\hline \% Evaporation & $\mathbf{1 . 3 7 \%}$ \\
\hline
\end{tabular}

\begin{tabular}{|lr|}
\hline \multicolumn{2}{|c|}{ Mass Flow Rate } \\
Test Duration, min & 66.15 \\
Flow Rate, g/min & 3.66 \\
\hline
\end{tabular}

\begin{tabular}{|c|c|c|c|c|}
\hline \multicolumn{5}{|c|}{ Test Chamber Environment } \\
\hline Initial Conditions & $\mathrm{RH}(\%)$ & Temperature $\left({ }^{\circ} \mathrm{C}\right)$ & & Atm. P. (mbar) \\
\hline 1-HUM/TMP-BDST & 92.1 & 24.6 & P-IN-BDST & 865 \\
\hline 2-HUM/TMP-BDST & 92.2 & 24.3 & & \\
\hline Final Conditions & $\mathrm{RH}(\%)$ & Temperature $\left({ }^{\circ} \mathrm{C}\right)$ & & Atm. P. (mbar) \\
\hline 1-HUM/TMP-BDST & 92.3 & 25.3 & P-IN-BDST & 865 \\
\hline 2-HUM/TMP-BDST & 89.1 & 25 & & \\
\hline
\end{tabular}

SN-M\&O-SCI-043-V1, pages 126-127 
Single Patch q(film) Test Summary and Mass Balance - Smooth DS Surface

Test: Patch 2, $15 \mathrm{~cm}$ right of center, 33

\begin{tabular}{|c|c|c|c|c|}
\hline & Initial & Final & Difference & $\%$ of Input \\
\hline Water Input & $\bar{~} 51.9$ & -84.8 & $\overline{136.7}$ & 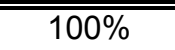 \\
\hline Evap & 92.4 & 91.1 & 1.3 & \\
\hline G1-1 & 7.6 & 61.2 & 53.6 & \multirow[b]{5}{*}{$63.9 \%$} \\
\hline $\mathrm{G} 1-2$ & 6.7 & 37.0 & 30.3 & \\
\hline G1-3 & 7.4 & 9.7 & 2.3 & \\
\hline $\mathrm{G} 1-4$ & 7.4 & 8.5 & 1.1 & \\
\hline SS-1 & & & 0 & \\
\hline $\bar{G} 2-1$ & & & 0 & \multirow[b]{5}{*}{$0.0 \%$} \\
\hline $\mathrm{G} 2-2$ & & & 0 & \\
\hline G2-3 & & & 0 & \\
\hline G2-4 & & & 0 & \\
\hline SS-2 & & & 0 & \\
\hline$\overline{G 3-1}$ & 7.3 & 7.9 & 0.6 & \multirow[b]{5}{*}{$0.4 \%$} \\
\hline G3-2 & & & 0 & \\
\hline G3-3 & & & 0 & \\
\hline G3-4 & & & 0 & \\
\hline SS-3 & & & 0 & \\
\hline$\overline{\mathrm{G} 4-1}$ & & & $\overline{0}$ & \multirow[b]{5}{*}{$0.0 \%$} \\
\hline G4-2 & & & 0 & \\
\hline G4-3 & & & 0 & \\
\hline G4-4 & & & 0 & \\
\hline SS-4 & & & 0 & \\
\hline$\overline{\mathrm{B} 1}$ & & & 0 & $0.0 \%$ \\
\hline B2 & 114.7 & 137.2 & 22.5 & $16.5 \%$ \\
\hline B3 & & & 0 & $0.0 \%$ \\
\hline B4 & & & 0 & $0.0 \%$ \\
\hline B5 & 109.4 & 109.5 & 0.1 & $0.1 \%$ \\
\hline $\mathrm{B} 6$ & & & 0 & $0.0 \%$ \\
\hline$\overline{D S}$ in-1 & 7.4 & 7.9 & 0.5 & \multirow[b]{2}{*}{$0.4 \%$} \\
\hline DS in-2 & & & 0 & \\
\hline$\overline{D S}$ out-1 & $\overline{7.4}$ & 10.5 & 3.1 & \multirow[b]{4}{*}{$2.5 \%$} \\
\hline DS out-2 & 7.4 & 7.7 & 0.3 & \\
\hline DS out-3 & & & 0 & \\
\hline DS out-4 & & & 0 & \\
\hline Other (floor) & 7.2 & 9.7 & 2.5 & $1.8 \%$ \\
\hline
\end{tabular}

Test Date $\quad 5 / 31 / 02$

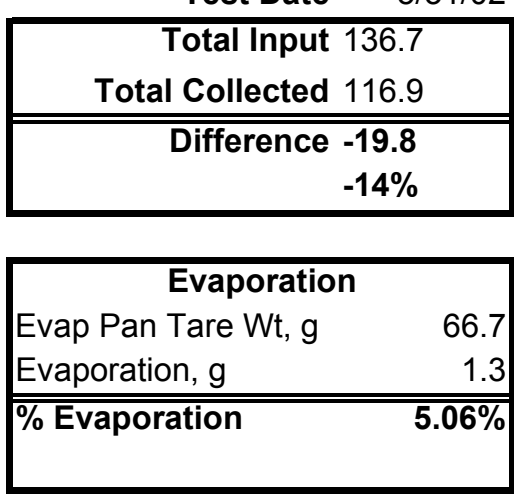

\begin{tabular}{|lr|}
\hline \multicolumn{2}{|c|}{ Mass Flow Rate } \\
Test Duration, min & 30.05 \\
Flow Rate, g/min & 4.55 \\
& \\
\hline
\end{tabular}

\begin{tabular}{|lcccc|}
\hline Test Chamber Environment & & & \\
\hline Initial Conditions & $\mathrm{RH}(\%)$ & Temperature $\left({ }^{\circ} \mathrm{C}\right)$ & & Atm. P. (mbar) \\
1-HUM/TMP-BDST & 88.3 & 26.1 & P-IN-BDST & 865 \\
2-HUM/TMP-BDST & 91.3 & 25.5 & & \\
\hline Final Conditions & $\mathrm{RH}(\%)$ & Temperature $\left({ }^{\circ} \mathrm{C}\right)$ & & Atm. P. (mbar) \\
1-HUM/TMP-BDST & 89.8 & 26.2 & P-IN-BDST & 865 \\
2-HUM/TMP-BDST & 93.3 & 25.7 & & \\
\hline
\end{tabular}


Single Patch q(film) Test Summary and Mass Balance - Smooth DS Surface

Test: Patch 2, Centerline, 33

\begin{tabular}{|c|c|c|c|c|}
\hline & Initial & Final & Difference & $\%$ of Input \\
\hline Water Input & $\overline{97.3}$ & -106.6 & 203.9 & $\overline{100 \%}$ \\
\hline Evap & 92.1 & 92.6 & -0.5 & \\
\hline$\overline{\mathrm{G} 1-1}$ & 7.5 & 11.6 & 4.1 & \multirow[b]{5}{*}{$2.5 \%$} \\
\hline$\overline{\mathrm{G} 1-2}$ & 7.6 & 8.0 & 0.4 & \\
\hline G1-3 & 7.6 & 8.1 & 0.5 & \\
\hline$\overline{\mathrm{G} 1-4}$ & & & 0 & \\
\hline SS-1 & & & 0 & \\
\hline G2-1 & & & 0 & \multirow[b]{5}{*}{$0.0 \%$} \\
\hline $\mathrm{G} 2-2$ & & & 0 & \\
\hline $\mathrm{G} 2-3$ & & & 0 & \\
\hline G2-4 & & & 0 & \\
\hline SS-2 & & & 0 & \\
\hline G3-1 & & & 0 & \multirow[b]{5}{*}{$0.0 \%$} \\
\hline G3-2 & & & 0 & \\
\hline G3-3 & & & 0 & \\
\hline G3-4 & & & 0 & \\
\hline SS-3 & & & 0 & \\
\hline G4-1 & & & 0 & \multirow[b]{5}{*}{$0.0 \%$} \\
\hline G4-2 & & & 0 & \\
\hline G4-3 & & & 0 & \\
\hline G4-4 & & & 0 & \\
\hline SS-4 & & & 0 & \\
\hline $\mathrm{B} 1$ & & & 0 & $0.0 \%$ \\
\hline $\mathrm{B} 2$ & 114.4 & 278.2 & 163.8 & $80.3 \%$ \\
\hline B3 & & & 0 & $0.0 \%$ \\
\hline $\bar{B} 4$ & & & 0 & $0.0 \%$ \\
\hline B5 & & & 0 & $0.0 \%$ \\
\hline $\mathrm{B} 6$ & & & 0 & $0.0 \%$ \\
\hline$\overline{D S}$ in-1 & & & 0 & \multirow[b]{2}{*}{$0.0 \%$} \\
\hline$\overline{D S}$ in-2 & & & 0 & \\
\hline DS out-1 & 7.5 & 7.8 & 0.3 & \multirow[b]{4}{*}{$1.3 \%$} \\
\hline DS out-2 & 7.7 & 7.7 & 0 & \\
\hline DS out-3 & 7.5 & 9.8 & 2.3 & \\
\hline DS out-4 & & & 0 & \\
\hline Other & & & 0 & $0.0 \%$ \\
\hline
\end{tabular}

Test Date 5/31/02

Total Input 203.9

\begin{tabular}{|r|}
\hline Total Input 203.9 \\
Total Collected 171.4 \\
\hline \hline Difference -32.5 \\
$-16 \%$ \\
\hline
\end{tabular}

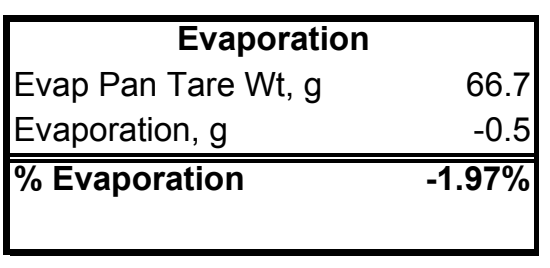

\begin{tabular}{|lr|}
\hline \multicolumn{2}{|c|}{ Mass Flow Rate } \\
Test Duration, $\min$ & 42.016667 \\
Flow Rate, g/min & 4.85 \\
\hline
\end{tabular}

\begin{tabular}{|lcccc|}
\hline \multicolumn{7}{l}{ Test Chamber Environment } & \multicolumn{3}{l|}{} \\
\hline Initial Conditions & $\mathrm{RH}(\%)$ & Temperature $\left({ }^{\circ} \mathrm{C}\right)$ & & Atm. P. (mbar) \\
1-HUM/TMP-BDST & 84.4 & 25.3 & P-IN-BDST & 866 \\
2-HUM/TMP-BDST & 83 & 25.1 & & \\
\hline Final Conditions & $\mathrm{RH}(\%)$ & Temperature $\left({ }^{\circ} \mathrm{C}\right)$ & & Atm. P. (mbar) \\
1-HUM/TMP-BDST & 88.8 & 25.9 & P-IN-BDST & 863 \\
2-HUM/TMP-BDST & 92.2 & 25.3 & & \\
\hline
\end{tabular}


Single Patch q(film) Test Summary and Mass Balance - Smooth DS Surface

Test: Patch 4, $8 \mathrm{~cm}$ right of centerline, 33

Test Date: $\quad 6 / 1 / 02$

\begin{tabular}{|l|c|c|c|c|}
\hline & Initial (g) & Final (g) & Difference (g) & \% of Input \\
\hline \hline Water Input & 53.7 & -83.7 & 137.4 & $100 \%$ \\
\hline Evap & 106.7 & 106.4 & 0.3 & \\
\hline G1-1 & 7.4 & 13.0 & 5.6 &
\end{tabular}

\begin{tabular}{|r|}
\hline Total Input (g) 137.4 \\
Total Collected (g) 118.9 \\
\hline \hline Difference (g) -18.5 \\
$-13 \%$ \\
\hline
\end{tabular}

\begin{tabular}{|l|l|l|l|}
\hline $\mathrm{G} 1-2$ & 7.2 & 8.6 & 1.4 \\
\hline $\mathrm{G} 1-3$ & 7.4 & 7.7 & 0.3 \\
\hline
\end{tabular}

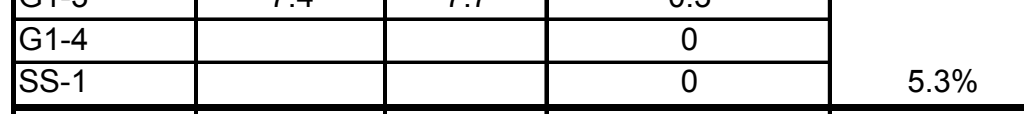

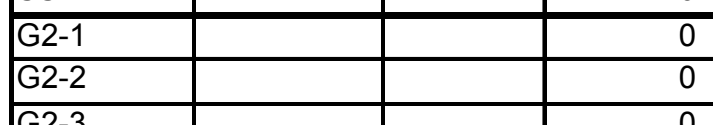

\begin{tabular}{|l|l|l|l|}
\hline $\mathrm{G} 2-3$ & & & 0 \\
\hline $\mathrm{G} 2-4$ & & 0 \\
\hline
\end{tabular}

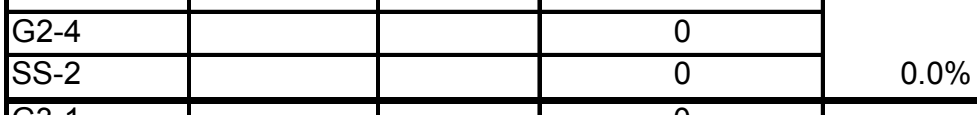

G3-1

G3-2

G3-3

G3-4

G4-1

G4-2

G4-3

G4-4

\begin{tabular}{|c|c|c|c|c|}
\hline SS-4 & & & 0 & $0.0 \%$ \\
\hline B1 & & & 0 & $0.0 \%$ \\
\hline B2 & 106.7 & 107.0 & 0.3 & $0.2 \%$ \\
\hline B3 & & & 0 & $0.0 \%$ \\
\hline B4 & 114.8 & 222.2 & 107.4 & $78.2 \%$ \\
\hline B5 & & & 0 & $0.0 \%$ \\
\hline B6 & & & 0 & $0.0 \%$ \\
\hline DS in-1 & 7.4 & 7.9 & 0.5 & \\
\hline DS in-2 & & & 0 & $0.4 \%$ \\
\hline DS out-1 & 7.3 & 10.3 & 3 & \\
\hline DS out-2 & 7.3 & 7.7 & 0.4 & \\
\hline DS out-3 & & & 0 & \\
\hline DS out-4 & & & 0 & $2.5 \%$ \\
\hline Other & & & 0 & $0.0 \%$ \\
\hline
\end{tabular}

\begin{tabular}{|lr|}
\hline Evap Pan Tare Wt $(\mathrm{g})$ & 67.7 \\
Evaporation $(\mathrm{g})$ & 0.3 \\
\hline \% Evaporation & $\mathbf{0 . 7 7 \%}$ \\
\hline
\end{tabular}

\begin{tabular}{|c|c|c|c|c|}
\hline \multicolumn{5}{|c|}{ Test Chamber Environment } \\
\hline Initial Conditions & $\mathrm{RH}(\%)$ & Temperature $\left({ }^{\circ} \mathrm{C}\right)$ & & Atm. P. (mbar) \\
\hline 1-HUM/TMP-BDST & 85 & 23.1 & P-IN-BDST & 869 \\
\hline 2-HUM/TMP-BDST & 88.4 & 22.7 & & \\
\hline Final Conditions & $\mathrm{RH}(\%)$ & Temperature $\left({ }^{\circ} \mathrm{C}\right)$ & & Atm. P. (mbar) \\
\hline 1-HUM/TMP-BDST & 90.4 & 23.7 & P-IN-BDST & 871 \\
\hline 2-HUM/TMP-BDST & 93.1 & 23.3 & & \\
\hline
\end{tabular}


Single Patch q(film) Test Summary and Mass Balance - Smooth DS Surface

Test: Patch $4,8 \mathrm{~cm}$ right of centerline, Crown

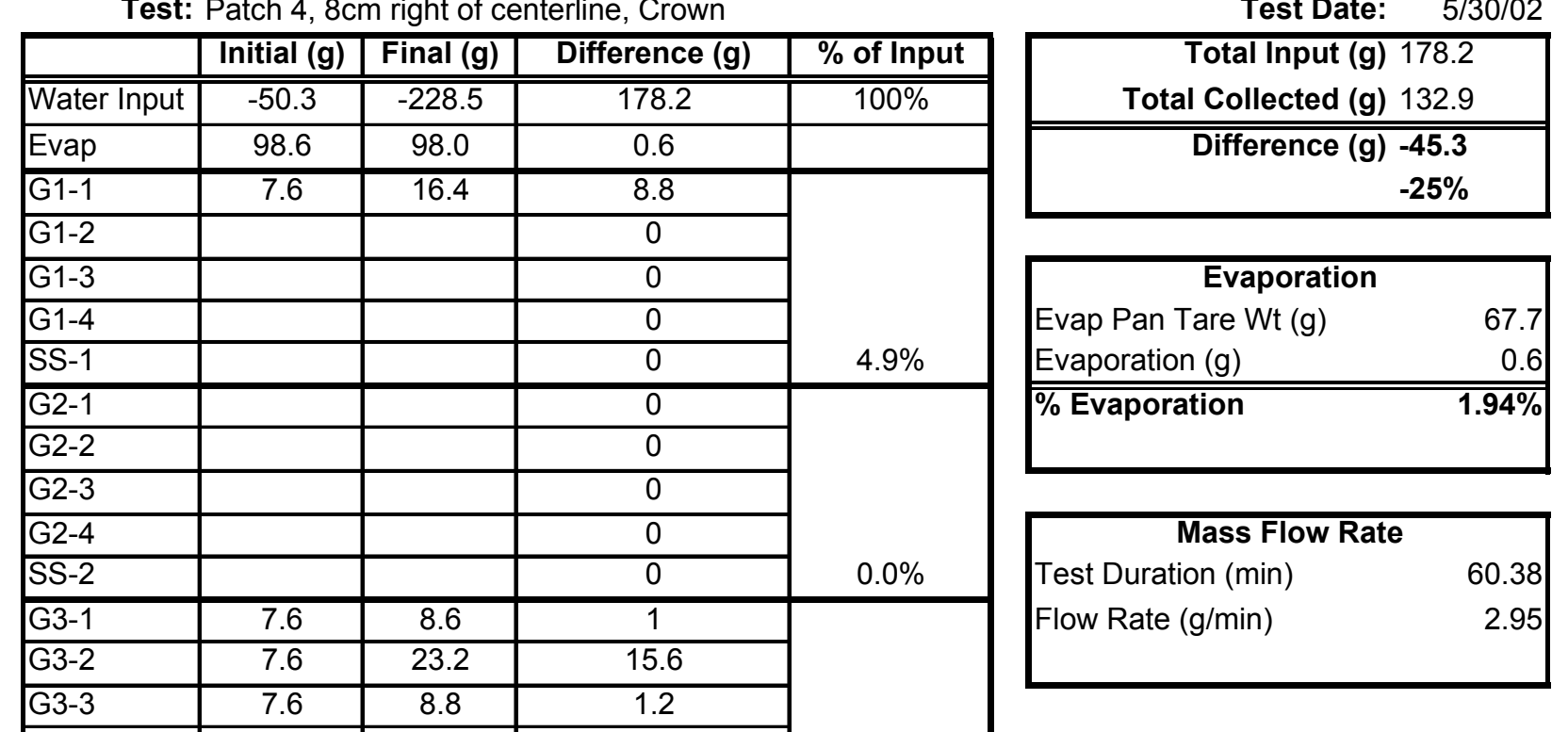

\begin{tabular}{|c|c|c|c|c|}
\hline G3-4 & 7.5 & 17.1 & 9.6 & \\
\hline SS-3 & & & 0 & $15.4 \%$ \\
\hline G4-1 & & & 0 & \multirow[b]{5}{*}{$0.0 \%$} \\
\hline G4-2 & & & 0 & \\
\hline G4-3 & & & 0 & \\
\hline G4-4 & & & 0 & \\
\hline SS-4 & & & 0 & \\
\hline B1 & & & 0 & $0.0 \%$ \\
\hline B2 & 107.0 & 109.0 & 2 & $1.1 \%$ \\
\hline B3 & & & 0 & $0.0 \%$ \\
\hline B4 & 107.6 & 129.6 & 22 & $12.3 \%$ \\
\hline B5 & & & 0 & $0.0 \%$ \\
\hline $\mathrm{B} 6$ & & & 0 & $0.0 \%$ \\
\hline DS in-1 & & & 0 & \multirow[b]{2}{*}{$0.0 \%$} \\
\hline DS in-2 & & & 0 & \\
\hline DS out-1 & 7.6 & 8.7 & 1.1 & \multirow[b]{4}{*}{$40.8 \%$} \\
\hline DS out-2 & 7.5 & 19.6 & 12.1 & \\
\hline DS out-3 & 7.5 & 34.4 & 26.9 & \\
\hline DS out-4 & 7.7 & 40.3 & 32.6 & \\
\hline Other & & & 0 & $0.0 \%$ \\
\hline
\end{tabular}

\begin{tabular}{|lcccc|}
\hline \multicolumn{4}{l}{ Test Chamber Environment } \\
\hline Initial Conditions & $\mathrm{RH}(\%)$ & Temperature $\left({ }^{\circ} \mathrm{C}\right)$ & Atm. P. (mbar) \\
1-HUM/TMP-BDST & 90.4 & 25.6 & P-IN-BDST & 854 \\
2-HUM/TMP-BDST & 83.8 & 25.7 & & \\
\hline Final Conditions & $\mathrm{RH}(\%)$ & Temperature $\left({ }^{\circ} \mathrm{C}\right)$ & Atm. P. (mbar) \\
1-HUM/TMP-BDST & 93.9 & 26.5 & P-IN-BDST & 865 \\
2-HUM/TMP-BDST & 91.3 & 26.2 & & \\
\hline
\end{tabular}


Single Patch q(film) Test Summary and Mass Balance - Smooth DS Surface

Test: Patch 4, $8 \mathrm{~cm}$ right of centerline, 16.5

\begin{tabular}{|c|c|c|c|c|c|}
\hline & Initial (g) & Final (g) & Difference (g) & $\%$ of Input & \multirow{2}{*}{$\begin{array}{r}\text { Total Input (g) } 172.3 \\
\text { Total Collected (g) } 120.1\end{array}$} \\
\hline Water Input & -0.9 & -173.2 & 172.3 & $100 \%$ & \\
\hline Evap & 96.7 & 96.4 & 0.3 & & \multirow{2}{*}{ 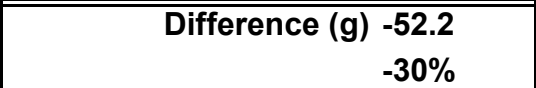 } \\
\hline G1-1 & 7.6 & 9.5 & 1.9 & \multirow[b]{5}{*}{$1.8 \%$} & \\
\hline G1-2 & & & 0 & & \\
\hline G1-3 & & & 0 & & Evaporation \\
\hline G1-4 & & & 0 & & Evap Pan Tare Wt (g) \\
\hline SS-1 & 7.5 & 8.7 & 1.2 & & Evaporation (g) \\
\hline G2-1 & & & 0 & & \% Evaporation \\
\hline G2-2 & & & 0 & & \\
\hline G2-3 & & & 0 & & \\
\hline G2-4 & & & 0 & & Mass Flow Rate \\
\hline SS-2 & 7.6 & 7.6 & 0 & $0.0 \%$ & Test Duration (min) \\
\hline G3-1 & & & 0 & & Flow Rate (g/min) \\
\hline G3-2 & & & 0 & & \\
\hline G3-3 & & & 0 & & \\
\hline
\end{tabular}

G3-4

SS-3

0

G4-1

G4-2

G4-3

G4-4

SS-4

B

$\mathrm{B2}$

\begin{tabular}{|c|c|c|c|c|}
\hline B2 & 106.9 & 107.9 & 1 & $0.6 \%$ \\
\hline B3 & & & 0 & $0.0 \%$ \\
\hline B4 & 107.1 & 199.6 & 92.5 & $53.7 \%$ \\
\hline B5 & & & 0 & $0.0 \%$ \\
\hline B6 & & & 0 & $0.0 \%$ \\
\hline DS in-1 & & & 0 & \multirow[b]{2}{*}{$0.0 \%$} \\
\hline DS in-2 & & & 0 & \\
\hline DS out-1 & 7.5 & 22.3 & 14.8 & \multirow[b]{4}{*}{$13.6 \%$} \\
\hline DS out- 2 & 7.6 & 14.4 & 6.8 & \\
\hline DS out- 3 & 7.4 & 9.3 & 1.9 & \\
\hline DS out-4 & & & 0 & \\
\hline Other & & & 0 & $0.0 \%$ \\
\hline
\end{tabular}

\begin{tabular}{|lcccc|}
\hline \multicolumn{5}{l}{ Test Chamber Environment } \\
\hline Initial Conditions & $\mathrm{RH}(\%)$ & Temperature $\left({ }^{\circ} \mathrm{C}\right)$ & Atm. P. (mbar) \\
1-HUM/TMP-BDST & 86.1 & 21.3 & P-IN-BDST & 873 \\
2-HUM/TMP-BDST & 87.5 & 21 & & \\
\hline Final Conditions & $\mathrm{RH}(\%)$ & Temperature $\left({ }^{\circ} \mathrm{C}\right)$ & Atm. P. (mbar) \\
1-HUM/TMP-BDST & 91.3 & 22.6 & P-IN-BDST & 871 \\
2-HUM/TMP-BDST & 92.1 & 22.2 & & \\
\hline
\end{tabular}


Single Patch q(film) Test Summary and Mass Balance - Smooth DS Surface

Test: Patch 5, Centerline, Crown

Test Date: $\quad 5 / 30 / 02$

\begin{tabular}{|c|c|c|c|c|c|c|}
\hline & Initial (g) & Final (g) & Difference $(\mathrm{g})$ & $\%$ of Input & \multirow{2}{*}{\multicolumn{2}{|c|}{$\begin{aligned} & \text { Total Input (g) } 163.8 \\
& \text { Total Collected }(\mathrm{g}) 141 \\
&\end{aligned}$}} \\
\hline Water Input & -12.6 & -176.4 & 163.8 & $10100 \%$ & & \\
\hline Evap & 77.0 & 76.5 & 0.5 & & \multirow{2}{*}{\multicolumn{2}{|c|}{$\begin{array}{r}\text { Difference }(\mathrm{g})-22.8 \\
-14 \%\end{array}$}} \\
\hline G1-1 & 7.5 & 8.2 & 0.7 & \multirow[b]{5}{*}{$0.4 \%$} & & \\
\hline G1-2 & & & 0 & & & \\
\hline G1-3 & & & 0 & & \multicolumn{2}{|c|}{ Evaporation } \\
\hline $\mathrm{G} 1-4$ & & & 0 & & \multirow{2}{*}{$\begin{array}{l}\text { Evap Pan Tare Wt (g) } \\
\text { Evaporation (g) }\end{array}$} & \multirow{2}{*}{$\begin{array}{r}67.7 \\
0.5\end{array}$} \\
\hline SS-1 & & & 0 & & & \\
\hline$\overline{\mathrm{G} 2-1}$ & & & 0 & \multirow[b]{5}{*}{$0.0 \%$} & \multirow[t]{2}{*}{ \% Evaporation } & \multirow[t]{2}{*}{$\overline{5.38 \%}$} \\
\hline G2-2 & & & 0 & & & \\
\hline G2-3 & & & 0 & & & \\
\hline G2-4 & & & 0 & & \multicolumn{2}{|c|}{ Mass Flow Rate } \\
\hline SS-2 & & & 0 & & \multirow{3}{*}{$\begin{array}{l}\text { Test Duration (min) } \\
\text { Flow Rate }(\mathrm{g} / \mathrm{min})\end{array}$} & \multirow{3}{*}{$\begin{array}{r}64.07 \\
2.56\end{array}$} \\
\hline G3-1 & 7.4 & 13.5 & 6.1 & & & \\
\hline G3-2 & 7.4 & 25.7 & 18.3 & & & \\
\hline G3-3 & 7.5 & 31.3 & 23.8 & & & \\
\hline
\end{tabular}

\begin{tabular}{|c|c|c|c|c|}
\hline & & & & \multirow[b]{3}{*}{$32.1 \%$} \\
\hline G3-4 & & & 0 & \\
\hline SS-3 & 7.3 & 11.6 & 4.3 & \\
\hline G4-1 & & & 0 & \multirow[b]{5}{*}{$0.0 \%$} \\
\hline G4-2 & & & 0 & \\
\hline G4-3 & & & 0 & \\
\hline G4-4 & & & 0 & \\
\hline SS-4 & & & 0 & \\
\hline$\overline{B 1}$ & & & 0 & $0.0 \%$ \\
\hline B2 & 106.5 & 109.0 & 2.5 & $1.5 \%$ \\
\hline B3 & & & 0 & $0.0 \%$ \\
\hline B4 & & & 0 & $0.0 \%$ \\
\hline B5 & 109.4 & 130.5 & 21.1 & $12.9 \%$ \\
\hline $\mathrm{B} 6$ & & & 0 & $0.0 \%$ \\
\hline DS in-1 & & & 0 & \multirow[b]{2}{*}{$0.0 \%$} \\
\hline DS in-2 & & & 0 & \\
\hline DS out-1 & 7.4 & 27.1 & 19.7 & \multirow[b]{4}{*}{$39.2 \%$} \\
\hline DS out-2 & 7.4 & 41.4 & 34 & \\
\hline DS out-3 & 7.8 & 17.1 & 9.3 & \\
\hline DS out-4 & 7.7 & 8.9 & 1.2 & \\
\hline Other & & & 0 & $0.0 \%$ \\
\hline
\end{tabular}

Total Collected

$141 \quad 86.1 \%$

\begin{tabular}{|c|c|c|c|c|}
\hline Initial Conditions & $\mathrm{RH}(\%)$ & Temperature $\left({ }^{\circ} \mathrm{C}\right)$ & & Atm. P. (mbar) \\
\hline 1-HUM/TMP-BDST & 88.4 & 22.4 & P-IN-BDST & 866 \\
\hline 2-HUM/TMP-BDST & 87.8 & 22.5 & & \\
\hline Final Conditions & $\mathrm{RH}(\%)$ & Temperature $\left({ }^{\circ} \mathrm{C}\right)$ & & Atm. P. (mbar) \\
\hline 1-HUM/TMP-BDST & 92.2 & 24 & P-IN-BDST & 868 \\
\hline 2-HUM/TMP-BDST & 90 & 23.9 & & \\
\hline
\end{tabular}


Single Patch q(film) Test Summary and Mass Balance - Smooth DS Surface

Test: Patch 5, $4 \mathrm{~cm}$ left of centerline, Crown

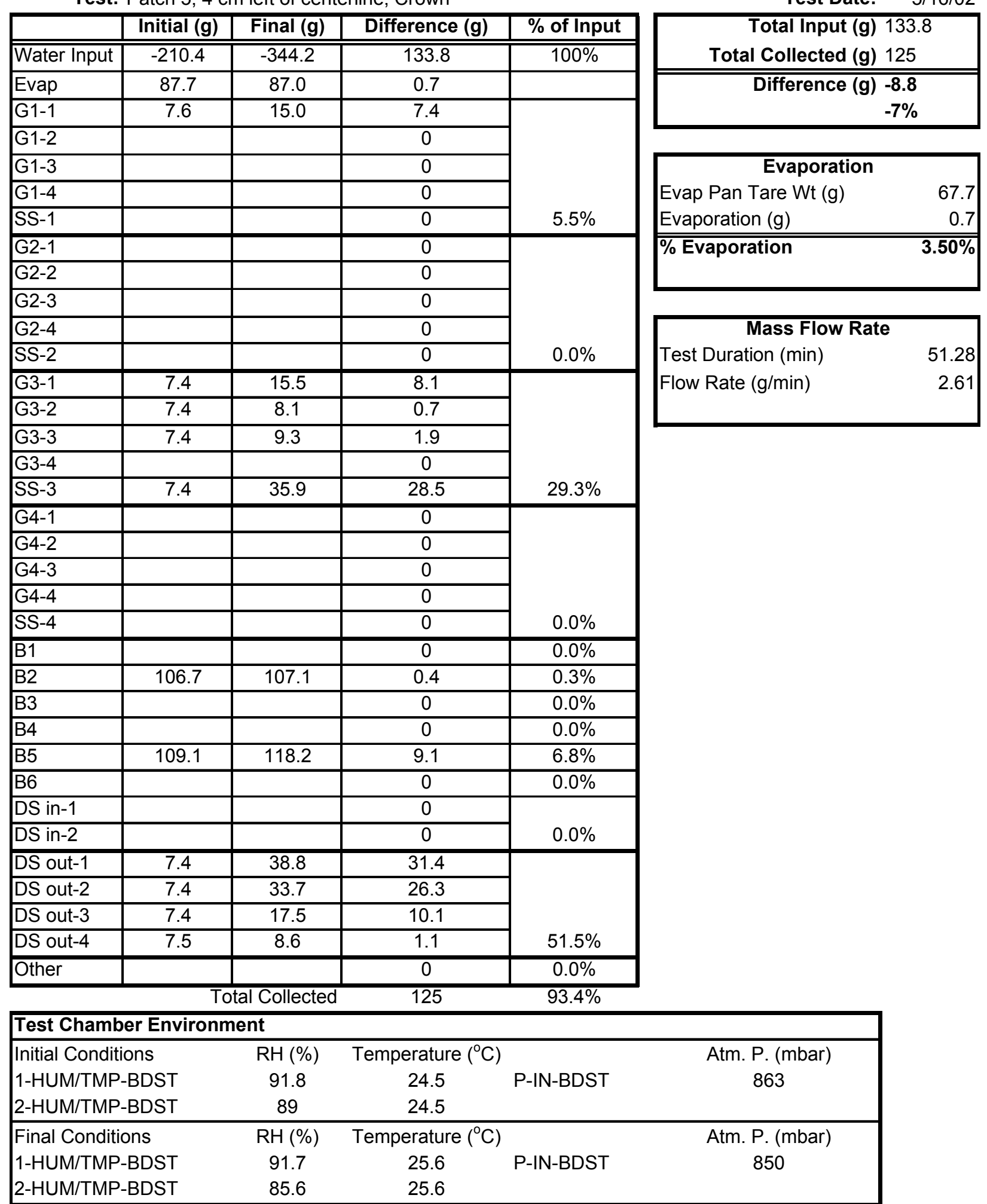


Single Patch q(film) Test Summary and Mass Balance - Smooth DS Surface

Test: Patch 5, $4 \mathrm{~cm}$ left of centerline, 16.5

\begin{tabular}{|c|c|c|c|c|}
\hline & Initial & Final & Difference & $\%$ of Input \\
\hline Water Input & $=-37.2$ & -210.3 & 17173.1 & $100 \%$ \\
\hline Evap & 94.3 & 93.6 & 0.7 & \\
\hline $\mathrm{G} 1-1$ & 7.6 & 7.8 & 0.2 & \multirow[b]{5}{*}{$2.3 \%$} \\
\hline$\overline{\mathrm{G} 1-2}$ & & & 0 & \\
\hline$\overline{\mathrm{G} 1-3}$ & & & 0 & \\
\hline$\overline{G 1-4}$ & & & 0 & \\
\hline SS-1 & 7.5 & 11.3 & 3.8 & \\
\hline$\overline{\mathrm{G} 2-1}$ & & & 0 & \multirow[b]{5}{*}{$0.0 \%$} \\
\hline$\overline{\mathrm{G} 2-2}$ & & & 0 & \\
\hline$\overline{\mathrm{G} 2-3}$ & & & 0 & \\
\hline $\bar{G} 2-4$ & & & 0 & \\
\hline SS-2 & & & 0 & \\
\hline$\overline{\mathrm{G} 3-1}$ & & & 0 & \multirow[b]{5}{*}{$0.1 \%$} \\
\hline$\overline{\mathrm{G}} 3-2$ & & & 0 & \\
\hline G3-3 & & & 0 & \\
\hline G3-4 & & & 0 & \\
\hline SS-3 & 7.6 & 7.7 & 0.1 & \\
\hline G4-1 & & & 0 & \multirow[b]{5}{*}{$0.0 \%$} \\
\hline G4-2 & & & 0 & \\
\hline G4-3 & & & 0 & \\
\hline$\overline{\mathrm{G}} 4-4$ & & & 0 & \\
\hline SS-4 & 7.7 & 7.7 & 0 & \\
\hline $\mathrm{B} 1$ & & & 0 & $0.0 \%$ \\
\hline $\bar{B} 2$ & 107.0 & 107.1 & 0.1 & $0.1 \%$ \\
\hline$\overline{B 3}$ & & & 0 & $0.0 \%$ \\
\hline B4 & 107.4 & 107.5 & 0.1 & $0.1 \%$ \\
\hline B5 & 117.4 & 301.9 & 184.5 & $106.6 \%$ \\
\hline$\overline{B 6}$ & & & 0 & $0.0 \%$ \\
\hline$\overline{D S}$ in-1 & & & 0 & \multirow[b]{2}{*}{$0.0 \%$} \\
\hline DS in-2 & & & 0 & \\
\hline DS out-1 & 7.6 & 20.0 & 12.4 & \multirow[b]{4}{*}{$10.9 \%$} \\
\hline DS out-2 & 7.6 & 13.0 & 5.4 & \\
\hline DS out-3 & 7.5 & 8.6 & 1.1 & \\
\hline DS out-4 & & & 0 & \\
\hline Other & & & $\overline{0}$ & $0.0 \%$ \\
\hline
\end{tabular}

Test Date: $\quad 5 / 31 / 02$

\begin{tabular}{|c|}
\hline $\begin{array}{r}\text { Total Input (g) } 173.1 \\
\text { Total Collected (g) } 207.7\end{array}$ \\
\hline $\begin{array}{r}\text { Difference }(\mathrm{g}) 34.6 \\
20 \%\end{array}$ \\
\hline
\end{tabular}

\begin{tabular}{|lr|}
\hline \multicolumn{2}{|c|}{ Evaporation } \\
Evap Pan Tare Wt, g & 66.7 \\
Evaporation, g & 0.7 \\
\hline \% Evaporation & $\mathbf{2 . 5 4 \%}$ \\
\hline
\end{tabular}

\begin{tabular}{|lr|}
\hline \multicolumn{2}{|c|}{ Mass Flow Rate } \\
Test Duration, min & 60.05 \\
Flow Rate, g/min & 2.88 \\
\hline
\end{tabular}

\begin{tabular}{|lccrc|}
\hline \multicolumn{7}{l}{ Test Chamber Environment } & & & \\
\hline Initial Conditions & $\mathrm{RH}(\%)$ & Temperature $\left({ }^{\circ} \mathrm{C}\right)$ & & Atm. P. (mbar) \\
1-HUM/TMP-BDST & 87.2 & 25.7 & P-IN-BDST & 870 \\
2-HUM/TMP-BDST & 87 & 25.3 & & \\
\hline Final Conditions & $\mathrm{RH}(\%)$ & Temperature $\left({ }^{\circ} \mathrm{C}\right)$ & & Atm. P. (mbar) \\
1-HUM/TMP-BDST & 90.9 & 25.9 & P-IN-BDST & 859 \\
2-HUM/TMP-BDST & 92.1 & 25.3 & & \\
\hline
\end{tabular}


Multiple Patch Test Summary and Mass Balance-Smooth DS Surface

Test: $81 \mathrm{~cm}$ left of DS center, Crown

\begin{tabular}{|c|c|c|c|c|c|c|}
\hline \multicolumn{5}{|c|}{ Test: $81 \mathrm{~cm}$ left of DS center, Crown } & Test Date & $6 / 13 / 02$ \\
\hline & Initial & Final & Difference & $\%$ of Input & \multicolumn{2}{|c|}{ Total Input 226.2} \\
\hline Water Input & $\overline{755.5}$ & $\overline{529.3}$ & 226.2 & $100 \%$ & \multicolumn{2}{|c|}{ Total Collected 164.2} \\
\hline Evap & 112.7 & 111.9 & 0.8 & & \multirow{2}{*}{\multicolumn{2}{|c|}{$\begin{aligned} \text { Difference } & -62.0 \\
& -27.4 \%\end{aligned}$}} \\
\hline $\mathrm{G} 1-1$ & 7.4 & 14.1 & 6.7 & \multirow[b]{5}{*}{$19.0 \%$} & & \\
\hline G1-2 & $\overline{7.4}$ & 43.7 & 36.3 & & & \\
\hline G1-3 & & & 0.0 & & \multicolumn{2}{|c|}{ Evaporation } \\
\hline G1-4 & & & 0.0 & & \multirow{2}{*}{$\begin{array}{l}\text { Evap Pan Tare Wt, g } \\
\text { Evaporation, g }\end{array}$} & 66.7 \\
\hline SS-1 & & & 0.0 & & & \\
\hline G2-1 & & & $\overline{0.0}$ & & \multirow[t]{2}{*}{ \% Evaporation } & $\overline{1.74 \%}$ \\
\hline G2-2 & & & 0.0 & & & \\
\hline G2-3 & & & 0.0 & & & \\
\hline G2-4 & & & 0.0 & & \multicolumn{2}{|c|}{ Mass Flow Rate } \\
\hline SS-2 & 7.4 & 7.5 & 0.1 & $0.0 \%$ & \multirow{3}{*}{$\begin{array}{l}\text { Test Duration, min } \\
\text { Flow Rate, } \mathrm{g} / \mathrm{min}\end{array}$} & 62.8 \\
\hline G3-1 & $\overline{7.3}$ & 17.1 & 9.8 & & & 3.60 \\
\hline G3-2 & 7.3 & 22.2 & 14.9 & & & \\
\hline G3-3 & & & 0.0 & & & \\
\hline
\end{tabular}

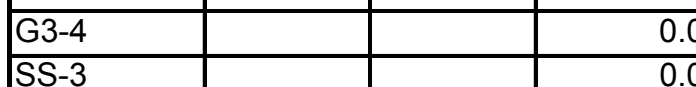

\begin{tabular}{|c|c|c|c|c|}
\hline SS-3 & & & 0.0 & $10.9 \%$ \\
\hline G4-1 & & & 0.0 & \multirow[b]{5}{*}{$0.0 \%$} \\
\hline G4-2 & & & 0.0 & \\
\hline G4-3 & & & 0.0 & \\
\hline G4-4 & & & 0.0 & \\
\hline SS-4 & & & 0.0 & \\
\hline B1 & 107.3 & 110.0 & 2.7 & $1.2 \%$ \\
\hline B2 & & & 0.0 & $0.0 \%$ \\
\hline B3 & & & 0.0 & $0.0 \%$ \\
\hline B4 & 107.1 & 110.6 & 3.5 & $1.5 \%$ \\
\hline B5 & & & 0.0 & $0.0 \%$ \\
\hline B6 & 109.7 & 109.9 & 0.2 & $0.1 \%$ \\
\hline DS in-1 & & & 0.0 & \multirow[b]{2}{*}{$0.0 \%$} \\
\hline DS in-2 & & & 0.0 & \\
\hline DS out-1 & 7.3 & 40.5 & 33.2 & \multirow[b]{4}{*}{$39.8 \%$} \\
\hline DS out-2 & 7.3 & 15.7 & 8.4 & \\
\hline DS out-3 & 7.3 & 26.9 & 19.6 & \\
\hline DS out-4 & 7.4 & 36.2 & 28.8 & \\
\hline Other & & & 0.0 & $0.0 \%$ \\
\hline
\end{tabular}

\begin{tabular}{|lcccc|}
\hline Test Chamber Environment & & & \\
\hline Initial Conditions & $\mathrm{RH}(\%)$ & Temperature $\left({ }^{\circ} \mathrm{C}\right)$ & & Atm. P. (mbar) \\
1-HUM/TMP-BDST & 82.3 & 22.7 & P-IN-BDST & 866 \\
2-HUM/TMP-BDST & 81.3 & 23 & & \\
\hline Final Conditions & $\mathrm{RH}(\%)$ & Temperature $\left({ }^{\circ} \mathrm{C}\right)$ & & Atm. P. (mbar) \\
1-HUM/TMP-BDST & 85.7 & 25.1 & P-IN-BDST & 856 \\
2-HUM/TMP-BDST & 86 & 25 & & \\
\hline
\end{tabular}


Multiple Patch Test Summary and Mass Balance-Smooth DS Surface

Test: $27 \mathrm{~cm}$ left of DS center, Crown

\begin{tabular}{|c|c|c|c|c|}
\hline & Initial & Final & Difference & $\%$ of Input \\
\hline Water Input & 710.1 & 433.2 & 276.9 & $100 \%$ \\
\hline Evap & 114.9 & 113.6 & 1.3 & \\
\hline$\overline{G 1-1}$ & 7.5 & 27.9 & 20.4 & \multirow[b]{5}{*}{$8.1 \%$} \\
\hline G1-2 & 7.5 & 9.6 & 2.1 & \\
\hline G1-3 & & & 0.0 & \\
\hline$\overline{G 1-4}$ & & & 0.0 & \\
\hline SS-1 & & & 0.0 & \\
\hline$\overline{G 2-1}$ & & & $\overline{0.0}$ & \multirow[b]{5}{*}{$0.0 \%$} \\
\hline G2-2 & & & 0.0 & \\
\hline G2-3 & & & 0.0 & \\
\hline G2-4 & & & 0.0 & \\
\hline SS-2 & & & 0.0 & \\
\hline G3-1 & 7.4 & 46.8 & $\overline{39.4}$ & \multirow[b]{5}{*}{$25.3 \%$} \\
\hline G3-2 & 7.4 & 23.5 & 16.1 & \\
\hline G3-3 & 7.4 & 21.9 & 14.5 & \\
\hline G3-4 & & & 0.0 & \\
\hline SS-3 & & & 0.0 & \\
\hline$\overline{G 4-1}$ & & & $\overline{0.0}$ & \multirow[b]{5}{*}{$0.0 \%$} \\
\hline G4-2 & & & 0.0 & \\
\hline G4-3 & & & 0.0 & \\
\hline G4-4 & & & 0.0 & \\
\hline SS-4 & & & 0.0 & \\
\hline $\mathrm{B} 1$ & & & 0.0 & $0.0 \%$ \\
\hline B2 & 106.5 & 108.3 & 1.8 & $0.7 \%$ \\
\hline B3 & & & 0.0 & $0.0 \%$ \\
\hline B4 & 107.7 & 110.4 & 2.7 & $1.0 \%$ \\
\hline B5 & 109.0 & 109.1 & 0.1 & $0.0 \%$ \\
\hline B6 & 109.5 & 117.0 & 7.5 & $2.7 \%$ \\
\hline DS in-1 & & & 0.0 & \multirow[b]{2}{*}{$0.0 \%$} \\
\hline DS in-2 & & & 0.0 & \\
\hline DS out-1 & 7.4 & 30.4 & 23.0 & \multirow{4}{*}{$34.7 \%$} \\
\hline DS out-2 & 7.4 & 43.8 & 36.4 & \\
\hline DS out- 3 & 7.5 & 39.8 & 32.3 & \\
\hline DS out-4 & 7.5 & 12.0 & 4.5 & \\
\hline Other & & & 0.0 & $0.0 \%$ \\
\hline
\end{tabular}

Test Date 6/13/02

\begin{tabular}{|l|}
\hline \multicolumn{2}{|c|}{ Total Input 276.9 } \\
Total Collected 200.8 \\
\hline \hline \multicolumn{2}{|c|}{ Difference $\mathbf{- 7 6 . 1}$} \\
\multicolumn{2}{|c|}{$\mathbf{2 7 . 5 \%}$} \\
\hline \multicolumn{2}{|c|}{ Evaporation } \\
Evap Pan Tare Wt, g \\
Evaporation, g \\
\hline \% Evaporation \\
\hline \multicolumn{2}{|c|}{1.3} \\
\hline \multicolumn{2}{|c|}{ Mass Flow Rate } \\
\hline Test Duration, min \\
Flow Rate, g/min \\
\hline
\end{tabular}

\begin{tabular}{|lcccc|}
\hline \multicolumn{7}{l}{ Test Chamber Environment } & & & \\
\hline Initial Conditions & $\mathrm{RH}(\%)$ & Temperature $\left({ }^{\circ} \mathrm{C}\right)$ & & Atm. P. (mbar) \\
1-HUM/TMP-BDST & 81.8 & 21.4 & P-IN-BDST & 870 \\
2-HUM/TMP-BDST & 84.4 & 21.2 & & \\
\hline Final Conditions & $\mathrm{RH}(\%)$ & Temperature $\left({ }^{\circ} \mathrm{C}\right)$ & & Atm. P. (mbar) \\
1-HUM/TMP-BDST & 87.8 & 23.2 & P-IN-BDST & 868 \\
2-HUM/TMP-BDST & 85.1 & 23.3 & & \\
\hline
\end{tabular}


Multiple Patch Test Summary and Mass Balance-Smooth DS Surface

Test: $27 \mathrm{~cm}$ right of DS center, Crown

Test Date 6/12/02

\begin{tabular}{|c|c|c|c|c|c|c|}
\hline & Initial & Final & Difference & $\%$ of Input & \multirow{2}{*}{\multicolumn{2}{|c|}{ Total Collected 152.3}} \\
\hline Water Input & 85.8 & -187.3 & 273.1 & \multirow[t]{2}{*}{$100 \%$} & & \\
\hline Evap & 87.1 & 85.4 & 1.7 & & \multirow{2}{*}{\multicolumn{2}{|c|}{$\begin{array}{r}\text { Difference }-120.8 \\
-44.2 \%\end{array}$}} \\
\hline$\overline{\mathrm{G} 1-1}$ & 7.4 & 22.7 & 15.3 & \multirow[b]{5}{*}{$9.6 \%$} & & \\
\hline$\overline{\mathrm{G} 1-2}$ & 7.4 & 17.9 & 10.5 & & \\
\hline G1-3 & 7.5 & 7.9 & 0.4 & & \multicolumn{2}{|c|}{ Evaporation } \\
\hline $\mathrm{G} 1-4$ & & & 0 & & \multirow{2}{*}{$\begin{array}{l}\text { Evap Pan Tare Wt, g } \\
\text { Evaporation, g }\end{array}$} & \multirow{2}{*}{$\begin{array}{r}66.7 \\
1.7 \\
\end{array}$} \\
\hline SS-1 & & & 0 & & & \\
\hline G2-1 & & & 0 & \multirow[b]{5}{*}{$0.0 \%$} & \multirow[t]{2}{*}{ \% Evaporation } & \multirow[t]{2}{*}{$8.33 \%$} \\
\hline G2-2 & & & 0 & & & \\
\hline G2-3 & & & 0 & & & \\
\hline G2-4 & & & 0 & & \multicolumn{2}{|c|}{ Mass Flow Rate } \\
\hline SS-2 & & & 0 & & \multirow{3}{*}{$\begin{array}{l}\text { Test Duration, min } \\
\text { Flow Rate, g/min }\end{array}$} & \multirow{3}{*}{$\begin{array}{r}65.68 \\
4.16\end{array}$} \\
\hline G3-1 & 7.4 & 17.1 & 9.7 & & & \\
\hline G3-2 & 7.3 & 41 & 33.7 & & & \\
\hline G3-3 & & & 0 & & & \\
\hline
\end{tabular}

G3-4 (SS)

SS-3

G4-1

G4-2

G4-3

G4-4

SS-4

$\mathrm{B} 1$

$\mathrm{B} 2$

B3

$\mathrm{B}$

B6

\begin{tabular}{|c|c|c|c|c|}
\hline B6 & 109.6 & 114.4 & 4.8 & $1.8 \%$ \\
\hline DS in-1 & & & 0 & \multirow[b]{2}{*}{$0.0 \%$} \\
\hline$\overline{D S}$ in-2 & & & 0 & \\
\hline DS out-1 & 7.4 & 34.5 & 27.1 & \multirow[b]{4}{*}{$22.6 \%$} \\
\hline DS out-2 & 7.5 & 15.1 & 7.6 & \\
\hline DS out- 3 & 7.5 & 7.9 & 0.4 & \\
\hline DS out-4 & 7.6 & 34.2 & 26.6 & \\
\hline Other & & & 0 & $0.0 \%$ \\
\hline
\end{tabular}

\begin{tabular}{|lcccc|}
\hline \multicolumn{7}{|l|}{ Test Chamber Environment } & & & \\
\hline Initial Conditions & $\mathrm{RH}(\%)$ & Temperature $\left({ }^{\circ} \mathrm{C}\right)$ & & Atm. P. (mbar) \\
1-HUM/TMP-BDST & 81.6 & 25.4 & P-IN-BDST & 861 \\
2-HUM/TMP-BDST & 81.3 & 25.4 & & \\
\hline Final Conditions & $\mathrm{RH}(\%)$ & Temperature $\left({ }^{\circ} \mathrm{C}\right)$ & & Atm. P. (mbar) \\
1-HUM/TMP-BDST & 87.5 & 25.3 & P-IN-BDST & 865 \\
2-HUM/TMP-BDST & 86.6 & 25.4 & & \\
\hline
\end{tabular}


Multiple Patch Test Summary and Mass Balance-Smooth DS Surface

Test: $81 \mathrm{~cm}$ right of DS center, Crown

Test Date 6/12/02

\begin{tabular}{|c|c|c|c|c|c|c|}
\hline & Initial & Final & Difference & $\%$ of Input & \multirow{2}{*}{\multicolumn{2}{|c|}{ Total Collected 192.1}} \\
\hline Water Input & -0.5 & -276.6 & 276.1 & $100 \%$ & & \\
\hline Evap & 89.4 & 88.1 & 1.3 & & \multirow{2}{*}{\multicolumn{2}{|c|}{$\begin{aligned} & \text { Difference }-84 \\
&-30.4 \%\end{aligned}$}} \\
\hline G1-1 & 7.3 & 12.5 & 5.2 & \multirow[b]{5}{*}{$17.3 \%$} & & \\
\hline G1-2 & 7.3 & 33.5 & 26.2 & & & \\
\hline G1-3 & 7.4 & 23.8 & 16.4 & & \multicolumn{2}{|c|}{ Evaporation } \\
\hline G1-4 & & & 0.0 & & \multirow{4}{*}{\multicolumn{2}{|c|}{$\begin{array}{lr}\text { Evap Pan Tare Wt, g } & 66.7 \\
\text { Evaporation, g } & 1.3 \\
\text { \% Evaporation } & \mathbf{5 . 7 3 \%}\end{array}$}} \\
\hline SS-1 & & & 0.0 & & & \\
\hline G2-1 & & & $\overline{0.0}$ & \multirow[b]{5}{*}{$0.0 \%$} & & \\
\hline G2-2 & & & 0.0 & & & \\
\hline G2-3 & & & 0.0 & & & \\
\hline G2-4 & & & 0.0 & & \multicolumn{2}{|c|}{ Mass Flow Rate } \\
\hline SS-2 & & & 0.0 & & \multirow{3}{*}{$\begin{array}{l}\text { Test Duration, min } \\
\text { Flow Rate, g/min }\end{array}$} & 72.38 \\
\hline G3-1 & 7.5 & 25.3 & 17.8 & & & 3.81 \\
\hline G3-2 & $\overline{7.3}$ & 27.5 & 20.2 & & & \\
\hline G3-3 (SS) & 7.5 & 8.5 & 1.0 & & & \\
\hline
\end{tabular}

\begin{tabular}{|c|c|c|c|c|}
\hline & & & & \multirow[b]{3}{*}{$18.4 \%$} \\
\hline G3-4 & 7.5 & 8.2 & 0.7 & \\
\hline SS-3 & 7.7 & 18.9 & 11.2 & \\
\hline G4-1 & & & 0.0 & \multirow[b]{5}{*}{$0.0 \%$} \\
\hline G4-2 & & & 0.0 & \\
\hline G4-3 & & & 0.0 & \\
\hline G4-4 & & & 0.0 & \\
\hline SS-4 & & & 0.0 & \\
\hline $\mathrm{B} 1$ & & & $\overline{0.0}$ & $0.0 \%$ \\
\hline B2 & 106.5 & 106.8 & 0.3 & $0.1 \%$ \\
\hline B3 & & & 0.0 & $0.0 \%$ \\
\hline B4 & 107.0 & 107.1 & 0.1 & $0.0 \%$ \\
\hline B5 & 109.1 & 113.5 & 4.4 & $1.6 \%$ \\
\hline B6 & 109.6 & 115.6 & 6.0 & $2.2 \%$ \\
\hline DS in-1 & & & 0.0 & \multirow[b]{2}{*}{$0.0 \%$} \\
\hline DS in-2 & & & 0.0 & \\
\hline DS out-1 & 8.5 & 9.9 & 1.4 & \multirow[b]{4}{*}{$29.9 \%$} \\
\hline DS out-2 & 7.4 & 23.4 & 16.0 & \\
\hline DS out-3 & 7.3 & 38.2 & 30.9 & \\
\hline DS out-4 & 7.6 & 41.9 & 34.3 & \\
\hline Other & & & 0.0 & $0.0 \%$ \\
\hline
\end{tabular}

\begin{tabular}{|lcccc|}
\hline \multicolumn{7}{|l|}{ Test Chamber Environment } & & & \\
\hline Initial Conditions & $\mathrm{RH}(\%)$ & Temperature $\left({ }^{\circ} \mathrm{C}\right)$ & & Atm. P. (mbar) \\
1-HUM/TMP-BDST & 82.6 & 22.4 & P-IN-BDST & 858 \\
2-HUM/TMP-BDST & 81.9 & 22.3 & & \\
\hline Final Conditions & $\mathrm{RH}(\%)$ & Temperature $\left({ }^{\circ} \mathrm{C}\right)$ & & Atm. P. (mbar) \\
1-HUM/TMP-BDST & 82.1 & 24.7 & P-IN-BDST & 857 \\
2-HUM/TMP-BDST & 80.5 & 24.8 & & \\
\hline
\end{tabular}


Multiple Patch Test Summary and Mass Balance-Smooth DS Surface

Test: $81 \mathrm{~cm}$ left of DS center, 16.5

\begin{tabular}{|c|c|c|c|c|}
\hline & Initial & Final & Difference & $\%$ of Input \\
\hline Water Input & 838.9 & 589.8 & 249.1 & $100 \%$ \\
\hline Evap & 109.3 & 107.2 & 2.1 & \\
\hline$\overline{\mathrm{G} 1-1}$ & 7.6 & 52.3 & 44.7 & \multirow[b]{5}{*}{$74.4 \%$} \\
\hline$\overline{G 1-2}$ & 7.5 & 44.5 & 37.0 & \\
\hline$\overline{\mathrm{G} 1-3}$ & 7.6 & 62.1 & 54.5 & \\
\hline G1-4 & 7.5 & 55.6 & 48.1 & \\
\hline$\overline{\mathrm{G} 1-5}$ & 7.6 & 8.6 & 1.0 & \\
\hline$\overline{\mathrm{G} 2-1}$ & & & $\overline{0.0}$ & \multirow[b]{5}{*}{$0.0 \%$} \\
\hline G2-2 & & & 0.0 & \\
\hline G2-3 & & & 0.0 & \\
\hline G2-4 & & & 0.0 & \\
\hline SS-2 & 7.6 & 7.6 & 0.0 & \\
\hline G3-1 & & & 0.0 & \multirow[b]{5}{*}{$0.0 \%$} \\
\hline G3-2 & & & 0.0 & \\
\hline G3-3 & & & 0.0 & \\
\hline G3-4 & & & 0.0 & \\
\hline SS-3 & 7.5 & 7.5 & 0.0 & \\
\hline G4-1 & & & 0.0 & \multirow[b]{5}{*}{$0.0 \%$} \\
\hline G4-2 & & & 0.0 & \\
\hline G4-3 & & & 0.0 & \\
\hline G4-4 & & & 0.0 & \\
\hline SS-4 & & & 0.0 & \\
\hline B1 & 107.6 & 107.7 & 0.1 & $0.0 \%$ \\
\hline B2 & & & 0.0 & $0.0 \%$ \\
\hline B3 & & & 0.0 & $0.0 \%$ \\
\hline B4 & 107.3 & 107.3 & 0.0 & $0.0 \%$ \\
\hline B5 & & & 0.0 & $0.0 \%$ \\
\hline $\mathrm{B} 6$ & 109.9 & 109.9 & 0.0 & $0.0 \%$ \\
\hline DS in-1 & & & 0.0 & \multirow[b]{2}{*}{$0.0 \%$} \\
\hline DS in-2 & & & 0.0 & \\
\hline DS out-1 & 7.5 & 7.6 & 0.1 & \multirow[b]{4}{*}{$2.2 \%$} \\
\hline DS out-2 & 7.5 & 9.6 & 2.1 & \\
\hline DS out-3 & 7.6 & 10.8 & 3.2 & \\
\hline DS out-4 & & & 0.0 & \\
\hline Other G1-5 & & & 0.0 & $0.0 \%$ \\
\hline
\end{tabular}

Test Date 6/13/02

Total Input 249.1

Total Collected 190.8

Difference -58.3 $-23 \%$

\section{Evaporation}

Evap Pan Tare Wt, g $\quad 66.7$

Evaporation, g $\quad 2.1$

\% Evaporation $\quad 4.93 \%$

Mass Flow Rate

Test Duration, $\min \quad 66.02$

Flow Rate, $\mathrm{g} / \mathrm{min} \quad 3.77$

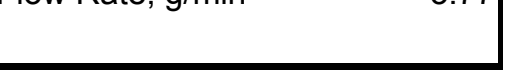

Test Chamber Environment

\begin{tabular}{|lcccc|}
\hline Initial Conditions & $\mathrm{RH}(\%)$ & Temperature $\left({ }^{\circ} \mathrm{C}\right)$ & Atm. P. (mbar) \\
1-HUM/TMP-BDST & 85 & 25.4 & P-IN-BDST & 856 \\
2-HUM/TMP-BDST & 81.7 & 25.8 & & \\
\hline Final Conditions & $\mathrm{RH}(\%)$ & Temperature $\left({ }^{\circ} \mathrm{C}\right)$ & & Atm. P. (mbar) \\
1-HUM/TMP-BDST & 80.2 & 26.2 & P-IN-BDST & 863 \\
2-HUM/TMP-BDST & 80.1 & 26.3 & & \\
\hline
\end{tabular}


Multiple Patch Test Summary and Mass Balance-Smooth DS Surface

Test: $27 \mathrm{~cm}$ left of DS center, 16.5

\begin{tabular}{|c|c|c|c|c|}
\hline & Initial & Final & Difference & $\%$ of Input \\
\hline Water Input & 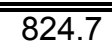 & $\overline{589.0}$ & 235.7 & $100 \%$ \\
\hline Evap & 106.6 & 106.2 & 0.4 & \\
\hline$\overline{\mathrm{G} 1-1}$ & 7.6 & 30.9 & 23.3 & \multirow[b]{5}{*}{$63.9 \%$} \\
\hline$\overline{\mathrm{G} 1-2}$ & 7.5 & 42.9 & 35.4 & \\
\hline$\overline{\mathrm{G} 1-3}$ & 7.5 & 53.9 & 46.4 & \\
\hline G1-4 & 7.4 & 52.9 & 45.5 & \\
\hline SS-1 & & & 0.0 & \\
\hline $\mathrm{G} 2-1$ & & & $\overline{0.0}$ & \multirow[b]{5}{*}{$0.0 \%$} \\
\hline G2-2 & & & 0.0 & \\
\hline G2-3 & & & 0.0 & \\
\hline G2-4 & & & 0.0 & \\
\hline SS-2 & & & 0.0 & \\
\hline$\overline{\text { G3-1 }}$ & & & $\overline{0.0}$ & \multirow[b]{5}{*}{$0.0 \%$} \\
\hline G3-2 & & & 0.0 & \\
\hline G3-3 & & & 0.0 & \\
\hline G3-4 & & & 0.0 & \\
\hline SS-3 & 7.5 & 7.6 & 0.1 & \\
\hline$\overline{\mathrm{G} 4-1}$ & & & $\overline{0.0}$ & \multirow[b]{5}{*}{$0.0 \%$} \\
\hline G4-2 & & & 0.0 & \\
\hline G4-3 & & & 0.0 & \\
\hline G4-4 & & & 0.0 & \\
\hline SS-4 & & & 0.0 & \\
\hline $\mathrm{B} 1$ & & & 0.0 & $0.0 \%$ \\
\hline B2 & 106.8 & 108.2 & 1.4 & $0.6 \%$ \\
\hline B3 & & & 0.0 & $0.0 \%$ \\
\hline B4 & 107.4 & 107.8 & 0.4 & $0.2 \%$ \\
\hline B5 & & & 0.0 & $0.0 \%$ \\
\hline $\mathrm{B} 6$ & 110.0 & 110.5 & 0.5 & $0.2 \%$ \\
\hline DS in-1 & & & 0.0 & \multirow[b]{2}{*}{$0.0 \%$} \\
\hline DS in-2 & & & 0.0 & \\
\hline DS out-1 & 7.5 & 11.7 & 4.2 & \multirow[b]{4}{*}{$14.8 \%$} \\
\hline DS out- 2 & 7.5 & 23.2 & 15.7 & \\
\hline DS out-3 & 7.5 & 22.4 & 14.9 & \\
\hline DS out-4 & & & 0.0 & \\
\hline Other & & & 0.0 & $0.0 \%$ \\
\hline
\end{tabular}

Test Date 6/13/02

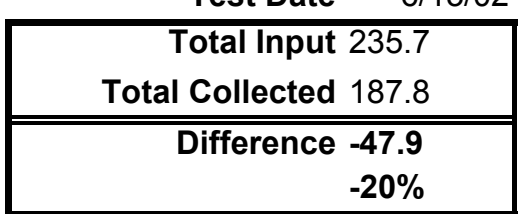

\begin{tabular}{|lr|}
\hline \multicolumn{2}{|c|}{ Evaporation } \\
Evap Pan Tare Wt, g & 66.7 \\
Evaporation, g & 0.4 \\
\hline \% Evaporation & $\mathbf{1 . 0 0 \%}$ \\
\hline
\end{tabular}

\begin{tabular}{|lr|}
\hline \multicolumn{2}{|c|}{ Mass Flow Rate } \\
Test Duration, $\min$ & 63.88 \\
Flow Rate, g/min & 3.69 \\
& \\
\hline
\end{tabular}

Test Chamber Environment

\begin{tabular}{|lcccc|}
\hline Initial Conditions & $\mathrm{RH}(\%)$ & Temperature $\left({ }^{\circ} \mathrm{C}\right)$ & & Atm. P. (mbar) \\
1-HUM/TMP-BDST & 83.7 & 26.8 & P-IN-BDST & 862 \\
2-HUM/TMP-BDST & 85.4 & 26.8 & & \\
\hline Final Conditions & $\mathrm{RH}(\%)$ & Temperature $\left({ }^{\circ} \mathrm{C}\right)$ & & Atm. P. (mbar) \\
1-HUM/TMP-BDST & 91.7 & 28 & P-IN-BDST & 871 \\
2-HUM/TMP-BDST & 92 & 27.8 & & \\
\hline
\end{tabular}


Multiple Patch Test Summary and Mass Balance-Smooth DS Surface

Test: $27 \mathrm{~cm}$ right of DS center, 16.5

\begin{tabular}{|c|c|c|c|c|}
\hline & Initial & Final & Difference & $\%$ of Input \\
\hline Water Input & 790.3 & 543.2 & 247.1 & $100 \%$ \\
\hline Evap & 89.6 & 89.2 & 0.4 & \\
\hline $\mathrm{G} 1-1$ & 65.1 & 233.2 & 168.1 & \multirow[b]{5}{*}{$68.0 \%$} \\
\hline G1-2 & & & 0.0 & \\
\hline G1-3 & & & 0.0 & \\
\hline G1-4 & & & 0.0 & \\
\hline SS-1 & & & 0.0 & \\
\hline G2-1 & & & 0.0 & \multirow[b]{5}{*}{$0.0 \%$} \\
\hline G2-2 & & & 0.0 & \\
\hline G2-3 & & & 0.0 & \\
\hline G2-4 & & & 0.0 & \\
\hline SS-2 & & & 0.0 & \\
\hline G3-1 & 41.6 & 41.9 & 0.3 & \multirow[b]{5}{*}{$0.1 \%$} \\
\hline G3-2 & & & 0.0 & \\
\hline G3-3 & & & 0.0 & \\
\hline G3-4 & & & 0.0 & \\
\hline SS-3 & & & 0.0 & \\
\hline G4-1 & & & 0.0 & \multirow[b]{5}{*}{$0.0 \%$} \\
\hline G4-2 & & & 0.0 & \\
\hline G4-3 & & & 0.0 & \\
\hline G4-4 & & & 0.0 & \\
\hline SS-4 & & & 0.0 & \\
\hline B1 & & & 0.0 & $0.0 \%$ \\
\hline $\mathrm{B} 2$ & 107.0 & 108.5 & 1.5 & $0.6 \%$ \\
\hline B3 & & & 0.0 & $0.0 \%$ \\
\hline B4 & 107.5 & 107.7 & 0.2 & $0.1 \%$ \\
\hline B5 & 109.4 & 110.8 & 1.4 & $0.6 \%$ \\
\hline B6 & 110.2 & 110.5 & 0.3 & $0.1 \%$ \\
\hline DS in-1 & & & 0.0 & \multirow[b]{2}{*}{$0.0 \%$} \\
\hline DS in-2 & & & 0.0 & \\
\hline DS out-1 & 57.1 & 100.6 & 43.5 & \multirow[b]{4}{*}{$17.7 \%$} \\
\hline DS out-2 & 7.8 & 8.1 & 0.3 & \\
\hline DS out-3 & & & 0.0 & \\
\hline DS out-4 & & & 0.0 & \\
\hline Other & & & 0.0 & $0.0 \%$ \\
\hline
\end{tabular}

Test Date 6/14/02

Total Input 247.1

Total Collected 215.6

Difference -31.5

$-13 \%$

Evaporation

Evap Pan Tare Wt, g $\quad 66.7$

Evaporation, $g$

0.4

\% Evaporation $1.75 \%$

\begin{tabular}{|lr|}
\hline \multicolumn{2}{|c|}{ Mass Flow Rate } \\
Test Duration, min & 67.63 \\
Flow Rate, g/min & 3.65 \\
\hline
\end{tabular}

Test Chamber Environment

\begin{tabular}{lcccc}
\hline Initial Conditions & $\mathrm{RH}(\%)$ & Temperature $\left({ }^{\circ} \mathrm{C}\right)$ & Atm. P. (mbar) \\
1-HUM/TMP-BDST & 85.2 & 23 & P-IN-BDST & 882 \\
2-HUM/TMP-BDST & 87.8 & 23.1 & & Atm. P. (mbar) \\
\hline Final Conditions & $\mathrm{RH}(\%)$ & Temperature $\left({ }^{\circ} \mathrm{C}\right)$ & & 873 \\
1-HUM/TMP-BDST & 95.7 & 25 & P-IN-BDST & \\
2-HUM/TMP-BDST & 93.9 & 25 & & \\
\hline
\end{tabular}


Multiple Patch Test Summary and Mass Balance-Smooth DS Surface

Test: $81 \mathrm{~cm}$ right of DS center, 16.5

\begin{tabular}{|c|c|c|c|c|}
\hline & Initial & Final & Difference & $\%$ of Input \\
\hline Water Input & 811.4 & $\overline{5990.6}$ & 2220.8 & $100 \%$ \\
\hline Evap & 152.3 & 151.4 & 0.9 & \\
\hline $\mathrm{G} 1-1$ & 7.4 & 57 & 49.6 & \multirow[b]{5}{*}{$79.2 \%$} \\
\hline G1-2 & 7.4 & 61.7 & 54.3 & \\
\hline G1-3 & 7.8 & 43.8 & 36 & \\
\hline $\mathrm{G} 1-4$ & 7.3 & 38.7 & $\overline{31.4}$ & \\
\hline SS-1 (G1-5) & 7.5 & 11 & 3.5 & \\
\hline G2-1 & & & 0 & \multirow[b]{5}{*}{$0.0 \%$} \\
\hline G2-2 & & & 0 & \\
\hline G2-3 & & & 0 & \\
\hline G2-4 & & & 0 & \\
\hline SS-2 & & & 0 & \\
\hline G3-1 & & & 0 & \multirow[b]{5}{*}{$0.0 \%$} \\
\hline G3-2 & & & 0 & \\
\hline G3-3 & & & 0 & \\
\hline G3-4 & & & 0 & \\
\hline SS-3 & & & 0 & \\
\hline G4-1 & 7.6 & 8.2 & 0.6 & \multirow[b]{5}{*}{$0.3 \%$} \\
\hline G4-2 & & & 0 & \\
\hline G4-3 & & & 0 & \\
\hline G4-4 & & & 0 & \\
\hline SS-4 & & & 0 & \\
\hline $\mathrm{B} 1$ & & & 0 & $0.0 \%$ \\
\hline B2 & & & 0 & $0.0 \%$ \\
\hline B3 & 105.8 & 107.9 & 2.1 & $1.0 \%$ \\
\hline B4 & & & 0 & $0.0 \%$ \\
\hline B5 & 108.5 & 110.4 & 1.9 & $0.9 \%$ \\
\hline B6 & & & 0 & $0.0 \%$ \\
\hline DS in-1 & & & 0 & \multirow[b]{2}{*}{$0.0 \%$} \\
\hline DS in-2 & & & 0 & \\
\hline DS out-1 & 7.3 & 22.7 & 15.4 & \multirow[b]{4}{*}{$11.1 \%$} \\
\hline DS out- 2 & 7.6 & 15.1 & 7.5 & \\
\hline DS out-3 & 7.6 & 9.1 & 1.5 & \\
\hline DS out-4 & & & 0 & \\
\hline Other & & & 0 & $0.0 \%$ \\
\hline
\end{tabular}

Test Date 6/3/02

\begin{tabular}{|r|}
\hline Total Input 220.8 \\
Total Collected 203.8 \\
\hline \hline Difference -17 \\
$-8 \%$ \\
\hline
\end{tabular}

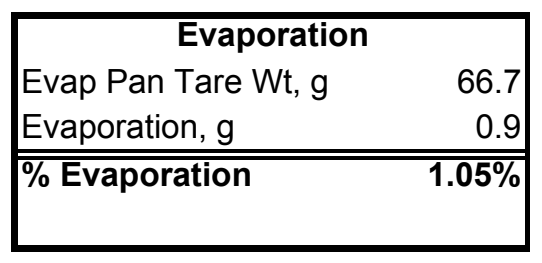

\begin{tabular}{|lr|}
\hline \multicolumn{2}{|c|}{ Mass Flow Rate } \\
Test Duration, min & 60 \\
Flow Rate, $\mathrm{g} / \mathrm{min}$ & 3.68 \\
\hline
\end{tabular}

\begin{tabular}{|lcccc|}
\hline \multicolumn{7}{|l|}{ Test Chamber Environment } & & & \\
\hline Initial Conditions & $\mathrm{RH}(\%)$ & Temperature $\left({ }^{\circ} \mathrm{C}\right)$ & & Atm. P. (mbar) \\
1-HUM/TMP-BDST & 89.9 & 25.9 & P-IN-BDST & 865 \\
2-HUM/TMP-BDST & 90.8 & 25.5 & & \\
\hline Final Conditions & $\mathrm{RH}(\%)$ & Temperature $\left({ }^{\circ} \mathrm{C}\right)$ & & Atm. P. (mbar) \\
1-HUM/TMP-BDST & 91.5 & 25.8 & P-IN-BDST & 875 \\
2-HUM/TMP-BDST & 92.3 & 25.5 & & \\
\hline
\end{tabular}

SN-M\&O-SCI-043-V1, pages 150-151 
Multiple Patch Test Summary and Mass Balance-Smooth DS Surface

Test: $81 \mathrm{~cm}$ right of DS center, 33

Test Date 6/3/02

\begin{tabular}{|c|c|c|c|c|}
\hline & Initial & Final & Difference & $\%$ of Input \\
\hline Water Input & 693.1 & $\overline{5669.4}$ & 123.7 & 1.000 \\
\hline Evap & 153.2 & 152.7 & 0.5 & \\
\hline $\mathrm{G} 1-1$ & 7.4 & 57.1 & 49.7 & \multirow[b]{5}{*}{0.871} \\
\hline$\overline{\mathrm{G} 1-2}$ & 7.4 & 52.2 & 44.8 & \\
\hline G1-3 & 7.4 & 9.2 & 1.8 & \\
\hline G1-4 & $\overline{7.4}$ & 18.9 & 11.5 & \\
\hline SS-1 & & & 0.0 & \\
\hline G2-1 & & & 0.0 & \multirow[b]{5}{*}{0.000} \\
\hline G2-2 & & & 0.0 & \\
\hline G2-3 & & & 0.0 & \\
\hline G2-4 & & & 0.0 & \\
\hline SS-2 & & & 0.0 & \\
\hline$\overline{\text { G3-1 }}$ & & & 0.0 & \multirow[b]{5}{*}{0.000} \\
\hline$\overline{\text { G3-2 }}$ & & & $\overline{0.0}$ & \\
\hline G3-3 & & & 0.0 & \\
\hline G3-4 & & & 0.0 & \\
\hline SS-3 & & & 0.0 & \\
\hline$\overline{\mathrm{G} 4-1}$ & $\overline{7.6}$ & $\overline{7.6}$ & 0.0 & \multirow[b]{5}{*}{0.000} \\
\hline G4-2 & & & 0.0 & \\
\hline G4-3 & & & 0.0 & \\
\hline$\overline{\mathrm{G} 4-4}$ & & & 0.0 & \\
\hline SS-4 & & & 0.0 & \\
\hline$\overline{\mathrm{B} 1}$ & & & 0.0 & 0.000 \\
\hline B2 & & & 0.0 & 0.000 \\
\hline B3 & 105.7 & 106.3 & 0.6 & 0.005 \\
\hline B4 & & & 0.0 & 0.000 \\
\hline B5 & 108.9 & 109.4 & 0.5 & 0.004 \\
\hline B6 & & & 0.0 & 0.000 \\
\hline$\overline{D S}$ in-1 & & & 0.0 & \multirow[b]{2}{*}{0.000} \\
\hline DS in-2 & & & 0.0 & \\
\hline DS out-1 & $\overline{7.4}$ & 9.4 & 2.0 & \multirow[b]{4}{*}{0.020} \\
\hline DS out-2 & 7.4 & 7.9 & 0.5 & \\
\hline DS out-3 & & & 0.0 & \\
\hline DS out-4 & & & 0.0 & \\
\hline Other & & & 0.0 & 0.000 \\
\hline
\end{tabular}

Total Input 123.7

Total Collected 111.4

Difference -12.3 $-10 \%$

\begin{tabular}{|lr|}
\hline Evap Pan Tare Wt, g & 66.7 \\
Evaporation, g & 0.5 \\
\hline \% Evaporation & $\mathbf{0 . 5 8 \%}$ \\
\hline
\end{tabular}

\begin{tabular}{|lr|}
\hline \multicolumn{2}{|c|}{ Mass Flow Rate } \\
Test Duration, min & 30 \\
Flow Rate, g/min & 4.12 \\
\hline
\end{tabular}

Test Chamber Environment

\begin{tabular}{|lcccc|}
\hline Initial Conditions & $\mathrm{RH}(\%)$ & Temperature $\left({ }^{\circ} \mathrm{C}\right)$ & & Atm. P. (mbar) \\
1-HUM/TMP-BDST & 86.3 & 25.6 & P-IN-BDST & 861 \\
2-HUM/TMP-BDST & 86.5 & 25.6 & & \\
\hline Final Conditions & $\mathrm{RH}(\%)$ & Temperature $\left({ }^{\circ} \mathrm{C}\right)$ & & Atm. P. (mbar) \\
1-HUM/TMP-BDST & 90.7 & 25.8 & P-IN-BDST & 864 \\
2-HUM/TMP-BDST & 90.6 & 25.8 & & \\
\hline
\end{tabular}

SN-M\&O-SCl-043-V1, pages 148-149 
Multiple Patch Test Summary and Mass Balance-Smooth DS Surface

Test: $27 \mathrm{~cm}$ right of DS center, 33

\begin{tabular}{|c|c|c|c|c|}
\hline & Initial & Final & Difference & $\%$ of Input \\
\hline Water Input & 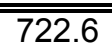 & 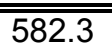 & 140.3 & $100 \%$ \\
\hline Evap & 154.4 & 153.6 & 0.8 & \\
\hline$\overline{\mathrm{G} 1-1}$ & 7.4 & 58.4 & 51.0 & \multirow[b]{5}{*}{$86.3 \%$} \\
\hline$\overline{\mathrm{G} 1-2}$ & 7.2 & 61.0 & 53.8 & \\
\hline$\overline{\mathrm{G} 1-3}$ & 7.2 & 20.6 & 13.4 & \\
\hline$\overline{\mathrm{G} 1-4}$ & 7.2 & 10.1 & 2.9 & \\
\hline SS-1 & & & 0.0 & \\
\hline$\overline{\mathrm{G} 2-1}$ & & & $\overline{0.0}$ & \multirow[b]{5}{*}{$0.0 \%$} \\
\hline G2-2 & & & 0.0 & \\
\hline $\mathrm{G} 2-3$ & & & 0.0 & \\
\hline $\bar{G} 2-4$ & & & 0.0 & \\
\hline SS-2 & & & 0.0 & \\
\hline G3-1 & & & $\overline{0.0}$ & \multirow[b]{5}{*}{$0.0 \%$} \\
\hline G3-2 & & & 0.0 & \\
\hline G3-3 & & & 0.0 & \\
\hline G3-4 & & & 0.0 & \\
\hline SS-3 & & & 0.0 & \\
\hline G4-1 & & & $\overline{0.0}$ & \multirow[b]{5}{*}{$0.0 \%$} \\
\hline G4-2 & & & 0.0 & \\
\hline G4-3 & & & 0.0 & \\
\hline G4-4 & & & 0.0 & \\
\hline SS-4 & & & 0.0 & \\
\hline $\mathrm{B} 1$ & & & 0.0 & $0.0 \%$ \\
\hline B2 & 106.7 & 106.8 & 0.1 & $0.1 \%$ \\
\hline B3 & & & 0.0 & $0.0 \%$ \\
\hline B4 & & & 0.0 & $0.0 \%$ \\
\hline B5 & 109.2 & 109.3 & 0.1 & $0.1 \%$ \\
\hline B6 & & & 0.0 & $0.0 \%$ \\
\hline DS in-1 & & & 0.0 & \multirow[b]{2}{*}{$0.0 \%$} \\
\hline DS in-2 & & & 0.0 & \\
\hline DS out-1 & 7.5 & 9.2 & 1.7 & \multirow[b]{4}{*}{$1.7 \%$} \\
\hline DS out-2 & 7.3 & 8.0 & 0.7 & \\
\hline DS out-3 & & & 0.0 & \\
\hline DS out-4 & & & 0.0 & \\
\hline Other & & & $\overline{0.0}$ & $0.0 \%$ \\
\hline
\end{tabular}

Test Date 6/3/02

Total Input 140.3

Total Collected 123.7

Difference -16.6

$-12 \%$

Evaporation

Evap Pan Tare Wt, g $\quad 66.7$

Evaporation, g

0.8

\% Evaporation $\quad 0.91 \%$

Mass Flow Rate

Test Duration, min

Flow Rate, $\mathrm{g} / \mathrm{min}$

\begin{tabular}{|lcccc|}
\hline \multicolumn{5}{ll}{ Test Chamber Environment } \\
\hline Initial Conditions & $\mathrm{RH}(\%)$ & Temperature $\left({ }^{\circ} \mathrm{C}\right)$ & Atm. P. (mbar) \\
1-HUM/TMP-BDST & 90.2 & 24.6 & P-IN-BDST & 860 \\
2-HUM/TMP-BDST & 86.1 & 24.8 & & \\
\hline Final Conditions & $\mathrm{RH}(\%)$ & Temperature $\left({ }^{\circ} \mathrm{C}\right)$ & Atm. P. (mbar) \\
1-HUM/TMP-BDST & 88.9 & 25.7 & P-IN-BDST & 863 \\
2-HUM/TMP-BDST & 85.6 & 25.2 & & \\
\hline
\end{tabular}


Multiple Patch Test Summary and Mass Balance-Smooth DS Surface

Test: $27 \mathrm{~cm}$ left of DS center, 33

Test Date 6/3/02

\begin{tabular}{|c|c|c|c|c|}
\hline & Initial & Final & Difference & $\%$ of Input \\
\hline Water Input & 661.0 & 527.9 & 133.1 & $100 \%$ \\
\hline Evap & 131.8 & 131.8 & 0.0 & \\
\hline $\mathrm{G} 1-1$ & 7.3 & 57.3 & 50.0 & \multirow[b]{5}{*}{$91.4 \%$} \\
\hline G1-2 & 7.2 & 58.9 & 51.7 & \\
\hline G1-3 & 7.4 & 27.0 & 19.6 & \\
\hline G1-4 & 7.3 & 7.6 & 0.3 & \\
\hline SS-1 & & & 0.0 & \\
\hline G2-1 & & & $\overline{0.0}$ & \multirow[b]{5}{*}{$0.0 \%$} \\
\hline G2-2 & & & 0.0 & \\
\hline G2-3 & & & 0.0 & \\
\hline G2-4 & & & 0.0 & \\
\hline SS-2 & & & 0.0 & \\
\hline G3-1 & & & 0.0 & \multirow[b]{5}{*}{$0.0 \%$} \\
\hline G3-2 & & & 0.0 & \\
\hline G3-3 & & & 0.0 & \\
\hline G3-4 & & & 0.0 & \\
\hline SS-3 & & & 0.0 & \\
\hline G4-1 & & & 0.0 & \multirow[b]{5}{*}{$0.0 \%$} \\
\hline G4-2 & & & 0.0 & \\
\hline G4-3 & & & 0.0 & \\
\hline G4-4 & & & 0.0 & \\
\hline SS-4 & & & 0.0 & \\
\hline $\mathrm{B} 1$ & & & $\overline{0.0}$ & $0.0 \%$ \\
\hline B2 & 107.0 & 107.3 & 0.3 & $0.2 \%$ \\
\hline B3 & & & 0.0 & $0.0 \%$ \\
\hline B4 & 106.9 & 107.4 & 0.5 & $0.4 \%$ \\
\hline B5 & & & 0.0 & $0.0 \%$ \\
\hline $\mathrm{B} 6$ & & & 0.0 & $0.0 \%$ \\
\hline DS in-1 & & & 0.0 & \multirow[b]{2}{*}{$0.0 \%$} \\
\hline DS in-2 & & & 0.0 & \\
\hline DS out-1 & 7.2 & 11.7 & 4.5 & \multirow[b]{4}{*}{$4.9 \%$} \\
\hline DS out-2 & 7.2 & 9.2 & 2.0 & \\
\hline DS out-3 & & & 0.0 & \\
\hline DS out- 4 & & & 0.0 & \\
\hline Other & & & 0.0 & $0.0 \%$ \\
\hline
\end{tabular}

Total Input 133.1

Total Collected 128.9

Difference -4.2

$-3 \%$

\section{Evaporation}

Evap Pan Tare Wt, g

Evaporation, g

\begin{tabular}{ll}
\hline$\%$ Evaporation & $0.00 \%$ \\
\hline
\end{tabular}

\begin{tabular}{|lr|}
\hline \multicolumn{2}{|c|}{ Mass Flow Rate } \\
Test Duration, min & 30 \\
Flow Rate, g/min & 4.44 \\
\hline
\end{tabular}

\begin{tabular}{|lcccc|}
\hline \multicolumn{7}{|l|}{ Test Chamber Environment } & & & \\
\hline Initial Conditions & $\mathrm{RH}(\%)$ & Temperature $\left({ }^{\circ} \mathrm{C}\right)$ & & Atm. P. (mbar) \\
1-HUM/TMP-BDST & 91.5 & 22.7 & P-IN-BDST & 868 \\
2-HUM/TMP-BDST & 88.8 & 22.8 & & \\
\hline Final Conditions & $\mathrm{RH}(\%)$ & Temperature $\left({ }^{\circ} \mathrm{C}\right)$ & & Atm. P. (mbar) \\
1-HUM/TMP-BDST & 93.1 & 23.5 & P-IN-BDST & 865 \\
2-HUM/TMP-BDST & 90.7 & 23.6 & & \\
\hline
\end{tabular}


Multiple Patch Test Summary and Mass Balance-Smooth DS Surface

Test: $81 \mathrm{~cm}$ left of DS center, 33

\begin{tabular}{|c|c|c|c|c|}
\hline & Initial & Final & Difference & $\%$ of Input \\
\hline Water Input & 718.1 & 585.7 & 132.4 & $100 \%$ \\
\hline Evap & 136.8 & 136.6 & 0.2 & \\
\hline $\mathrm{G} 1-1$ & 7.1 & 53.0 & 45.9 & \multirow[b]{5}{*}{$84.7 \%$} \\
\hline G1-2 & 7.4 & 60.4 & 53.0 & \\
\hline G1-3 & 7.5 & 20.4 & 12.9 & \\
\hline $\mathrm{G} 1-4$ & 7.4 & 7.7 & 0.3 & \\
\hline SS-1 & & & 0.0 & \\
\hline$\overline{\mathrm{G} 2-1}$ & 7.3 & 7.7 & $\overline{0.4}$ & \multirow[b]{5}{*}{$0.3 \%$} \\
\hline G2-2 & & & 0.0 & \\
\hline G2-3 & & & 0.0 & \\
\hline G2-4 & & & 0.0 & \\
\hline SS-2 & & & 0.0 & \\
\hline G3-1 & & & 0.0 & \multirow[b]{5}{*}{$0.0 \%$} \\
\hline G3-2 & & & 0.0 & \\
\hline G3-3 & & & 0.0 & \\
\hline G3-4 & & & 0.0 & \\
\hline SS-3 & & & 0.0 & \\
\hline$\overline{\mathrm{G} 4-1}$ & & & $\overline{0.0}$ & \multirow[b]{5}{*}{$0.0 \%$} \\
\hline G4-2 & & & 0.0 & \\
\hline G4-3 & & & 0.0 & \\
\hline G4-4 & & & 0.0 & \\
\hline SS-4 & & & 0.0 & \\
\hline $\mathrm{B} 1$ & 107.1 & 107.7 & 0.6 & $0.5 \%$ \\
\hline B2 & & & 0.0 & $0.0 \%$ \\
\hline B3 & & & 0.0 & $0.0 \%$ \\
\hline B4 & 106.7 & 107.4 & 0.7 & $0.5 \%$ \\
\hline B5 & & & 0.0 & $0.0 \%$ \\
\hline $\mathrm{B} 6$ & & & 0.0 & $0.0 \%$ \\
\hline DS in-1 & & & 0.0 & \multirow[b]{2}{*}{$0.0 \%$} \\
\hline DS in-2 & & & 0.0 & \\
\hline DS out-1 & 7.3 & 12.6 & 5.3 & \multirow[b]{4}{*}{$4.8 \%$} \\
\hline DS out-2 & 7.3 & 8.3 & 1.0 & \\
\hline DS out-3 & & & 0.0 & \\
\hline DS out-4 & & & 0.0 & \\
\hline Other & & & 0.0 & $0.0 \%$ \\
\hline
\end{tabular}

Test Date 6/3/02

\begin{tabular}{|l|}
\hline \multicolumn{2}{|c|}{ Total Input 132.4} \\
Total Collected 120.1 \\
\hline \hline \multicolumn{2}{|c|}{$\mathbf{- 9 \%}$} \\
\hline \multicolumn{2}{|c|}{ Difference $\mathbf{- 1 2 . 3}$} \\
\hline \multicolumn{2}{|c|}{ Evaporation } \\
Evap Pan Tare Wt, g \\
Evaporation, g \\
\hline \% Evaporation \\
\hline \multicolumn{2}{|c|}{0.2} \\
\hline \multicolumn{2}{|c|}{ Mass Flow Rate } \\
Test Duration, min \\
Flow Rate, g/min \\
\hline
\end{tabular}

Test Chamber Environment

\begin{tabular}{|lcccc|}
\hline Initial Conditions & $\mathrm{RH}(\%)$ & Temperature $\left({ }^{\circ} \mathrm{C}\right)$ & Atm. P. (mbar) \\
1-HUM/TMP-BDST & 86.6 & 21.4 & P-IN-BDST & 864 \\
2-HUM/TMP-BDST & 88.4 & 21.3 & & \\
\hline Final Conditions & $\mathrm{RH}(\%)$ & Temperature $\left({ }^{\circ} \mathrm{C}\right)$ & & Atm. P. (mbar) \\
1-HUM/TMP-BDST & 91.5 & 22.2 & P-IN-BDST & 862 \\
2-HUM/TMP-BDST & 92.3 & 22.1 & & \\
\hline
\end{tabular}


Bounding Flow Test Summary and Mass Balance-Smooth DS Surface

Test: High flow rate, $54 \mathrm{~cm}$ left of DS center, Crown

\begin{tabular}{|c|c|c|c|c|}
\hline & Initial & Final & Difference & $\%$ of Input \\
\hline Water Input & 853.8 & 516.1 & 337.7 & $100 \%$ \\
\hline Evap & 92.9 & 92.6 & 0.3 & \\
\hline G1-1 & 64.3 & 85.7 & 21.4 & \multirow[b]{5}{*}{$6.3 \%$} \\
\hline G1-2 & & & 0 & \\
\hline G1-3 & & & 0 & \\
\hline G1-4 & & & 0 & \\
\hline SS-1 & & & 0 & \\
\hline G2-1 & 48.5 & 48.9 & 0.4 & \multirow[b]{5}{*}{$0.1 \%$} \\
\hline G2-2 & & & 0 & \\
\hline G2-3 & & & 0 & \\
\hline G2-4 & & & 0 & \\
\hline SS-2 & & & 0 & \\
\hline G3-1 & 56.3 & 152.0 & 95.7 & \multirow[b]{5}{*}{$32.2 \%$} \\
\hline G3-2 & 49.0 & 62.0 & 13 & \\
\hline G3-3 & & & 0 & \\
\hline G3-4 & & & 0 & \\
\hline SS-3 & & & 0 & \\
\hline G4-1 & & & 0 & \multirow[b]{5}{*}{$0.0 \%$} \\
\hline G4-2 & & & 0 & \\
\hline G4-3 & & & 0 & \\
\hline G4-4 & & & 0 & \\
\hline SS-4 & & & 0 & \\
\hline B1 & 107.6 & 108.0 & 0.4 & $0.1 \%$ \\
\hline B2 & 107.2 & 108.2 & 1 & $0.3 \%$ \\
\hline B3 & & & 0 & $0.0 \%$ \\
\hline B4 & 107.3 & 153.7 & 46.4 & $13.7 \%$ \\
\hline B5 & & & 0 & $0.0 \%$ \\
\hline B6 & 110.2 & 112.7 & 2.5 & $0.7 \%$ \\
\hline DS in-1 & & & 0 & \multirow[b]{2}{*}{$0.0 \%$} \\
\hline DS in-2 & & & 0 & \\
\hline DS out-1 & 56.7 & 146.1 & 89.4 & \multirow[b]{4}{*}{$34.0 \%$} \\
\hline DS out-2 & 55.5 & 81.0 & 25.5 & \\
\hline DS out-3 & & & 0 & \\
\hline DS out-4 & & & 0 & \\
\hline Other & & & 0 & $0.0 \%$ \\
\hline
\end{tabular}

Test Date $\quad 6 / 18 / 02$

\begin{tabular}{|c|}
\hline Tetal Input 337.7 \\
Total Collected 295.7 \\
\hline \hline Difference -42 \\
$-12 \%$ \\
\hline
\end{tabular}

\begin{tabular}{|lr|}
\hline \multicolumn{2}{|c|}{ Evaporation } \\
Evap Pan Tare Wt, g & 66.7 \\
Evaporation, g & 0.3 \\
\hline \% Evaporation & $\mathbf{1 . 1 5 \%}$ \\
\hline
\end{tabular}

\begin{tabular}{|lr|}
\hline \multicolumn{2}{|c|}{ Mass Flow Rate } \\
Test Duration, min & 10.02 \\
Flow Rate, g/min & 33.71 \\
\hline
\end{tabular}

\begin{tabular}{|c|c|c|c|c|}
\hline \multicolumn{5}{|c|}{ Test Chamber Environment } \\
\hline Initial Conditions & $\mathrm{RH}(\%)$ & Temperature $\left({ }^{\circ} \mathrm{C}\right)$ & & Atm. P. (mbar) \\
\hline 1-HUM/TMP-BDST & 97.6 & 30.6 & P-IN-BDST & 861 \\
\hline 2-HUM/TMP-BDST & 89.7 & 30.4 & & \\
\hline Final Conditions & $\mathrm{RH}(\%)$ & Temperature $\left({ }^{\circ} \mathrm{C}\right)$ & & Atm. P. (mbar) \\
\hline 1-HUM/TMP-BDST & 99.4 & 30.9 & P-IN-BDST & 860 \\
\hline 2-HUM/TMP-BDST & 94.2 & 30.4 & & \\
\hline
\end{tabular}


Bounding Flow Test Summary and Mass Balance-Smooth DS Surface

Test: Low flow rate, $54 \mathrm{~cm}$ left of DS Center, Crown

\begin{tabular}{|c|c|c|c|c|c|c|}
\hline \multicolumn{5}{|c|}{ Test: Low flow rate, $54 \mathrm{~cm}$ left of DS Center, Crown } & Test Date & 6/18/02 \\
\hline & Initial & Final & Difference & $\%$ of Input & \multicolumn{2}{|c|}{ Total Input 88.9} \\
\hline Water Input & 769.2 & 680.3 & 88.9 & $100 \%$ & \multicolumn{2}{|c|}{ Total Collected 67.3} \\
\hline Evap & 94.5 & 93.3 & 1.2 & & \multirow{2}{*}{\multicolumn{2}{|c|}{$\begin{array}{r}-21.6 \\
-24 \%\end{array}$}} \\
\hline G1-1 & 57.0 & 58.5 & 1.5 & \multirow[b]{5}{*}{$1.7 \%$} & & \\
\hline G1-2 & & & 0 & & & \\
\hline G1-3 & & & 0 & & \multicolumn{2}{|c|}{ Evaporation } \\
\hline G1-4 & & & 0 & & \multirow{2}{*}{$\begin{array}{l}\text { Evap Pan Tare Wt, g } \\
\text { Evaporation, g }\end{array}$} & \multirow{2}{*}{$\begin{array}{r}66.7 \\
1.2\end{array}$} \\
\hline SS-1 & & & 0 & & & \\
\hline G2-1 & 41.0 & 41.0 & 0 & \multirow[b]{5}{*}{$0.0 \%$} & \multirow[t]{2}{*}{ \% Evaporation } & \multirow[t]{2}{*}{$4.32 \%$} \\
\hline G2-2 & & & 0 & & & \\
\hline G2-3 & & & 0 & & & \\
\hline G2-4 & & & 0 & & \multicolumn{2}{|c|}{ Mass Flow Rate } \\
\hline SS-2 & & & 0 & & \multirow{3}{*}{$\begin{array}{l}\text { Test Duration, } \min \\
\text { Flow Rate, g/min }\end{array}$} & \multirow{3}{*}{$\begin{array}{r}300.03 \\
0.30\end{array}$} \\
\hline G3-1 & 63.5 & 73.5 & 10 & & & \\
\hline G3-2 & & & 0 & & & \\
\hline G3-3 & & & 0 & & & \\
\hline
\end{tabular}

\begin{tabular}{|c|c|c|c|c|}
\hline G3-3 & & & 0 & \multirow[b]{3}{*}{$11.2 \%$} \\
\hline G3-4 & & & 0 & \\
\hline SS-3 & & & 0 & \\
\hline G4-1 & & & 0 & \multirow[b]{5}{*}{$0.0 \%$} \\
\hline G4-2 & & & 0 & \\
\hline G4-3 & & & 0 & \\
\hline G4-4 & & & 0 & \\
\hline SS-4 & & & 0 & \\
\hline$\overline{B 1}$ & & & 0 & $0.0 \%$ \\
\hline $\mathrm{B} 2$ & 8.1 & 8.7 & 0.6 & $0.7 \%$ \\
\hline B3 & & & 0 & $0.0 \%$ \\
\hline B4 & 107.7 & 115.6 & 7.9 & $8.9 \%$ \\
\hline B5 & & & 0 & $0.0 \%$ \\
\hline $\mathrm{B} 6$ & 110.2 & 110.4 & 0.2 & $0.2 \%$ \\
\hline DS in-1 & & & 0 & \multirow[b]{2}{*}{$0.0 \%$} \\
\hline DS in-2 & & & 0 & \\
\hline DS out-1 & 63.9 & 105.1 & 41.2 & \multirow[b]{4}{*}{$46.3 \%$} \\
\hline DS out-2 & & & 0 & \\
\hline DS out-3 & & & 0 & \\
\hline DS out-4 & & & 0 & \\
\hline Other & 8.2 & 14.1 & 5.9 & $6.6 \%$ \\
\hline \multicolumn{3}{|c|}{ Total Collecte } & 67.3 & $75.7 \%$ \\
\hline
\end{tabular}

\begin{tabular}{|lcccc|}
\hline \multicolumn{4}{l}{ Test Chamber Environment } \\
\hline Initial Conditions & $\mathrm{RH}(\%)$ & Temperature $\left({ }^{\circ} \mathrm{C}\right)$ & Atm. P. (mbar) \\
1-HUM/TMP-BDST & 94.1 & 26.4 & P-IN-BDST & 864 \\
2-HUM/TMP-BDST & 94.8 & 26.1 & & \\
\hline Final Conditions & $\mathrm{RH}(\%)$ & Temperature $\left({ }^{\circ} \mathrm{C}\right)$ & Atm. P. (mbar) \\
1-HUM/TMP-BDST & 107.2 & 30.4 & P-IN-BDST & 861 \\
2-HUM/TMP-BDST & 93.2 & 30.3 & & \\
\hline
\end{tabular}


Bounding Flow Test Summary and Mass Balance-Smooth DS Surface

Test: High flow rate, $27 \mathrm{~cm}$ left of DS center, Crown

\begin{tabular}{|c|c|c|c|c|}
\hline & Initial & Final & Difference & $\%$ of Input \\
\hline Water Input & 857.5 & 524.8 & 332.7 & $100 \%$ \\
\hline Evap & 94.9 & 94.5 & 0.4 & \\
\hline G1-1 & 56.7 & 82.8 & 26.1 & \multirow[b]{5}{*}{$8.7 \%$} \\
\hline $\mathrm{G} 1-2$ & 48.1 & 51.1 & 3 & \\
\hline G1-3 & & & 0 & \\
\hline G1-4 & & & 0 & \\
\hline SS-1 & & & 0 & \\
\hline G2-1 & 40.9 & 41.0 & 0.1 & \multirow[b]{5}{*}{$0.0 \%$} \\
\hline G2-2 & & & 0 & \\
\hline G2-3 & & & 0 & \\
\hline G2-4 & & & 0 & \\
\hline SS-2 & & & 0 & \\
\hline G3-1 & 55.9 & 157.5 & 101.6 & \multirow[b]{5}{*}{$38.4 \%$} \\
\hline G3-2 & 56.1 & 82.1 & 26 & \\
\hline G3-3 & & & 0 & \\
\hline G3-4 & & & 0 & \\
\hline SS-3 & & & 0 & \\
\hline G4-1 & & & 0 & \multirow[b]{5}{*}{$0.0 \%$} \\
\hline G4-2 & & & 0 & \\
\hline G4-3 & & & 0 & \\
\hline G4-4 & & & 0 & \\
\hline SS-4 & & & 0 & \\
\hline$B 1$ & & & 0 & $0.0 \%$ \\
\hline $\mathrm{B} 2$ & 106.7 & 121.1 & 14.4 & $4.3 \%$ \\
\hline B3 & & & 0 & $0.0 \%$ \\
\hline B4 & 107.2 & 110.5 & 3.3 & $1.0 \%$ \\
\hline B5 & 7.6 & 7.9 & 0.3 & $0.1 \%$ \\
\hline B6 & 109.8 & 121.7 & 11.9 & $3.6 \%$ \\
\hline DS in-1 & & & 0 & \multirow[b]{2}{*}{$0.0 \%$} \\
\hline DS in-2 & & & 0 & \\
\hline DS out-1 & 56.3 & 147.2 & 90.9 & \multirow[b]{4}{*}{$33.9 \%$} \\
\hline DS out-2 & 55.1 & 76.9 & 21.8 & \\
\hline DS out-3 & & & 0 & \\
\hline DS out-4 & & & 0 & \\
\hline Other & & & 0 & $0.0 \%$ \\
\hline
\end{tabular}

Test Date $\quad 6 / 18 / 02$

\begin{tabular}{|r|}
\hline Total Input 332.7 \\
Total Collected 299.4 \\
\hline \hline Difference $\mathbf{- 3 3 . 3}$ \\
$-\mathbf{- 1 0 \%}$ \\
\hline
\end{tabular}

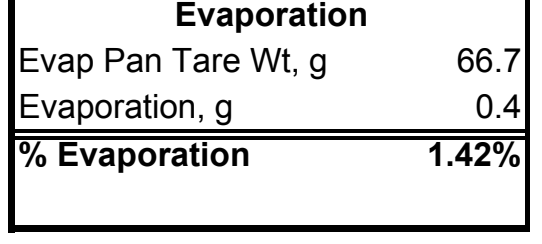

\begin{tabular}{|lr|}
\hline \multicolumn{2}{|c|}{ Mass Flow Rate } \\
Test Duration, min & 10 \\
Flow Rate, g/min & 33.27 \\
\hline
\end{tabular}

Test Chamber Environment

\begin{tabular}{|lcccc|}
\hline Initial Conditions & $\mathrm{RH}(\%)$ & Temperature $\left({ }^{\circ} \mathrm{C}\right)$ & & Atm. P. (mbar) \\
1-HUM/TMP-BDST & 88.1 & 24.9 & P-IN-BDST & 858 \\
2-HUM/TMP-BDST & 91.8 & 24.6 & & \\
\hline Final Conditions & $\mathrm{RH}(\%)$ & Temperature $\left({ }^{\circ} \mathrm{C}\right)$ & & Atm. P. (mbar) \\
1-HUM/TMP-BDST & 93 & 26.1 & P-IN-BDST & 860 \\
2-HUM/TMP-BDST & 94.7 & 25.7 & & \\
\hline
\end{tabular}


Bounding Flow Test Summary and Mass Balance-Smooth DS Surface

Test: Low flow rate, $27 \mathrm{~cm}$ left of DS Center, Crown

\begin{tabular}{|c|c|c|c|c|}
\hline & Initial & Final & Difference & $\%$ of Input \\
\hline$\overline{\text { Water Input }}$ & 872.2 & $\overline{771.2}$ & 101 & $100 \%$ \\
\hline Evap & 93.2 & 91.8 & 1.4 & \\
\hline $\mathrm{G} 1-1$ & 42.6 & 48.1 & 5.5 & \multirow[b]{5}{*}{$5.4 \%$} \\
\hline$\overline{\mathrm{G} 1-2}$ & & & 0 & \\
\hline G1-3 & & & 0 & \\
\hline$\overline{G 1-4}$ & & & 0 & \\
\hline SS-1 & & & 0 & \\
\hline G2-1 & & & 0 & \multirow[b]{5}{*}{$0.0 \%$} \\
\hline G2-2 & & & 0 & \\
\hline G2-3 & & & 0 & \\
\hline $\bar{G} 2-4$ & & & 0 & \\
\hline SS-2 & & & 0 & \\
\hline$\overline{\mathrm{G} 3-1}$ & 57.6 & 69.3 & 11.7 & \multirow[b]{5}{*}{$11.6 \%$} \\
\hline G3-2 & & & 0 & \\
\hline G3-3 & & & 0 & \\
\hline G3-4 & & & 0 & \\
\hline SS-3 & & & 0 & \\
\hline G4-1 & & & 0 & \multirow[b]{5}{*}{$0.0 \%$} \\
\hline G4-2 & & & 0 & \\
\hline G4-3 & & & 0 & \\
\hline G4-4 & & & 0 & \\
\hline SS-4 & & & 0 & \\
\hline$\overline{\mathrm{B} 1}$ & & & 0 & $0.0 \%$ \\
\hline B2 & 106.4 & 107.3 & 0.9 & $0.9 \%$ \\
\hline B3 & & & 0 & $0.0 \%$ \\
\hline B4 & 107.0 & 107.6 & 0.6 & $0.6 \%$ \\
\hline B5 & & & 0 & $0.0 \%$ \\
\hline $\mathrm{B} 6$ & 109.6 & 110.7 & 1.1 & $1.1 \%$ \\
\hline$\overline{D S}$ in-1 & & & 0 & \multirow[b]{2}{*}{$0.0 \%$} \\
\hline$\overline{D S}$ in-2 & & & 0 & \\
\hline DS out- 1 & 64.0 & 99.8 & 35.8 & \multirow[b]{4}{*}{$35.4 \%$} \\
\hline DS out-2 & & & 0 & \\
\hline DS out-3 & & & 0 & \\
\hline DS out-4 & & & 0 & \\
\hline Other & & & 0 & $0.0 \%$ \\
\hline
\end{tabular}

Test Date $\quad 6 / 17 / 02$

\begin{tabular}{|r|}
\hline Total Input 101.0 \\
Total Collected 55.6 \\
\hline \hline Difference $\mathbf{- 4 5 . 4}$ \\
$\mathbf{- 4 5 \%}$ \\
\hline
\end{tabular}

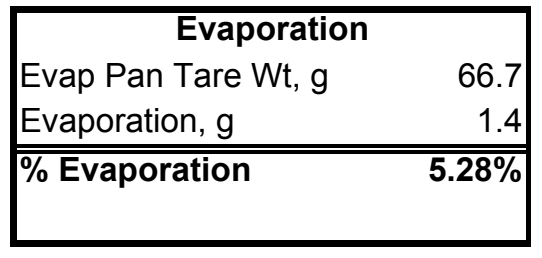

\begin{tabular}{|lr|}
\hline \multicolumn{2}{|c|}{ Mass Flow Rate } \\
Test Duration, min & 300 \\
Flow Rate, g/min & 0.34 \\
\hline
\end{tabular}

\begin{tabular}{|lcccc|}
\hline \multicolumn{7}{l}{ Test Chamber Environment } \\
\hline Initial Conditions & $\mathrm{RH}(\%)$ & Temperature $\left({ }^{\circ} \mathrm{C}\right)$ & & Atm. P. (mbar) \\
1-HUM/TMP-BDST & 95.1 & 28.5 & P-IN-BDST & 863 \\
2-HUM/TMP-BDST & 91.5 & 28.5 & & \\
\hline Final Conditions & $\mathrm{RH}(\%)$ & Temperature $\left({ }^{\circ} \mathrm{C}\right)$ & & Atm. P. (mbar) \\
1-HUM/TMP-BDST & 94.4 & 30.3 & P-IN-BDST & 874 \\
2-HUM/TMP-BDST & 94.8 & 29.7 & & \\
\hline
\end{tabular}


Bounding Flow Test Summary and Mass Balance-Smooth DS Surface

Test: High flow rate, $27 \mathrm{~cm}$ right of DS center, Crown

Test Date $\quad 6 / 17 / 02$

\begin{tabular}{|c|c|c|c|c|c|}
\hline & Initial & Final & Difference & $\%$ of Input & Total Input 378.2 \\
\hline Water Input & 907.3 & (529.1 & 378.2 & $\overline{100 \%}$ & Total Collected 350.5 \\
\hline Evap & 93.5 & 93.5 & 0 & & "Difference -27.7 \\
\hline G1-1 & 64.4 & 124.4 & 60 & & $-7 \%$ \\
\hline G1-2 & & & 0 & & \\
\hline
\end{tabular}

\begin{tabular}{|l|l|l|l|}
\hline G1-3 & & & 0 \\
\hline G1-4 & & & 0 \\
\hline
\end{tabular}

\begin{tabular}{|l|l|l|r}
\hline G1-4 & & & 0 \\
\hline SS-1 & & & \\
\hline
\end{tabular}

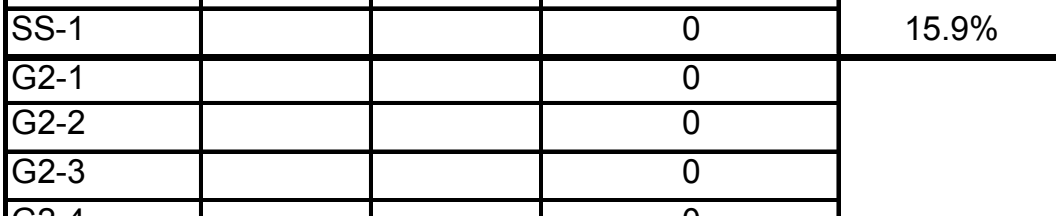

G2-4

SS-2

G3-1

G3-2

G3-3

G3-4

SS-3

G4-1

G4-2

G4-3

G4-4

SS-4

$\mathrm{B} 1$

$\mathrm{B} 2$

B3

B4

B5

\begin{tabular}{|c|c|c|c|c|}
\hline B6 & 110.3 & 124.3 & 14 & $3.7 \%$ \\
\hline DS in-1 & & & 0 & \multirow[b]{2}{*}{$0.0 \%$} \\
\hline DS in-2 & & & 0 & \\
\hline DS out-1 & 63.5 & 175.9 & 112.4 & \multirow[b]{4}{*}{$35.6 \%$} \\
\hline DS out- 2 & 48.0 & 70.1 & 22.1 & \\
\hline DS out-3 & & & 0 & \\
\hline DS out-4 & & & 0 & \\
\hline Other & & & 0 & $0.0 \%$ \\
\hline
\end{tabular}

Evap Pan Tare Wt, g

Evaporation, $\mathrm{g}$

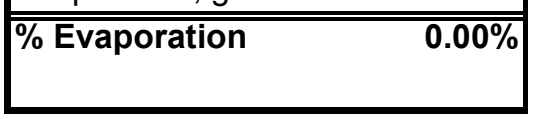

\begin{tabular}{|lr|}
\hline \multicolumn{2}{|c|}{ Mass Flow Rate } \\
Test Duration, min & 10 \\
Flow Rate, g/min & 37.82 \\
\hline
\end{tabular}

$36.1 \%$

\begin{tabular}{|lcccc|}
\hline \multicolumn{7}{l}{ Test Chamber Environment } & & & \\
\hline Initial Conditions & $\mathrm{RH}(\%)$ & Temperature $\left({ }^{\circ} \mathrm{C}\right)$ & & Atm. P. (mbar) \\
1-HUM/TMP-BDST & 94.4 & 27.1 & P-IN-BDST & 858 \\
2-HUM/TMP-BDST & 92.7 & 27.1 & & \\
\hline Final Conditions & $\mathrm{RH}(\%)$ & Temperature $\left({ }^{\circ} \mathrm{C}\right)$ & & Atm. P. (mbar) \\
1-HUM/TMP-BDST & 96 & 28.2 & P-IN-BDST & 863 \\
2-HUM/TMP-BDST & 94.5 & 28 & & \\
\hline
\end{tabular}

SN-M\&O-SCl-043-V2, pages 36-37 
Bounding Flow Test Summary and Mass Balance-Smooth DS Surface

Test: low flow, $27 \mathrm{~cm}$ right of DS center, Crown

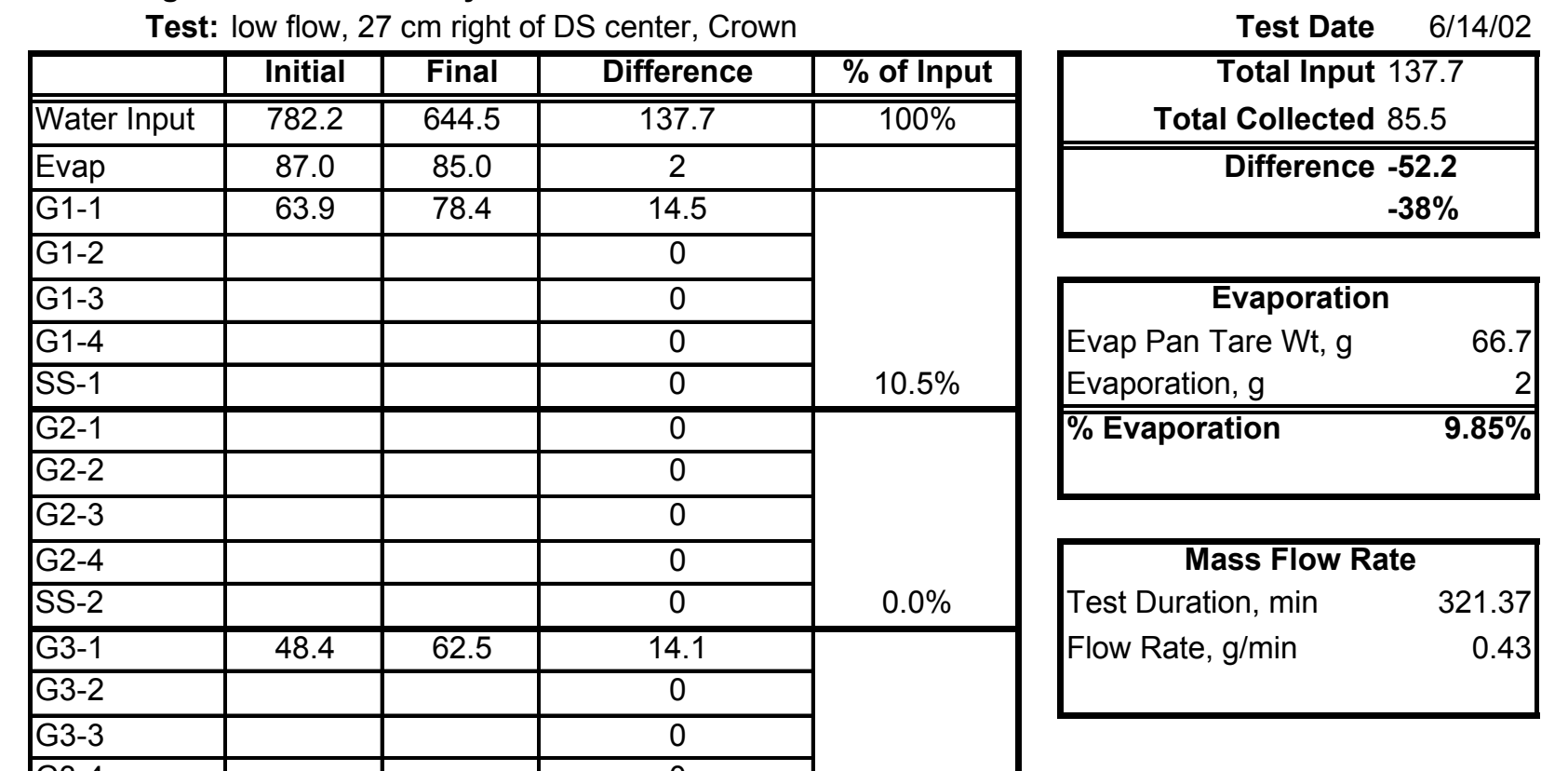

\begin{tabular}{|c|c|c|c|c|}
\hline & & & & \multirow[b]{3}{*}{$10.2 \%$} \\
\hline G3-4 & & & 0 & \\
\hline SS-3 & & & 0 & \\
\hline$\overline{\mathrm{G} 4-1}$ & & & 0 & \multirow[b]{5}{*}{$0.0 \%$} \\
\hline G4-2 & & & 0 & \\
\hline G4-3 & & & 0 & \\
\hline G4-4 & & & 0 & \\
\hline SS-4 & & & 0 & \\
\hline $\mathrm{B} 1$ & & & 0 & $0.0 \%$ \\
\hline $\mathrm{B} 2$ & 107.1 & 108.5 & 1.4 & $1.0 \%$ \\
\hline B3 & & & 0 & $0.0 \%$ \\
\hline B4 & 107.6 & 107.8 & 0.2 & $0.1 \%$ \\
\hline B5 & 109.5 & 114.0 & 4.5 & $3.3 \%$ \\
\hline B6 & 110.2 & 111.8 & 1.6 & $1.2 \%$ \\
\hline$\overline{D S}$ in-1 & & & 0 & \multirow[b]{2}{*}{$0.0 \%$} \\
\hline$\overline{D S}$ in-2 & & & 0 & \\
\hline DS out-1 & 64.1 & 113.3 & 49.2 & \multirow[b]{4}{*}{$35.7 \%$} \\
\hline DS out-2 & & & 0 & \\
\hline DS out-3 & & & 0 & \\
\hline DS out-4 & & & 0 & \\
\hline Other & & & 0 & $0.0 \%$ \\
\hline
\end{tabular}

\begin{tabular}{|lcccc|}
\hline Test Chamber Environment & \multicolumn{3}{l|}{} \\
\hline Initial Conditions & $\mathrm{RH}(\%)$ & Temperature $\left({ }^{\circ} \mathrm{C}\right)$ & Atm. P. (mbar) \\
1-HUM/TMP-BDST & 91.3 & 24.4 & P-IN-BDST & 875 \\
2-HUM/TMP-BDST & 91.8 & 24.1 & & \\
\hline Final Conditions & $\mathrm{RH}(\%)$ & Temperature $\left({ }^{\circ} \mathrm{C}\right)$ & & Atm. P. (mbar) \\
1-HUM/TMP-BDST & 99.3 & 28.9 & P-IN-BDST & 873 \\
2-HUM/TMP-BDST & 93.1 & 28.8 & & \\
\hline
\end{tabular}


Bounding Flow Test Summary and Mass Balance-Smooth DS Surface

Test: High flow rate, $54 \mathrm{~cm}$ left of DS center, 16.5

\begin{tabular}{|c|c|c|c|c|}
\hline & Initial & Final & Difference & $\%$ of Input \\
\hline Water Input & 850.0 & $\overline{496.6}$ & 353.4 & $100 \%$ \\
\hline Evap & 92.1 & 91.8 & 0.3 & \\
\hline$\overline{\mathrm{G} 1-1}$ & 57.2 & 74.6 & 17.4 & \multirow[b]{5}{*}{$8.9 \%$} \\
\hline$\overline{\mathrm{G} 1-2}$ & 7.4 & 21.5 & 14.1 & \\
\hline$\overline{G 1-3}$ & & & 0 & \\
\hline G1-4 & & & 0 & \\
\hline SS-1 & & & 0 & \\
\hline G2-1 & 48.5 & 49.8 & 1.3 & \multirow[b]{5}{*}{$0.4 \%$} \\
\hline $\bar{G} 2-2$ & & & 0 & \\
\hline$\overline{\mathrm{G} 2-3}$ & & & 0 & \\
\hline G2-4 & & & 0 & \\
\hline SS-2 & & & 0 & \\
\hline G3-1 & 49.1 & 50.0 & 0.9 & \multirow[b]{5}{*}{$0.3 \%$} \\
\hline G3-2 & & & 0 & \\
\hline G3-3 & & & 0 & \\
\hline G3-4 & & & 0 & \\
\hline SS-3 & & & 0 & \\
\hline G4-1 & & & 0 & \multirow[b]{5}{*}{$0.0 \%$} \\
\hline G4-2 & & & 0 & \\
\hline G4-3 & & & 0 & \\
\hline G4-4 & & & 0 & \\
\hline SS-4 & & & 0 & \\
\hline $\mathrm{B} 1$ & 107.9 & 108.3 & 0.4 & $0.1 \%$ \\
\hline $\mathrm{B} 2$ & 108.0 & 108.4 & 0.4 & $0.1 \%$ \\
\hline B3 & & & 0 & $0.0 \%$ \\
\hline B4 & 107.4 & 277.2 & 169.8 & $48.0 \%$ \\
\hline B5 & & & 0 & $0.0 \%$ \\
\hline $\bar{B} 6$ & 109.7 & 110.1 & 0.4 & $0.1 \%$ \\
\hline$\overline{D S}$ in-1 & & & 0 & \multirow[b]{2}{*}{$0.0 \%$} \\
\hline DS in-2 & & & 0 & \\
\hline DS out-1 & 64.2 & 134.7 & 70.5 & \multirow[b]{4}{*}{$30.6 \%$} \\
\hline DS out-2 & 48.3 & 84.5 & 36.2 & \\
\hline DS out-3 & 7.6 & 9.0 & 1.4 & \\
\hline DS out-4 & & & 0 & \\
\hline Other & & & 0 & $0.0 \%$ \\
\hline
\end{tabular}

Test Date $\quad 6 / 18 / 02$
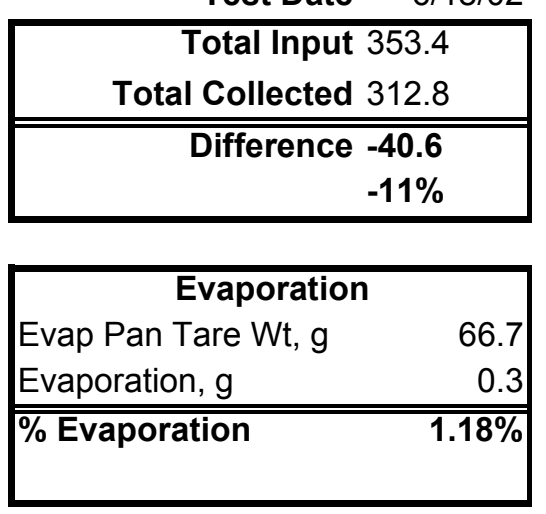

\begin{tabular}{|ll|}
\hline \multicolumn{2}{|c|}{ Mass Flow Rate } \\
Test Duration, $\min$ & 10.15 \\
Flow Rate, $\mathrm{g} / \mathrm{min}$ & 34.82 \\
\hline
\end{tabular}

\begin{tabular}{|lcccc|}
\hline \multicolumn{7}{l}{ Test Chamber Environment } & \multicolumn{3}{l|}{} \\
\hline Initial Conditions & $\mathrm{RH}(\%)$ & Temperature $\left({ }^{\circ} \mathrm{C}\right)$ & Atm. P. (mbar) \\
1-HUM/TMP-BDST & 96.2 & 30.8 & P-IN-BDST & 859 \\
2-HUM/TMP-BDST & 91.8 & 30.4 & & \\
\hline Final Conditions & $\mathrm{RH}(\%)$ & Temperature $\left({ }^{\circ} \mathrm{C}\right)$ & & Atm. P. (mbar) \\
1-HUM/TMP-BDST & 99 & 31 & P-IN-BDST & 864 \\
2-HUM/TMP-BDST & 94.3 & 30.6 & & \\
\hline
\end{tabular}


Bounding Flow Test Summary and Mass Balance-Smooth DS Surface

Test: Low flow rate, $54 \mathrm{~cm}$ left of DS Center, 16.5

\begin{tabular}{|c|c|c|c|c|}
\hline & Initial & Final & Difference & $\%$ of Input \\
\hline Water Input & 822.7 & $\overline{7715.7}$ & 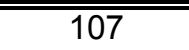 & $\overline{~ 100 \%}$ \\
\hline Evap & 109.1 & 109.8 & -0.7 & \\
\hline $\mathrm{G} 1-1$ & 57.1 & 58.1 & 1 & \multirow[b]{5}{*}{$0.9 \%$} \\
\hline$\overline{\mathrm{G} 1-2}$ & & & 0 & \\
\hline G1-3 & & & 0 & \\
\hline $\mathrm{G} 1-4$ & & & 0 & \\
\hline SS-1 & & & 0 & \\
\hline$\overline{\mathrm{G} 2-1}$ & 41.0 & 41.0 & $\overline{0}$ & \multirow[b]{5}{*}{$0.0 \%$} \\
\hline G2-2 & & & 0 & \\
\hline G2-3 & & & 0 & \\
\hline G2-4 & & & 0 & \\
\hline SS-2 & & & 0 & \\
\hline G3-1 & 41.5 & 41.6 & 0.1 & \multirow[b]{5}{*}{$0.1 \%$} \\
\hline G3-2 & & & 0 & \\
\hline G3-3 & & & 0 & \\
\hline G3-4 & & & 0 & \\
\hline SS-3 & & & 0 & \\
\hline G4-1 & & & 0 & \multirow[b]{5}{*}{$0.0 \%$} \\
\hline G4-2 & & & 0 & \\
\hline G4-3 & & & 0 & \\
\hline G4-4 & & & 0 & \\
\hline SS-4 & & & 0 & \\
\hline $\mathrm{B} 1$ & 106.6 & 107.5 & 0.9 & $0.8 \%$ \\
\hline B2 & 107.4 & 108.6 & 1.2 & $1.1 \%$ \\
\hline B3 & & & 0 & $0.0 \%$ \\
\hline B4 & 107.7 & 192.2 & 84.5 & $79.0 \%$ \\
\hline B5 & & & 0 & $0.0 \%$ \\
\hline B6 & 109.7 & 110.6 & 0.9 & $0.8 \%$ \\
\hline DS in-1 & & & 0 & \multirow[b]{2}{*}{$0.0 \%$} \\
\hline DS in-2 & & & 0 & \\
\hline DS out-1 & 64.4 & 72.1 & 7.7 & \multirow[b]{4}{*}{$7.2 \%$} \\
\hline DS out-2 & & & 0 & \\
\hline DS out-3 & & & 0 & \\
\hline DS out-4 & & & 0 & \\
\hline Other & & & $\overline{0}$ & $0.0 \%$ \\
\hline
\end{tabular}

Test Date 6/19/02

\begin{tabular}{|r|}
\hline Total Input 107.0 \\
Total Collected 96.3 \\
\hline \hline Difference -10.7 \\
$-10 \%$ \\
\hline
\end{tabular}

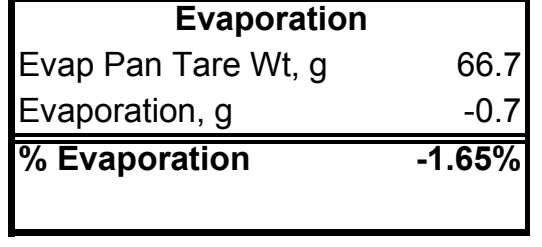

\begin{tabular}{|lr|}
\hline \multicolumn{2}{|c|}{ Mass Flow Rate } \\
Test Duration, min & 300 \\
Flow Rate, g/min & 0.36 \\
\hline
\end{tabular}

Test Chamber Environment

\begin{tabular}{|lcccc|}
\hline Initial Conditions & $\mathrm{RH}(\%)$ & Temperature $\left({ }^{\circ} \mathrm{C}\right)$ & & Atm. P. (mbar) \\
1-HUM/TMP-BDST & 89 & 25.7 & P-IN-BDST & 867 \\
2-HUM/TMP-BDST & 92.4 & 25.4 & & \\
\hline Final Conditions & $\mathrm{RH}(\%)$ & Temperature $\left({ }^{\circ} \mathrm{C}\right)$ & & Atm. P. (mbar) \\
1-HUM/TMP-BDST & 103.5 & 29.8 & P-IN-BDST & 862 \\
2-HUM/TMP-BDST & 94.4 & 29.7 & & \\
\hline
\end{tabular}


Bounding Flow Test Summary and Mass Balance-Smooth DS Surface

Test: High flow rate, $27 \mathrm{~cm}$ left of DS Center, 16.5

Test Date 6/20/02

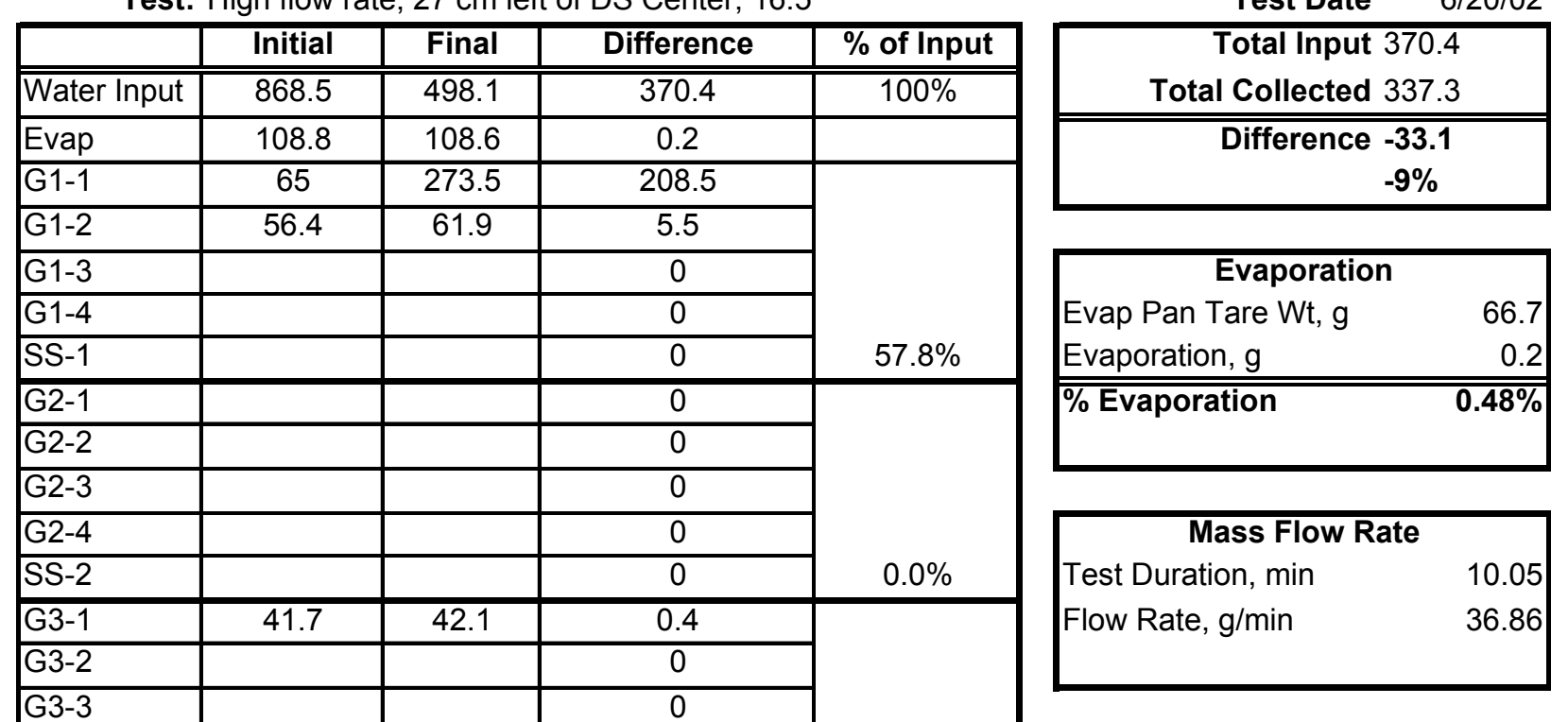

G3-4

SS-3

G4-1

G4-2

G4-3

G4-4

SS-4

B1

B2

\begin{tabular}{|l|c|c|r|}
\hline B3 & 107.5 & 113.1 & 5.6 \\
\hline B4 & & & 0 \\
\hline B5 & 107.2 & 110.6 & 3.4 \\
\hline
\end{tabular}

\begin{tabular}{|c|c|c|c|c|}
\hline B5 & 109 & 110.2 & 1.2 & $0.3 \%$ \\
\hline $\mathrm{B} 6$ & & & 0 & $0.0 \%$ \\
\hline DS in-1 & & & 0 & \multirow[b]{2}{*}{$0.0 \%$} \\
\hline$\overline{D S}$ in-2 & & & 0 & \\
\hline DS out-1 & 64.7 & 149 & 84.3 & \multirow[b]{4}{*}{$30.4 \%$} \\
\hline DS out-2 & 55.9 & 84.3 & 28.4 & \\
\hline DS out-3 & & & 0 & \\
\hline DS out-4 & & & 0 & \\
\hline Other & & & 0 & $0.0 \%$ \\
\hline
\end{tabular}

\begin{tabular}{|lcccc|}
\hline Test Chamber Environment & & & \\
\hline Initial Conditions & $\mathrm{RH}(\%)$ & Temperature $\left({ }^{\circ} \mathrm{C}\right)$ & & Atm. P. (mbar) \\
1-HUM/TMP-BDST & 82.6 & 26.6 & P-IN-BDST & 864 \\
2-HUM/TMP-BDST & 88.4 & 26.3 & & \\
\hline Final Conditions & $\mathrm{RH}(\%)$ & Temperature $\left({ }^{\circ} \mathrm{C}\right)$ & & Atm. P. (mbar) \\
1-HUM/TMP-BDST & 92 & 27.4 & P-IN-BDST & 866 \\
2-HUM/TMP-BDST & 94.2 & 27.2 & & \\
\hline
\end{tabular}


Bounding Flow Test Summary and Mass Balance-Smooth DS Surface

Test: Low flow rate, $27 \mathrm{~cm}$ left of DS Center, 16.5

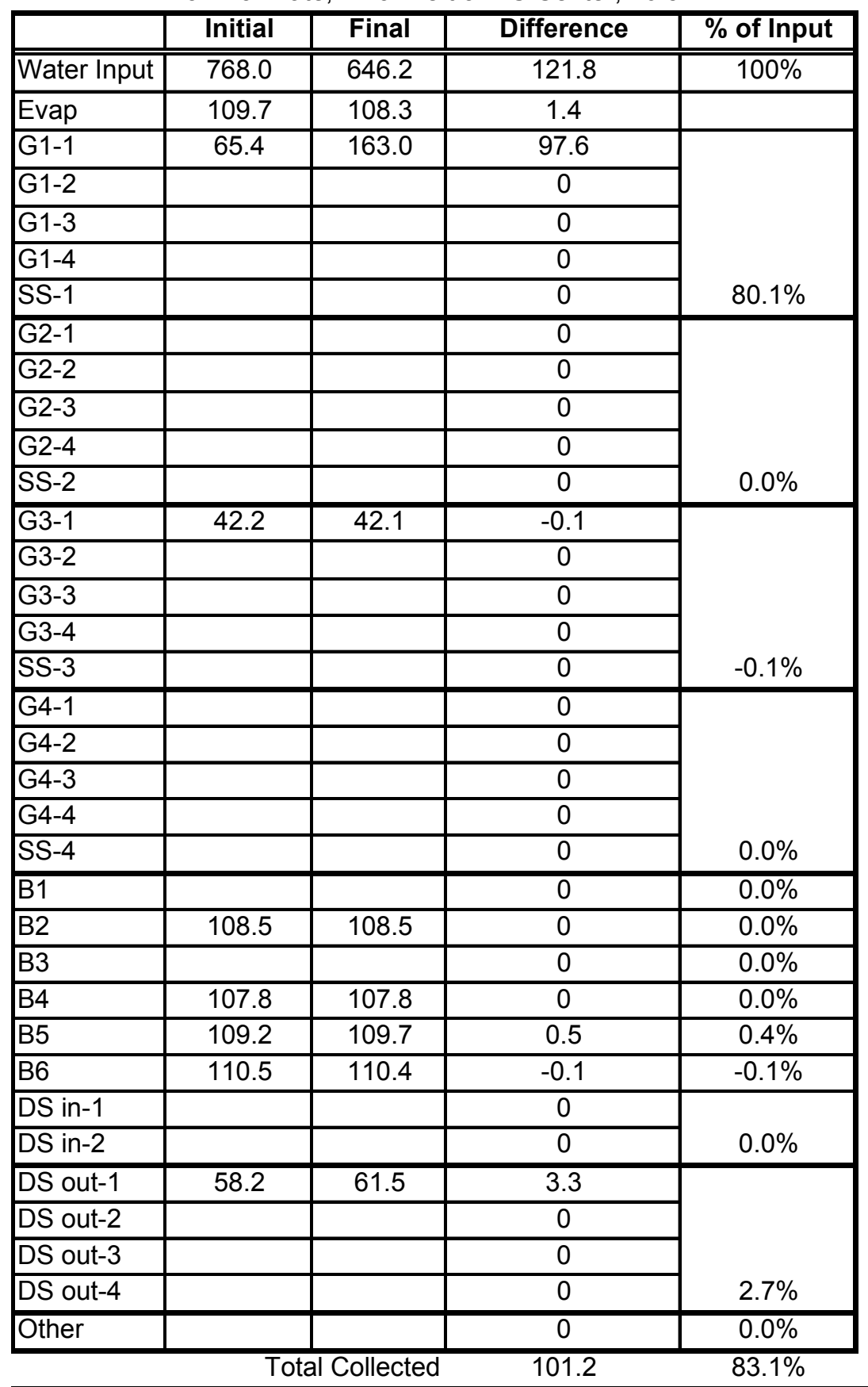

Test Date 6/20/02

\begin{tabular}{|l|}
\hline \multicolumn{2}{|c|}{ Total Input 121.8} \\
Total Collected 101.2 \\
\hline \multicolumn{3}{|c|}{ Difference $\mathbf{- 2 0 . 6}$} \\
\multicolumn{2}{|c|}{$\mathbf{- 1 7 \%}$} \\
\hline \multicolumn{2}{|c|}{ Evaporation } \\
Evap Pan Tare Wt, g \\
Evaporation, g \\
\hline \% Evaporation \\
\hline \multicolumn{2}{|c|}{1.4} \\
\hline \multicolumn{2}{|c|}{$\mathbf{3 . 2 6 \%}$} \\
\hline Mass Flow Rate \\
Test Duration, min \\
Flow Rate, g/min \\
\hline
\end{tabular}

\begin{tabular}{|lcccc|}
\hline \multicolumn{4}{l}{ Test Chamber Environment } & \multicolumn{3}{l|}{} \\
\hline Initial Conditions & $\mathrm{RH}(\%)$ & Temperature $\left({ }^{\circ} \mathrm{C}\right)$ & Atm. P. (mbar) \\
1-HUM/TMP-BDST & 103.1 & 30 & P-IN-BDST & 863 \\
2-HUM/TMP-BDST & 93.8 & 29.9 & & \\
\hline Final Conditions & $\mathrm{RH}(\%)$ & Temperature $\left({ }^{\circ} \mathrm{C}\right)$ & & Atm. P. (mbar) \\
1-HUM/TMP-BDST & 100.9 & 31 & P-IN-BDST & 877 \\
2-HUM/TMP-BDST & 93.8 & 30.7 & & \\
\hline
\end{tabular}


Bounding Flow Test Summary and Mass Balance-Smooth DS Surface

Test: High flow rate, $27 \mathrm{~cm}$ right of DS center, 16.5

\begin{tabular}{|c|c|c|c|c|c|c|}
\hline & Initial & Final & Difference & & \multirow{2}{*}{\multicolumn{2}{|c|}{$\begin{array}{r}\text { Total Input } 339.7 \\
\text { Total Collected } 319.3\end{array}$}} \\
\hline Water Input & 862.0 & 522.3 & 339.7 & $100 \%$ & & \\
\hline Evap & 108.0 & 107.9 & 0.1 & \multirow[b]{6}{*}{$53.8 \%$} & \multirow{2}{*}{\multicolumn{2}{|c|}{ 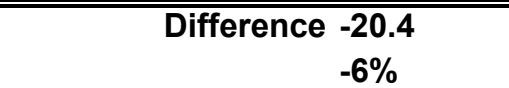 }} \\
\hline $\mathrm{G} 1-1$ & 64.9 & 240.9 & 176 & & & \\
\hline G1-2 & 56.3 & 63.0 & 6.7 & & & \\
\hline G1-3 & & & 0 & & Evaporatio & \\
\hline G1-4 & & & 0 & & Evap Pan Tare Wt, g & 66.7 \\
\hline SS-1 & & & 0 & & Evaporation, g & \\
\hline $\bar{G} 2-1$ & & & 0 & \multirow[b]{5}{*}{$0.0 \%$} & \multirow{2}{*}{\multicolumn{2}{|c|}{ \% Evaporation }} \\
\hline G2-2 & & & 0 & & & \\
\hline G2-3 & & & 0 & & & \\
\hline G2-4 & & & 0 & & \multicolumn{2}{|c|}{ Mass Flow Rate } \\
\hline SS-2 & & & 0 & & \multirow{3}{*}{$\begin{array}{l}\text { Test Duration, } \mathrm{min} \\
\text { Flow Rate, } \mathrm{g} / \mathrm{min}\end{array}$} & 10 \\
\hline G3-1 & 41.7 & 43.2 & 1.5 & & & 33.97 \\
\hline G3-2 & & & 0 & & & \\
\hline G3-3 & & & 0 & & & \\
\hline
\end{tabular}

\begin{tabular}{|c|c|c|c|c|}
\hline G3-4 & & & 0 & \\
\hline SS-3 & & & 0 & $0.4 \%$ \\
\hline G4-1 & 41.2 & 41.5 & 0.3 & \multirow[b]{5}{*}{$0.1 \%$} \\
\hline G4-2 & & & 0 & \\
\hline G4-3 & & & 0 & \\
\hline G4-4 & & & 0 & \\
\hline SS-4 & & & 0 & \\
\hline B1 & & & 0 & $0.0 \%$ \\
\hline B2 & 107.7 & 110.6 & 2.9 & $0.9 \%$ \\
\hline B3 & & & 0 & $0.0 \%$ \\
\hline B4 & 107.4 & 107.9 & 0.5 & $0.1 \%$ \\
\hline B5 & 109.3 & 113.5 & 4.2 & $1.2 \%$ \\
\hline B6 & 110.0 & 111.0 & 1 & $0.3 \%$ \\
\hline DS in-1 & & & 0 & \multirow[b]{2}{*}{$0.0 \%$} \\
\hline DS in-2 & & & 0 & \\
\hline$\overline{\text { DS out-1 }}$ & 64.8 & 167.8 & 103 & \multirow[b]{4}{*}{$37.2 \%$} \\
\hline DS out-2 & 56.2 & 79.4 & 23.2 & \\
\hline DS out-3 & & & 0 & \\
\hline$\overline{\text { DS out- } 4}$ & & & 0 & \\
\hline Other & & & 0 & $0.0 \%$ \\
\hline
\end{tabular}

\begin{tabular}{|c|c|c|c|c|}
\hline \multicolumn{5}{|c|}{ Test Chamber Environment } \\
\hline Initial Conditions & $\mathrm{RH}(\%)$ & Temperature $\left({ }^{\circ} \mathrm{C}\right)$ & & Atm. P. (mbar) \\
\hline 1-HUM/TMP-BDST & 88.8 & 26.3 & P-IN-BDST & 864 \\
\hline 2-HUM/TMP-BDST & 88.7 & 26.1 & & \\
\hline Final Conditions & $\mathrm{RH}(\%)$ & Temperature $\left({ }^{\circ} \mathrm{C}\right)$ & & Atm. P. (mbar) \\
\hline 1-HUM/TMP-BDST & 93.6 & 27.7 & P-IN-BDST & 863 \\
\hline 2-HUM/TMP-BDST & 94.6 & 27.5 & & \\
\hline
\end{tabular}


Bounding Flow Test Summary and Mass Balance-Smooth DS Surface

Test: Low flow rate, $27 \mathrm{~cm}$ right of DS Center, 16.5

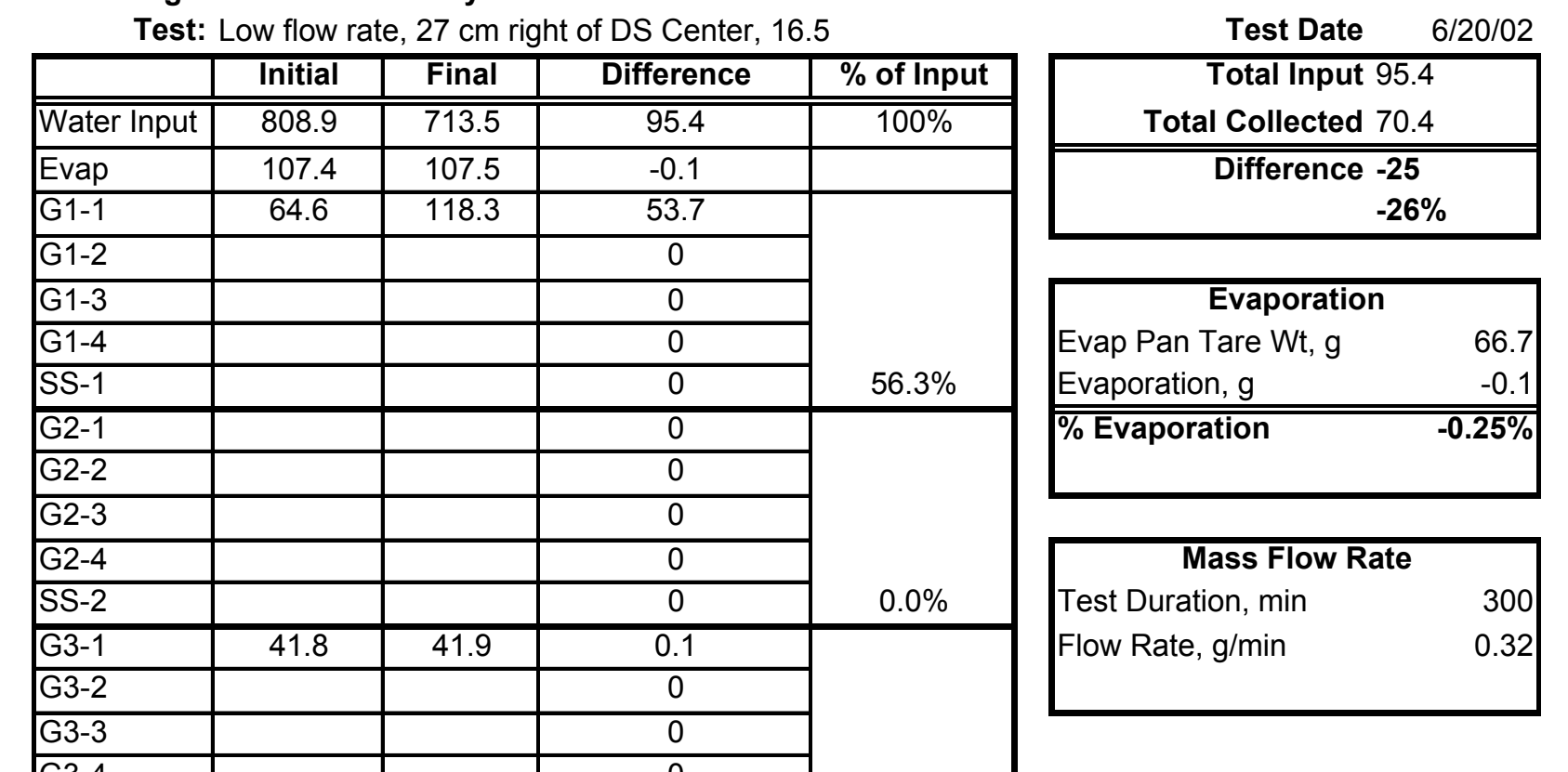

\begin{tabular}{|c|c|c|c|c|}
\hline G3-4 & & & 0 & \\
\hline SS-3 & & & 0 & $0.1 \%$ \\
\hline$\overline{\mathrm{G} 4-1}$ & 41.1 & 41.1 & 0 & \multirow[b]{5}{*}{$0.0 \%$} \\
\hline G4-2 & & & 0 & \\
\hline G4-3 & & & 0 & \\
\hline G4-4 & & & 0 & \\
\hline SS-4 & & & 0 & \\
\hline B1 & & & 0 & $0.0 \%$ \\
\hline$\overline{B 2}$ & 108.5 & 108.6 & 0.1 & $0.1 \%$ \\
\hline B3 & & & 0 & $0.0 \%$ \\
\hline B4 & & & 0 & $0.0 \%$ \\
\hline B5 & 109.3 & 110.4 & 1.1 & $1.2 \%$ \\
\hline B6 & 110.0 & 110.7 & 0.7 & $0.7 \%$ \\
\hline DS in-1 & & & 0 & \multirow[b]{2}{*}{$0.0 \%$} \\
\hline$\overline{D S}$ in-2 & & & 0 & \\
\hline DS out-1 & 63.7 & 78.4 & 14.7 & \multirow[b]{4}{*}{$15.4 \%$} \\
\hline DS out-2 & & & 0 & \\
\hline DS out-3 & & & 0 & \\
\hline DS out-4 & & & 0 & \\
\hline Other & & & 0 & $0.0 \%$ \\
\hline
\end{tabular}

\begin{tabular}{|lcccc|}
\hline \multicolumn{4}{l}{ Test Chamber Environment } & \multicolumn{3}{l|}{} \\
\hline Initial Conditions & $\mathrm{RH}(\%)$ & Temperature $\left({ }^{\circ} \mathrm{C}\right)$ & Atm. P. (mbar) \\
1-HUM/TMP-BDST & 86.6 & 26.3 & P-IN-BDST & 857 \\
2-HUM/TMP-BDST & 87.1 & 26.3 & & \\
\hline Final Conditions & $\mathrm{RH}(\%)$ & Temperature $\left({ }^{\circ} \mathrm{C}\right)$ & & Atm. P. (mbar) \\
1-HUM/TMP-BDST & 95.8 & 29.9 & P-IN-BDST & 860 \\
2-HUM/TMP-BDST & 93.4 & 29.8 & & \\
\hline
\end{tabular}


Bounding Flow Test Summary and Mass Balance-Smooth DS Surface

Test: High flow rate, $54 \mathrm{~cm}$ left of DS center, 33

\begin{tabular}{|c|c|c|c|c|}
\hline & Initial & Final & Difference & $\%$ of Input \\
\hline Water Inpu & 890.3 & 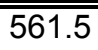 & "328.8 & $\overline{~ 100 \%}$ \\
\hline Evap & 102.0 & 101.8 & 0.2 & \\
\hline G1-1 & 65.1 & 137.9 & 72.8 & \multirow[b]{5}{*}{$22.1 \%$} \\
\hline $\mathrm{G} 1-2$ & & & 0 & \\
\hline $\mathrm{G} 1-3$ & & & 0 & \\
\hline G1-4 & & & 0 & \\
\hline SS-1 & & & 0 & \\
\hline $\bar{G} 2-1$ & 41.3 & 41.8 & 0.5 & \multirow[b]{5}{*}{$0.2 \%$} \\
\hline G2-2 & & & 0 & \\
\hline $\bar{G} 2-3$ & & & 0 & \\
\hline G2-4 & & & 0 & \\
\hline SS-2 & & & 0 & \\
\hline G3-1 & & & 0 & \multirow[b]{5}{*}{$0.0 \%$} \\
\hline G3-2 & & & 0 & \\
\hline G3-3 & & & 0 & \\
\hline G3-4 & & & 0 & \\
\hline SS-3 & & & 0 & \\
\hline$\overline{\mathrm{G} 4-1}$ & & & $\overline{0}$ & \multirow[b]{5}{*}{$0.0 \%$} \\
\hline G4-2 & & & 0 & \\
\hline G4-3 & & & 0 & \\
\hline G4-4 & & & 0 & \\
\hline SS-4 & & & 0 & \\
\hline$\overline{B 1}$ & 106.9 & 107.3 & $\overline{0.4}$ & $0.1 \%$ \\
\hline B2 & 107.5 & 108.1 & 0.6 & $0.2 \%$ \\
\hline B3 & & & 0 & $0.0 \%$ \\
\hline B4 & 107.2 & 294.1 & 186.9 & $56.8 \%$ \\
\hline B5 & & & 0 & $0.0 \%$ \\
\hline B6 & & & 0 & $0.0 \%$ \\
\hline DS in-1 & & & 0 & \multirow[b]{2}{*}{$0.0 \%$} \\
\hline DS in-2 & & & 0 & \\
\hline DS out-1 & 64.2 & $\overline{97.7}$ & 33.5 & \multirow[b]{4}{*}{$10.2 \%$} \\
\hline DS out-2 & & & 0 & \\
\hline DS out-3 & & & 0 & \\
\hline DS out-4 & & & 0 & \\
\hline Other & & & 0 & $0.0 \%$ \\
\hline
\end{tabular}

Test Date 6/20/02

\begin{tabular}{|r|}
\hline Test Date \\
\hline Total Input 328.8 \\
Total Collected 294.7 \\
\hline \hline Difference $\mathbf{- 3 4 . 1}$ \\
$-\mathbf{- 1 0 \%}$ \\
\hline
\end{tabular}

\begin{tabular}{|cc|}
\multicolumn{2}{c|}{ Evaporation } \\
Evap Pan Tare Wt, $g$ & 66.7
\end{tabular}

Evaporation, g

\% Evaporation

$0.57 \%$

\begin{tabular}{|lr|}
\hline \multicolumn{2}{|c|}{ Mass Flow Rate } \\
Test Duration, $\min$ & 10 \\
Flow Rate, g/min & 32.88 \\
\hline
\end{tabular}

\begin{tabular}{|lcccc|}
\hline \multicolumn{7}{l}{ Test Chamber Environment } & & & \\
\hline Initial Conditions & $\mathrm{RH}(\%)$ & Temperature $\left({ }^{\circ} \mathrm{C}\right)$ & & Atm. P. (mbar) \\
1-HUM/TMP-BDST & 81.8 & 27.7 & P-IN-BDST & 858 \\
2-HUM/TMP-BDST & 85.3 & 27.5 & & \\
\hline Final Conditions & $\mathrm{RH}(\%)$ & Temperature $\left({ }^{\circ} \mathrm{C}\right)$ & & Atm. P. (mbar) \\
1-HUM/TMP-BDST & 90.5 & 28.7 & P-IN-BDST & 870 \\
2-HUM/TMP-BDST & 92.9 & 28.2 & & \\
\hline
\end{tabular}

SN-M\&O-SCI-043-V2, pages 62-63 
Bounding Flow Test Summary and Mass Balance-Smooth DS Surface

Test: Low flow rate, $54 \mathrm{~cm}$ left of DS center, 33

\begin{tabular}{|c|c|c|c|c|c|c|}
\hline & Initial & Final & Difference & $\%$ of Input & \multirow{2}{*}{\multicolumn{2}{|c|}{$\begin{array}{r}\text { Total Input } 101.1 \\
\text { Total Collected } 88.4\end{array}$}} \\
\hline Water Input & 685.3 & 584.2 & 101.1 & $100 \%$ & & \\
\hline Evap & 103.7 & 104.0 & -0.3 & & \multirow{2}{*}{\multicolumn{2}{|c|}{$\begin{array}{r}\text { Difference }-12.7 \\
-13 \%\end{array}$}} \\
\hline G1-1 & 50.1 & 50.7 & 0.6 & \multirow[b]{5}{*}{$0.6 \%$} & & \\
\hline G1-2 & & & 0 & & & \\
\hline $\mathrm{G1-3}$ & & & 0 & & Evaporatio & \\
\hline G1-4 & & & 0 & & Evap Pan Tare Wt, g & 66.7 \\
\hline SS-1 & & & 0 & & Evaporation, g & -0.3 \\
\hline $\mathrm{G} 2-1$ & & & 0 & \multirow[b]{5}{*}{$0.0 \%$} & \% Evaporation & $-0.81 \%$ \\
\hline G2-2 & & & 0 & & & \\
\hline G2-3 & & & 0 & & & \\
\hline G2-4 & & & 0 & & Mass Flow R & \\
\hline SS-2 & & & 0 & & Test Duration, min & 300 \\
\hline G3-1 & & & 0 & & Flow Rate, g/min & 0.34 \\
\hline G3-2 & & & 0 & & & \\
\hline G3-3 & & & 0 & & & \\
\hline
\end{tabular}

\begin{tabular}{|c|c|c|c|c|}
\hline G3-4 & & & 0 & \\
\hline SS-3 & & & 0 & $0.0 \%$ \\
\hline G4-1 & & & 0 & \multirow[b]{5}{*}{$0.0 \%$} \\
\hline G4-2 & & & 0 & \\
\hline G4-3 & & & 0 & \\
\hline G4-4 & & & 0 & \\
\hline SS-4 & & & 0 & \\
\hline B1 & 106.6 & 107.5 & 0.9 & $0.9 \%$ \\
\hline B2 & 107.5 & 108.4 & 0.9 & $0.9 \%$ \\
\hline B3 & & & 0 & $0.0 \%$ \\
\hline B4 & 107.3 & 190.4 & 83.1 & $82.2 \%$ \\
\hline B5 & & & 0 & $0.0 \%$ \\
\hline B6 & & & 0 & $0.0 \%$ \\
\hline DS in-1 & & & 0 & \multirow[b]{2}{*}{$0.0 \%$} \\
\hline DS in-2 & & & 0 & \\
\hline DS out-1 & 58.0 & 60.9 & 2.9 & \multirow[b]{4}{*}{$2.9 \%$} \\
\hline DS out-2 & & & 0 & \\
\hline DS out-3 & & & 0 & \\
\hline DS out-4 & & & 0 & \\
\hline Other & & & 0 & $0.0 \%$ \\
\hline
\end{tabular}

\begin{tabular}{|lcccc|}
\hline \multicolumn{5}{l}{ Test Chamber Environment } \\
\hline Initial Conditions & $\mathrm{RH}(\%)$ & Temperature $\left({ }^{\circ} \mathrm{C}\right)$ & Atm. P. (mbar) \\
1-HUM/TMP-BDST & 81.2 & 25.3 & P-IN-BDST & 865 \\
2-HUM/TMP-BDST & 83.1 & 25.4 & & \\
\hline Final Conditions & $\mathrm{RH}(\%)$ & Temperature $\left({ }^{\circ} \mathrm{C}\right)$ & Atm. P. (mbar) \\
1-HUM/TMP-BDST & 107.4 & 27.3 & P-IN-BDST & 874 \\
2-HUM/TMP-BDST & 95.9 & 27.2 & & \\
\hline
\end{tabular}


Bounding Flow Test Summary and Mass Balance-Smooth DS Surface

Test: High flow rate, $27 \mathrm{~cm}$ left of DS center, 33

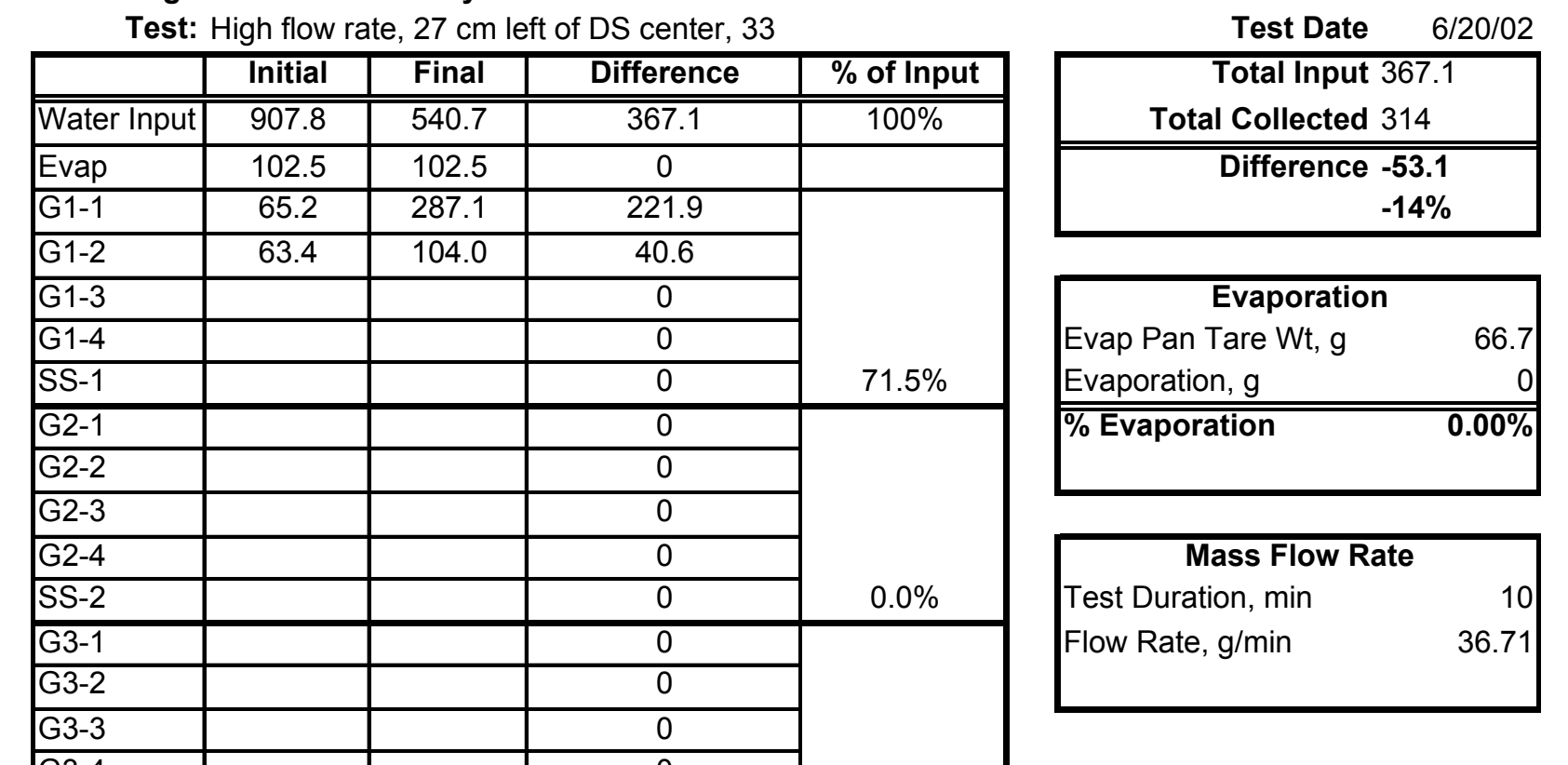

\begin{tabular}{|c|c|c|c|c|}
\hline & & & & \multirow[b]{3}{*}{$0.0 \%$} \\
\hline G3-4 & & & 0 & \\
\hline SS-3 & & & 0 & \\
\hline G4-1 & & & 0 & \multirow[b]{5}{*}{$0.0 \%$} \\
\hline$\overline{\mathrm{G}} 4-2$ & & & 0 & \\
\hline G4-3 & & & 0 & \\
\hline G4-4 & & & 0 & \\
\hline$\overline{S S-4}$ & & & 0 & \\
\hline $\mathrm{B} 1$ & & & 0 & $0.0 \%$ \\
\hline B2 & 107.7 & 110.6 & 2.9 & $0.8 \%$ \\
\hline B3 & & & 0 & $0.0 \%$ \\
\hline B4 & 107.1 & 108.1 & 1 & $0.3 \%$ \\
\hline B5 & 109.8 & 110.9 & 1.1 & $0.3 \%$ \\
\hline B6 & & & 0 & $0.0 \%$ \\
\hline DS in-1 & & & 0 & \multirow[b]{2}{*}{$0.0 \%$} \\
\hline DS in-2 & & & 0 & \\
\hline DS out-1 & 72.2 & 118.7 & 46.5 & \multirow[b]{4}{*}{$12.7 \%$} \\
\hline DS out-2 & & & 0 & \\
\hline DS out-3 & & & 0 & \\
\hline DS out-4 & & & 0 & \\
\hline Other & & & 0 & $0.0 \%$ \\
\hline
\end{tabular}

$\begin{array}{lll}\text { Total Collected } & 314 & 85.5 \%\end{array}$

\begin{tabular}{|lcccc|}
\hline \multicolumn{7}{l}{ Test Chamber Environment } & & & \\
\hline Initial Conditions & $\mathrm{RH}(\%)$ & Temperature $\left({ }^{\circ} \mathrm{C}\right)$ & & Atm. P. (mbar) \\
1-HUM/TMP-BDST & 87.3 & 28.2 & P-IN-BDST & 857 \\
2-HUM/TMP-BDST & 89.7 & 27.6 & & \\
\hline Final Conditions & $\mathrm{RH}(\%)$ & Temperature $\left({ }^{\circ} \mathrm{C}\right)$ & & Atm. P. (mbar) \\
1-HUM/TMP-BDST & 91.9 & 29 & P-IN-BDST & 867 \\
2-HUM/TMP-BDST & 93.9 & 28.6 & & \\
\hline
\end{tabular}


Bounding Flow Test Summary and Mass Balance-Smooth DS Surface

Test: Low flow rate, $27 \mathrm{~cm}$ left of DS center, 33

\begin{tabular}{|c|c|c|c|c|c|c|}
\hline \multicolumn{5}{|l|}{ Test: } & \multicolumn{2}{|l|}{ Test Date } \\
\hline & Initial & Final & Difference & $\%$ of Input & \multicolumn{2}{|c|}{ Total Input 96.3} \\
\hline Water Input & -1.9 & $\overline{-98.2}$ & 96.3 & $100 \%$ & \multicolumn{2}{|c|}{ Total Collected 86.3} \\
\hline Evap & 103.4 & 102.8 & 0.6 & & \multirow{2}{*}{\multicolumn{2}{|c|}{$\begin{array}{r}\text { Difference }-10 \\
-10 \%\end{array}$}} \\
\hline G1-1 & 64.6 & 142.8 & 78.2 & \multirow[b]{5}{*}{$81.2 \%$} & & \\
\hline G1-2 & & & 0 & & \\
\hline G1-3 & & & 0 & & \multicolumn{2}{|c|}{ Evaporation } \\
\hline G1-4 & & & 0 & & \multirow{2}{*}{$\begin{array}{l}\text { Evap Pan Tare Wt, g } \\
\text { Evaporation, g }\end{array}$} & \multirow{2}{*}{$\begin{array}{r}66.7 \\
0.6\end{array}$} \\
\hline SS-1 & & & 0 & & & \\
\hline$\overline{\mathrm{G} 2-1}$ & & & 0 & \multirow[b]{5}{*}{$0.0 \%$} & \multirow{2}{*}{\multicolumn{2}{|c|}{ \% Evaporation }} \\
\hline G2-2 & & & 0 & & & \\
\hline G2-3 & & & 0 & & \multirow{2}{*}{\multicolumn{2}{|c|}{ Mass Flow Rate }} \\
\hline $\bar{G} 2-4$ & & & 0 & & & \\
\hline SS-2 & & & 0 & & \multirow{3}{*}{$\begin{array}{l}\text { Test Duration, min } \\
\text { Flow Rate, g/min }\end{array}$} & \multirow{3}{*}{$\begin{array}{r}304.95 \\
0.32\end{array}$} \\
\hline$\overline{G 3-1}$ & & & $\overline{0}$ & & & \\
\hline G3-2 & & & 0 & & & \\
\hline G3-3 & & & 0 & & & \\
\hline
\end{tabular}

\begin{tabular}{|c|c|c|c|c|}
\hline G3-4 & & & 0 & \multirow[b]{2}{*}{$0.0 \%$} \\
\hline SS-3 & & & 0 & \\
\hline$\overline{\mathrm{G} 4-1}$ & & & $\overline{0}$ & \multirow[b]{5}{*}{$0.0 \%$} \\
\hline G4-2 & & & 0 & \\
\hline G4-3 & & & 0 & \\
\hline G4-4 & & & 0 & \\
\hline SS-4 & & & 0 & \\
\hline B1 & & & 0 & $0.0 \%$ \\
\hline B2 & 106.7 & 107.6 & 0.9 & $0.9 \%$ \\
\hline B3 & & & 0 & $0.0 \%$ \\
\hline B4 & 109.8 & 111.0 & 1.2 & $1.2 \%$ \\
\hline B5 & 107.2 & 107.9 & 0.7 & $0.7 \%$ \\
\hline $\mathrm{B} 6$ & 109.0 & 109.8 & 0.8 & $0.8 \%$ \\
\hline DS in-1 & & & 0 & \multirow[b]{2}{*}{$0.0 \%$} \\
\hline DS in-2 & & & $\overline{0}$ & \\
\hline DS out-1 & 56.9 & 61.4 & 4.5 & \multirow[b]{4}{*}{$4.7 \%$} \\
\hline DS out- 2 & & & 0 & \\
\hline DS out- 3 & & & 0 & \\
\hline$\overline{D S}$ out- 4 & & & 0 & \\
\hline Other & & & 0 & $0.0 \%$ \\
\hline
\end{tabular}

Test Chamber Environment

\begin{tabular}{|lcccc|}
\hline Initial Conditions & $\mathrm{RH}(\%)$ & Temperature $\left({ }^{\circ} \mathrm{C}\right)$ & Atm. P. (mbar) \\
1-HUM/TMP-BDST & 83.5 & 23.9 & P-IN-BDST & 863 \\
2-HUM/TMP-BDST & 83.9 & 23.9 & & \\
\hline Final Conditions & $\mathrm{RH}(\%)$ & Temperature $\left({ }^{\circ} \mathrm{C}\right)$ & & Atm. P. (mbar) \\
1-HUM/TMP-BDST & 96.9 & 28.4 & P-IN-BDST & 861 \\
2-HUM/TMP-BDST & 95 & 27.8 & & \\
\hline
\end{tabular}


Bounding Flow Test Summary and Mass Balance-Smooth DS Surface

Test: High flow rate, $27 \mathrm{~cm}$ right of DS center, 33

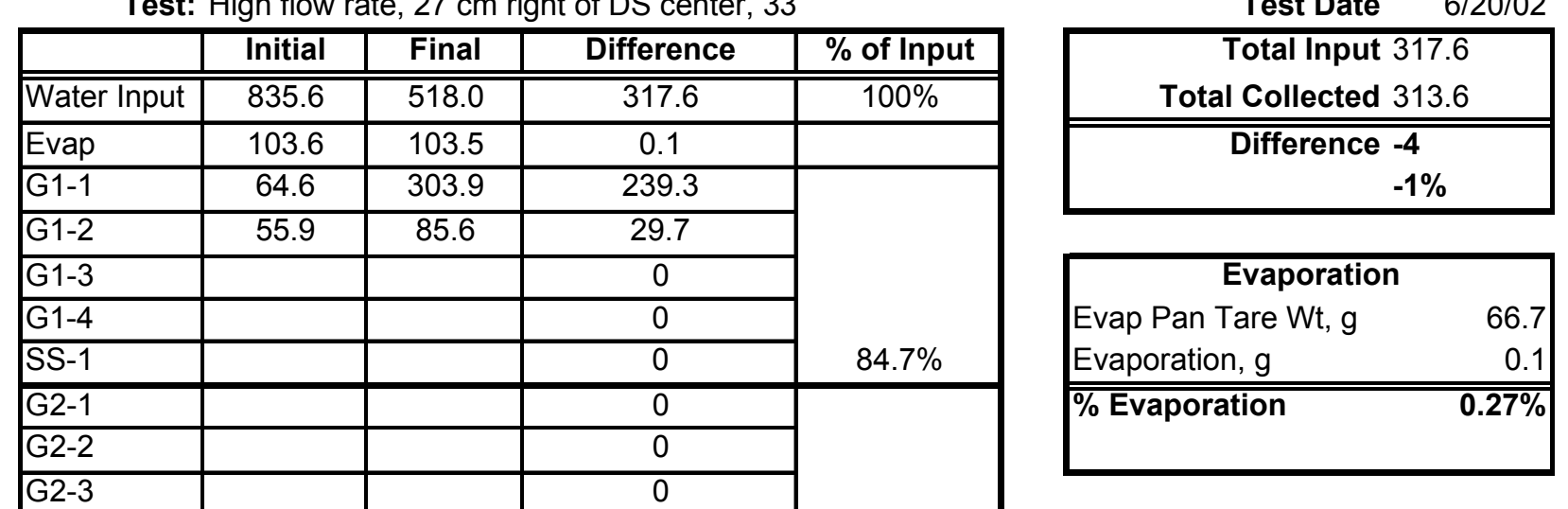

\begin{tabular}{|c|c|c|c|c|c|}
\hline & & & & \multirow[b]{3}{*}{$0.0 \%$} & \multirow{2}{*}{ Mass Flow Rate } \\
\hline G2-4 & & & 0 & & \\
\hline SS-2 & & & 0 & & Test Duration, min \\
\hline$\overline{\mathrm{G} 3-1}$ & 41.9 & 41.9 & $\overline{0}$ & & Flow Rate, g/min \\
\hline $\bar{G} 3-2$ & & & 0 & & \\
\hline G3-3 & & & 0 & & \\
\hline
\end{tabular}

\begin{tabular}{|c|c|c|c|c|}
\hline G3-4 & & & 0 & \\
\hline SS-3 & & & 0 & $0.0 \%$ \\
\hline G4-1 & & & 0 & \multirow[b]{5}{*}{$0.0 \%$} \\
\hline G4-2 & & & $\overline{0}$ & \\
\hline G4-3 & & & 0 & \\
\hline G4-4 & & & 0 & \\
\hline SS-4 & & & 0 & \\
\hline $\mathrm{B} 1$ & & & $\overline{0}$ & $0.0 \%$ \\
\hline B2 & 107.6 & 108.4 & 0.8 & $0.3 \%$ \\
\hline B3 & & & 0 & $0.0 \%$ \\
\hline B4 & 107.3 & 107.9 & 0.6 & $0.2 \%$ \\
\hline B5 & 109.9 & 113.5 & 3.6 & $1.1 \%$ \\
\hline B6 & & & 0 & $0.0 \%$ \\
\hline$\overline{D S}$ in-1 & & & 0 & \multirow[b]{2}{*}{$0.0 \%$} \\
\hline$\overline{D S}$ in-2 & & & 0 & \\
\hline DS out-1 & 72.0 & 111.6 & 39.6 & \multirow[b]{4}{*}{$12.5 \%$} \\
\hline DS out- 2 & & & 0 & \\
\hline DS out-3 & & & 0 & \\
\hline \begin{tabular}{|l|} 
DS out- 4 \\
\end{tabular} & & & 0 & \\
\hline Other & & & 0 & $0.0 \%$ \\
\hline
\end{tabular}

\begin{tabular}{|lcccc|}
\hline \multicolumn{7}{l}{ Test Chamber Environment } & & & \\
\hline Initial Conditions & $\mathrm{RH}(\%)$ & Temperature $\left({ }^{\circ} \mathrm{C}\right)$ & & Atm. P. (mbar) \\
1-HUM/TMP-BDST & 88.8 & 28.4 & P-IN-BDST & 862 \\
2-HUM/TMP-BDST & 85.1 & 28.3 & & \\
\hline Final Conditions & $\mathrm{RH}(\%)$ & Temperature $\left({ }^{\circ} \mathrm{C}\right)$ & & Atm. P. (mbar) \\
1-HUM/TMP-BDST & 92.5 & 29.2 & P-IN-BDST & 854 \\
2-HUM/TMP-BDST & 93 & 29 & & \\
\hline
\end{tabular}


Bounding Flow Test Summary and Mass Balance-Smooth DS Surface

Test: Low flow rate, $27 \mathrm{~cm}$ right of DS center, 33

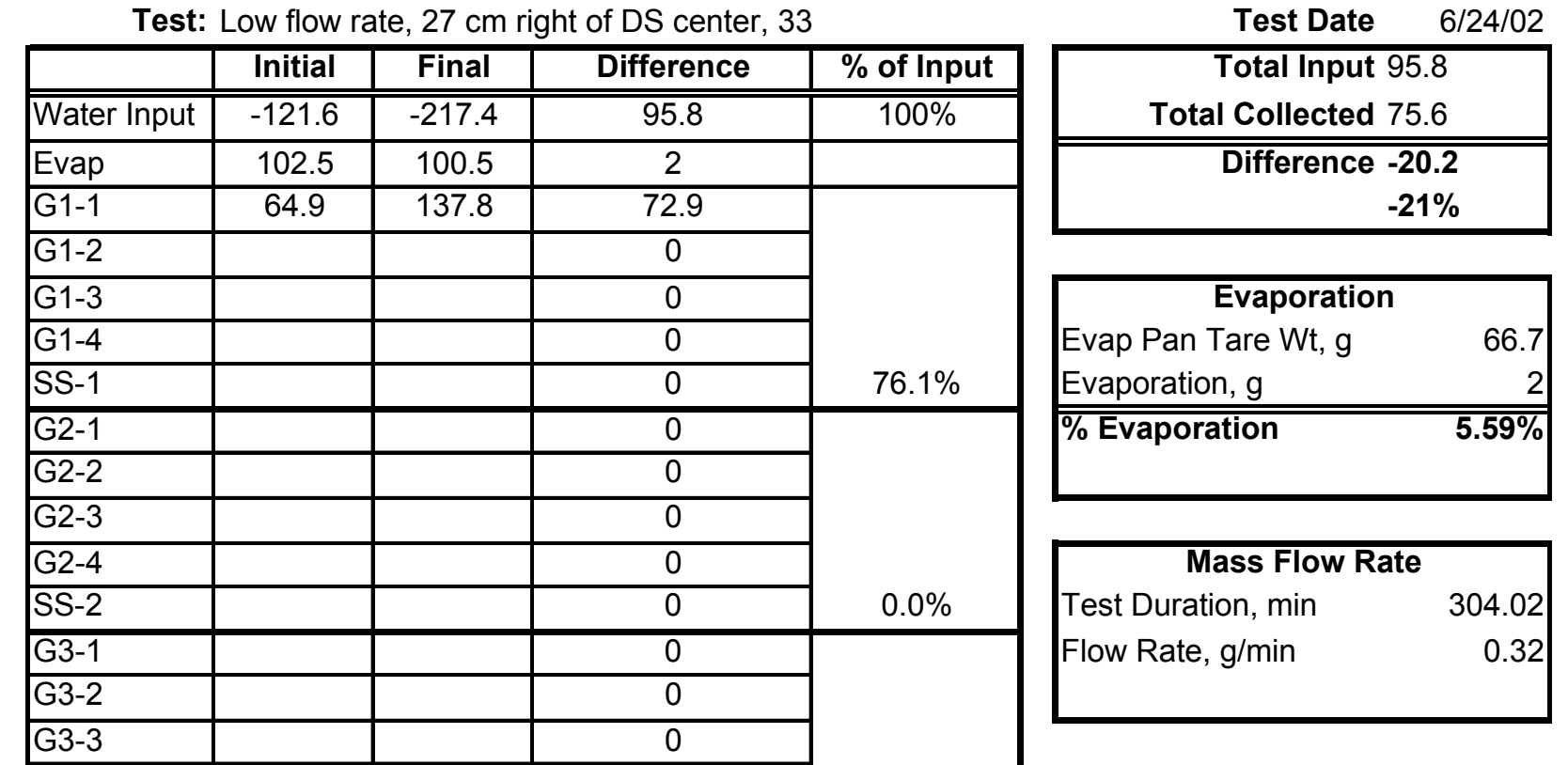

G3-4

SS-3

0

G4-1

G4-2

G4-3

G4-4

SS-4

$\mathrm{B} 1$

$\mathrm{B} 2$

B3

B4

B5

B6

\begin{tabular}{|c|c|c|c|c|}
\hline DS in-1 & & & 0 & \multirow[b]{2}{*}{$0.0 \%$} \\
\hline DS in-2 & & & 0 & \\
\hline DS out-1 & 49.6 & 51.9 & 2.3 & \multirow[b]{4}{*}{$2.4 \%$} \\
\hline DS out-2 & & & 0 & \\
\hline DS out-3 & & & 0 & \\
\hline DS out-4 & & & 0 & \\
\hline Other & & & 0 & $0.0 \%$ \\
\hline
\end{tabular}

Test Chamber Environment

\begin{tabular}{|lcccc|}
\hline Initial Conditions & $\mathrm{RH}(\%)$ & Temperature $\left({ }^{\circ} \mathrm{C}\right)$ & & Atm. P. (mbar) \\
1-HUM/TMP-BDST & 93.9 & 28.6 & P-IN-BDST & 856 \\
2-HUM/TMP-BDST & 92.6 & 28.3 & & \\
\hline Final Conditions & $\mathrm{RH}(\%)$ & Temperature $\left({ }^{\circ} \mathrm{C}\right)$ & & Atm. P. (mbar) \\
1-HUM/TMP-BDST & 96.9 & 29.6 & P-IN-BDST & 892 \\
2-HUM/TMP-BDST & 93.5 & 29.3 & & \\
\hline
\end{tabular}


Single Patch q(splash) Test Summary and Mass Balance - Rough Surface

Test: Patch 4, $27 \mathrm{~cm}$ right of patch center, 33

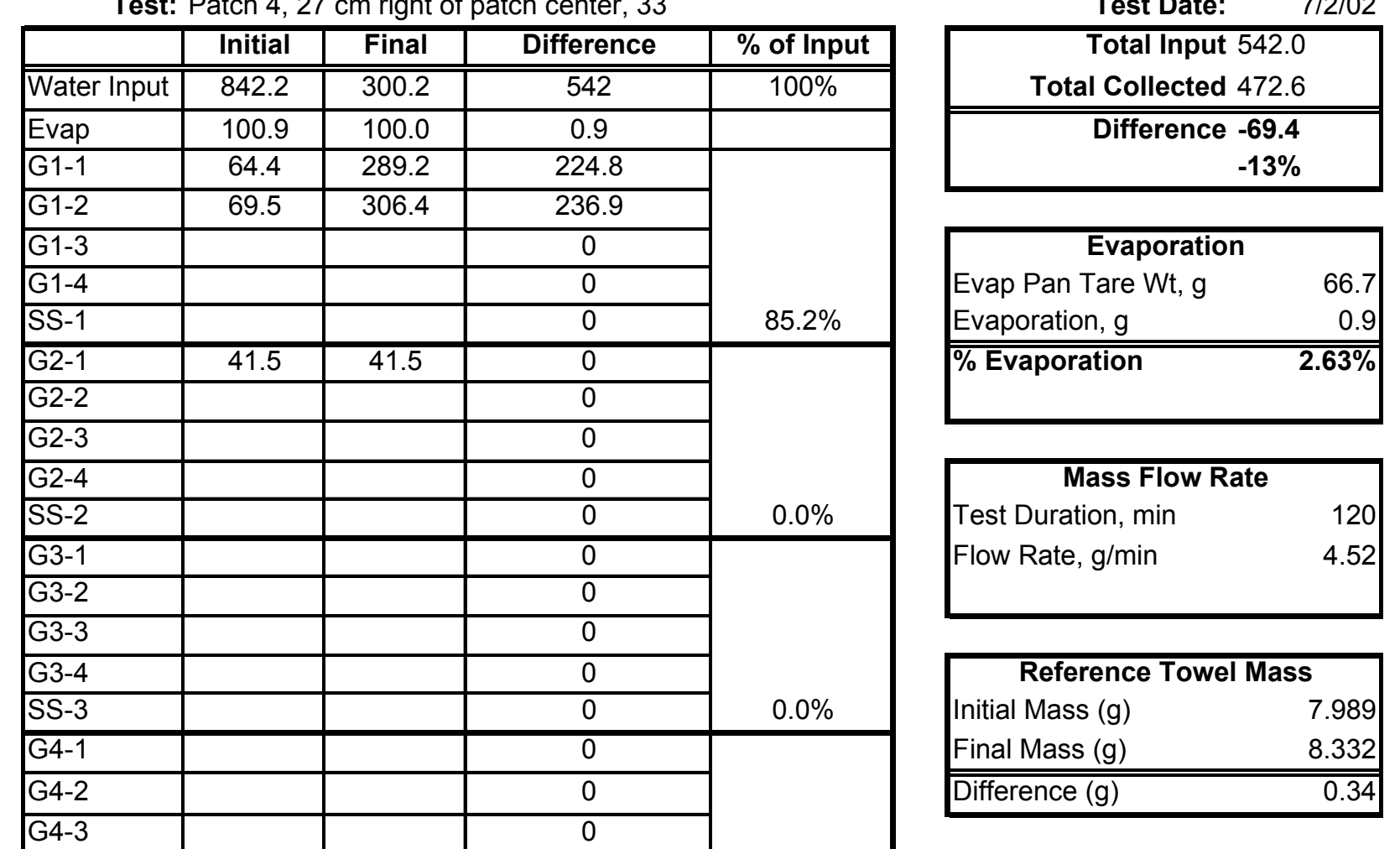

\begin{tabular}{|c|c|c|c|c|}
\hline & & & & \multirow[b]{3}{*}{$0.0 \%$} \\
\hline G4-4 & & & 0 & \\
\hline SS-4 & & & 0 & \\
\hline B1 & & & 0 & $0.0 \%$ \\
\hline B2 & & & 0 & $0.0 \%$ \\
\hline B3 & & & 0 & $0.0 \%$ \\
\hline B4 & 107.5 & 107.8 & 0.3 & $0.1 \%$ \\
\hline B5 & & & 0 & $0.0 \%$ \\
\hline B6 & & & 0 & $0.0 \%$ \\
\hline DS in-1 & & & 0 & \multirow[b]{2}{*}{$0.0 \%$} \\
\hline DS in-2 & & & 0 & \\
\hline DS out-1 & 64.4 & 75.0 & 10.6 & \multirow[b]{4}{*}{$2.0 \%$} \\
\hline DS out-2 & & & 0 & \\
\hline DS out-3 & & & 0 & \\
\hline DS out-4 & & & 0 & \\
\hline Other & & & 0 & $0.0 \%$ \\
\hline
\end{tabular}

\begin{tabular}{|lcccc|}
\hline \multicolumn{7}{|l|}{ Test Chamber Environment } & & & \\
\hline Initial Conditions & $\mathrm{RH}(\%)$ & Temperature $\left({ }^{\circ} \mathrm{C}\right)$ & Atm. P. (mbar) \\
1-HUM/TMP-BDST & 85.3 & 27.4 & P-IN-BDST & 869 \\
2-HUM/TMP-BDST & 85.6 & 27.3 & & \\
\hline Final Conditions & $\mathrm{RH}(\%)$ & Temperature $\left({ }^{\circ} \mathrm{C}\right)$ & & Atm. P. (mbar) \\
1-HUM/TMP-BDST & 94.2 & 29.2 & P-IN-BDST & 866 \\
2-HUM/TMP-BDST & 92.4 & 29.1 & & \\
\hline
\end{tabular}

SN-M\&O-SCl-043-V2, pages 103-104 
Single Patch q(splash) Test Summary and Mass Balance - Rough Surface

Test: Patch 5, $20 \mathrm{~cm}$ left of patch center, 33

\begin{tabular}{|c|c|c|c|c|}
\hline & Initial & Final & Difference & $\%$ of Input \\
\hline Water Input & 880.2 & $\overline{349.2}$ & 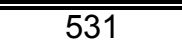 & $\overline{~ 100 \%}$ \\
\hline$\overline{\text { Evap }}$ & 116.9 & 116.1 & 0.8 & \\
\hline G1-1 & 64.8 & 302.5 & 237.7 & \multirow[b]{5}{*}{$83.6 \%$} \\
\hline G1-2 & 70.6 & 276.7 & 206.1 & \\
\hline G1-3 & & & 0 & \\
\hline $\mathrm{G} 1-4$ & & & 0 & \\
\hline SS-1 & & & 0 & \\
\hline$\overline{G 2-1}$ & & & 0 & \multirow[b]{5}{*}{$0.0 \%$} \\
\hline G2-2 & & & $\overline{0}$ & \\
\hline G2-3 & & & 0 & \\
\hline G2-4 & & & 0 & \\
\hline SS-2 & & & 0 & \\
\hline$\overline{\mathrm{G} 3-1}$ & & & $\overline{0}$ & \multirow[b]{5}{*}{$0.0 \%$} \\
\hline G3-2 & & & 0 & \\
\hline$\overline{\text { G3-3 }}$ & & & 0 & \\
\hline G3-4 & & & 0 & \\
\hline SS-3 & & & 0 & \\
\hline$\overline{\mathrm{G} 4-1}$ & 41.5 & 41.5 & 0 & \multirow[b]{5}{*}{$0.0 \%$} \\
\hline G4-2 & & & 0 & \\
\hline G4-3 & & & 0 & \\
\hline $\mathrm{G} 4-4$ & & & 0 & \\
\hline SS-4 & & & 0 & \\
\hline B1 & & & 0 & $0.0 \%$ \\
\hline $\bar{B} 2$ & & & 0 & $0.0 \%$ \\
\hline$\overline{B 3}$ & 107.1 & 107.8 & 0.7 & $0.1 \%$ \\
\hline B4 & & & 0 & $0.0 \%$ \\
\hline B5 & 109.6 & 111.3 & 1.7 & $0.3 \%$ \\
\hline B6 & & & 0 & $0.0 \%$ \\
\hline DS in-1 & & & 0 & \multirow[b]{2}{*}{$0.0 \%$} \\
\hline$\overline{\mathrm{DS}}$ in-2 & & & 0 & \\
\hline DS out-1 & 65.1 & 79.7 & 14.6 & \multirow[b]{4}{*}{$2.7 \%$} \\
\hline DS out-2 & & & 0 & \\
\hline DS out-3 & & & 0 & \\
\hline DS out-4 & & & 0 & \\
\hline Other & & & 0 & $0.0 \%$ \\
\hline
\end{tabular}

Test Date: $\quad 7 / 2 / 02$

\begin{tabular}{|r|}
\hline Total Input 531.0 \\
Total Collected 460.8 \\
\hline \hline Difference $\mathbf{- 7 0 . 2}$ \\
$-\mathbf{- 1 3 \%}$ \\
\hline
\end{tabular}

\begin{tabular}{|lr|}
\hline \multicolumn{2}{|c|}{ Evaporation } \\
Evap Pan Tare Wt, g & 66.7 \\
Evaporation, g & 0.8 \\
\hline \% Evaporation & $\mathbf{1 . 5 9 \%}$ \\
\hline
\end{tabular}

\begin{tabular}{|lr|}
\hline \multicolumn{2}{|c|}{ Mass Flow Rate } \\
Test Duration, min & 120.02 \\
Flow Rate, g/min & 4.42 \\
\hline
\end{tabular}

\begin{tabular}{|lr|}
\hline \multicolumn{2}{|c|}{ Reference Towel Mass } \\
Initial Mass $(\mathrm{g})$ & 8.267 \\
Final Mass $(\mathrm{g})$ & 8.498 \\
\hline \hline Difference $(\mathrm{g})$ & 0.23 \\
\hline
\end{tabular}

\begin{tabular}{|c|c|c|c|c|}
\hline \multicolumn{5}{|c|}{ Test Chamber Environment } \\
\hline Initial Conditions & $\mathrm{RH}(\%)$ & Temperature $\left({ }^{\circ} \mathrm{C}\right)$ & & Atm. P. (mbar) \\
\hline 1-HUM/TMP-BDST & 90.3 & 27.5 & P-IN-BDST & 860 \\
\hline 2-HUM/TMP-BDST & 88.8 & 27.3 & & \\
\hline Final Conditions & $\mathrm{RH}(\%)$ & Temperature $\left({ }^{\circ} \mathrm{C}\right)$ & & Atm. P. (mbar) \\
\hline 1-HUM/TMP-BDST & 93.5 & 29.1 & P-IN-BDST & 870 \\
\hline 2-HUM/TMP-BDST & 93.7 & 28.7 & & \\
\hline
\end{tabular}


Single Patch q(splash) Test Summary and Mass Balance - Rough Surface

Test: Patch 5, $20 \mathrm{~cm}$ left of patch center, 16.5

\begin{tabular}{|c|c|c|c|c|c|c|}
\hline \multicolumn{5}{|c|}{ Test: } & \multicolumn{2}{|l|}{ Test Date: } \\
\hline & Initial & Final & Difference & $\%$ of Input & \multirow{2}{*}{\multicolumn{2}{|c|}{$\begin{array}{r}\text { Total Input } 536.0 \\
\text { Total Collected } 456.5\end{array}$}} \\
\hline Water Input & 815.1 & 279.1 & $\overline{c 536}$ & $100 \%$ & & \\
\hline Evap & 98.1 & 97.6 & 0.5 & & \multirow{2}{*}{\multicolumn{2}{|c|}{$\begin{array}{r}\text { Difference }-79.5 \\
-15 \%\end{array}$}} \\
\hline G1-1 & 66.4 & 286.1 & 219.7 & \multirow[b]{5}{*}{$73.9 \%$} & & \\
\hline$\overline{G 1-2}$ & 64.6 & 241.1 & 176.5 & & \\
\hline$\overline{G 1-3}$ & & & 0 & & \multicolumn{2}{|c|}{ Evaporation } \\
\hline G1-4 & & & 0 & & \multirow{2}{*}{$\begin{array}{l}\text { Evap Pan Tare Wt, g } \\
\text { Evaporation, g }\end{array}$} & 66.7 \\
\hline SS-1 & & & 0 & & & \\
\hline $\bar{G} 2-1$ & & & 0 & \multirow[b]{5}{*}{$0.0 \%$} & \multirow{2}{*}{\multicolumn{2}{|c|}{ \% Evaporation }} \\
\hline G2-2 & & & 0 & & & \\
\hline G2-3 & & & 0 & & & \\
\hline G2-4 & & & 0 & & \multicolumn{2}{|c|}{ Mass Flow Rate } \\
\hline SS-2 & & & 0 & & \multirow{3}{*}{$\begin{array}{l}\text { Test Duration, min } \\
\text { Flow Rate, g/min }\end{array}$} & 113.42 \\
\hline G3-1 & 49.8 & 50.7 & 0.9 & \multirow[b]{5}{*}{$0.2 \%$} & & 4.73 \\
\hline G3-2 & & & 0 & & & \\
\hline G3-3 & & & 0 & & & \\
\hline G3-4 & & & 0 & & \multicolumn{2}{|c|}{ Reference Towel Mass } \\
\hline SS-3 & & & 0 & & \multirow{2}{*}{$\begin{array}{l}\text { Initial Mass }(\mathrm{g}) \\
\text { Final Mass }(\mathrm{g}) \\
\end{array}$} & 7.758 \\
\hline$\overline{\mathrm{G} 4-1}$ & & & 0 & & & 8.425 \\
\hline G4-2 & & & 0 & & Difference (g) & 0.67 \\
\hline G4-3 & & & 0 & & & \\
\hline
\end{tabular}

\begin{tabular}{|c|c|c|c|c|}
\hline & & & & \multirow[b]{3}{*}{$0.0 \%$} \\
\hline G4-4 & & & 0 & \\
\hline SS-4 & & & 0 & \\
\hline B1 & & & 0 & $0.0 \%$ \\
\hline B2 & & & 0 & $0.0 \%$ \\
\hline B3 & & & 0 & $0.0 \%$ \\
\hline B4 & & & 0 & $0.0 \%$ \\
\hline B5 & 109.5 & 118.5 & 9 & $1.7 \%$ \\
\hline B6 & 110.0 & 110.7 & 0.7 & $0.1 \%$ \\
\hline DS in-1 & & & 0 & \multirow[b]{2}{*}{$0.0 \%$} \\
\hline$\overline{D S}$ in-2 & & & 0 & \\
\hline DS out- 1 & 73.4 & 123.1 & 49.7 & \multirow[b]{4}{*}{$9.3 \%$} \\
\hline DS out-2 & & & 0 & \\
\hline DS out-3 & & & 0 & \\
\hline DS out- 4 & & & 0 & \\
\hline Other & & & 0 & $0.0 \%$ \\
\hline
\end{tabular}

\begin{tabular}{|lcccc|}
\hline \multicolumn{7}{l}{ Test Chamber Environment } & & & \\
\hline Initial Conditions & $\mathrm{RH}(\%)$ & Temperature $\left({ }^{\circ} \mathrm{C}\right)$ & & Atm. P. (mbar) \\
1-HUM/TMP-BDST & 84.5 & 27.9 & P-IN-BDST & 850 \\
2-HUM/TMP-BDST & 88.8 & 27.3 & & \\
\hline Final Conditions & $\mathrm{RH}(\%)$ & Temperature $\left({ }^{\circ} \mathrm{C}\right)$ & & Atm. P. (mbar) \\
1-HUM/TMP-BDST & 92.9 & 30.5 & P-IN-BDST & 873 \\
2-HUM/TMP-BDST & 93.4 & 30 & & \\
\hline
\end{tabular}


Single Patch q(splash) Test Summary and Mass Balance - Rough Surface

Test: Patch 6, $20 \mathrm{~cm}$ left of patch center, Crown

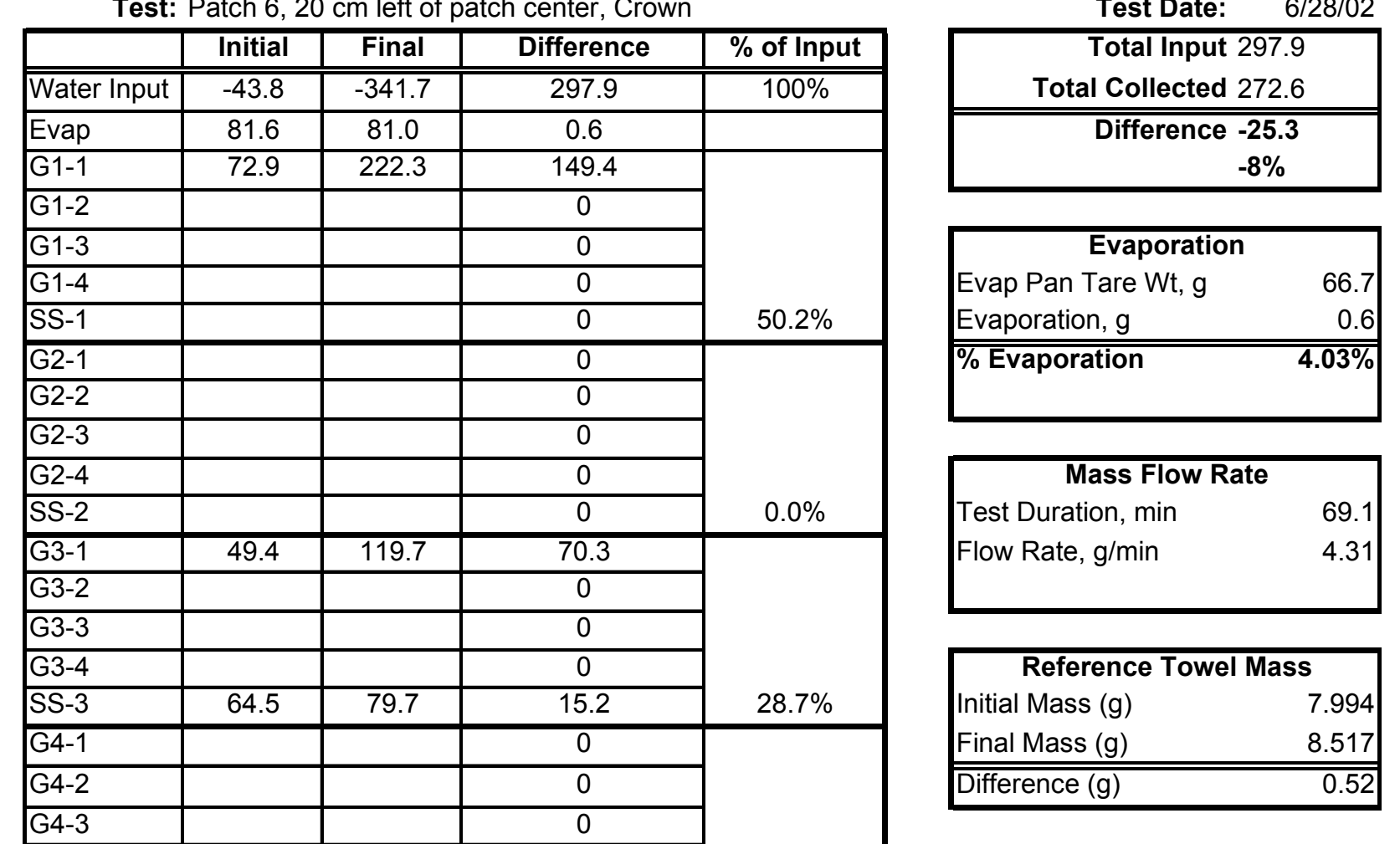

\begin{tabular}{|c|c|c|c|c|}
\hline (3-5 & & & U & \multirow[b]{3}{*}{$0.0 \%$} \\
\hline G4-4 & & & 0 & \\
\hline SS-4 & & & 0 & \\
\hline B1 & & & 0 & $0.0 \%$ \\
\hline B2 & & & 0 & $0.0 \%$ \\
\hline B3 & & & 0 & $0.0 \%$ \\
\hline B4 & 107.7 & 107.9 & 0.2 & $0.1 \%$ \\
\hline B5 & 109.5 & 110.0 & 0.5 & $0.2 \%$ \\
\hline B6 & 110.2 & 114.4 & 4.2 & $1.4 \%$ \\
\hline DS in-1 & & & 0 & \multirow[b]{2}{*}{$0.0 \%$} \\
\hline DS in-2 & & & 0 & \\
\hline DS out-1 & 64.9 & 97.7 & 32.8 & \multirow[b]{4}{*}{$11.0 \%$} \\
\hline DS out- 2 & & & 0 & \\
\hline DS out-3 & & & 0 & \\
\hline DS out-4 & & & 0 & \\
\hline Other & & & 0 & $0.0 \%$ \\
\hline
\end{tabular}

\begin{tabular}{|lcccc|}
\hline \multicolumn{5}{l}{ Test Chamber Environment } \\
\hline Initial Conditions & $\mathrm{RH}(\%)$ & Temperature $\left({ }^{\circ} \mathrm{C}\right)$ & Atm. P. (mbar) \\
1-HUM/TMP-BDST & 91 & 25.3 & P-IN-BDST & 864 \\
2-HUM/TMP-BDST & 92.7 & 25 & & \\
\hline Final Conditions & $\mathrm{RH}(\%)$ & Temperature $\left({ }^{\circ} \mathrm{C}\right)$ & Atm. P. (mbar) \\
1-HUM/TMP-BDST & 93.6 & 27.8 & P-IN-BDST & 853 \\
2-HUM/TMP-BDST & 94.3 & 27.3 & & \\
\hline
\end{tabular}


Single Patch q(splash) Test Summary and Mass Balance - Rough Surface

Test: Patch 6, Patch center, $15 \mathrm{~cm}$ below crown

\begin{tabular}{|c|c|c|c|c|c|c|}
\hline & Initial & Final & Difference & $\%$ of Input & \multirow{2}{*}{\multicolumn{2}{|c|}{ Total Collected 245.0}} \\
\hline Water Input & 6666.8 & 368.0 & 298.8 & $100 \%$ & & \\
\hline Evap & 100.9 & 100.5 & 0.4 & & \multirow{2}{*}{\multicolumn{2}{|c|}{$\begin{array}{r}\text { Difference }-53.8 \\
-18 \%\end{array}$}} \\
\hline G1-1 & 74.1 & 255.8 & 181.7 & \multirow[b]{5}{*}{$60.8 \%$} & & \\
\hline G1-2 & & & 0 & & & \\
\hline G1-3 & & & 0 & & Evaporatio & \\
\hline G1-4 & & & 0 & & Evap Pan Tare Wt, g & 66.7 \\
\hline SS-1 & & & 0 & & Evaporation, g & \\
\hline G2-1 & & & 0 & & \% Evaporation & $1.17 \%$ \\
\hline G2-2 & & & $\overline{0}$ & & & \\
\hline G2-3 & & & 0 & & & \\
\hline G2-4 & & & $\overline{0}$ & & Mass Flow R & \\
\hline SS-2 & & & 0 & $0.0 \%$ & Test Duration, min & 60 \\
\hline G3-1 & 58.1 & 79.1 & 21 & & Flow Rate, $\mathrm{g} / \mathrm{min}$ & 4.98 \\
\hline G3-2 & & & 0 & & & \\
\hline G3-3 & & & 0 & & & \\
\hline G3-4 & & & $\overline{0}$ & & Reference Towe & \\
\hline SS-3 & & & 0 & $7.0 \%$ & Initial Mass $(\mathrm{g})$ & 8.323 \\
\hline G4-1 & & & 0 & & Final Mass (g) & 8.426 \\
\hline G4-2 & & & 0 & & Difference $(\mathrm{g})$ & 0.10 \\
\hline G4-3 & & & 0 & & & \\
\hline
\end{tabular}

\begin{tabular}{|c|c|c|c|c|}
\hline G4-3 & & & 0 & \multirow[b]{3}{*}{$0.0 \%$} \\
\hline G4-4 & & & 0 & \\
\hline SS-4 & & & 0 & \\
\hline$\overline{B 1}$ & & & $\overline{0}$ & $0.0 \%$ \\
\hline B2 & & & 0 & $0.0 \%$ \\
\hline B3 & & & 0 & $0.0 \%$ \\
\hline $\bar{B} 4$ & 107.6 & 107.9 & 0.3 & $0.1 \%$ \\
\hline B5 & 109.5 & 110.0 & 0.5 & $0.2 \%$ \\
\hline B6 & 110.0 & 111.0 & 1 & $0.3 \%$ \\
\hline DS in-1 & & & 0 & \multirow[b]{2}{*}{$0.0 \%$} \\
\hline DS in-2 & & & 0 & \\
\hline$\overline{\text { DS out- } 1}$ & 57.4 & 91.5 & 34.1 & \multirow[b]{4}{*}{$13.6 \%$} \\
\hline DS out-2 & 8.1 & 14.5 & 6.4 & \\
\hline DS out- 3 & & & 0 & \\
\hline DS out-4 & & & 0 & \\
\hline Other & & & 0 & $0.0 \%$ \\
\hline
\end{tabular}

\begin{tabular}{|lcccc|}
\hline Test Chamber Environment & & & \\
\hline Initial Conditions & $\mathrm{RH}(\%)$ & Temperature $\left({ }^{\circ} \mathrm{C}\right)$ & & Atm. P. (mbar) \\
1-HUM/TMP-BDST & 91.6 & 26.5 & P-IN-BDST & 854 \\
2-HUM/TMP-BDST & 90.7 & 26.5 & & \\
\hline Final Conditions & $\mathrm{RH}(\%)$ & Temperature $\left({ }^{\circ} \mathrm{C}\right)$ & & Atm. P. (mbar) \\
1-HUM/TMP-BDST & 93.4 & 27.9 & P-IN-BDST & 856 \\
2-HUM/TMP-BDST & 93.2 & 27.6 & & \\
\hline
\end{tabular}


Single Patch q(splash) Test Summary and Mass Balance - Rough Surface

Test: Patch 6, Patch center, 16.5

\begin{tabular}{|c|c|c|c|c|}
\hline & Initial & Final & Difference & $\%$ of Input \\
\hline Water Input & 788.3 & 266.5 & 521.8 & $100 \%$ \\
\hline Evap & 99.6 & 99.2 & 0.4 & \\
\hline G1-1 & 66.3 & 292.9 & 226.6 & \multirow[b]{5}{*}{$76.4 \%$} \\
\hline$\overline{G 1-2}$ & 58.2 & 230.1 & 171.9 & \\
\hline G1-3 & & & 0 & \\
\hline G1-4 & & & 0 & \\
\hline SS-1 & & & 0 & \\
\hline G2-1 & & & 0 & \multirow[b]{5}{*}{$0.0 \%$} \\
\hline G2-2 & & & 0 & \\
\hline G2-3 & & & 0 & \\
\hline G2-4 & & & 0 & \\
\hline SS-2 & & & 0 & \\
\hline G3-1 & 49.6 & 50.6 & 1 & \multirow[b]{5}{*}{$0.2 \%$} \\
\hline G3-2 & & & 0 & \\
\hline G3-3 & & & 0 & \\
\hline G3-4 & & & 0 & \\
\hline SS-3 & & & 0 & \\
\hline G4-1 & & & 0 & \multirow[b]{5}{*}{$0.0 \%$} \\
\hline G4-2 & & & 0 & \\
\hline G4-3 & & & 0 & \\
\hline G4-4 & & & 0 & \\
\hline SS-4 & & & 0 & \\
\hline$\overline{B 1}$ & & & 0 & $0.0 \%$ \\
\hline B2 & & & 0 & $0.0 \%$ \\
\hline B3 & & & 0 & $0.0 \%$ \\
\hline B4 & 107.5 & 108.1 & 0.6 & $0.1 \%$ \\
\hline B5 & 109.3 & 110.7 & 1.4 & $0.3 \%$ \\
\hline B6 & 109.8 & 110.6 & 0.8 & $0.2 \%$ \\
\hline DS in-1 & & & 0 & \multirow[b]{2}{*}{$0.0 \%$} \\
\hline DS in-2 & & & 0 & \\
\hline DS out-1 & 65.6 & 110.7 & 45.1 & \multirow[b]{4}{*}{$9.3 \%$} \\
\hline DS out- 2 & 8.4 & 12.0 & 3.6 & \\
\hline DS out-3 & & & 0 & \\
\hline DS out-4 & & & 0 & \\
\hline Other & & & 0 & $0.0 \%$ \\
\hline
\end{tabular}

Test Date: $\quad 7 / 1 / 02$

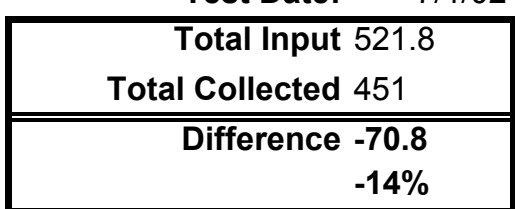

\begin{tabular}{|lr|}
\hline \multicolumn{2}{|c|}{ Evaporation } \\
Evap Pan Tare Wt, g & 66.7 \\
Evaporation, $\mathrm{g}$ & 0.4 \\
\hline \% Evaporation & $\mathbf{1 . 2 2} \%$ \\
\hline
\end{tabular}

\begin{tabular}{|lr|}
\hline \multicolumn{2}{|c|}{ Mass Flow Rate } \\
Test Duration, min & 108 \\
Flow Rate, g/min & 4.83 \\
& \\
\hline
\end{tabular}

Reference Towel Mass

Initial Mass $(\mathrm{g}) \quad 8.118$

Final Mass $(\mathrm{g}) \quad 8.757$

\begin{tabular}{lr}
\hline Difference $(\mathrm{g})$ & 0.64 \\
\hline
\end{tabular}

\begin{tabular}{|lcccc|}
\hline Test Chamber Environment & & & \\
\hline Initial Conditions & $\mathrm{RH}(\%)$ & Temperature $\left({ }^{\circ} \mathrm{C}\right)$ & & Atm. P. (mbar) \\
1-HUM/TMP-BDST & 85.3 & 26.4 & P-IN-BDST & 862 \\
2-HUM/TMP-BDST & 85.5 & 26.2 & & \\
\hline Final Conditions & $\mathrm{RH}(\%)$ & Temperature $\left({ }^{\circ} \mathrm{C}\right)$ & & Atm. P. (mbar) \\
1-HUM/TMP-BDST & 97.3 & 29.7 & P-IN-BDST & 862 \\
2-HUM/TMP-BDST & 94.5 & 29.4 & & \\
\hline
\end{tabular}

SN-M\&O-SCI-043-V2, pages 97-98 
Single Patch q(film) Test Summary and Mass Balance - Rough Surface

Test: Patch 2, centerline, $16.5^{\circ}$

Test Date: $\quad 7 / 8 / 02$

\begin{tabular}{|c|c|c|c|c|c|}
\hline & Initial (g) & Final (g) & Difference (g) & $\%$ of Input & \multirow{2}{*}{$\begin{array}{r}\text { Total Input (g) } 308.0 \\
\text { Total Collected }(\mathrm{g}) 268.6\end{array}$} \\
\hline Water Input & 28.7 & -279.3 & 308 & $100 \%$ & \\
\hline Evap & 117.5 & 117.6 & -0.1 & & \multirow{2}{*}{$\begin{array}{r}\text { Difference }(\mathrm{g})-39.4 \\
-13 \%\end{array}$} \\
\hline G1-1 & 64.4 & 66.0 & 1.6 & \multirow[b]{5}{*}{$1.4 \%$} & \\
\hline G1-2 & 48.8 & 51.4 & 2.6 & & \\
\hline G1-3 & & & 0 & & Evaporation \\
\hline G1-4 & & & 0 & & Evap Pan Tare Wt (g) \\
\hline SS-1 & & & 0 & & Evaporation (g) \\
\hline G2-1 & & & 0 & & \% Evaporation \\
\hline G2-2 & & & 0 & & \\
\hline G2-3 & & & 0 & & \\
\hline G2-4 & & & 0 & & Mass Flow Rate \\
\hline SS-2 & & & 0 & $0.0 \%$ & Test Duration (min) \\
\hline G3-1 & & & 0 & & Flow Rate (g/min) $\quad 5.13$ \\
\hline G3-2 & 41.5 & 42.3 & 0.8 & & \\
\hline G3-3 & & & 0 & & \\
\hline G3-4 & & & 0 & & $\begin{array}{l}\text { Reference Towel Mass } \\
\end{array}$ \\
\hline SS-3 & & & 0 & $0.3 \%$ & Initial Mass $(\mathrm{g})$ \\
\hline G4-1 & & & 0 & & Final Mass $(\mathrm{g})$ \\
\hline G4-2 & & & 0 & & Difference (g) \\
\hline
\end{tabular}

\begin{tabular}{|c|c|c|c|c|}
\hline G4-3 & & & 0 & \multirow[b]{3}{*}{$0.0 \%$} \\
\hline G4-4 & & & 0 & \\
\hline SS-4 & & & 0 & \\
\hline $\mathrm{B1}$ & & & 0 & $0.0 \%$ \\
\hline B2 & 107.9 & 343.1 & 235.2 & $76.4 \%$ \\
\hline B3 & & & 0 & $0.0 \%$ \\
\hline B4 & 107.6 & 107.8 & 0.2 & $0.1 \%$ \\
\hline$\overline{B 5}$ & 109.7 & 110.3 & 0.6 & $0.2 \%$ \\
\hline B6 & 110.7 & 111.7 & 1 & $0.3 \%$ \\
\hline DS in-1 & & & 0 & \multirow[b]{2}{*}{$0.0 \%$} \\
\hline DS in-2 & & & 0 & \\
\hline DS out-1 & 63.9 & 90.5 & 26.6 & \multirow[b]{4}{*}{$8.6 \%$} \\
\hline DS out- 2 & & & 0 & \\
\hline DS out-3 & & & 0 & \\
\hline DS out- 4 & & & 0 & \\
\hline Other & & & 0 & $0.0 \%$ \\
\hline
\end{tabular}

\begin{tabular}{|lcccc|}
\hline \multicolumn{4}{l}{ Test Chamber Environment } \\
\hline Initial Conditions & $\mathrm{RH}(\%)$ & Temperature $\left({ }^{\circ} \mathrm{C}\right)$ & Atm. P. (mbar) \\
1-HUM/TMP-BDST & 95.3 & 27.9 & P-IN-BDST & 869 \\
2-HUM/TMP-BDST & 91.4 & 28.2 & & \\
\hline Final Conditions & $\mathrm{RH}(\%)$ & Temperature $\left({ }^{\circ} \mathrm{C}\right)$ & & Atm. P. (mbar) \\
1-HUM/TMP-BDST & 97.5 & 29.6 & P-IN-BDST & 865 \\
2-HUM/TMP-BDST & 93.8 & 29.5 & & \\
\hline
\end{tabular}


Single Patch q(film) Test Summary and Mass Balance - Rough Surface

Test: Patch 2, centerline $10.5 \mathrm{~cm}$ forward from crown, Patch 6 covered

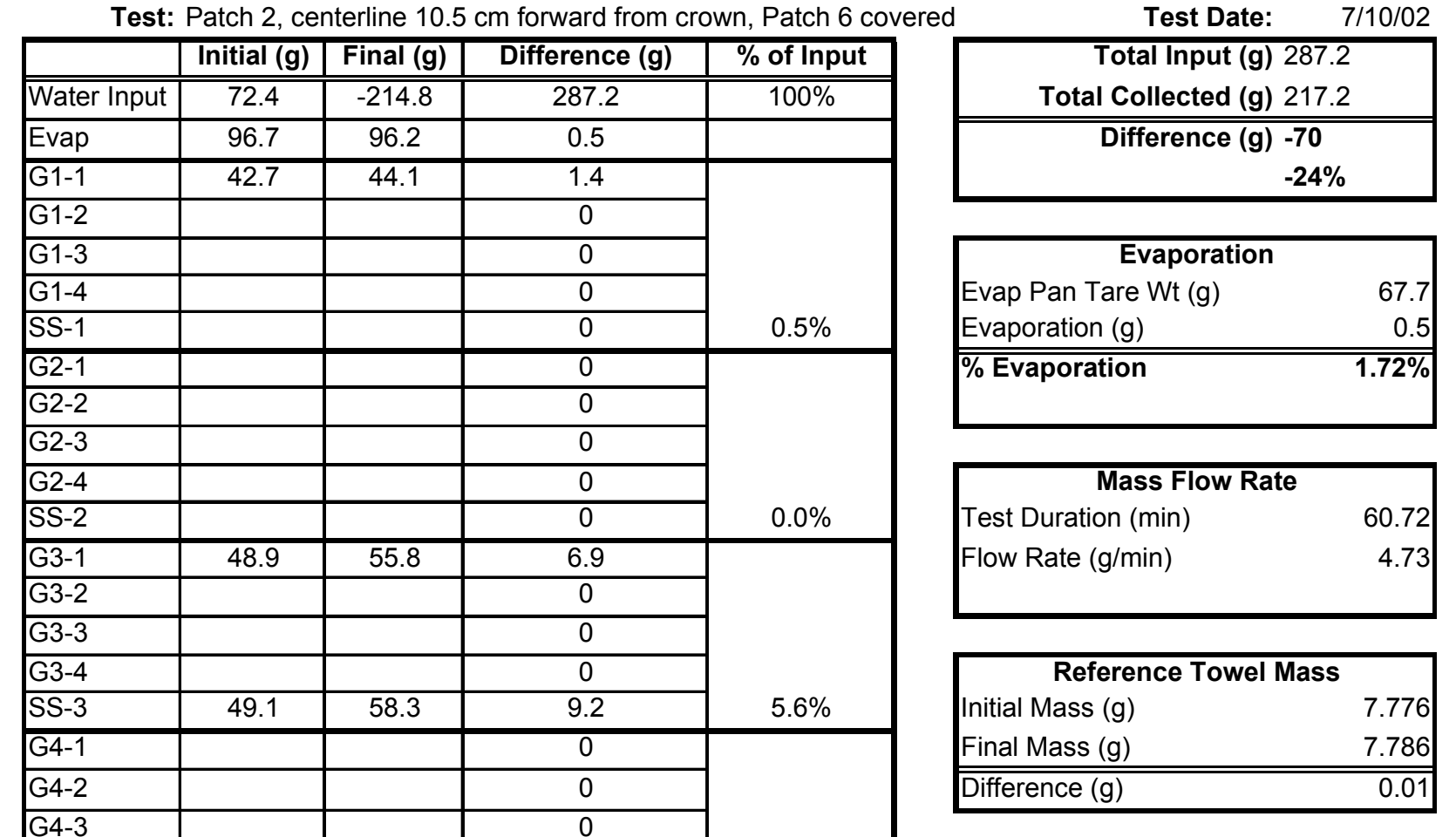

\begin{tabular}{|c|c|c|c|c|}
\hline G4-3 & & & 0 & \multirow[b]{3}{*}{$0.0 \%$} \\
\hline G4-4 & & & 0 & \\
\hline SS-4 & & & 0 & \\
\hline B1 & & & 0 & $0.0 \%$ \\
\hline B2 & 107.7 & 257.9 & 150.2 & $52.3 \%$ \\
\hline B3 & & & 0 & $0.0 \%$ \\
\hline B4 & 106.0 & 106.1 & 0.1 & $0.0 \%$ \\
\hline B5 & 109.3 & 109.4 & 0.1 & $0.0 \%$ \\
\hline $\mathrm{B} 6$ & & & 0 & $0.0 \%$ \\
\hline DS in-1 & 48.5 & 61.1 & 12.6 & \multirow[b]{2}{*}{$4.4 \%$} \\
\hline DS in-2 & & & 0 & \\
\hline DS out-1 & 64.6 & 101.3 & 36.7 & \multirow[b]{4}{*}{$12.8 \%$} \\
\hline DS out-2 & & & 0 & \\
\hline DS out-3 & & & 0 & \\
\hline DS out-4 & & & 0 & \\
\hline Other & & & 0 & $0.0 \%$ \\
\hline
\end{tabular}

\begin{tabular}{|lcccc|}
\hline \multicolumn{4}{l}{ Test Chamber Environment } \\
\hline Initial Conditions & $\mathrm{RH}(\%)$ & Temperature $\left({ }^{\circ} \mathrm{C}\right)$ & Atm. P. (mbar) \\
1-HUM/TMP-BDST & 88.1 & 20.4 & P-IN-BDST & 860 \\
2-HUM/TMP-BDST & 90.5 & 20.5 & & \\
\hline Final Conditions & $\mathrm{RH}(\%)$ & Temperature $\left({ }^{\circ} \mathrm{C}\right)$ & Atm. P. (mbar) \\
1-HUM/TMP-BDST & 89.2 & 21.6 & P-IN-BDST & 859 \\
2-HUM/TMP-BDST & 85.1 & 22.1 & & \\
\hline
\end{tabular}


Single Patch q(film) Test Summary and Mass Balance - Rough Surface

Test: Patch 2, $13.5 \mathrm{~cm}$ right of patch center $10.5 \mathrm{~cm}$ forward from crowı

\begin{tabular}{|l|c|c|c|c|}
\hline & Initial (g) & Final (g) & Difference (g) & \% of Input \\
\hline \hline Water Input & 53.5 & -234.9 & 288.4 & $100 \%$ \\
\hline Evap & 96.2 & 95.1 & 1.1 & \\
\hline G1-1 & 57.5 & 131.3 & 73.8 & \\
\hline
\end{tabular}

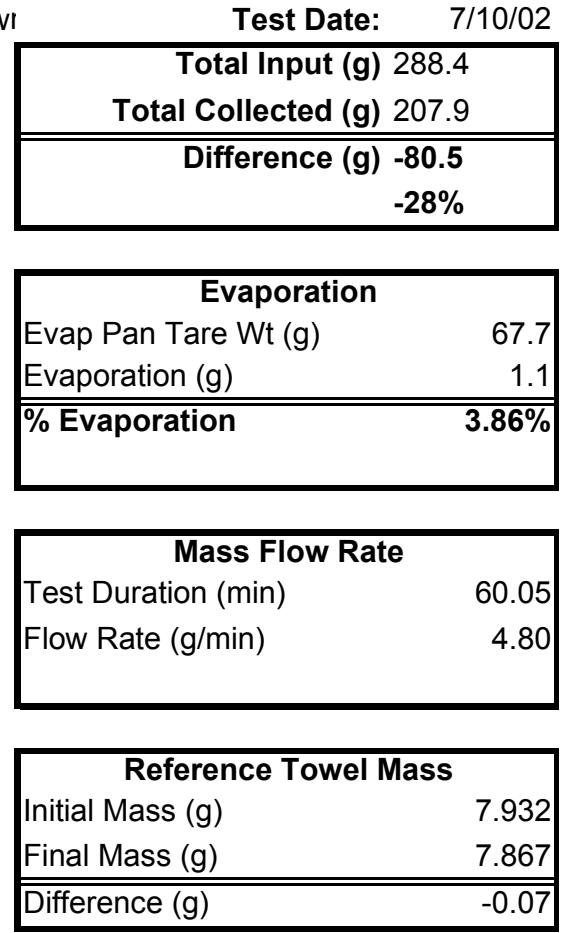

\begin{tabular}{|c|c|c|c|c|}
\hline G4-2 & & & 0 & \multirow[b]{4}{*}{$0.0 \%$} \\
\hline G4-3 & & & 0 & \\
\hline G4-4 & & & 0 & \\
\hline SS-4 & & & 0 & \\
\hline$B 1$ & & & 0 & $0.0 \%$ \\
\hline B2 & 108.2 & 172.2 & 64 & $22.2 \%$ \\
\hline B3 & & & 0 & $0.0 \%$ \\
\hline B4 & & & 0 & $0.0 \%$ \\
\hline B5 & 109.5 & 109.5 & 0 & $0.0 \%$ \\
\hline B6 & & & 0 & $0.0 \%$ \\
\hline DS in-1 & 41.2 & 45.6 & 4.4 & \multirow[b]{2}{*}{$1.5 \%$} \\
\hline DS in-2 & & & 0 & \\
\hline DS out- 1 & 64.9 & 114.6 & 49.7 & \multirow[b]{4}{*}{$17.2 \%$} \\
\hline DS out-2 & & & 0 & \\
\hline DS out-3 & & & 0 & \\
\hline DS out-4 & & & 0 & \\
\hline Other & & & 0 & $0.0 \%$ \\
\hline
\end{tabular}

\begin{tabular}{|lcccc|}
\hline \multicolumn{4}{l}{ Test Chamber Environment } & \multicolumn{4}{l|}{} \\
\hline Initial Conditions & $\mathrm{RH}(\%)$ & Temperature $\left({ }^{\circ} \mathrm{C}\right)$ & Atm. P. (mbar) \\
1-HUM/TMP-BDST & 91.1 & 23 & P-IN-BDST & 853 \\
2-HUM/TMP-BDST & 90.4 & 22.9 & & \\
\hline Final Conditions & $\mathrm{RH}(\%)$ & Temperature $\left({ }^{\circ} \mathrm{C}\right)$ & Atm. P. (mbar) \\
1-HUM/TMP-BDST & 90.2 & 23.9 & P-IN-BDST & 847 \\
2-HUM/TMP-BDST & 85.4 & 24.3 & & \\
\hline
\end{tabular}


Single Patch q(film) Test Summary and Mass Balance - Rough Surface

Test: Patch 2, $13.5 \mathrm{cn}$ right of center, 33

\begin{tabular}{|c|c|c|c|c|}
\hline & Initial & Final & Difference & $\%$ of Input \\
\hline Water Input & 7740.3 & 432.3 & 308 & $100 \%$ \\
\hline Evap & 101.2 & 101.0 & 0.2 & \\
\hline$\overline{\mathrm{G} 1-1}$ & 56.6 & 78.3 & 21.7 & \multirow[b]{5}{*}{$7.0 \%$} \\
\hline G1-2 & & & 0 & \\
\hline $\mathrm{G} 1-3$ & & & 0 & \\
\hline $\mathrm{G} 1-4$ & & & 0 & \\
\hline SS-1 & & & 0 & \\
\hline $\bar{G} 2-1$ & & & 0 & \multirow[b]{5}{*}{$0.0 \%$} \\
\hline G2-2 & & & 0 & \\
\hline G2-3 & & & 0 & \\
\hline G2-4 & & & 0 & \\
\hline SS-2 & & & 0 & \\
\hline G3-1 & & & 0 & \multirow[b]{5}{*}{$0.0 \%$} \\
\hline G3-2 & & & 0 & \\
\hline G3-3 & & & 0 & \\
\hline G3-4 & & & 0 & \\
\hline SS-3 & & & 0 & \\
\hline G4-1 & & & 0 & \multirow[b]{5}{*}{$0.0 \%$} \\
\hline G4-2 & & & 0 & \\
\hline G4-3 & & & 0 & \\
\hline G4-4 & & & 0 & \\
\hline SS-4 & & & 0 & \\
\hline $\mathrm{B} 1$ & & & 0 & $0.0 \%$ \\
\hline B2 & 107.7 & 287.2 & 179.5 & $58.3 \%$ \\
\hline B2-2 & 48.3 & 99.0 & 50.7 & $16.5 \%$ \\
\hline B4 & & & 0 & $0.0 \%$ \\
\hline$\overline{B 5}$ & 109.2 & 110.1 & 0.9 & $0.3 \%$ \\
\hline B6 & & & 0 & $0.0 \%$ \\
\hline DS in-1 & & & 0 & \multirow[b]{2}{*}{$0.0 \%$} \\
\hline DS in-2 & & & 0 & \\
\hline DS out-1 & 56.8 & 68.4 & 11.6 & \multirow[b]{4}{*}{$3.8 \%$} \\
\hline DS out-2 & & & 0 & \\
\hline DS out-3 & & & 0 & \\
\hline DS out-4 & & & 0 & \\
\hline Other & & & 0 & $0.0 \%$ \\
\hline
\end{tabular}

Test Date: $\quad 7 / 3 / 02$
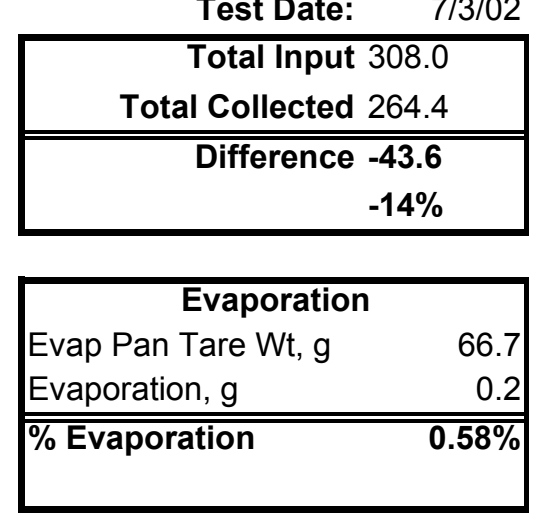

\begin{tabular}{|lr|}
\hline \multicolumn{2}{|c|}{ Mass Flow Rate } \\
Test Duration, min & 60 \\
Flow Rate, g/min & 5.13 \\
\hline
\end{tabular}

\begin{tabular}{|lr|}
\hline \multicolumn{2}{|c|}{ Reference Towel Mass } \\
Initial Mass (g) & 7.808 \\
Final Mass $(\mathrm{g})$ & 8.066 \\
\hline \hline Difference $(\mathrm{g})$ & 0.26 \\
\hline
\end{tabular}

\begin{tabular}{|lcccc|}
\hline Test Chamber Environment & & & \\
\hline Initial Conditions & $\mathrm{RH}(\%)$ & Temperature $\left({ }^{\circ} \mathrm{C}\right)$ & & Atm. P. (mbar) \\
1-HUM/TMP-BDST & 89.4 & 26.3 & P-IN-BDST & 858 \\
2-HUM/TMP-BDST & 90.7 & 25.8 & & \\
\hline Final Conditions & $\mathrm{RH}(\%)$ & Temperature $\left({ }^{\circ} \mathrm{C}\right)$ & & Atm. P. (mbar) \\
1-HUM/TMP-BDST & 94.5 & 27.9 & P-IN-BDST & 867 \\
2-HUM/TMP-BDST & 94.6 & 27.6 & & \\
\hline
\end{tabular}


Single Patch q(film) Test Summary and Mass Balance - Rough Surface

Test: Patch 2, Centerline, 33

\begin{tabular}{|c|c|c|c|c|}
\hline & Initial & Final & Difference & $\%$ of Input \\
\hline Water Input & 705.7 & $\overline{398.3}$ & 307.4 & $100 \%$ \\
\hline Evap & 103.0 & 102.7 & 0.3 & \\
\hline G1-1 & 64.1 & 80.5 & 16.4 & \multirow[b]{5}{*}{$5.3 \%$} \\
\hline G1-2 & & & 0 & \\
\hline G1-3 & & & 0 & \\
\hline G1-4 & & & 0 & \\
\hline SS-1 & & & 0 & \\
\hline G2-1 & & & 0 & \multirow[b]{5}{*}{$0.0 \%$} \\
\hline G2-2 & & & 0 & \\
\hline G2-3 & & & 0 & \\
\hline G2-4 & & & 0 & \\
\hline SS-2 & & & 0 & \\
\hline G3-1 & & & 0 & \multirow[b]{5}{*}{$0.0 \%$} \\
\hline G3-2 & & & 0 & \\
\hline G3-3 & & & 0 & \\
\hline G3-4 & & & 0 & \\
\hline SS-3 & & & 0 & \\
\hline G4-1 & & & 0 & \multirow[b]{5}{*}{$0.0 \%$} \\
\hline G4-2 & & & 0 & \\
\hline G4-3 & & & 0 & \\
\hline G4-4 & & & 0 & \\
\hline SS-4 & & & 0 & \\
\hline $\mathrm{B} 1$ & & & 0 & $0.0 \%$ \\
\hline B2 & 107.4 & 350.9 & 243.5 & $79.2 \%$ \\
\hline B3 & & & 0 & $0.0 \%$ \\
\hline B4 & 107.3 & 107.6 & 0.3 & $0.1 \%$ \\
\hline B5 & 109.2 & 109.8 & 0.6 & $0.2 \%$ \\
\hline B6 & & & 0 & $0.0 \%$ \\
\hline DS in-1 & & & 0 & \multirow[b]{2}{*}{$0.0 \%$} \\
\hline DS in-2 & & & 0 & \\
\hline DS out-1 & 64.8 & 75.1 & 10.3 & \multirow[b]{4}{*}{$3.4 \%$} \\
\hline DS out-2 & & & 0 & \\
\hline DS out-3 & & & 0 & \\
\hline DS out-4 & & & 0 & \\
\hline Other & & & 0 & $0.0 \%$ \\
\hline
\end{tabular}
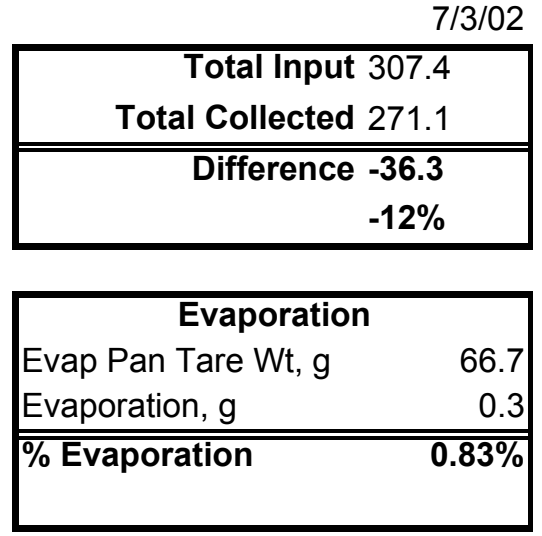

\begin{tabular}{|lr|}
\hline \multicolumn{2}{|c|}{ Mass Flow Rate } \\
Test Duration, min & 60 \\
Flow Rate, g/min & 5.12 \\
\hline
\end{tabular}

\section{Reference Towel Mass}

Initial Mass $(\mathrm{g})$

Final Mass $(\mathrm{g}) \quad 8.239$

\begin{tabular}{lr}
\hline Difference $(\mathrm{g})$ & 0.59 \\
\hline
\end{tabular}

\begin{tabular}{|lcccc|}
\hline \multicolumn{7}{l}{ Test Chamber Environment } & & & \\
\hline Initial Conditions & $\mathrm{RH}(\%)$ & Temperature $\left({ }^{\circ} \mathrm{C}\right)$ & & Atm. P. (mbar) \\
1-HUM/TMP-BDST & 86.8 & 26.3 & P-IN-BDST & 862 \\
2-HUM/TMP-BDST & 89.3 & 26.2 & & \\
\hline Final Conditions & $\mathrm{RH}(\%)$ & Temperature $\left({ }^{\circ} \mathrm{C}\right)$ & & Atm. P. (mbar) \\
1-HUM/TMP-BDST & 94.1 & 27.5 & P-IN-BDST & 873 \\
2-HUM/TMP-BDST & 94.2 & 27.2 & & \\
\hline
\end{tabular}


Single Patch q(film) Test Summary and Mass Balance - Rough Surface

Test: Patch2, $13.5 \mathrm{~cm}$ right of centerline, 16.5

\begin{tabular}{|c|c|c|c|c|}
\hline & Initial & Final & Difference & $\%$ of Input \\
\hline Water Input & $\overline{7767.1}$ & 460.6 & 306.5 & $100 \%$ \\
\hline Evap & 99.5 & 99.4 & 0.1 & \\
\hline G1-1 & 64.2 & 144.0 & 79.8 & \multirow[b]{5}{*}{$26.0 \%$} \\
\hline$\overline{G 1-2}$ & & & 0 & \\
\hline G1-3 & & & 0 & \\
\hline $\mathrm{G} 1-4$ & & & 0 & \\
\hline SS-1 & & & 0 & \\
\hline$\overline{\mathrm{G} 2-1}$ & & & 0 & \multirow[b]{5}{*}{$0.0 \%$} \\
\hline G2-2 & & & 0 & \\
\hline G2-3 & & & 0 & \\
\hline$\overline{\mathrm{G} 2-4}$ & & & 0 & \\
\hline SS-2 & & & 0 & \\
\hline$\overline{\text { G3-1 }}$ & 42.1 & 42.3 & 0.2 & \multirow[b]{5}{*}{$0.1 \%$} \\
\hline G3-2 & & & 0 & \\
\hline G3-3 & & & 0 & \\
\hline G3-4 & & & 0 & \\
\hline SS-3 & & & 0 & \\
\hline G4-1 & & & 0 & \multirow[b]{5}{*}{$0.0 \%$} \\
\hline G4-2 & & & 0 & \\
\hline G4-3 & & & 0 & \\
\hline G4-4 & & & 0 & \\
\hline SS-4 & & & 0 & \\
\hline B1 & & & 0 & $0.0 \%$ \\
\hline B2 & 107.6 & 232.4 & 124.8 & $40.7 \%$ \\
\hline B3 & & & 0 & $0.0 \%$ \\
\hline B4 & 99.7 & 99.7 & 0 & $0.0 \%$ \\
\hline B5 & 109.2 & 111.1 & 1.9 & $0.6 \%$ \\
\hline B6 & 109.8 & 110.4 & 0.6 & $0.2 \%$ \\
\hline DS in-1 & & & 0 & \multirow[b]{2}{*}{$0.0 \%$} \\
\hline$\overline{D S}$ in-2 & & & 0 & \\
\hline DS out-1 & 63.8 & 105.6 & 41.8 & \multirow{4}{*}{$13.6 \%$} \\
\hline DS out- 2 & & & 0 & \\
\hline DS out-3 & & & 0 & \\
\hline DS out-4 & & & 0 & \\
\hline Other & & & 0 & $0.0 \%$ \\
\hline
\end{tabular}

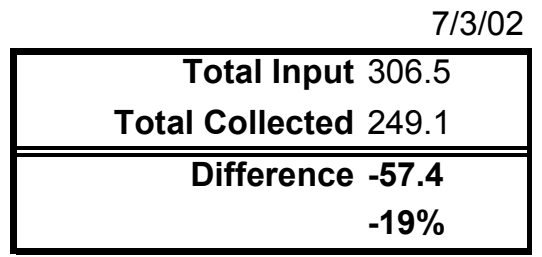

\begin{tabular}{|lr|}
\hline \multicolumn{2}{|c|}{ Evaporation } \\
Evap Pan Tare Wt, g & 66.7 \\
Evaporation, g & 0.1 \\
\hline \% Evaporation & $\mathbf{0 . 3 0 \%}$ \\
\hline
\end{tabular}

\begin{tabular}{|lr|}
\hline \multicolumn{2}{|c|}{ Mass Flow Rate } \\
Test Duration, min & 60 \\
Flow Rate, g/min & 5.11 \\
\hline
\end{tabular}

\begin{tabular}{|lr|}
\hline \multicolumn{2}{|c|}{ Reference Towel Mass } \\
Initial Mass $(\mathrm{g})$ & 7.631 \\
Final Mass $(\mathrm{g})$ & 7.839 \\
\hline \hline Difference $(\mathrm{g})$ & 0.21 \\
\hline
\end{tabular}

Test Chamber Environment

\begin{tabular}{|lcccc|}
\hline Initial Conditions & $\mathrm{RH}(\%)$ & Temperature $\left({ }^{\circ} \mathrm{C}\right)$ & & Atm. P. (mbar) \\
1-HUM/TMP-BDST & 91.4 & 27.4 & P-IN-BDST & 860 \\
2-HUM/TMP-BDST & 90.4 & 27.5 & & \\
\hline Final Conditions & $\mathrm{RH}(\%)$ & Temperature $\left({ }^{\circ} \mathrm{C}\right)$ & & Atm. P. (mbar) \\
1-HUM/TMP-BDST & 93.2 & 29 & P-IN-BDST & 861 \\
2-HUM/TMP-BDST & 94.1 & 28.5 & & \\
\hline
\end{tabular}


Single Patch q(film) Test Summary and Mass Balance - Rough Surface

Test: Patch 4, $13.5 \mathrm{~cm}$ right of patch center, $16.5^{\circ}$

Test Date: $\quad 7 / 8 / 02$

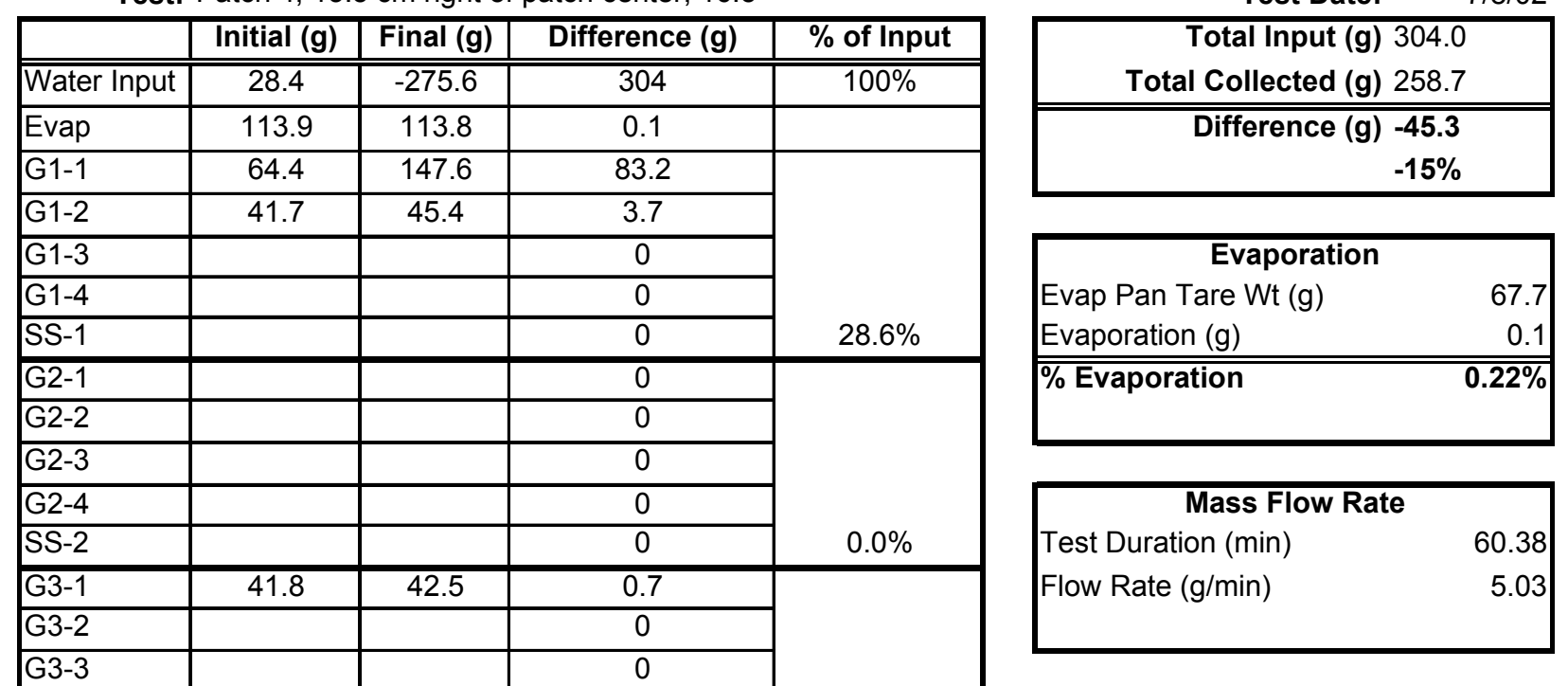

\begin{tabular}{|c|c|c|c|c|}
\hline & & & & \multirow[b]{3}{*}{$0.2 \%$} \\
\hline G3-4 & & & 0 & \\
\hline SS-3 & & & 0 & \\
\hline G4-1 & & & $\overline{0}$ & \multirow[b]{5}{*}{$0.0 \%$} \\
\hline G4-2 & & & 0 & \\
\hline G4-3 & & & 0 & \\
\hline G4-4 & & & 0 & \\
\hline SS-4 & & & 0 & \\
\hline $\mathrm{B} 1$ & & & 0 & $0.0 \%$ \\
\hline B2 & 107.6 & 108.2 & 0.6 & $0.2 \%$ \\
\hline B3 & & & 0 & $0.0 \%$ \\
\hline B4 & 107.2 & 239.3 & 132.1 & $43.5 \%$ \\
\hline B5 & 109.1 & 109.8 & 0.7 & $0.2 \%$ \\
\hline B6 & 109.8 & 110.8 & 1 & $0.3 \%$ \\
\hline DS in-1 & 40.9 & 42.1 & 1.2 & \multirow[b]{2}{*}{$0.4 \%$} \\
\hline DS in-2 & & & 0 & \\
\hline DS out-1 & 63.6 & 99.1 & 35.5 & \multirow[b]{4}{*}{$11.7 \%$} \\
\hline DS out-2 & & & 0 & \\
\hline DS out-3 & & & 0 & \\
\hline DS out-4 & & & 0 & \\
\hline Other & & & 0 & $0.0 \%$ \\
\hline
\end{tabular}

\begin{tabular}{|lr|}
\hline \multicolumn{2}{|c|}{ Reference Towel Mass } \\
Initial Mass $(\mathrm{g})$ & 7.719 \\
Final Mass $(\mathrm{g})$ & 8.28 \\
\hline \hline Difference $(\mathrm{g})$ & 0.56 \\
\hline
\end{tabular}

\begin{tabular}{|lcccc|}
\hline \multicolumn{4}{ll}{ Test Chamber Environment } \\
\hline Initial Conditions & $\mathrm{RH}(\%)$ & Temperature $\left({ }^{\circ} \mathrm{C}\right)$ & Atm. P. (mbar) \\
1-HUM/TMP-BDST & 82.6 & 23.9 & P-IN-BDST & 855 \\
2-HUM/TMP-BDST & 87.6 & 23.7 & & \\
\hline Final Conditions & $\mathrm{RH}(\%)$ & Temperature $\left({ }^{\circ} \mathrm{C}\right)$ & Atm. P. (mbar) \\
1-HUM/TMP-BDST & 94.8 & 28.4 & P-IN-BDST & 852 \\
2-HUM/TMP-BDST & 93.5 & 28.3 & & \\
\hline
\end{tabular}


Single Patch q(film) Test Summary and Mass Balance - Rough Surface

Test: Patch 4,centerline, $16.5^{\circ}$

\begin{tabular}{|c|c|c|c|c|}
\hline & Initial (g) & Final (g) & Difference $(\mathrm{g})$ & $\%$ of Input \\
\hline Water Input & -18.3 & -320.0 & 301.7 & $100 \%$ \\
\hline Evap & 88.5 & 88.0 & 0.5 & \\
\hline$\overline{G 1-1}$ & 42.5 & 42.6 & 0.1 & \multirow[b]{5}{*}{$1.5 \%$} \\
\hline G1-2 & 41.5 & 45.8 & 4.3 & \\
\hline G1-3 & & & 0 & \\
\hline G1-4 & & & 0 & \\
\hline SS-1 & & & 0 & \\
\hline G2-1 & & & 0 & \multirow[b]{5}{*}{$0.0 \%$} \\
\hline G2-2 & & & 0 & \\
\hline G2-3 & & & 0 & \\
\hline G2-4 & & & 0 & \\
\hline SS-2 & & & 0 & \\
\hline G3-1 & & & 0 & \multirow[b]{5}{*}{$0.1 \%$} \\
\hline G3-2 & & & 0 & \\
\hline G3-3 & & & 0 & \\
\hline G3-4 & & & 0 & \\
\hline SS-3 & 41.7 & 42.1 & 0.4 & \\
\hline G4-1 & & & 0 & \multirow[b]{5}{*}{$0.0 \%$} \\
\hline G4-2 & & & 0 & \\
\hline G4-3 & & & 0 & \\
\hline G4-4 & & & 0 & \\
\hline SS-4 & & & 0 & \\
\hline B1 & 105.9 & 106.2 & 0.3 & $0.1 \%$ \\
\hline $\mathrm{B} 2$ & 107.6 & 107.9 & 0.3 & $0.1 \%$ \\
\hline B3 & & & 0 & $0.0 \%$ \\
\hline B4 & 107.4 & 307.3 & 199.9 & $66.3 \%$ \\
\hline B5 & 109.1 & 109.4 & 0.3 & $0.1 \%$ \\
\hline B6 & 109.8 & 110.2 & 0.4 & $0.1 \%$ \\
\hline DS in-1 & & & 0 & \multirow[b]{2}{*}{$0.0 \%$} \\
\hline DS in-2 & & & 0 & \\
\hline DS out-1 & 64.5 & 92.1 & 27.6 & \multirow{4}{*}{$9.1 \%$} \\
\hline DS out-2 & & & 0 & \\
\hline DS out- 3 & & & 0 & \\
\hline DS out-4 & & & 0 & \\
\hline Other & & & 0 & $0.0 \%$ \\
\hline
\end{tabular}

Test Date: $\quad$ 7/9/02

\begin{tabular}{|c|}
\hline $\begin{array}{r}\text { Total Input }(\mathrm{g}) 301.7 \\
\text { Total Collected }(\mathrm{g}) 233.6\end{array}$ \\
\hline "Difference (g) -68.1 \\
\hline$-23 \%$ \\
\hline
\end{tabular}

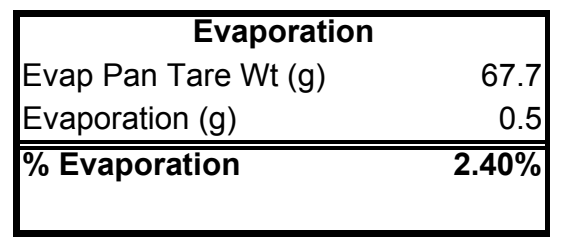

\begin{tabular}{|lr|}
\hline \multicolumn{2}{|c|}{ Mass Flow Rate } \\
Test Duration (min) & 60 \\
Flow Rate (g/min) & 5.03 \\
\hline
\end{tabular}

\begin{tabular}{|lcccc|}
\hline \multicolumn{4}{ll}{ Test Chamber Environment } \\
\hline Initial Conditions & $\mathrm{RH}(\%)$ & Temperature $\left({ }^{\circ} \mathrm{C}\right)$ & Atm. P. (mbar) \\
1-HUM/TMP-BDST & 87.1 & 20.5 & P-IN-BDST & 853 \\
2-HUM/TMP-BDST & 89.2 & 20.3 & & \\
\hline Final Conditions & $\mathrm{RH}(\%)$ & Temperature $\left({ }^{\circ} \mathrm{C}\right)$ & Atm. P. (mbar) \\
1-HUM/TMP-BDST & 91.9 & 24.1 & P-IN-BDST & 856 \\
2-HUM/TMP-BDST & 92.8 & 23.8 & & \\
\hline
\end{tabular}


Single Patch q(film) Test Summary and Mass Balance - Rough Surface

Test: Patch 4,centerline, crown

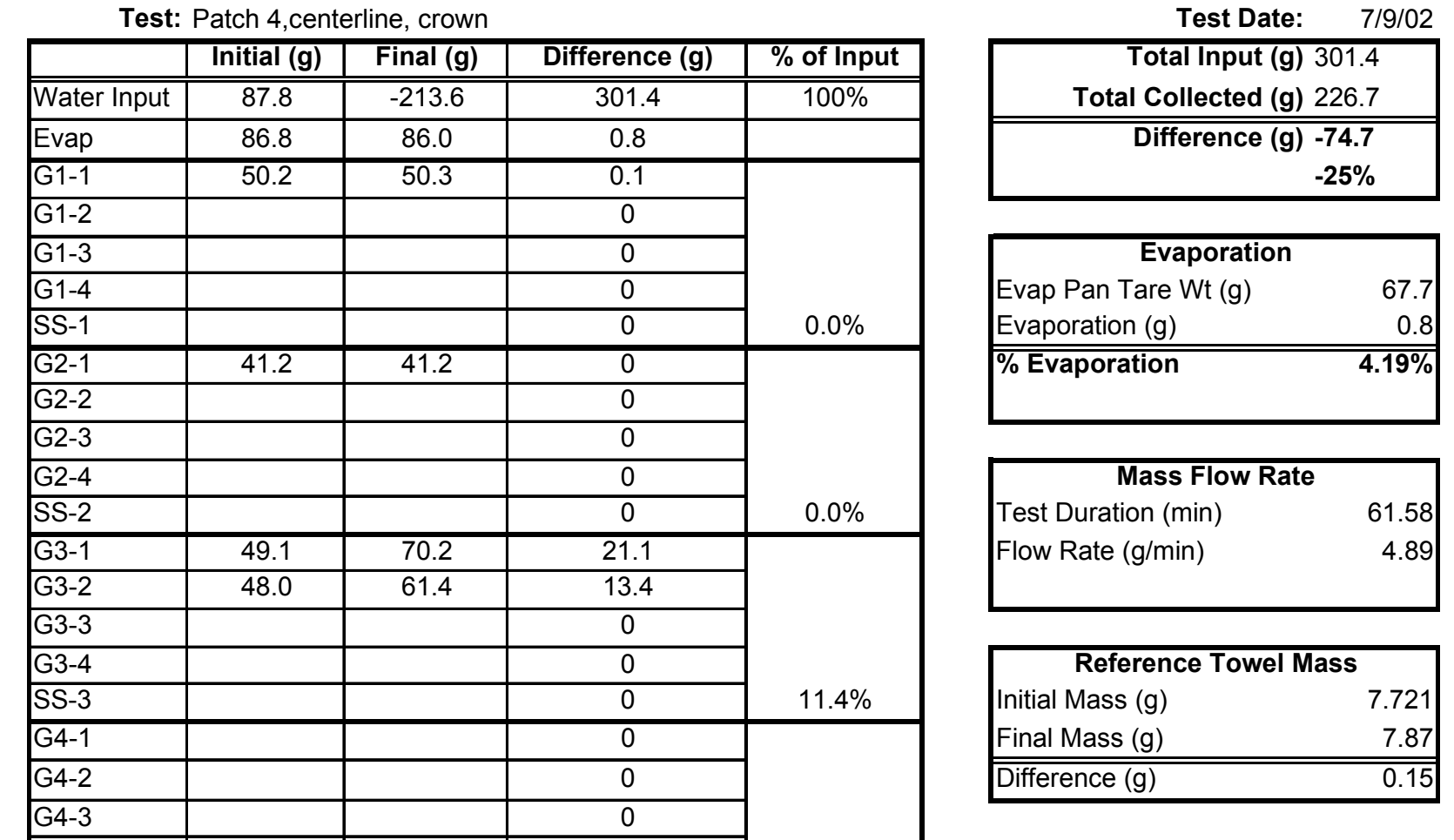

\begin{tabular}{|c|c|c|c|c|}
\hline (34-J & & & U & \multirow[b]{3}{*}{$0.0 \%$} \\
\hline G4-4 & & & 0 & \\
\hline SS-4 & & & 0 & \\
\hline B1 & 106.0 & 106.1 & 0.1 & $0.0 \%$ \\
\hline B2 & 107.7 & 107.9 & 0.2 & $0.1 \%$ \\
\hline B3 & & & 0 & $0.0 \%$ \\
\hline B4 & 107.4 & 279.1 & 171.7 & $57.0 \%$ \\
\hline B5 & & & 0 & $0.0 \%$ \\
\hline B6 & 109.9 & 110.1 & 0.2 & $0.1 \%$ \\
\hline DS in-1 & & & 0 & \multirow[b]{2}{*}{$0.0 \%$} \\
\hline DS in-2 & & & 0 & \\
\hline DS out-1 & 64.3 & 84.2 & 19.9 & \multirow[b]{4}{*}{$6.6 \%$} \\
\hline DS out-2 & & & 0 & \\
\hline DS out-3 & & & 0 & \\
\hline DS out-4 & & & 0 & \\
\hline Other & & & 0 & $0.0 \%$ \\
\hline
\end{tabular}

\begin{tabular}{|lcccc|}
\hline \multicolumn{4}{l}{ Test Chamber Environment } & \multicolumn{5}{l|}{} \\
\hline Initial Conditions & $\mathrm{RH}(\%)$ & Temperature $\left({ }^{\circ} \mathrm{C}\right)$ & Atm. P. (mbar) \\
1-HUM/TMP-BDST & 87.2 & 23.3 & P-IN-BDST & 849 \\
2-HUM/TMP-BDST & 89.8 & 23.1 & & \\
\hline Final Conditions & $\mathrm{RH}(\%)$ & Temperature $\left({ }^{\circ} \mathrm{C}\right)$ & Atm. P. (mbar) \\
1-HUM/TMP-BDST & 91.1 & 25.4 & P-IN-BDST & 853 \\
2-HUM/TMP-BDST & 91.2 & 25.3 & & \\
\hline
\end{tabular}


Single Patch q(film) Test Summary and Mass Balance - Rough Surface

Test: Patch 4, $13.5 \mathrm{~cm}$ right of patch center, crown - Test\#1

\begin{tabular}{|c|c|c|c|c|}
\hline & Initial (g) & Final (g) & Difference $(\mathrm{g})$ & $\%$ of Input \\
\hline Water Input & 16.7 & -275.5 & 292.2 & $100 \%$ \\
\hline Evap & 85.8 & 85.4 & 0.4 & \\
\hline $\mathrm{G} 1-1$ & 85.8 & 85.4 & -0.4 & \multirow[b]{5}{*}{$-0.1 \%$} \\
\hline G1-2 & & & 0 & \\
\hline G1-3 & & & 0 & \\
\hline G1-4 & & & 0 & \\
\hline SS-1 & & & 0 & \\
\hline G2-1 & & & 0 & \multirow[b]{5}{*}{$0.0 \%$} \\
\hline G2-2 & & & 0 & \\
\hline G2-3 & & & 0 & \\
\hline G2-4 & & & 0 & \\
\hline SS-2 & & & 0 & \\
\hline G3-1 & & & 0 & \multirow[b]{5}{*}{$0.0 \%$} \\
\hline G3-2 & & & 0 & \\
\hline G3-3 & & & 0 & \\
\hline G3-4 & & & 0 & \\
\hline SS-3 & & & 0 & \\
\hline G4-1 & & & 0 & \multirow[b]{5}{*}{$0.0 \%$} \\
\hline G4-2 & & & 0 & \\
\hline G4-3 & & & 0 & \\
\hline G4-4 & & & 0 & \\
\hline SS-4 & & & 0 & \\
\hline $\mathrm{B} 1$ & & & 0 & $0.0 \%$ \\
\hline $\mathrm{B} 2$ & 107.8 & 108.0 & 0.2 & $0.1 \%$ \\
\hline B3 & & & 0 & $0.0 \%$ \\
\hline B4 & 107.4 & 149.8 & 42.4 & $14.5 \%$ \\
\hline B5 & & & 0 & $0.0 \%$ \\
\hline $\mathrm{B} 6$ & 110.1 & 111.8 & 1.7 & $0.6 \%$ \\
\hline DS in-1 & 48.8 & 132.4 & 83.6 & \multirow[b]{2}{*}{$29.5 \%$} \\
\hline DS in-2 & 48.2 & 50.8 & 2.6 & \\
\hline DS out-1 & & & 0 & \multirow[b]{4}{*}{$0.0 \%$} \\
\hline DS out-2 & & & 0 & \\
\hline DS out-3 & & & 0 & \\
\hline DS out-4 & & & 0 & \\
\hline Other & & & 0 & $0.0 \%$ \\
\hline
\end{tabular}

Test Date: $\quad 7 / 9 / 02$

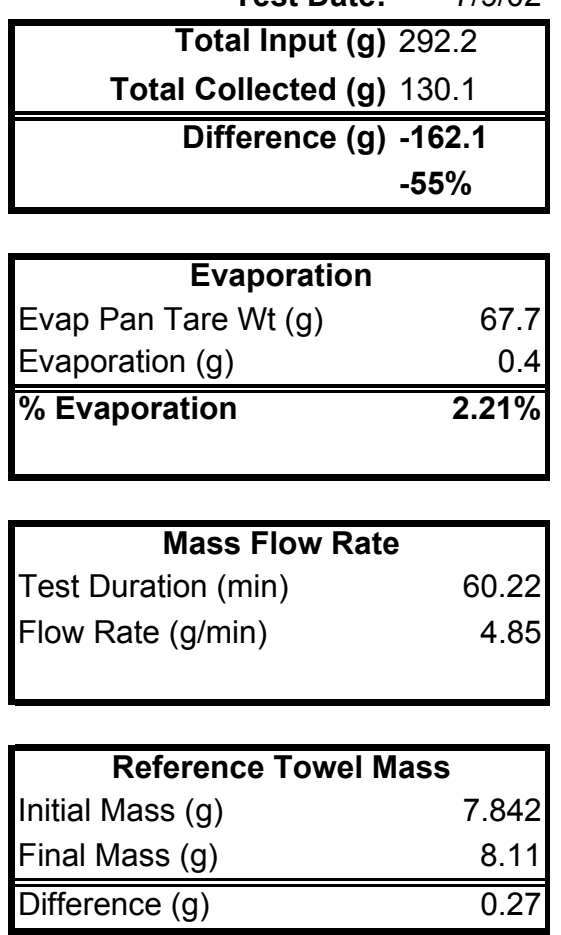

\begin{tabular}{|lcccc|}
\hline \multicolumn{5}{ll}{ Test Chamber Environment } \\
\hline Initial Conditions & $\mathrm{RH}(\%)$ & Temperature $\left({ }^{\circ} \mathrm{C}\right)$ & & Atm. P. (mbar) \\
1-HUM/TMP-BDST & 90.1 & 25.6 & P-IN-BDST & \\
2-HUM/TMP-BDST & 87.9 & 25.8 & & \\
\hline Final Conditions & $\mathrm{RH}(\%)$ & Temperature $\left({ }^{\circ} \mathrm{C}\right)$ & & Atm. P. (mbar) \\
1-HUM/TMP-BDST & 93.8 & 28.1 & P-IN-BDST & \\
2-HUM/TMP-BDST & 91.5 & 28.1 & & \\
\hline
\end{tabular}


Single Patch q(film) Test Summary and Mass Balance - Rough Surface

Test: Patch 4,13.5 cm right of patch center, crown - Test\#2

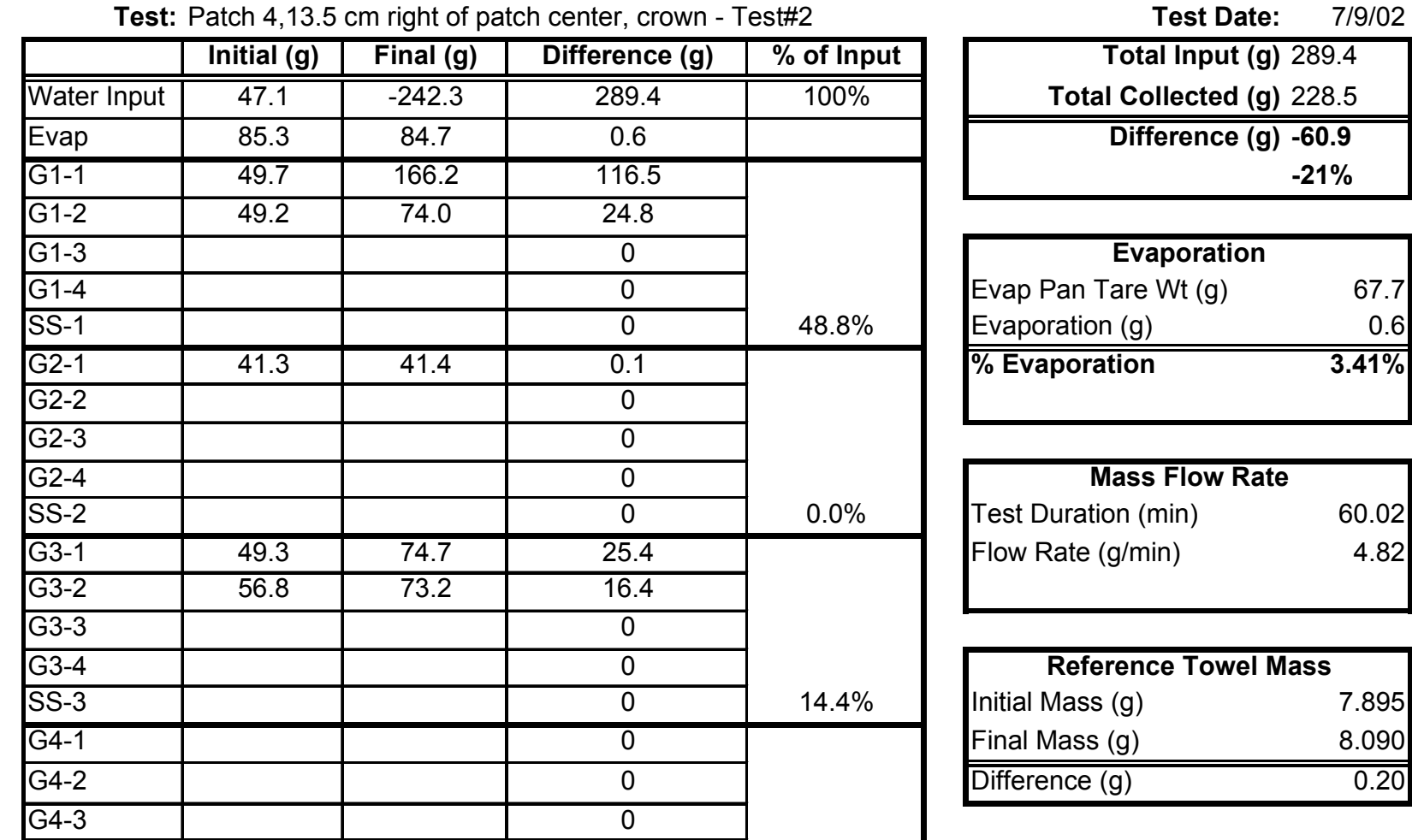

\begin{tabular}{|c|c|c|c|c|}
\hline & & & & \\
\hline G4-4 & & & 0 & \\
\hline SS-4 & & & 0 & $0.0 \%$ \\
\hline B1 & & & 0 & $0.0 \%$ \\
\hline $\mathrm{B} 2$ & 107.8 & 108.0 & 0.2 & $0.1 \%$ \\
\hline B3 & & & 0 & $0.0 \%$ \\
\hline B4 & 106.1 & 106.8 & 0.7 & $0.2 \%$ \\
\hline B5 & & & 0 & $0.0 \%$ \\
\hline B6 & 110.1 & 111.7 & 1.6 & $0.6 \%$ \\
\hline DS in-1 & & & 0 & \\
\hline DS in-2 & & & 0 & $0.0 \%$ \\
\hline DS out-1 & 64.7 & 107.5 & 42.8 & \\
\hline DS out-2 & & & 0 & \\
\hline DS out-3 & & & 0 & \\
\hline DS out-4 & & & 0 & $14.8 \%$ \\
\hline Other & & & 0 & $0.0 \%$ \\
\hline
\end{tabular}

\begin{tabular}{|lcccc|}
\hline \multicolumn{4}{l}{ Test Chamber Environment } & \multicolumn{4}{l|}{} \\
\hline Initial Conditions & $\mathrm{RH}(\%)$ & Temperature $\left({ }^{\circ} \mathrm{C}\right)$ & Atm. P. (mbar) \\
1-HUM/TMP-BDST & 89.2 & 27.5 & P-IN-BDST & 854 \\
2-HUM/TMP-BDST & 81.1 & 28 & & \\
\hline Final Conditions & $\mathrm{RH}(\%)$ & Temperature $\left({ }^{\circ} \mathrm{C}\right)$ & Atm. P. (mbar) \\
1-HUM/TMP-BDST & 94 & 28.9 & P-IN-BDST & 862 \\
2-HUM/TMP-BDST & 91.6 & 28.9 & & \\
\hline
\end{tabular}


Single Patch q(film) Test Summary and Mass Balance - Rough Surface

Test: Patch $4,13.5 \mathrm{~cm}$ right of patch center $33^{\circ}$

\begin{tabular}{|c|c|c|c|c|}
\hline & Initial (g) & Final (g) & Difference $(\mathrm{g})$ & $\%$ of Input \\
\hline Water Input & $=-124.6$ & -270.8 & 14146.2 & $100 \%$ \\
\hline Evap & 86.9 & 86.2 & 0.7 & \\
\hline G1-1 & 64.3 & 149.2 & 84.9 & \multirow[b]{5}{*}{$64.4 \%$} \\
\hline G1-2 & 55.5 & 64.7 & 9.2 & \\
\hline G1-3 & & & 0 & \\
\hline G1-4 & & & 0 & \\
\hline SS-1 & & & 0 & \\
\hline G2-1 & & & 0 & \multirow[b]{5}{*}{$0.0 \%$} \\
\hline G2-2 & & & 0 & \\
\hline G2-3 & & & 0 & \\
\hline G2-4 & & & 0 & \\
\hline SS-2 & & & 0 & \\
\hline G3-1 & & & 0 & \multirow[b]{5}{*}{$0.0 \%$} \\
\hline G3-2 & & & 0 & \\
\hline G3-3 & & & 0 & \\
\hline G3-4 & & & 0 & \\
\hline SS-3 & & & 0 & \\
\hline G4-1 & & & 0 & \multirow[b]{5}{*}{$0.0 \%$} \\
\hline G4-2 & & & 0 & \\
\hline G4-3 & & & 0 & \\
\hline G4-4 & & & 0 & \\
\hline SS-4 & & & 0 & \\
\hline B1 & & & 0 & $0.0 \%$ \\
\hline B2 & 108.0 & 108.1 & 0.1 & $0.1 \%$ \\
\hline B3 & & & 0 & $0.0 \%$ \\
\hline B4 & 106.2 & 107.5 & 1.3 & $0.9 \%$ \\
\hline B5 & & & 0 & $0.0 \%$ \\
\hline B6 & & & 0 & $0.0 \%$ \\
\hline DS in-1 & 47.9 & 61.3 & 13.4 & \multirow[b]{2}{*}{$9.2 \%$} \\
\hline DS in-2 & & & 0 & \\
\hline DS out-1 & 49.3 & 53.9 & 4.6 & \multirow[b]{4}{*}{$3.1 \%$} \\
\hline DS out- 2 & & & 0 & \\
\hline DS out-3 & & & 0 & \\
\hline DS out-4 & & & 0 & \\
\hline Other & & & 0 & $0.0 \%$ \\
\hline
\end{tabular}

Test Date: $\quad 7 / 11 / 02$

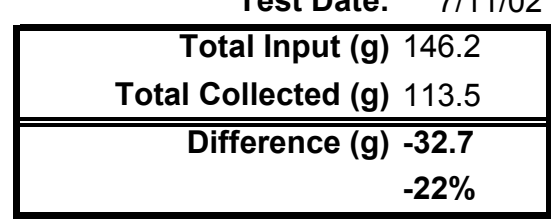

\begin{tabular}{|lr|}
\hline \multicolumn{2}{|c|}{ Evaporation } \\
Evap Pan Tare Wt (g) & 67.7 \\
Evaporation $(\mathrm{g})$ & 0.7 \\
\hline \% Evaporation & $\mathbf{3 . 6 5 \%}$ \\
\hline
\end{tabular}

\begin{tabular}{|lr|}
\hline \multicolumn{2}{|c|}{ Mass Flow Rate } \\
Test Duration (min) & 30.08 \\
Flow Rate (g/min) & 4.86 \\
\hline
\end{tabular}

\begin{tabular}{|lr|}
\hline \multicolumn{2}{|c|}{ Reference Towel Mass } \\
Initial Mass $(\mathrm{g})$ & 7.897 \\
Final Mass $(\mathrm{g})$ & 7.994 \\
\hline \hline Difference $(\mathrm{g})$ & 0.10 \\
\hline
\end{tabular}

\begin{tabular}{|lcccc|}
\hline \multicolumn{4}{l}{ Test Chamber Environment } \\
\hline Initial Conditions & $\mathrm{RH}(\%)$ & Temperature $\left({ }^{\circ} \mathrm{C}\right)$ & Atm. P. (mbar) \\
1-HUM/TMP-BDST & 90.3 & 21.6 & P-IN-BDST & 858 \\
2-HUM/TMP-BDST & 87 & 22.5 & & \\
\hline Final Conditions & $\mathrm{RH}(\%)$ & Temperature $\left({ }^{\circ} \mathrm{C}\right)$ & & Atm. P. (mbar) \\
1-HUM/TMP-BDST & 92.7 & 23.5 & P-IN-BDST & 848 \\
2-HUM/TMP-BDST & 92.8 & 23.3 & & \\
\hline
\end{tabular}


Single Patch q(film) Test Summary and Mass Balance - Rough Surface

Test: Patch 5, $13.5 \mathrm{~cm}$ left of patch center, $16.5^{\circ}$

Test Date: $\quad 7 / 8 / 02$

\begin{tabular}{|c|c|c|c|c|c|}
\hline & Initial (g) & Final (g) & Difference (g) & $\%$ of Input & Total Input (g) 340.9 \\
\hline Water Input & -1.3 & -342.2 & 340.9 & $100 \%$ & Total Collected (g) 285.1 \\
\hline Evap & 117.6 & 117.7 & -0.1 & \multirow[b]{6}{*}{$73.9 \%$} & \multirow{2}{*}{$\begin{array}{r}\text { Difference }(\mathrm{g}) \\
-55.8 \\
-16 \%\end{array}$} \\
\hline G1-1 & 71.3 & 313.9 & 242.6 & & \\
\hline G1-2 & 48.6 & 58.0 & 9.4 & & \\
\hline G1-3 & & & 0 & & Evaporation \\
\hline G1-4 & & & 0 & & \multirow{2}{*}{$\begin{array}{l}\text { Evap Pan Tare Wt }(\mathrm{g}) \\
\text { Evaporation }(\mathrm{g})\end{array}$} \\
\hline SS-1 & & & 0 & & \\
\hline G2-1 & & & 0 & & \multirow[t]{2}{*}{ \% Evaporation } \\
\hline G2-2 & & & 0 & & \\
\hline G2-3 & & & 0 & & \\
\hline
\end{tabular}

\begin{tabular}{|c|c|c|c|c|c|c|}
\hline$y<-3$ & & & 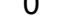 & & & \\
\hline G2-4 & & & 0 & & Mass Flo & \\
\hline SS-2 & & & 0 & $0.0 \%$ & Test Duration (min) & 65.20 \\
\hline G3-1 (SS) & 48.6 & 50.3 & 1.7 & & Flow Rate (g/min) & 5.23 \\
\hline G3-2 & & & 0 & & & \\
\hline
\end{tabular}

\begin{tabular}{|c|c|c|c|c|}
\hline G3-3 & & & 0 & \multirow[b]{3}{*}{$0.5 \%$} \\
\hline G3-4 & & & 0 & \\
\hline SS-3 & & & 0 & \\
\hline G4-1 & & & 0 & \multirow[b]{5}{*}{$0.0 \%$} \\
\hline G4-2 & & & 0 & \\
\hline G4-3 & & & 0 & \\
\hline G4-4 & & & 0 & \\
\hline SS-4 & & & 0 & \\
\hline$B 1$ & & & 0 & $0.0 \%$ \\
\hline $\mathrm{B} 2$ & 107.5 & 108.1 & 0.6 & $0.2 \%$ \\
\hline B3 & & & 0 & $0.0 \%$ \\
\hline B4 & & & 0 & $0.0 \%$ \\
\hline B5 & 109.2 & 111.7 & 2.5 & $0.7 \%$ \\
\hline $\mathrm{B} 6$ & 109.7 & 111.0 & 1.3 & $0.4 \%$ \\
\hline DS in-1 & & & 0 & \multirow[b]{2}{*}{$0.0 \%$} \\
\hline DS in-2 & & & 0 & \\
\hline DS out-1 & 56.3 & 83.3 & 27 & \multirow[b]{4}{*}{$7.9 \%$} \\
\hline DS out-2 & & & 0 & \\
\hline DS out-3 & & & 0 & \\
\hline DS out-4 & & & 0 & \\
\hline Other & & & 0 & $0.0 \%$ \\
\hline
\end{tabular}

\begin{tabular}{|lcccc|}
\hline \multicolumn{4}{ll}{ Test Chamber Environment } & \\
\hline Initial Conditions & $\mathrm{RH}(\%)$ & Temperature $\left({ }^{\circ} \mathrm{C}\right)$ & Atm. P. (mbar) \\
1-HUM/TMP-BDST & 92.3 & 26.3 & P-IN-BDST & 864 \\
2-HUM/TMP-BDST & 93.4 & 26.1 & & \\
\hline Final Conditions & $\mathrm{RH}(\%)$ & Temperature $\left({ }^{\circ} \mathrm{C}\right)$ & Atm. P. (mbar) \\
1-HUM/TMP-BDST & 95.7 & 28.3 & P-IN-BDST & 869 \\
2-HUM/TMP-BDST & 94.6 & 28.2 & & \\
\hline
\end{tabular}


Single Patch q(film) Test Summary and Mass Balance - Rough Surface

Test: Patch 5, $13.5 \mathrm{~cm}$ left of patch center, crown

Test Date: $\quad 7 / 10 / 02$

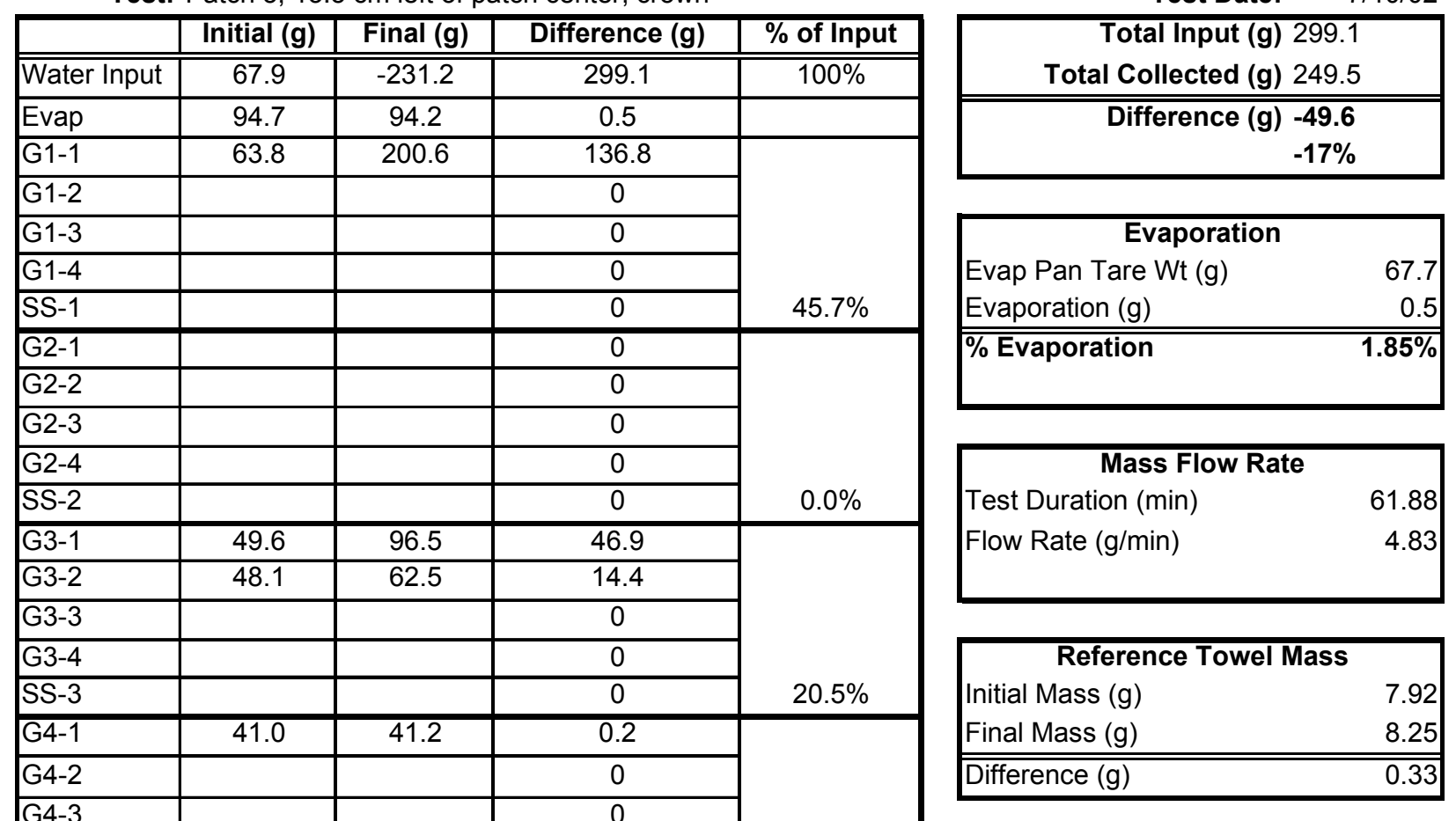

\begin{tabular}{|c|c|c|c|c|}
\hline G4-3 & & & 0 & \multirow[b]{3}{*}{$0.1 \%$} \\
\hline G4-4 & & & 0 & \\
\hline SS-4 & & & 0 & \\
\hline $\mathrm{B} 1$ & & & 0 & $0.0 \%$ \\
\hline $\mathrm{B} 2$ & 108.3 & 108.4 & 0.1 & $0.0 \%$ \\
\hline B3 & & & 0 & $0.0 \%$ \\
\hline B4 & & & 0 & $0.0 \%$ \\
\hline B5 & 109.5 & 115.4 & 5.9 & $2.0 \%$ \\
\hline B6 & 110.6 & 112.3 & 1.7 & $0.6 \%$ \\
\hline DS in-1 & & & 0 & \multirow[b]{2}{*}{$0.0 \%$} \\
\hline DS in-2 & & & 0 & \\
\hline DS out-1 & 65.5 & 109.0 & 43.5 & \multirow[b]{4}{*}{$14.5 \%$} \\
\hline DS out- 2 & & & 0 & \\
\hline DS out-3 & & & 0 & \\
\hline DS out-4 & & & 0 & \\
\hline Other & & & 0 & $0.0 \%$ \\
\hline
\end{tabular}

\begin{tabular}{|c|c|c|c|c|}
\hline \multicolumn{5}{|c|}{ Test Chamber Environment } \\
\hline Initial Conditions & $\mathrm{RH}(\%)$ & Temperature $\left({ }^{\circ} \mathrm{C}\right)$ & & Atm. P. (mbar) \\
\hline 1-HUM/TMP-BDST & 90.4 & 24.7 & P-IN-BDST & 856 \\
\hline 2-HUM/TMP-BDST & 88.9 & 24.9 & & \\
\hline Final Conditions & $\mathrm{RH}(\%)$ & Temperature $\left({ }^{\circ} \mathrm{C}\right)$ & & Atm. P. (mbar) \\
\hline 1-HUM/TMP-BDST & 94.1 & 26.6 & P-IN-BDST & 846 \\
\hline 2-HUM/TMP-BDST & 90.1 & 26.9 & & \\
\hline
\end{tabular}


Single Patch q(film) Test Summary and Mass Balance - Rough Surface

Test: Patch 5, centerline, crown

\begin{tabular}{|c|c|c|c|c|}
\hline & Initial & Final & Difference & $\%$ of Input \\
\hline Water Input & $4 \quad 42.2$ & $=245.5$ & 287.7 & $100 \%$ \\
\hline Evap & 92.2 & 91.8 & 0.4 & \\
\hline$\overline{G 1-1}$ & 49.6 & 66.3 & 16.7 & \multirow[b]{5}{*}{$5.8 \%$} \\
\hline G1-2 & & & 0 & \\
\hline$\overline{\mathrm{G} 1-3}$ & & & 0 & \\
\hline $\mathrm{G} 1-4$ & & & 0 & \\
\hline SS-1 & & & 0 & \\
\hline $\bar{G} 2-1$ & & & 0 & \multirow[b]{5}{*}{$0.0 \%$} \\
\hline G2-2 & & & 0 & \\
\hline G2-3 & & & 0 & \\
\hline$\overline{\mathrm{G} 2-4}$ & & & 0 & \\
\hline SS-2 & & & 0 & \\
\hline$\overline{\text { G3-1 }}$ & 48.3 & 111.9 & 63.6 & \multirow[b]{5}{*}{$28.8 \%$} \\
\hline G3-2 & 48.4 & 67.6 & 19.2 & \\
\hline G3-3 & & & 0 & \\
\hline G3-4 & & & 0 & \\
\hline SS-3 & & & 0 & \\
\hline G4-1 & 40.9 & 42.0 & 1.1 & \multirow[b]{5}{*}{$0.4 \%$} \\
\hline G4-2 & & & 0 & \\
\hline G4-3 & & & 0 & \\
\hline G4-4 & & & 0 & \\
\hline SS-4 & & & 0 & \\
\hline $\mathrm{B} 1$ & & & 0 & $0.0 \%$ \\
\hline$B 2$ & 107.7 & 108.3 & 0.6 & $0.2 \%$ \\
\hline B3 & 105.9 & 106.5 & 0.6 & $0.2 \%$ \\
\hline $\mathrm{B} 4$ & & & 0 & $0.0 \%$ \\
\hline $\mathrm{B} 5$ & & & 0 & $0.0 \%$ \\
\hline B6 & 110.3 & 111.4 & 1.1 & $0.4 \%$ \\
\hline$\overline{D S}$ in-1 & & & 0 & \multirow[b]{2}{*}{$0.0 \%$} \\
\hline DS in-2 & & & 0 & \\
\hline DS out-1 & 63.3 & 117.7 & 54.4 & \multirow[b]{4}{*}{$18.9 \%$} \\
\hline DS out-2 & & & 0 & \\
\hline DS out-3 & & & 0 & \\
\hline DS out-4 & & & 0 & \\
\hline Other & & & 0 & $0.0 \%$ \\
\hline
\end{tabular}

Test Date: $\quad 7 / 10 / 02$
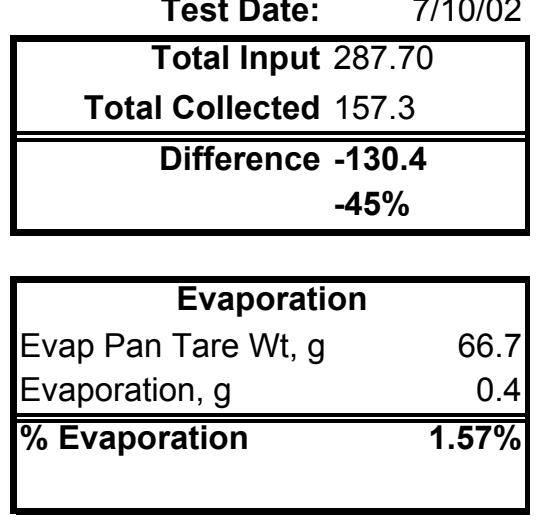

\begin{tabular}{|lr|}
\hline \multicolumn{2}{|c|}{ Mass Flow Rate } \\
Test Duration, min & 60 \\
Flow Rate, g/min & 4.80 \\
& \\
\hline
\end{tabular}

\begin{tabular}{|lr|}
\hline \multicolumn{2}{|c|}{ Reference Towel Mass } \\
Initial Mass $(\mathrm{g})$ & 7.568 \\
Final Mass $(\mathrm{g})$ & 8.22 \\
\hline \hline Difference $(\mathrm{g})$ & 0.65 \\
\hline
\end{tabular}

\begin{tabular}{|lcccc|}
\hline \multicolumn{4}{l}{ Test Chamber Environment } & \multicolumn{3}{l|}{} \\
\hline Initial Conditions & $\mathrm{RH}(\%)$ & Temperature $\left({ }^{\circ} \mathrm{C}\right)$ & & Atm. P. (mbar) \\
1-HUM/TMP-BDST & 83 & 24 & P-IN-BDST & 855 \\
2-HUM/TMP-BDST & 83.2 & 24 & & \\
\hline Final Conditions & $\mathrm{RH}(\%)$ & Temperature $\left({ }^{\circ} \mathrm{C}\right)$ & & Atm. P. (mbar) \\
1-HUM/TMP-BDST & 94.2 & 26.9 & P-IN-BDST & 854 \\
2-HUM/TMP-BDST & 92.3 & 26.9 & & \\
\hline
\end{tabular}


Single Patch q(film) Test Summary and Mass Balance - Rough Surface

Test: Patch 5, $13.5 \mathrm{~cm}$ right of patch center, $33^{\circ}$

\begin{tabular}{|c|c|c|c|c|}
\hline & Initial & Final & Difference & $\%$ of Input \\
\hline Water Input & "58.4 & -108.2 & 166.6 & $100 \%$ \\
\hline Evap & 87.8 & 87.3 & 0.5 & \\
\hline$\overline{G 1-1}$ & 63.7 & 179.1 & 115.4 & \multirow[b]{5}{*}{$76.2 \%$} \\
\hline $\mathrm{G} 1-2$ & 55.4 & 67.0 & 11.6 & \\
\hline $\mathrm{G} 1-3$ & & & 0 & \\
\hline G1-4 & & & 0 & \\
\hline SS-1 & & & 0 & \\
\hline$\overline{\mathrm{G} 2-1}$ & & & 0 & \multirow[b]{5}{*}{$0.0 \%$} \\
\hline G2-2 & & & 0 & \\
\hline G2-3 & & & 0 & \\
\hline $\bar{G} 2-4$ & & & 0 & \\
\hline SS-2 & & & 0 & \\
\hline G3-1 & & & 0 & \multirow[b]{5}{*}{$0.0 \%$} \\
\hline G3-2 & & & 0 & \\
\hline G3-3 & & & 0 & \\
\hline G3-4 & & & 0 & \\
\hline SS-3 & & & 0 & \\
\hline G4-1 & & & 0 & \multirow[b]{5}{*}{$0.0 \%$} \\
\hline G4-2 & & & 0 & \\
\hline G4-3 & & & 0 & \\
\hline G4-4 & & & $\overline{0}$ & \\
\hline SS-4 & & & 0 & \\
\hline B1 & & & 0 & $0.0 \%$ \\
\hline B2 & 108.0 & 108.0 & 0 & $0.0 \%$ \\
\hline B3 & & & 0 & $0.0 \%$ \\
\hline B4 & & & 0 & $0.0 \%$ \\
\hline B5 & 112.2 & 113.8 & 1.6 & $1.0 \%$ \\
\hline B6 & & & 0 & $0.0 \%$ \\
\hline DS in-1 & 41.2 & 41.8 & 0.6 & \multirow[b]{2}{*}{$0.4 \%$} \\
\hline DS in-2 & & & 0 & \\
\hline DS out-1 & 63.7 & 68.6 & 4.9 & \multirow[b]{4}{*}{$2.9 \%$} \\
\hline DS out- 2 & & & 0 & \\
\hline DS out-3 & & & 0 & \\
\hline DS out-4 & & & 0 & \\
\hline Other & & & 0 & $0.0 \%$ \\
\hline
\end{tabular}

Test Date: $\quad 7 / 11 / 02$

Total Input 166.6

Total Collected 134.1

Difference $\mathbf{- 3 2 . 5}$ $-20 \%$

Evaporation

Evap Pan Tare Wt, g $\quad 66.7$

Evaporation, g

0.5

\begin{tabular}{lr}
\hline \% Evaporation $\quad 2.37 \%$ \\
\hline
\end{tabular}

\begin{tabular}{|lr|}
\hline \multicolumn{2}{|c|}{ Mass Flow Rate } \\
Test Duration, min & 34.03 \\
Flow Rate, g/min & 4.90 \\
& \\
\hline
\end{tabular}

\begin{tabular}{|lr|}
\hline \multicolumn{2}{|c|}{ Reference Towel Mass } \\
Initial Mass $(\mathrm{g})$ & 7.956 \\
Final Mass $(\mathrm{g})$ & 7.97 \\
\hline \hline Difference $(\mathrm{g})$ & 0.01 \\
\hline
\end{tabular}

Test Chamber Environment

\begin{tabular}{|lcccc}
\hline Initial Conditions & $\mathrm{RH}(\%)$ & Temperature $\left({ }^{\circ} \mathrm{C}\right)$ & & Atm. P. (mbar) \\
1-HUM/TMP-BDST & 91.5 & 21.3 & P-IN-BDST & 848 \\
2-HUM/TMP-BDST & 93.9 & 21 & & \\
\hline Final Conditions & $\mathrm{RH}(\%)$ & Temperature $\left({ }^{\circ} \mathrm{C}\right)$ & & Atm. P. (mbar) \\
1-HUM/TMP-BDST & 92.9 & 22.4 & P-IN-BDST & 859 \\
2-HUM/TMP-BDST & 92.9 & 22.3 & &
\end{tabular}


Single Patch q(film) Test Summary and Mass Balance - Rough Surface

Test: Patch 5, Centerline, 16.5

\begin{tabular}{|c|c|c|c|c|}
\hline & Initial & Final & Difference & $\%$ of Input \\
\hline Water Input & 739.2 & 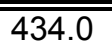 & 305.2 & $100 \%$ \\
\hline Evap & 100.5 & 100.3 & 0.2 & \\
\hline $\mathrm{G} 1-1$ & 50.1 & 60.5 & 10.4 & \multirow[b]{5}{*}{$3.4 \%$} \\
\hline G1-2 & & & 0 & \\
\hline $\mathrm{G} 1-3$ & & & 0 & \\
\hline G1-4 & & & 0 & \\
\hline SS-1 & & & 0 & \\
\hline $\bar{G} 2-1$ & & & 0 & \multirow[b]{5}{*}{$0.0 \%$} \\
\hline G2-2 & & & 0 & \\
\hline G2-3 & & & 0 & \\
\hline G2-4 & & & 0 & \\
\hline SS-2 & & & 0 & \\
\hline$\overline{\mathrm{G} 3-1}$ & 41.2 & $\overline{41.4}$ & 0.2 & \multirow[b]{5}{*}{$0.1 \%$} \\
\hline G3-2 & & & 0 & \\
\hline G3-3 & & & 0 & \\
\hline G3-4 & & & 0 & \\
\hline SS-3 & & & 0 & \\
\hline G4-1 & 42.1 & 42.6 & 0.5 & \multirow[b]{5}{*}{$0.2 \%$} \\
\hline G4-2 & & & 0 & \\
\hline G4-3 & & & 0 & \\
\hline G4-4 & & & 0 & \\
\hline SS-4 & & & $\overline{0}$ & \\
\hline$\overline{B 1}$ & & & $\overline{0}$ & $0.0 \%$ \\
\hline B2 & & & 0 & $0.0 \%$ \\
\hline B3 & & & 0 & $0.0 \%$ \\
\hline B4 & & & 0 & $0.0 \%$ \\
\hline B5 & 109.4 & 320.1 & 210.7 & $69.0 \%$ \\
\hline$\overline{B 6}$ & & & 0 & $0.0 \%$ \\
\hline$\overline{D S}$ in-1 & & & 0 & \multirow[b]{2}{*}{$0.0 \%$} \\
\hline$\overline{D S}$ in-2 & & & 0 & \\
\hline DS out-1 & 63.9 & 101.6 & 37.7 & \multirow[b]{4}{*}{$12.4 \%$} \\
\hline DS out- 2 & & & 0 & \\
\hline DS out-3 & & & 0 & \\
\hline DS out-4 & & & 0 & \\
\hline Other & & & 0 & $0.0 \%$ \\
\hline
\end{tabular}

Test Date: $\quad 7 / 3 / 02$

Total Input 305.2

Total Collected 259.5

Difference -45.7

$-15 \%$

Evaporation

Evap Pan Tare Wt, g $\quad 66.7$

Evaporation, $g$

0.2

\% Evaporation $\quad 0.59 \%$

Mass Flow Rate

Test Duration, min

Flow Rate, $\mathrm{g} / \mathrm{min}$

Reference Towel Mass

Initial Mass (g) $\quad 7.595$

Final Mass $(\mathrm{g}) \quad 7.858$

\begin{tabular}{ll}
\hline Difference $(\mathrm{g})$ & 0.26 \\
\hline
\end{tabular}

Test Chamber Environment

\begin{tabular}{|lcccc|}
\hline Initial Conditions & $\mathrm{RH}(\%)$ & Temperature $\left({ }^{\circ} \mathrm{C}\right)$ & Atm. P. (mbar) \\
1-HUM/TMP-BDST & 91.1 & 27 & P-IN-BDST & 863 \\
2-HUM/TMP-BDST & 88.9 & 26.9 & & \\
\hline Final Conditions & $\mathrm{RH}(\%)$ & Temperature $\left({ }^{\circ} \mathrm{C}\right)$ & Atm. P. (mbar) \\
1-HUM/TMP-BDST & 94.3 & 28.6 & P-IN-BDST & 864 \\
2-HUM/TMP-BDST & 94.4 & 28.2 & & \\
\hline
\end{tabular}


Multiple Patch Test Summary and Mass Balance - Rough Surface

Test: $81 \mathrm{~cm}$ left of DS center, crown

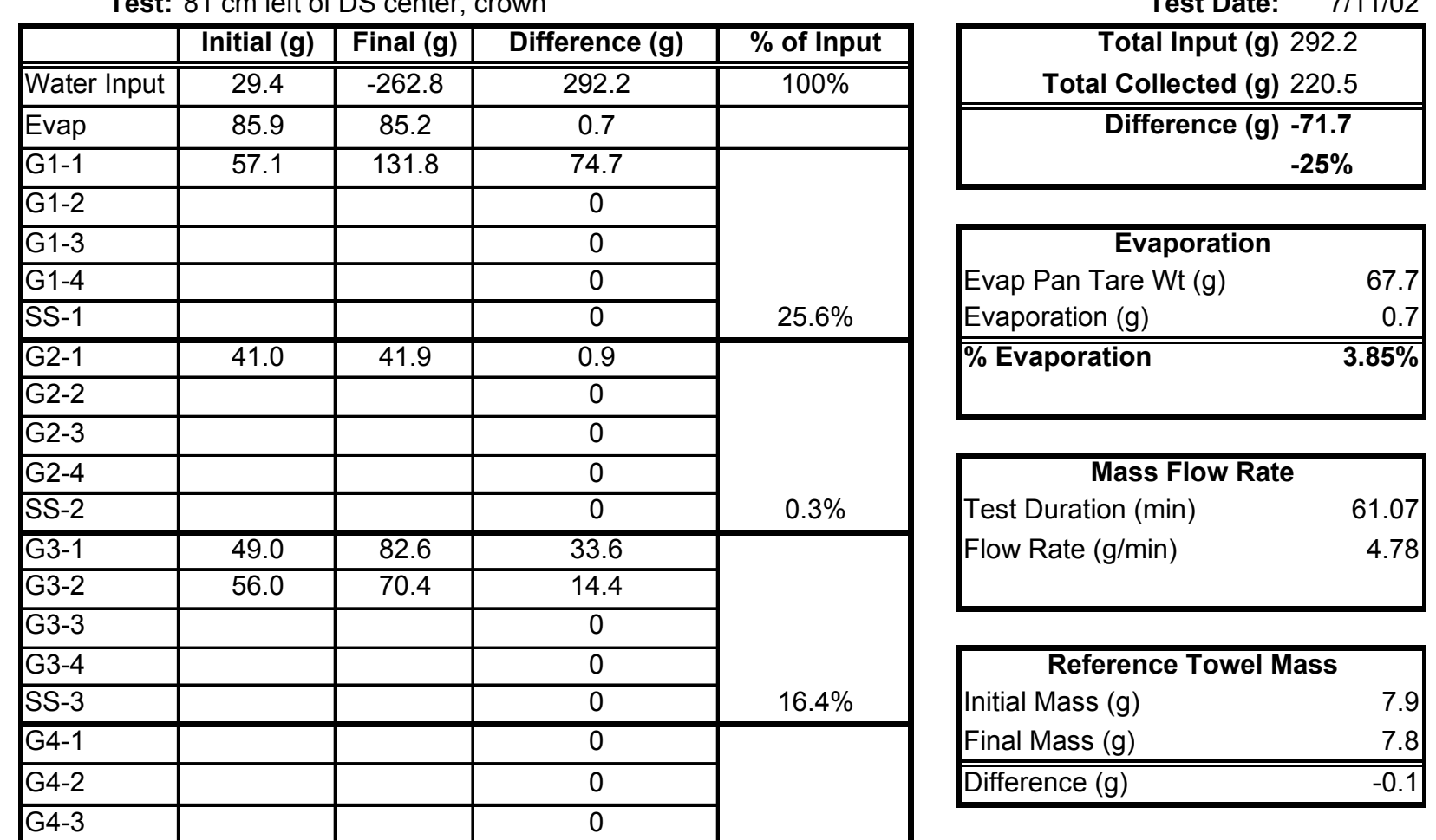

\begin{tabular}{|c|c|c|c|c|}
\hline & & & & \\
\hline G4-4 & & & 0 & \\
\hline SS-4 & & & 0 & $0.0 \%$ \\
\hline $\mathrm{B} 1$ & 106.2 & 164.8 & 58.6 & $20.1 \%$ \\
\hline B2 & & & 0 & $0.0 \%$ \\
\hline B3 & & & 0 & $0.0 \%$ \\
\hline B4 & 106.4 & 106.7 & 0.3 & $0.1 \%$ \\
\hline B5 & & & 0 & $0.0 \%$ \\
\hline B6 & & & 0 & $0.0 \%$ \\
\hline DS in-1 & & & 0 & \\
\hline DS in-2 & & & 0 & $0.0 \%$ \\
\hline DS out-1 & 63.9 & 101.9 & 38 & \\
\hline DS out- 2 & & & 0 & \\
\hline DS out-3 & & & 0 & \\
\hline DS out-4 & & & 0 & $13.0 \%$ \\
\hline Other & & & 0 & $0.0 \%$ \\
\hline
\end{tabular}

\begin{tabular}{|lcccc|}
\hline \multicolumn{4}{l}{ Test Chamber Environment } \\
\hline Initial Conditions & $\mathrm{RH}(\%)$ & Temperature $\left({ }^{\circ} \mathrm{C}\right)$ & Atm. P. (mbar) \\
1-HUM/TMP-BDST & 92.6 & 23.9 & $\mathrm{P}-\mathrm{IN}-\mathrm{BDST}$ & 856 \\
2-HUM/TMP-BDST & 92.3 & 24 & & \\
\hline Final Conditions & $\mathrm{RH}(\%)$ & Temperature $\left({ }^{\circ} \mathrm{C}\right)$ & Atm. P. (mbar) \\
1-HUM/TMP-BDST & 91.4 & 24.4 & P-IN-BDST & 855 \\
2-HUM/TMP-BDST & 89.6 & 24.4 & & \\
\hline
\end{tabular}


Multiple Patch Test Summary and Mass Balance - Rough Surface

Test: $27 \mathrm{~cm}$ left of DS center, crown

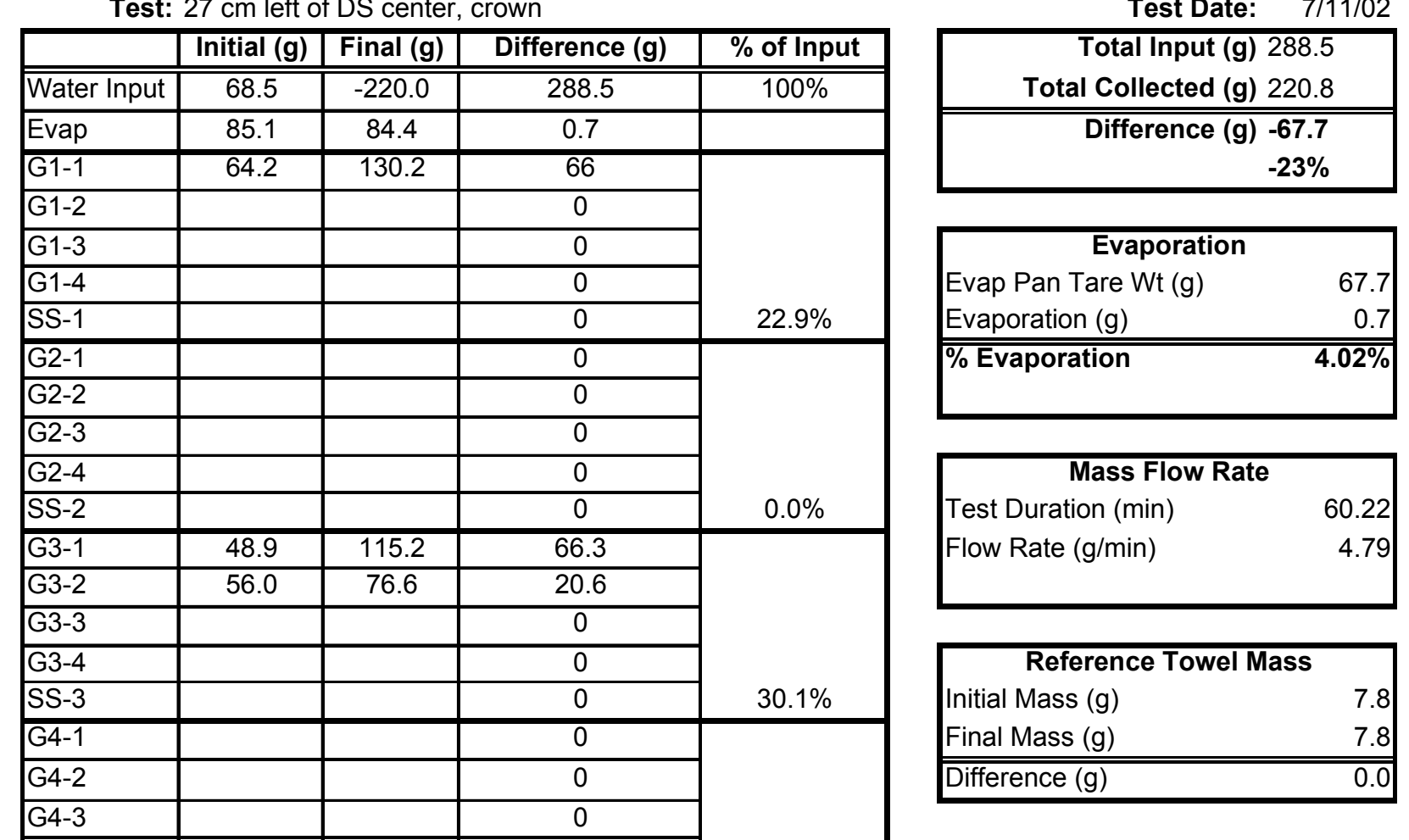

\begin{tabular}{|c|c|c|c|c|}
\hline & & & & \multirow[b]{3}{*}{$0.0 \%$} \\
\hline G4-4 & & & 0 & \\
\hline SS-4 & & & 0 & \\
\hline B1 & & & 0 & $0.0 \%$ \\
\hline B2 & 107.9 & 112.2 & 4.3 & $1.5 \%$ \\
\hline B3 & & & 0 & $0.0 \%$ \\
\hline B4 & 106.6 & 111.9 & 5.3 & $1.8 \%$ \\
\hline B5 & & & 0 & $0.0 \%$ \\
\hline B6 & 110.1 & 113.5 & 3.4 & $1.2 \%$ \\
\hline DS in-1 & 41.0 & 45.1 & 4.1 & \multirow[b]{2}{*}{$1.4 \%$} \\
\hline DS in-2 & & & 0 & \\
\hline DS out-1 & 64.2 & 115.0 & 50.8 & \multirow[b]{4}{*}{$17.6 \%$} \\
\hline DS out-2 & & & 0 & \\
\hline DS out-3 & & & 0 & \\
\hline DS out-4 & & & 0 & \\
\hline Other & & & 0 & $0.0 \%$ \\
\hline
\end{tabular}

\begin{tabular}{|lcccc|}
\hline \multicolumn{4}{l}{ Test Chamber Environment } \\
\hline Initial Conditions & $\mathrm{RH}(\%)$ & Temperature $\left({ }^{\circ} \mathrm{C}\right)$ & Atm. P. (mbar) \\
1-HUM/TMP-BDST & 89.3 & 24.2 & P-IN-BDST & 854 \\
2-HUM/TMP-BDST & 86.8 & 24.6 & & \\
\hline Final Conditions & $\mathrm{RH}(\%)$ & Temperature $\left({ }^{\circ} \mathrm{C}\right)$ & & Atm. P. (mbar) \\
1-HUM/TMP-BDST & 90.6 & 24.9 & P-IN-BDST & 848 \\
2-HUM/TMP-BDST & 86.3 & 25.5 & & \\
\hline
\end{tabular}


Multiple Patch Test Summary and Mass Balance - Rough Surface

Test: $27 \mathrm{~cm}$ right of DS center, crown

Test Date: $\quad 7 / 12 / 02$

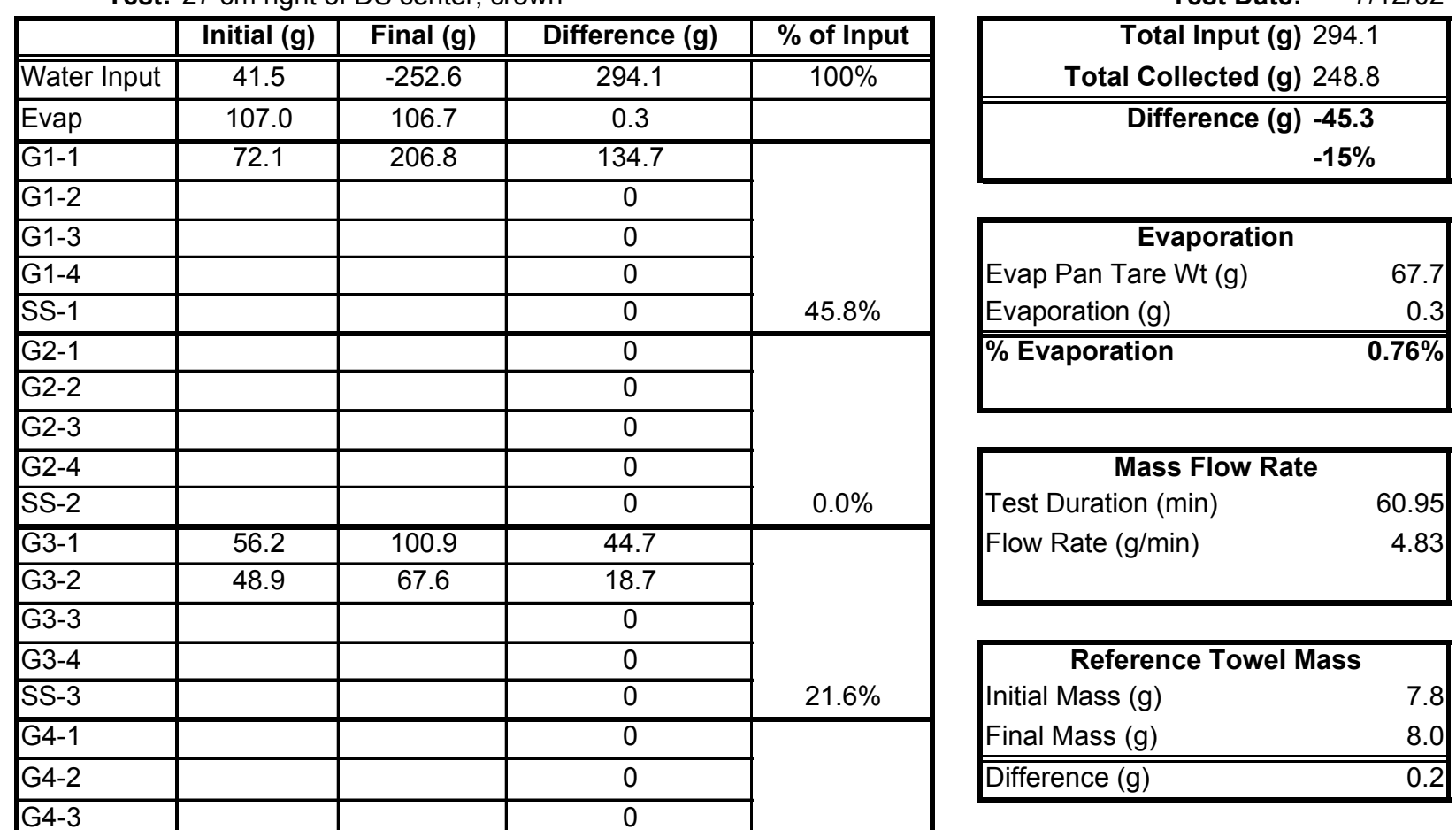

\begin{tabular}{|c|c|c|c|c|}
\hline G4-4 & & & 0 & \multirow[b]{2}{*}{$0.0 \%$} \\
\hline SS-4 & & & 0 & \\
\hline $\mathrm{B} 1$ & & & 0 & $0.0 \%$ \\
\hline B2 & 108.0 & 108.3 & 0.3 & $0.1 \%$ \\
\hline B3 & & & 0 & $0.0 \%$ \\
\hline B4 & & & 0 & $0.0 \%$ \\
\hline B5 & 109.5 & 109.7 & 0.2 & $0.1 \%$ \\
\hline B6 & 110.1 & 113.3 & 3.2 & $1.1 \%$ \\
\hline DS in-1 & & & 0 & \multirow[b]{2}{*}{$0.0 \%$} \\
\hline DS in-2 & & & 0 & \\
\hline DS out-1 & 63.6 & 110.6 & 47 & \multirow[b]{4}{*}{$16.0 \%$} \\
\hline DS out-2 & & & 0 & \\
\hline DS out-3 & & & 0 & \\
\hline DS out-4 & & & 0 & \\
\hline Other & & & 0 & $0.0 \%$ \\
\hline
\end{tabular}

\begin{tabular}{|lcccc|}
\hline \multicolumn{4}{l}{ Test Chamber Environment } & \multicolumn{4}{l|}{} \\
\hline Initial Conditions & $\mathrm{RH}(\%)$ & Temperature $\left({ }^{\circ} \mathrm{C}\right)$ & Atm. P. (mbar) \\
1-HUM/TMP-BDST & 88.9 & 19.8 & P-IN-BDST & 861 \\
2-HUM/TMP-BDST & 92 & 19.6 & & \\
\hline Final Conditions & $\mathrm{RH}(\%)$ & Temperature $\left({ }^{\circ} \mathrm{C}\right)$ & Atm. P. (mbar) \\
1-HUM/TMP-BDST & 92.4 & 21.4 & P-IN-BDST & 858 \\
2-HUM/TMP-BDST & 89.9 & 22.7 & & \\
\hline
\end{tabular}


Multiple Patch Test Summary and Mass Balance - Rough Surface

Test: $81 \mathrm{~cm}$ right of DS center, crown

Test Date: $\quad 7 / 12 / 02$

\begin{tabular}{|c|c|c|c|c|c|}
\hline & Initial (g) & Final (g) & Difference (g) & $\%$ of Input & \multirow{2}{*}{$\begin{aligned} \text { Total Input }(\mathbf{g}) & 290.0 \\
\text { Total Collected }(\mathrm{g}) & 251.6\end{aligned}$} \\
\hline Water Input & 78.7 & -211.3 & 290 & $100 \%$ & \\
\hline Evap & 106.3 & 105.5 & 0.8 & & \multirow{2}{*}{$\begin{array}{r}\text { Difference (g) }-38.4 \\
-13 \%\end{array}$} \\
\hline G1-1 & 71.8 & 220.8 & 149 & \multirow[b]{5}{*}{$51.4 \%$} & \\
\hline G1-2 & & & 0 & & \\
\hline G1-3 & & & 0 & & Evaporation \\
\hline G1-4 & & & 0 & & Evap Pan Tare Wt (g) \\
\hline SS-1 & & & 0 & & Evaporation (g) $\quad 0.8$ \\
\hline G2-1 & & & 0 & & \% Evaporation $\quad 2.07 \%$ \\
\hline G2-2 & & & 0 & & \\
\hline G2-3 & & & 0 & & \\
\hline G2-4 & & & 0 & & Mass Flow Rate \\
\hline SS-2 & & & 0 & $0.0 \%$ & Test Duration (min) \\
\hline G3-1 & 56.2 & 98.2 & 42 & & Flow Rate (g/min) \\
\hline G3-2 & 56.5 & 71.4 & 14.9 & & \\
\hline G3-3 & & & 0 & & \\
\hline G3-4 & & & 0 & & Reference Towel Mass \\
\hline SS-3 & & & 0 & $19.6 \%$ & Initial Mass $(\mathrm{g})$ \\
\hline G4-1 & & & 0 & & Final Mass $(\mathrm{g})$ \\
\hline G4-2 & & & 0 & & Difference (g) \\
\hline G4-3 & & & 0 & & \\
\hline
\end{tabular}

\begin{tabular}{|c|c|c|c|c|}
\hline G4-4 & & & 0 & \multirow[b]{2}{*}{$2.0 \%$} \\
\hline SS-4 & 41.0 & 46.7 & 5.7 & \\
\hline B1 & & & 0 & $0.0 \%$ \\
\hline B2 & & & 0 & $0.0 \%$ \\
\hline B3 & 107.0 & 107.2 & 0.2 & $0.1 \%$ \\
\hline B4 & & & 0 & $0.0 \%$ \\
\hline B5 & 109.6 & 110.6 & 1 & $0.3 \%$ \\
\hline B6 & 110.3 & 110.2 & -0.1 & $0.0 \%$ \\
\hline DS in-1 & & & 0 & \multirow[b]{2}{*}{$0.0 \%$} \\
\hline DS in-2 & & & 0 & \\
\hline DS out-1 & 64.3 & 103.2 & 38.9 & \multirow[b]{4}{*}{$13.4 \%$} \\
\hline DS out- 2 & & & 0 & \\
\hline DS out-3 & & & 0 & \\
\hline DS out-4 & & & 0 & \\
\hline Other & & & 0 & $0.0 \%$ \\
\hline
\end{tabular}

\begin{tabular}{|lcccc|}
\hline \multicolumn{4}{l}{ Test Chamber Environment } \\
\hline Initial Conditions & $\mathrm{RH}(\%)$ & Temperature $\left({ }^{\circ} \mathrm{C}\right)$ & Atm. P. (mbar) \\
1-HUM/TMP-BDST & 92.3 & 22.3 & P-IN-BDST & 863 \\
2-HUM/TMP-BDST & 88.5 & 22.8 & & \\
\hline Final Conditions & $\mathrm{RH}(\%)$ & Temperature $\left({ }^{\circ} \mathrm{C}\right)$ & Atm. P. (mbar) \\
1-HUM/TMP-BDST & 92.3 & 24.1 & P-IN-BDST & 858 \\
2-HUM/TMP-BDST & 89.5 & 24.4 & & \\
\hline
\end{tabular}


Multiple Patch Test Summary and Mass Balance - Rough Surface

Test: $81 \mathrm{~cm}$ right of DS center, $16.5^{\circ}$

\begin{tabular}{|c|c|c|c|c|c|c|}
\hline & Initial (g) & Final (g) & Difference $(\mathrm{g})$ & $\%$ of Input & \multirow{2}{*}{\multicolumn{2}{|c|}{ Total Collected (g) 230.7}} \\
\hline Water Input & 16.9 & -265.9 & 282.8 & \multirow[t]{2}{*}{$100 \%$} & & \\
\hline Evap & 105.3 & 104.7 & 0.6 & & \multirow{2}{*}{\multicolumn{2}{|c|}{ 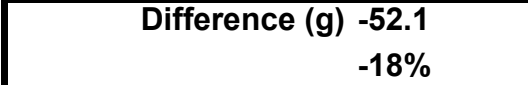 }} \\
\hline G1-1 & 64.9 & 257.3 & 192.4 & \multirow[b]{5}{*}{$69.7 \%$} & & \\
\hline G1-2 & 41.1 & 45.9 & 4.8 & & & \\
\hline G1-3 & & & 0 & & \multicolumn{2}{|c|}{ Evaporation } \\
\hline G1-4 & & & 0 & & \multirow{2}{*}{\multicolumn{2}{|c|}{\begin{tabular}{|rr} 
Evap Pan Tare Wt $(\mathrm{g})$ & 67.7 \\
Evaporation $(\mathrm{g})$ & 0.6 \\
\end{tabular}}} \\
\hline SS-1 & & & 0 & & & \\
\hline G2-1 & & & 0 & \multirow[b]{5}{*}{$0.0 \%$} & \multirow[t]{2}{*}{$\%$ Evaporation } & \multirow[t]{2}{*}{$1.60 \%$} \\
\hline G2-2 & & & 0 & & & \\
\hline G2-3 & & & 0 & & & \\
\hline G2-4 & & & 0 & & \multicolumn{2}{|c|}{ Mass Flow Rate } \\
\hline SS-2 & & & 0 & & \multirow{3}{*}{$\begin{array}{l}\text { Test Duration }(\min ) \\
\text { Flow Rate }(\mathrm{g} / \mathrm{min})\end{array}$} & \multirow{3}{*}{$\begin{array}{r}60.03 \\
4.71\end{array}$} \\
\hline G3-1(SS) & 41.6 & 42.2 & 0.6 & & & \\
\hline G3-2 & & & 0 & & & \\
\hline G3-3 & & & 0 & & & \\
\hline
\end{tabular}

\begin{tabular}{|c|c|c|c|c|}
\hline G3-3 & & & 0 & \multirow[b]{3}{*}{$0.2 \%$} \\
\hline G3-4 & & & 0 & \\
\hline SS-3 & & & 0 & \\
\hline G4-1(SS) & 41.2 & 45.6 & 4.4 & \multirow[b]{5}{*}{$1.6 \%$} \\
\hline G4-2 & & & 0 & \\
\hline G4-3 & & & 0 & \\
\hline G4-4 & & & 0 & \\
\hline SS-4 & & & 0 & \\
\hline B1 & & & 0 & $0.0 \%$ \\
\hline B2 & & & 0 & $0.0 \%$ \\
\hline B3 & 108.1 & 108.9 & 0.8 & $0.3 \%$ \\
\hline B4 & & & 0 & $0.0 \%$ \\
\hline B5 & 109.4 & 110.2 & 0.8 & $0.3 \%$ \\
\hline B6 & 110.1 & 110.3 & 0.2 & $0.1 \%$ \\
\hline DS in-1 & & & 0 & \multirow[b]{2}{*}{$0.0 \%$} \\
\hline DS in-2 & & & 0 & \\
\hline DS out-1 & 64.3 & 91.0 & 26.7 & \multirow[b]{4}{*}{$9.4 \%$} \\
\hline DS out- 2 & & & 0 & \\
\hline DS out-3 & & & 0 & \\
\hline DS out-4 & & & 0 & \\
\hline Other & & & 0 & $0.0 \%$ \\
\hline
\end{tabular}

\begin{tabular}{|lr|}
\hline \multicolumn{2}{|c|}{ Reference Towel Mass } \\
Initial Mass $(\mathrm{g})$ & 7.9 \\
Final Mass $(\mathrm{g})$ & 8.1 \\
\hline \hline Difference $(\mathrm{g})$ & 0.2 \\
\hline
\end{tabular}

\begin{tabular}{|lcccc|}
\hline \multicolumn{5}{l}{ Test Chamber Environment } \\
\hline Initial Conditions & $\mathrm{RH}(\%)$ & Temperature $\left({ }^{\circ} \mathrm{C}\right)$ & Atm. P. (mbar) \\
1-HUM/TMP-BDST & 89 & 24.9 & P-IN-BDST & 858 \\
2-HUM/TMP-BDST & 89.2 & 24.7 & & \\
\hline Final Conditions & $\mathrm{RH}(\%)$ & Temperature $\left({ }^{\circ} \mathrm{C}\right)$ & Atm. P. (mbar) \\
1-HUM/TMP-BDST & 93.9 & 26.9 & P-IN-BDST & 867 \\
2-HUM/TMP-BDST & 93.1 & 26.7 & & \\
\hline
\end{tabular}


Multiple Patch Test Summary and Mass Balance - Rough Surface

Test: $27 \mathrm{~cm}$ right of DS center, 16.5

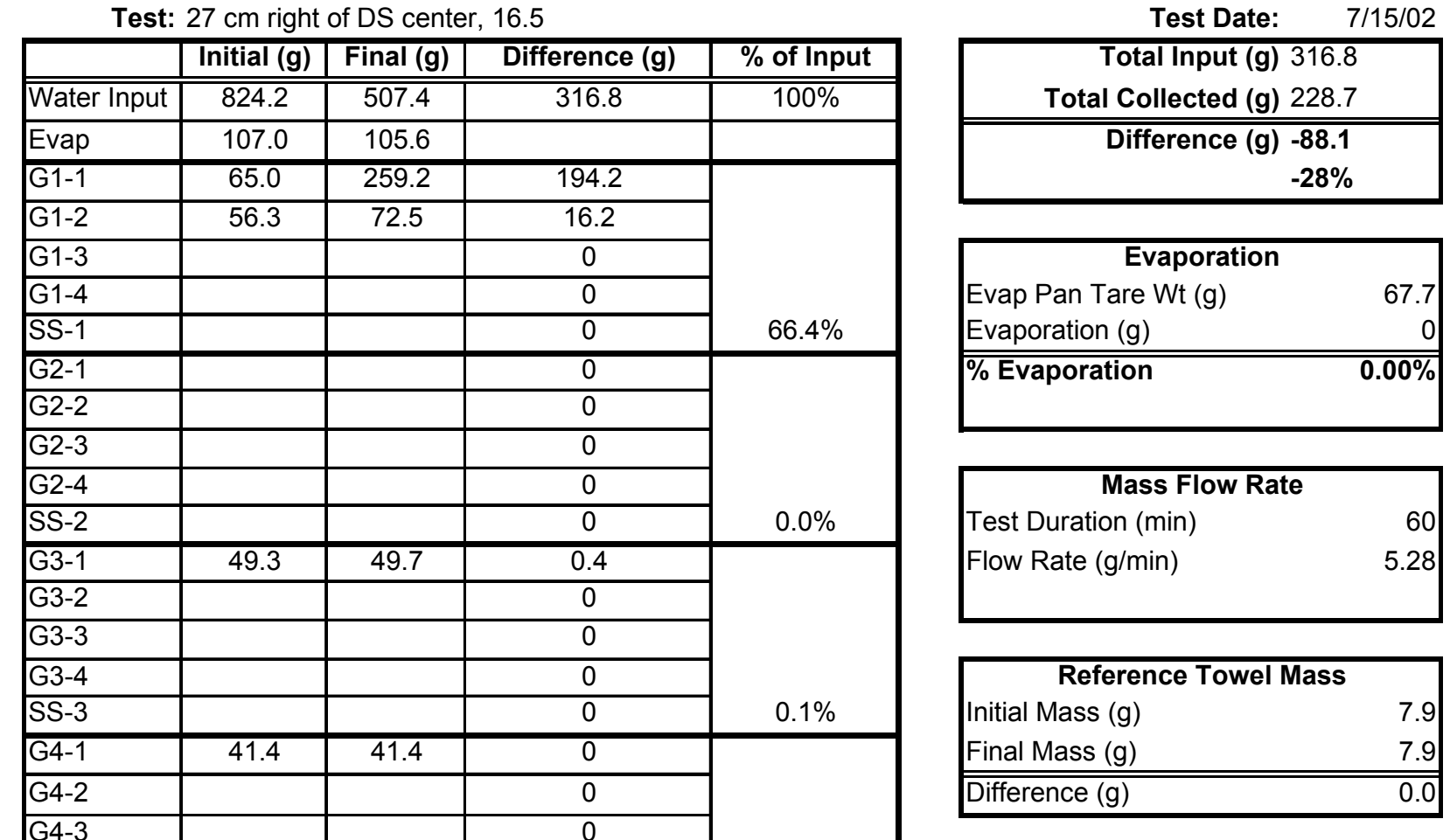

\begin{tabular}{|c|c|c|c|c|}
\hline G4-3 & & & 0 & \multirow[b]{3}{*}{$0.0 \%$} \\
\hline G4-4 & & & 0 & \\
\hline SS-4 & & & 0 & \\
\hline$\overline{B 1}$ & & & 0 & $0.0 \%$ \\
\hline B2 & 106.8 & 106.8 & 0 & $0.0 \%$ \\
\hline B3 & & & 0 & $0.0 \%$ \\
\hline B4 & & & 0 & $0.0 \%$ \\
\hline B5 & 109.0 & 109.4 & 0.4 & $0.1 \%$ \\
\hline$\overline{B 6}$ & 109.8 & 109.5 & -0.3 & $-0.1 \%$ \\
\hline DS in-1 & & & 0 & \multirow[b]{2}{*}{$0.0 \%$} \\
\hline DS in-2 & & & 0 & \\
\hline DS out-1 & 64.3 & 82.1 & 17.8 & \multirow[b]{4}{*}{$5.6 \%$} \\
\hline DS out-2 & & & 0 & \\
\hline DS out-3 & & & 0 & \\
\hline DS out-4 & & & 0 & \\
\hline Other & & & 0 & $0.0 \%$ \\
\hline
\end{tabular}

Total Collected

228.7

$72.2 \%$

\begin{tabular}{|lcccc|}
\hline \multicolumn{6}{l}{ Test Chamber Environment } & & \\
\hline Initial Conditions & $\mathrm{RH}(\%)$ & Temperature $\left({ }^{\circ} \mathrm{C}\right)$ & Atm. P. (mbar) \\
1-HUM/TMP-BDST & 89.7 & 26 & P-IN-BDST & 854 \\
2-HUM/TMP-BDST & 90.9 & 25.7 & & Atm. P. (mbar) \\
\hline Final Conditions & $\mathrm{RH}(\%)$ & Temperature $\left({ }^{\circ} \mathrm{C}\right)$ & & 865 \\
1-HUM/TMP-BDST & 91.9 & 26.8 & P-IN-BDST & \\
2-HUM/TMP-BDST & 92.8 & 26.6 & & \\
\hline
\end{tabular}


Multiple Patch Test Summary and Mass Balance - Rough Surface

Test: $27 \mathrm{~cm}$ left of DS center, 16.5

Test Date: $\quad 7 / 15 / 02$

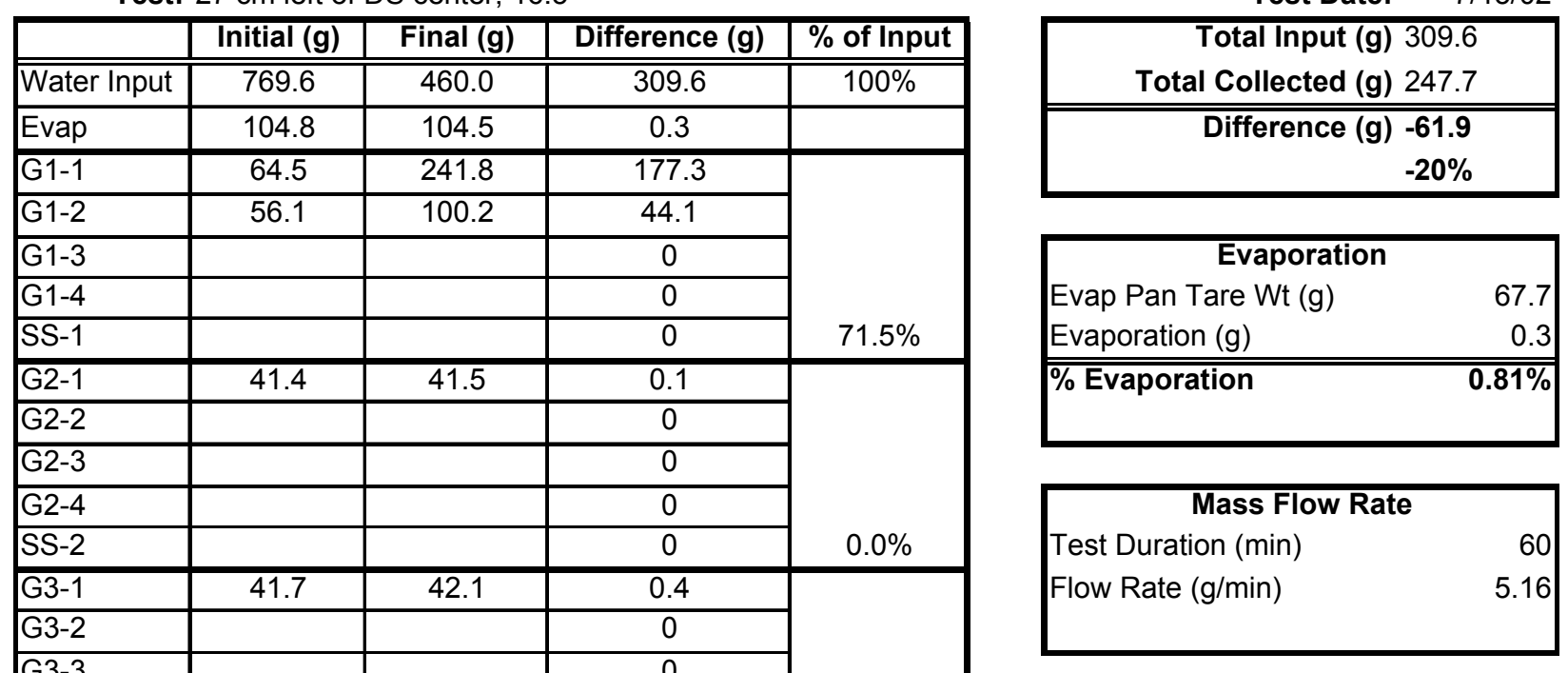

\begin{tabular}{|c|c|c|c|c|}
\hline-5 & & & & \multirow[b]{3}{*}{$0.1 \%$} \\
\hline G3-4 & & & 0 & \\
\hline SS-3 & & & 0 & \\
\hline G4-1 & & & 0 & \multirow[b]{5}{*}{$0.0 \%$} \\
\hline G4-2 & & & 0 & \\
\hline G4-3 & & & 0 & \\
\hline G4-4 & & & 0 & \\
\hline SS-4 & & & 0 & \\
\hline$\overline{B 1}$ & & & 0 & $0.0 \%$ \\
\hline B2 & 106.6 & 107.1 & 0.5 & $0.2 \%$ \\
\hline B3 & & & 0 & $0.0 \%$ \\
\hline B4 & 105.8 & 106.3 & 0.5 & $0.2 \%$ \\
\hline B5 & 109.0 & 109.5 & 0.5 & $0.2 \%$ \\
\hline B6 & 109.8 & 110.5 & 0.7 & $0.2 \%$ \\
\hline DS in-1 & & & 0 & \multirow[b]{2}{*}{$0.0 \%$} \\
\hline DS in-2 & & & 0 & \\
\hline DS out-1 & 64.8 & 88.4 & 23.6 & \multirow[b]{4}{*}{$7.6 \%$} \\
\hline DS out-2 & & & 0 & \\
\hline DS out-3 & & & 0 & \\
\hline DS out-4 & & & 0 & \\
\hline Other & & & 0 & $0.0 \%$ \\
\hline
\end{tabular}

\begin{tabular}{|lr|}
\hline \multicolumn{2}{|c|}{ Reference Towel Mass } \\
Initial Mass $(\mathrm{g})$ & 7.7 \\
Final Mass $(\mathrm{g})$ & 8.0 \\
\hline \hline Difference $(\mathrm{g})$ & 0.3 \\
\hline
\end{tabular}

\begin{tabular}{|lcccc|}
\hline \multicolumn{5}{l}{ Test Chamber Environment } \\
\hline Initial Conditions & $\mathrm{RH}(\%)$ & Temperature $\left({ }^{\circ} \mathrm{C}\right)$ & Atm. P. (mbar) \\
1-HUM/TMP-BDST & 87.9 & 26.1 & P-IN-BDST & 859 \\
2-HUM/TMP-BDST & 87.4 & 25.9 & & \\
\hline Final Conditions & $\mathrm{RH}(\%)$ & Temperature $\left({ }^{\circ} \mathrm{C}\right)$ & Atm. P. (mbar) \\
1-HUM/TMP-BDST & 92.9 & 27.6 & P-IN-BDST & 864 \\
2-HUM/TMP-BDST & 92.8 & 27.2 & & \\
\hline
\end{tabular}


Multiple Patch Test Summary and Mass Balance - Rough Surface

Test: $81 \mathrm{~cm}$ left of DS center, 16.5

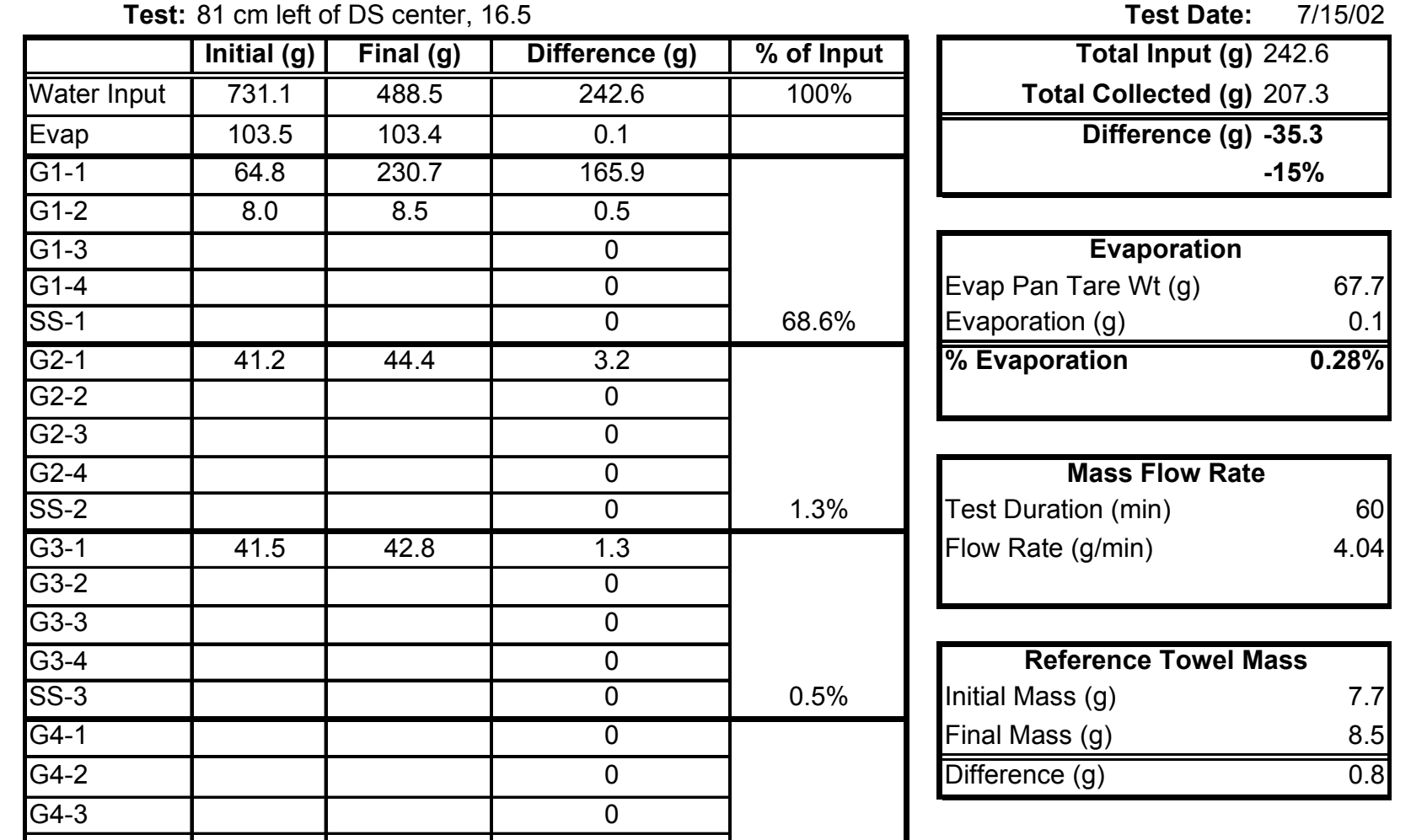

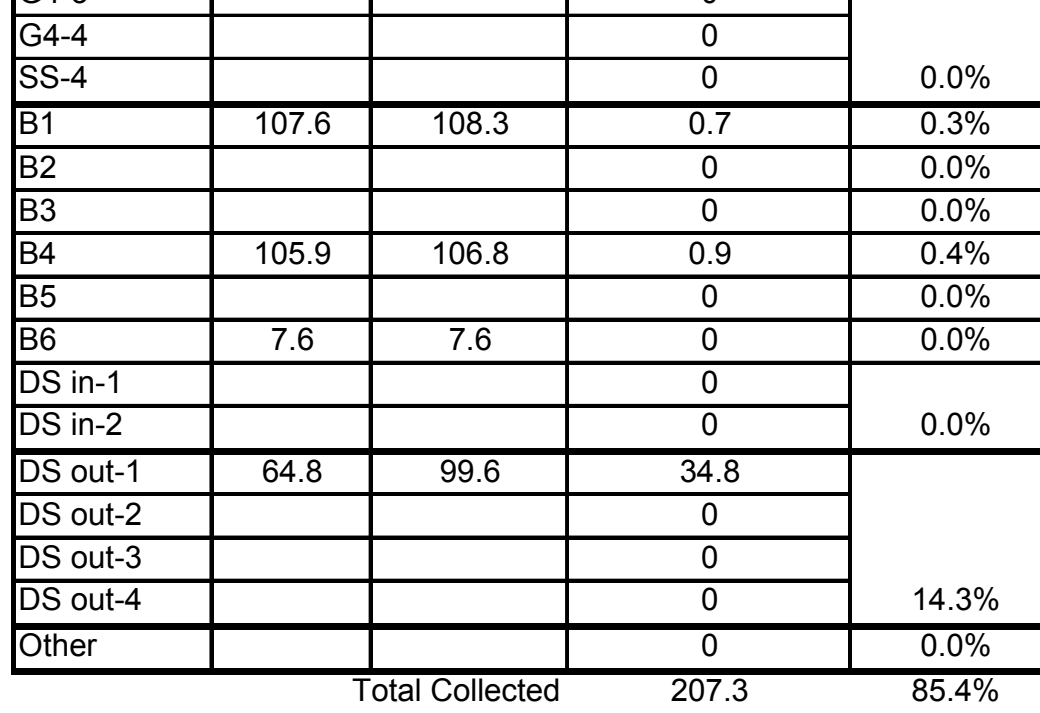

\begin{tabular}{|lcccc|}
\hline \multicolumn{5}{ll}{ Test Chamber Environment } \\
\hline Initial Conditions & $\mathrm{RH}(\%)$ & Temperature $\left({ }^{\circ} \mathrm{C}\right)$ & Atm. P. (mbar) \\
1-HUM/TMP-BDST & 85.2 & 25.7 & P-IN-BDST & 860 \\
2-HUM/TMP-BDST & 86.6 & 25.5 & & \\
\hline Final Conditions & $\mathrm{RH}(\%)$ & Temperature $\left({ }^{\circ} \mathrm{C}\right)$ & Atm. P. (mbar) \\
1-HUM/TMP-BDST & 94.7 & 28.3 & P-IN-BDST & 866 \\
2-HUM/TMP-BDST & 94.6 & 27.9 & & \\
\hline
\end{tabular}


Multiple Patch Test Summary and Mass Balance - Rough Surface

Test: $81 \mathrm{~cm}$ left of DS center, 33

Test Date: $\quad 7 / 16 / 02$

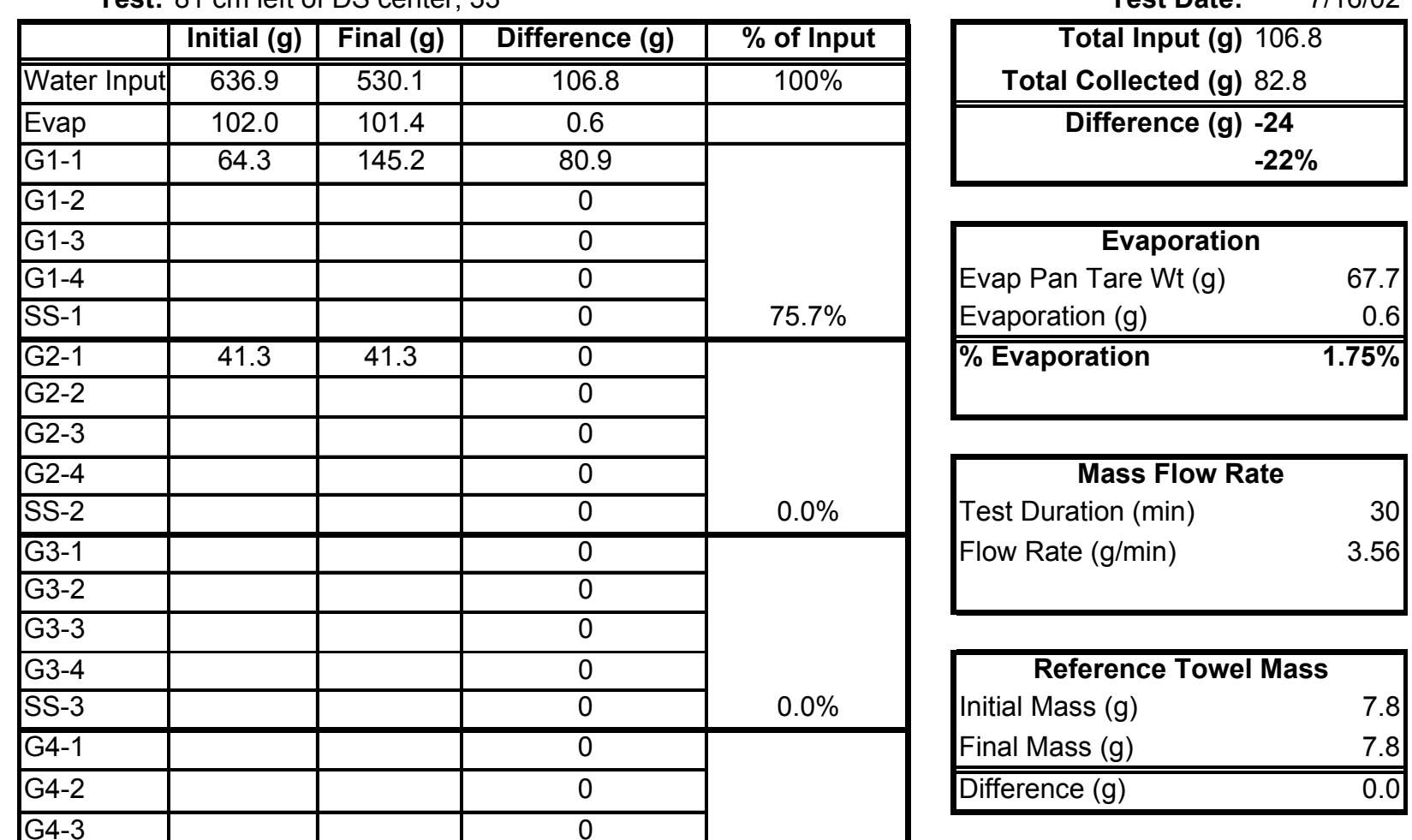

\begin{tabular}{|c|c|c|c|c|}
\hline ע-דל & & & 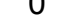 & \multirow[b]{3}{*}{$0.0 \%$} \\
\hline G4-4 & & & 0 & \\
\hline SS-4 & & & 0 & \\
\hline B1 & & & 0 & $0.0 \%$ \\
\hline B2 & & & 0 & $0.0 \%$ \\
\hline B3 & & & 0 & $0.0 \%$ \\
\hline B4 & 106.0 & 106.0 & 0 & $0.0 \%$ \\
\hline B5 & & & 0 & $0.0 \%$ \\
\hline B6 & & & 0 & $0.0 \%$ \\
\hline DS in-1 & & & 0 & \multirow[b]{2}{*}{$0.0 \%$} \\
\hline DS in-2 & & & 0 & \\
\hline DS out-1 & 49.9 & 51.8 & 1.9 & \multirow[b]{4}{*}{$1.8 \%$} \\
\hline DS out-2 & & & 0 & \\
\hline DS out-3 & & & 0 & \\
\hline DS out-4 & & & 0 & \\
\hline Other & & & 0 & $0.0 \%$ \\
\hline
\end{tabular}

\begin{tabular}{|lcccc|}
\hline \multicolumn{5}{ll}{ Test Chamber Environment } \\
\hline Initial Conditions & $\mathrm{RH}(\%)$ & Temperature $\left({ }^{\circ} \mathrm{C}\right)$ & Atm. P. (mbar) \\
1-HUM/TMP-BDST & 83.1 & 25.7 & $\mathrm{P}-\mathrm{IN}-\mathrm{mDST}$ & 854 \\
2-HUM/TMP-BDST & 84.6 & 25.6 & & \\
\hline Final Conditions & $\mathrm{RH}(\%)$ & Temperature $\left({ }^{\circ} \mathrm{C}\right)$ & Atm. P. (mbar) \\
1-HUM/TMP-BDST & 83.4 & 26.1 & P-IN-BDST & 847 \\
2-HUM/TMP-BDST & 83.8 & 26 & & \\
\hline
\end{tabular}


Multiple Patch Test Summary and Mass Balance - Rough Surface

Test: $27 \mathrm{~cm}$ left of DS center, 33

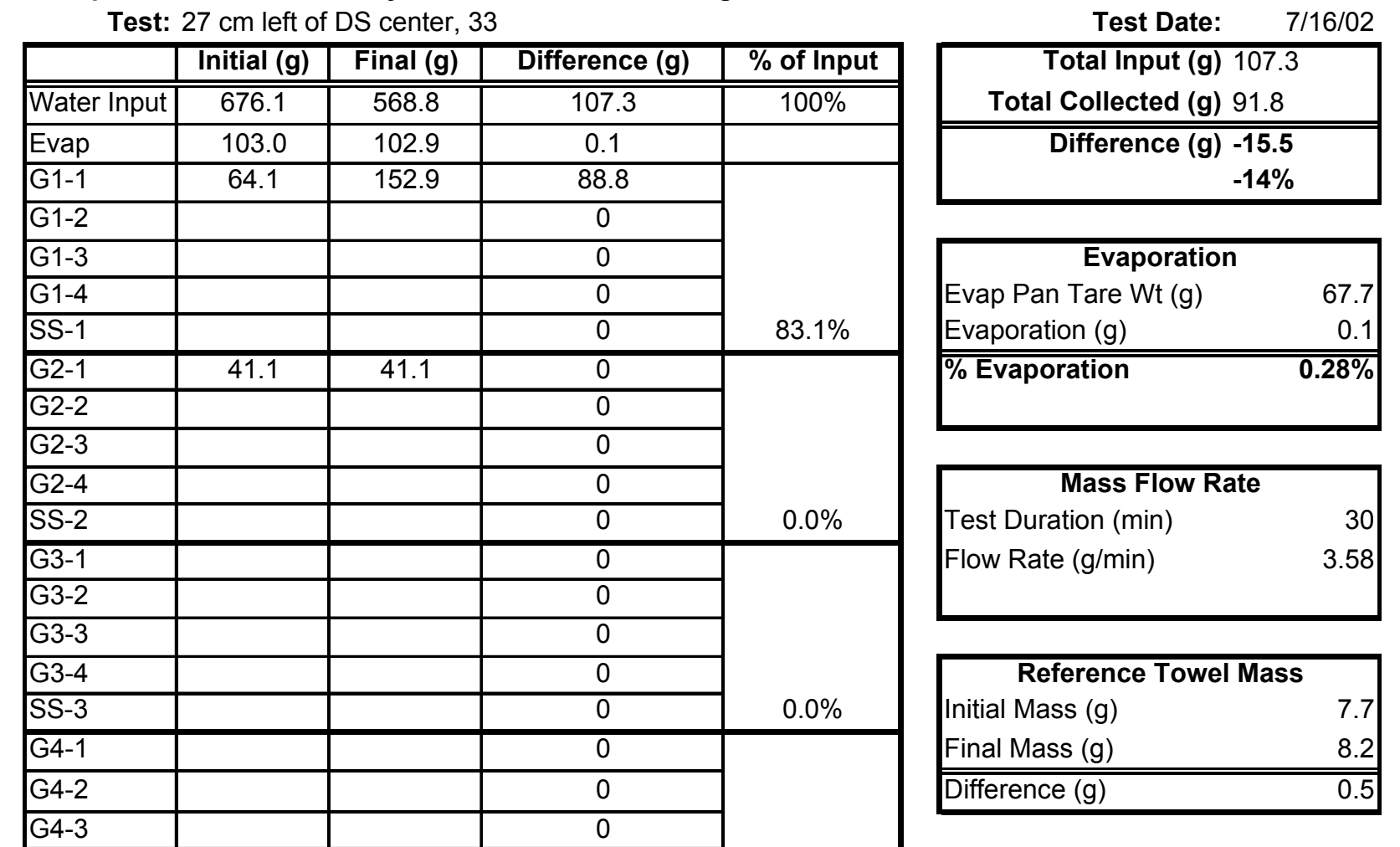

\begin{tabular}{|c|c|c|c|c|}
\hline G4-4 & & & 0 & \multirow[b]{2}{*}{$0.0 \%$} \\
\hline SS-4 & & & 0 & \\
\hline B1 & & & 0 & $0.0 \%$ \\
\hline B2 & & & 0 & $0.0 \%$ \\
\hline B3 & & & 0 & $0.0 \%$ \\
\hline B4 & 106.1 & 106.4 & 0.3 & $0.3 \%$ \\
\hline B5 & & & 0 & $0.0 \%$ \\
\hline B6 & & & 0 & $0.0 \%$ \\
\hline DS in-1 & & & 0 & \multirow[b]{2}{*}{$0.0 \%$} \\
\hline DS in-2 & & & 0 & \\
\hline DS out-1 & 49.8 & 52.5 & 2.7 & \multirow[b]{4}{*}{$2.5 \%$} \\
\hline DS out- 2 & & & 0 & \\
\hline DS out-3 & & & 0 & \\
\hline DS out-4 & & & 0 & \\
\hline Other & & & 0 & $0.0 \%$ \\
\hline
\end{tabular}

\begin{tabular}{|lcccc|}
\hline \multicolumn{5}{l}{ Test Chamber Environment } \\
\hline Initial Conditions & $\mathrm{RH}(\%)$ & Temperature $\left({ }^{\circ} \mathrm{C}\right)$ & Atm. P. (mbar) \\
1-HUM/TMP-BDST & 84.2 & 25.3 & P-IN-BDST & 857 \\
2-HUM/TMP-BDST & 86 & 25.2 & & \\
\hline Final Conditions & $\mathrm{RH}(\%)$ & Temperature $\left({ }^{\circ} \mathrm{C}\right)$ & Atm. P. (mbar) \\
1-HUM/TMP-BDST & 92.8 & 27.4 & P-IN-BDST & 853 \\
2-HUM/TMP-BDST & 93.3 & 27.1 & & \\
\hline
\end{tabular}


Multiple Patch Test Summary and Mass Balance - Rough Surface

Test: $27 \mathrm{~cm}$ right of DS center, 33

\begin{tabular}{|c|c|c|c|c|}
\hline & Initial & Final & Difference & $\%$ of Input \\
\hline Water Input & 690.9 & 582.5 & 108.4 & 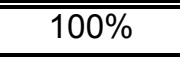 \\
\hline Evap & 104.0 & 103.5 & 0.5 & \\
\hline G1-1 & 65.1 & 152.0 & 86.9 & \multirow[b]{5}{*}{$80.2 \%$} \\
\hline G1-2 & & & 0 & \\
\hline G1-3 & & & 0 & \\
\hline G1-4 & & & 0 & \\
\hline SS-1 & & & 0 & \\
\hline G2-1 & & & 0 & \multirow[b]{5}{*}{$0.0 \%$} \\
\hline G2-2 & & & 0 & \\
\hline G2-3 & & & 0 & \\
\hline G2-4 & & & 0 & \\
\hline SS-2 & & & 0 & \\
\hline G3-1 & & & 0 & \multirow[b]{5}{*}{$0.0 \%$} \\
\hline G3-2 & & & 0 & \\
\hline G3-3 & & & 0 & \\
\hline G3-4 & & & 0 & \\
\hline SS-3 & & & 0 & \\
\hline G4-1 & 41.1 & 41.1 & 0 & \multirow[b]{5}{*}{$0.0 \%$} \\
\hline G4-2 & & & 0 & \\
\hline G4-3 & & & 0 & \\
\hline G4-4 & & & 0 & \\
\hline SS-4 & & & 0 & \\
\hline $\mathrm{B} 1$ & & & 0 & $0.0 \%$ \\
\hline B2 & & & 0 & $0.0 \%$ \\
\hline B3 & & & 0 & $0.0 \%$ \\
\hline B4 & & & 0 & $0.0 \%$ \\
\hline B5 & 109.4 & 109.7 & 0.3 & $0.3 \%$ \\
\hline B6 & & & 0 & $0.0 \%$ \\
\hline DS in-1 & & & 0 & \multirow[b]{2}{*}{$0.0 \%$} \\
\hline DS in-2 & & & 0 & \\
\hline DS out-1 & 50.6 & 52.1 & 1.5 & \multirow[b]{4}{*}{$1.4 \%$} \\
\hline DS out-2 & & & 0 & \\
\hline DS out-3 & & & 0 & \\
\hline DS out-4 & & & 0 & \\
\hline Other & & & 0 & $0.0 \%$ \\
\hline
\end{tabular}

Test Date: $\quad 7 / 16 / 02$

\begin{tabular}{|r|}
\hline Total Input 108.4 \\
Total Collected 88.7 \\
\hline \hline Difference -19.7 \\
$-18 \%$ \\
\hline
\end{tabular}

\begin{tabular}{|lr|}
\hline \multicolumn{2}{|c|}{ Evaporation } \\
Evap Pan Tare Wt, g & 66.7 \\
Evaporation, g & 0.5 \\
\hline \% Evaporation & $\mathbf{1 . 3 4 \%}$ \\
\hline
\end{tabular}

Mass Flow Rate

Test Duration, min

Flow Rate, g/min

\begin{tabular}{|lr|}
\hline \multicolumn{2}{|c|}{ Reference Towel Mass } \\
Initial Mass $(\mathrm{g})$ & 7.7 \\
Final Mass $(\mathrm{g})$ & 8.0 \\
\hline \hline Difference $(\mathrm{g})$ & 0.3 \\
\hline
\end{tabular}

\begin{tabular}{|lcccc|}
\hline \multicolumn{5}{l}{ Test Chamber Environment } \\
\hline Initial Conditions & $\mathrm{RH}(\%)$ & Temperature $\left({ }^{\circ} \mathrm{C}\right)$ & Atm. P. (mbar) \\
1-HUM/TMP-BDST & 86.7 & 25.1 & P-IN-BDST & 854 \\
2-HUM/TMP-BDST & 86.1 & 25.1 & & \\
\hline Final Conditions & $\mathrm{RH}(\%)$ & Temperature $\left({ }^{\circ} \mathrm{C}\right)$ & Atm. P. (mbar) \\
1-HUM/TMP-BDST & 92.1 & 26.4 & P-IN-BDST & 864 \\
2-HUM/TMP-BDST & 91.5 & 26.4 & & \\
\hline
\end{tabular}


Multiple Patch Test Summary and Mass Balance - Rough Surface

Test: $81 \mathrm{~cm}$ right of DS center, 33

\begin{tabular}{|c|c|c|c|c|}
\hline & Initial & Final & Difference & $\%$ of Input \\
\hline Water Input & 652.0 & $\overline{542.6}$ & 109.4 & $100 \%$ \\
\hline Evap & 105.9 & 105.0 & 0.9 & \\
\hline G1-1 & 65.1 & 149.7 & 84.6 & \multirow[b]{5}{*}{$77.3 \%$} \\
\hline$\overline{\mathrm{G} 1-2}$ & & & 0 & \\
\hline$\overline{G 1-3}$ & & & 0 & \\
\hline G1-4 & & & 0 & \\
\hline SS-1 & & & 0 & \\
\hline $\mathrm{G} 2-1$ & & & 0 & \multirow[b]{5}{*}{$0.0 \%$} \\
\hline$\overline{\mathrm{G} 2-2}$ & & & 0 & \\
\hline G2-3 & & & 0 & \\
\hline G2-4 & & & 0 & \\
\hline SS-2 & & & 0 & \\
\hline G3-1 & & & 0 & \multirow[b]{5}{*}{$0.0 \%$} \\
\hline G3-2 & & & 0 & \\
\hline G3-3 & & & 0 & \\
\hline G3-4 & & & 0 & \\
\hline SS-3 & & & 0 & \\
\hline$\overline{\mathrm{G} 4-1}$ & 41.3 & 41.6 & 0.3 & \multirow[b]{5}{*}{$0.3 \%$} \\
\hline G4-2 & & & 0 & \\
\hline G4-3 & & & 0 & \\
\hline G4-4 & & & 0 & \\
\hline SS-4 & & & 0 & \\
\hline$B 1$ & & & 0 & $0.0 \%$ \\
\hline B2 & & & 0 & $0.0 \%$ \\
\hline B3 & 107.7 & 107.8 & 0.1 & $0.1 \%$ \\
\hline B4 & & & 0 & $0.0 \%$ \\
\hline B5 & 109.3 & 109.5 & 0.2 & $0.2 \%$ \\
\hline B6 & & & 0 & $0.0 \%$ \\
\hline DS in-1 & & & 0 & \multirow[b]{2}{*}{$0.0 \%$} \\
\hline DS in-2 & & & 0 & \\
\hline DS out-1 & 56.9 & 58.3 & 1.4 & \multirow[b]{4}{*}{$1.3 \%$} \\
\hline DS out- 2 & & & 0 & \\
\hline DS out-3 & & & 0 & \\
\hline DS out-4 & & & 0 & \\
\hline Other & & & 0 & $0.0 \%$ \\
\hline
\end{tabular}

Test Date: $\quad 7 / 16 / 02$

Total Input 109.4

Total Collected 86.6

Difference -22.8 $-21 \%$

\section{Evaporation}

Evap Pan Tare Wt, g

66.7

Evaporation, $\mathrm{g}$

0.9

\% Evaporation

$2.30 \%$

\begin{tabular}{|lr|}
\hline \multicolumn{2}{|c|}{ Mass Flow Rate } \\
Test Duration, min & 30.03 \\
Flow Rate, g/min & 3.64 \\
& \\
\hline
\end{tabular}

\section{Reference Towel Mass}

Initial Mass $(\mathrm{g})$

Final Mass (g)

7.8

Difference (g)

$-0.1$

\begin{tabular}{|lcccc|}
\hline \multicolumn{7}{|l|}{ Test Chamber Environment } & & & \\
\hline Initial Conditions & $\mathrm{RH}(\%)$ & Temperature $\left({ }^{\circ} \mathrm{C}\right)$ & Atm. P. (mbar) \\
1-HUM/TMP-BDST & 86 & 25.1 & P-IN-BDST & 860 \\
2-HUM/TMP-BDST & 85.1 & 25.5 & & \\
\hline Final Conditions & $\mathrm{RH}(\%)$ & Temperature $\left({ }^{\circ} \mathrm{C}\right)$ & & Atm. P. (mbar) \\
1-HUM/TMP-BDST & 88.6 & 25.9 & P-IN-BDST & 864 \\
2-HUM/TMP-BDST & 88.4 & 25.4 & & \\
\hline
\end{tabular}

SN-M\&O-SCI-043-V2, pages 167-168 
Bounding Flow Test Summary and Mass Balance - Rough DS Surface

Test: High flow rate, $54 \mathrm{~cm}$ left of DS center, Crown

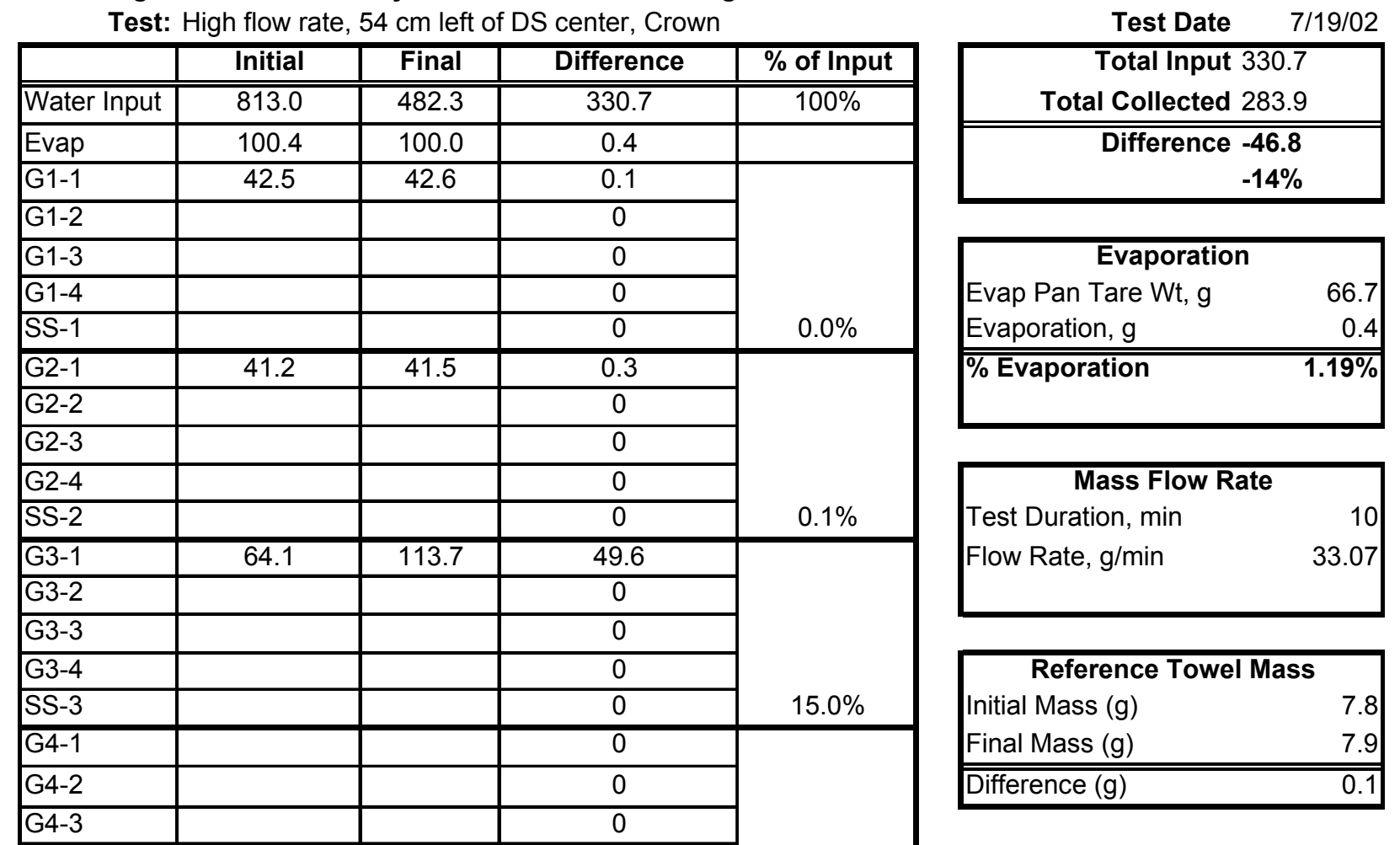

\begin{tabular}{|c|c|c|c|c|}
\hline & & & & \multirow[b]{3}{*}{$0.0 \%$} \\
\hline G4-4 & & & 0 & \\
\hline SS-4 & & & 0 & \\
\hline B1 & & & 0 & $0.0 \%$ \\
\hline B2 & & & 0 & $0.0 \%$ \\
\hline B3 & & & 0 & $0.0 \%$ \\
\hline B4 & 107.4 & 301.3 & 193.9 & $58.6 \%$ \\
\hline B5 & & & 0 & $0.0 \%$ \\
\hline B6 & 102.2 & 102.9 & 0.7 & $0.2 \%$ \\
\hline DS in-1 & & & 0 & \multirow[b]{2}{*}{$0.0 \%$} \\
\hline DS in-2 & & & 0 & \\
\hline DS out-1 & 71.8 & 111.1 & 39.3 & \multirow[b]{4}{*}{$11.9 \%$} \\
\hline DS out-2 & & & 0 & \\
\hline DS out-3 & & & 0 & \\
\hline DS out-4 & & & 0 & \\
\hline Other & & & 0 & $0.0 \%$ \\
\hline
\end{tabular}

\begin{tabular}{|lcccc|}
\hline Test Chamber Environment & \multicolumn{4}{c|}{} \\
\hline Initial Conditions & $\mathrm{RH}(\%)$ & Temperature $\left({ }^{\circ} \mathrm{C}\right)$ & Atm. P. (mbar) \\
1-HUM/TMP-BDST & 84.4 & 24.8 & P-IN-BDST & 863 \\
2-HUM/TMP-BDST & 85 & 25 & & \\
\hline Final Conditions & $\mathrm{RH}(\%)$ & Temperature $\left({ }^{\circ} \mathrm{C}\right)$ & Atm. P. (mbar) \\
1-HUM/TMP-BDST & 90.3 & 25.4 & P-IN-BDST & 861 \\
2-HUM/TMP-BDST & 90.8 & 25.2 & & \\
\hline
\end{tabular}


Bounding Flow Test Summary and Mass Balance - Rough DS Surface

Test: Low flow rate, $54 \mathrm{~cm}$ left of DS Center, Crown

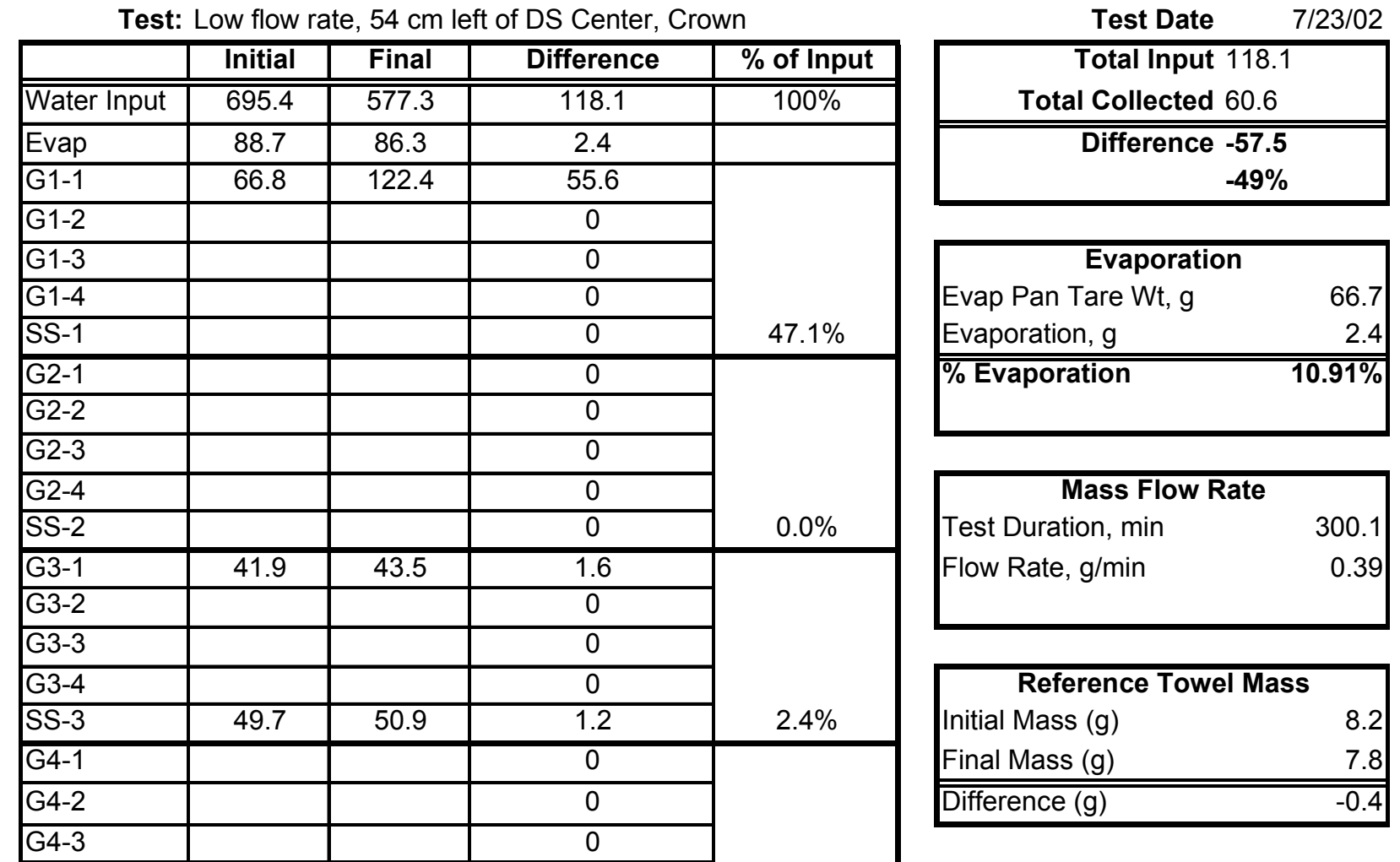

\begin{tabular}{|c|c|c|c|c|}
\hline & & & & \multirow[b]{3}{*}{$0.0 \%$} \\
\hline G4-4 & & & 0 & \\
\hline SS-4 & & & 0 & \\
\hline B1 & 106.4 & 106.1 & 0 & $0.0 \%$ \\
\hline B2 & 108.2 & 107.1 & 0 & $0.0 \%$ \\
\hline B3 & & & 0 & $0.0 \%$ \\
\hline B4 & 108.4 & 107.5 & 0 & $0.0 \%$ \\
\hline B5 & & & 0 & $0.0 \%$ \\
\hline B6 & 110.4 & 110.1 & 0 & $0.0 \%$ \\
\hline DS in-1 & & & 0 & \multirow[b]{2}{*}{$0.0 \%$} \\
\hline DS in-2 & & & 0 & \\
\hline DS out-1 & 66.8 & 69.0 & 2.2 & \multirow[b]{4}{*}{$1.9 \%$} \\
\hline DS out- 2 & & & 0 & \\
\hline DS out-3 & & & 0 & \\
\hline DS out-4 & & & 0 & \\
\hline Other & & & 0 & $0.0 \%$ \\
\hline
\end{tabular}

\begin{tabular}{|lcccc|}
\hline \multicolumn{4}{l}{ Test Chamber Environment } \\
\hline Initial Conditions & $\mathrm{RH}(\%)$ & Temperature $\left({ }^{\circ} \mathrm{C}\right)$ & Atm. P. (mbar) \\
1-HUM/TMP-BDST & 101.7 & 26.9 & P-IN-BDST & 854 \\
2-HUM/TMP-BDST & 92.7 & 26.2 & & \\
\hline Final Conditions & $\mathrm{RH}(\%)$ & Temperature $\left({ }^{\circ} \mathrm{C}\right)$ & Atm. P. (mbar) \\
1-HUM/TMP-BDST & 88.4 & 27.2 & P-IN-BDST & 858 \\
2-HUM/TMP-BDST & 91.2 & 26.6 & & \\
\hline
\end{tabular}


Bounding Flow Test Summary and Mass Balance - Rough DS Surface

Test: High flow rate, $27 \mathrm{~cm}$ left of DS center, Crown

\begin{tabular}{|c|c|c|c|c|}
\hline & Initial & Final & Difference & $\%$ of Input \\
\hline Water Input & 788.1 & 459.5 & 328.6 & $100 \%$ \\
\hline Evap & 99.8 & 99.4 & 0.4 & \\
\hline G1-1 & 64.7 & 197.6 & 132.9 & \multirow[b]{5}{*}{$40.4 \%$} \\
\hline G1-2 & & & 0 & \\
\hline G1-3 & & & 0 & \\
\hline G1-4 & & & 0 & \\
\hline SS-1 & & & 0 & \\
\hline G2-1 & 41.5 & 41.5 & 0 & \multirow[b]{5}{*}{$0.0 \%$} \\
\hline G2-2 & & & 0 & \\
\hline G2-3 & & & 0 & \\
\hline G2-4 & & & 0 & \\
\hline SS-2 & & & 0 & \\
\hline G3-1 & 63.9 & 144.8 & 80.9 & \multirow[b]{5}{*}{$24.6 \%$} \\
\hline G3-2 & & & 0 & \\
\hline G3-3 & & & 0 & \\
\hline G3-4 & & & 0 & \\
\hline SS-3 & & & 0 & \\
\hline G4-1 & & & 0 & \multirow[b]{5}{*}{$0.0 \%$} \\
\hline G4-2 & & & 0 & \\
\hline G4-3 & & & 0 & \\
\hline G4-4 & & & 0 & \\
\hline SS-4 & & & 0 & \\
\hline B1 & & & 0 & $0.0 \%$ \\
\hline B2 & 7.6 & 10.5 & 2.9 & $0.9 \%$ \\
\hline B3 & & & 0 & $0.0 \%$ \\
\hline B4 & 99.7 & 100.3 & 0.6 & $0.2 \%$ \\
\hline B5 & & & 0 & $0.0 \%$ \\
\hline B6 & 109.9 & 112.2 & 2.3 & $0.7 \%$ \\
\hline DS in-1 & & & 0 & \multirow[b]{2}{*}{$0.0 \%$} \\
\hline DS in-2 & & & 0 & \\
\hline DS out-1 & 72.0 & 127.9 & 55.9 & \multirow[b]{4}{*}{$17.0 \%$} \\
\hline DS out-2 & & & 0 & \\
\hline DS out-3 & & & 0 & \\
\hline DS out-4 & & & 0 & \\
\hline Other & & & 0 & $0.0 \%$ \\
\hline
\end{tabular}

Test Date $\quad 7 / 19 / 02$

Total Input 328.6

Total Collected 275.5

Difference -53.1

$-16 \%$

Evaporation

Evap Pan Tare Wt, g

Evaporation, g

\% Evaporation $1.21 \%$

Mass Flow Rate

Test Duration, min

10

Flow Rate, $\mathrm{g} / \mathrm{min}$

\begin{tabular}{|lr|}
\hline \multicolumn{2}{|c|}{ Reference Towel Mass } \\
Initial Mass $(\mathrm{g})$ & 7.8 \\
Final Mass $(\mathrm{g})$ & 8.0 \\
\hline \hline Difference $(\mathrm{g})$ & 0.2 \\
\hline
\end{tabular}

\begin{tabular}{|lcccc|}
\hline Test Chamber Environment & & & \\
\hline Initial Conditions & $\mathrm{RH}(\%)$ & Temperature $\left({ }^{\circ} \mathrm{C}\right)$ & Atm. P. (mbar) \\
1-HUM/TMP-BDST & 89 & 24.8 & P-IN-BDST & 855 \\
2-HUM/TMP-BDST & 86.4 & 25 & & \\
\hline Final Conditions & $\mathrm{RH}(\%)$ & Temperature $\left({ }^{\circ} \mathrm{C}\right)$ & Atm. P. (mbar) \\
1-HUM/TMP-BDST & 92.2 & 25.9 & P-IN-BDST & 857 \\
2-HUM/TMP-BDST & 92.2 & 25.2 & & \\
\hline
\end{tabular}


Bounding Flow Test Summary and Mass Balance - Rough DS Surface

Test: Low flow rate, $27 \mathrm{~cm}$ left of DS Center, Crown

\begin{tabular}{|c|c|c|c|c|}
\hline & Initial & Final & Difference & $\%$ of Input \\
\hline Water Input & 815.2 & 701.9 & 113.3 & $100 \%$ \\
\hline Evap & 89.1 & 88.9 & 0.2 & \\
\hline G1-1 & 58.0 & 71.5 & 13.5 & \multirow[b]{5}{*}{$11.9 \%$} \\
\hline G1-2 & & & 0 & \\
\hline G1-3 & & & 0 & \\
\hline G1-4 & & & 0 & \\
\hline SS-1 & & & 0 & \\
\hline G2-1 & & & 0 & \multirow[b]{5}{*}{$0.0 \%$} \\
\hline G2-2 & & & 0 & \\
\hline G2-3 & & & 0 & \\
\hline G2-4 & & & 0 & \\
\hline SS-2 & & & 0 & \\
\hline G3-1 & 42.2 & 53.9 & 11.7 & \multirow[b]{5}{*}{$16.4 \%$} \\
\hline G3-2 & & & 0 & \\
\hline G3-3 & & & 0 & \\
\hline G3-4 & & & 0 & \\
\hline SS-3 & 49.6 & 56.5 & 6.9 & \\
\hline G4-1 & & & 0 & \multirow[b]{5}{*}{$0.0 \%$} \\
\hline G4-2 & & & 0 & \\
\hline G4-3 & & & 0 & \\
\hline G4-4 & & & 0 & \\
\hline SS-4 & & & 0 & \\
\hline B1 & & & 0 & $0.0 \%$ \\
\hline B2 & 107.1 & 108.6 & 1.5 & $1.3 \%$ \\
\hline B3 & & & 0 & $0.0 \%$ \\
\hline B4 & 107.5 & 108.8 & 1.3 & $1.1 \%$ \\
\hline B5 & & & 0 & $0.0 \%$ \\
\hline $\mathrm{B} 6$ & 110.0 & 111.6 & 1.6 & $1.4 \%$ \\
\hline DS in-1 & & & 0 & \multirow[b]{2}{*}{$0.0 \%$} \\
\hline DS in-2 & & & 0 & \\
\hline DS out-1 & 65.8 & 91.7 & 25.9 & \multirow[b]{4}{*}{$22.9 \%$} \\
\hline DS out- 2 & & & 0 & \\
\hline DS out-3 & & & 0 & \\
\hline DS out-4 & & & 0 & \\
\hline Other & & & 0 & $0.0 \%$ \\
\hline
\end{tabular}

Test Date $\quad 7 / 23 / 02$

\begin{tabular}{|r|}
\hline Total Input 113.3 \\
Total Collected 62.4 \\
\hline \hline Difference $\mathbf{- 5 0 . 9}$ \\
$-\mathbf{4 5 \%}$ \\
\hline
\end{tabular}

\begin{tabular}{|lr|}
\hline \multicolumn{2}{|c|}{ Evaporation } \\
Evap Pan Tare Wt, g & 66.7 \\
Evaporation, g & 0.2 \\
\hline \% Evaporation & $\mathbf{0 . 8 9 \%}$ \\
\hline
\end{tabular}

\begin{tabular}{|lr|}
\hline \multicolumn{2}{|c|}{ Mass Flow Rate } \\
Test Duration, min & 302.5 \\
Flow Rate, g/min & 0.37 \\
\hline
\end{tabular}

\section{Reference Towel Mass}

Initial Mass $(\mathrm{g})$

Final Mass $(\mathrm{g}) \quad 8.5$

Difference $(\mathrm{g}) \quad 0.6$

\begin{tabular}{|lcccc|}
\hline \multicolumn{5}{ll}{ Test Chamber Environment } \\
\hline Initial Conditions & $\mathrm{RH}(\%)$ & Temperature $\left({ }^{\circ} \mathrm{C}\right)$ & Atm. P. (mbar) \\
1-HUM/TMP-BDST & 89.3 & 21 & P-IN-BDST & 852 \\
2-HUM/TMP-BDST & 93.4 & 20.5 & & \\
\hline Final Conditions & $\mathrm{RH}(\%)$ & Temperature $\left({ }^{\circ} \mathrm{C}\right)$ & Atm. P. (mbar) \\
1-HUM/TMP-BDST & 110 & 28.1 & P-IN-BDST & 849 \\
2-HUM/TMP-BDST & 95.6 & 27.7 & & \\
\hline
\end{tabular}


Bounding Flow Test Summary and Mass Balance - Rough DS Surface

Test: High flow rate, $27 \mathrm{~cm}$ right of DS center, Crown

\begin{tabular}{|c|c|c|c|c|}
\hline & Initial & Final & Difference & $\%$ of Input \\
\hline Water Input & $\overline{7744.2}$ & 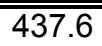 & 306.6 & $\overline{100 \%}$ \\
\hline Evap & 99.0 & 98.5 & 0.5 & \\
\hline$\overline{G 1-1}$ & 64.7 & 229.9 & 165.2 & \multirow[b]{5}{*}{$55.9 \%$} \\
\hline G1-2 & 49.3 & 55.4 & 6.1 & \\
\hline G1-3 & & & 0 & \\
\hline G1-4 & & & 0 & \\
\hline SS-1 & & & 0 & \\
\hline G2-1 & & & 0 & \multirow[b]{5}{*}{$0.0 \%$} \\
\hline G2-2 & & & 0 & \\
\hline G2-3 & & & 0 & \\
\hline G2-4 & & & 0 & \\
\hline SS-2 & & & 0 & \\
\hline G3-1 & 64.0 & 112.4 & 48.4 & \multirow[b]{5}{*}{$15.8 \%$} \\
\hline G3-2 & & & 0 & \\
\hline G3-3 & & & 0 & \\
\hline G3-4 & & & 0 & \\
\hline SS-3 & & & 0 & \\
\hline G4-1 & 41.0 & 41.1 & 0.1 & \multirow[b]{5}{*}{$0.0 \%$} \\
\hline G4-2 & & & 0 & \\
\hline G4-3 & & & 0 & \\
\hline G4-4 & & & 0 & \\
\hline SS-4 & & & 0 & \\
\hline B1 & & & 0 & $0.0 \%$ \\
\hline B2 & 99.1 & 99.2 & 0.1 & $0.0 \%$ \\
\hline B3 & & & 0 & $0.0 \%$ \\
\hline B4 & & & 0 & $0.0 \%$ \\
\hline B5 & 109.1 & 109.4 & 0.3 & $0.1 \%$ \\
\hline B6 & 109.8 & 112.4 & 2.6 & $0.8 \%$ \\
\hline DS in-1 & & & 0 & \multirow[b]{2}{*}{$0.0 \%$} \\
\hline DS in-2 & & & 0 & \\
\hline DS out-1 & 71.7 & 110.8 & 39.1 & \multirow[b]{4}{*}{$12.8 \%$} \\
\hline DS out-2 & & & 0 & \\
\hline DS out-3 & & & 0 & \\
\hline DS out-4 & & & 0 & \\
\hline Other & & & 0 & $0.0 \%$ \\
\hline
\end{tabular}

Test Date $\quad 7 / 19 / 02$

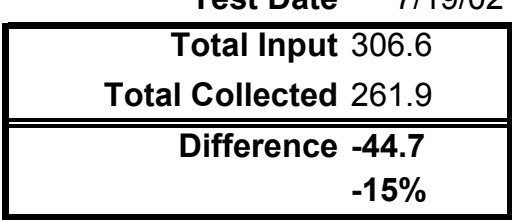

Evaporation

Evap Pan Tare Wt, g $\quad 66.7$

Evaporation, g

\% Evaporation $1.55 \%$

\begin{tabular}{|lr|}
\hline \multicolumn{2}{|c|}{ Mass Flow Rate } \\
Test Duration, min & 10 \\
Flow Rate, g/min & 30.66 \\
\hline
\end{tabular}

\begin{tabular}{|lr|}
\hline \multicolumn{2}{|c|}{ Reference Towel Mass } \\
Initial Mass $(\mathrm{g})$ & 7.7 \\
Final Mass $(\mathrm{g})$ & 7.8 \\
\hline \hline Difference $(\mathrm{g})$ & 0.1 \\
\hline
\end{tabular}

\begin{tabular}{|lcccc|}
\hline \multicolumn{4}{ll}{ Test Chamber Environment } \\
\hline Initial Conditions & $\mathrm{RH}(\%)$ & Temperature $\left({ }^{\circ} \mathrm{C}\right)$ & Atm. P. (mbar) \\
1-HUM/TMP-BDST & 87.5 & 24 & P-IN-BDST & 856 \\
2-HUM/TMP-BDST & 81.4 & 25 & & \\
\hline Final Conditions & $\mathrm{RH}(\%)$ & Temperature $\left({ }^{\circ} \mathrm{C}\right)$ & Atm. P. (mbar) \\
1-HUM/TMP-BDST & 91 & 25.9 & P-IN-BDST & 852 \\
2-HUM/TMP-BDST & 91.3 & 25.4 & & \\
\hline
\end{tabular}

SN-M\&O-SCl-043-V2, pages 198-199 
Bounding Flow Test Summary and Mass Balance - Rough DS Surface

Test: low flow, $27 \mathrm{~cm}$ right of DS center, Crown

\begin{tabular}{|c|c|c|c|c|}
\hline & Initial & Final & Difference & $\%$ of Input \\
\hline Water Input & 7444.8 & 2674.0 & 70.8 & $100 \%$ \\
\hline Evap & 96.2 & 95.1 & 1.1 & \\
\hline G1-1 & 66.0 & 95.1 & 29.1 & \multirow[b]{5}{*}{$41.1 \%$} \\
\hline G1-2 & & & 0 & \\
\hline G1-3 & & & 0 & \\
\hline G1-4 & & & 0 & \\
\hline SS-1 & & & 0 & \\
\hline G2-1 & & & 0 & \multirow[b]{5}{*}{$0.0 \%$} \\
\hline G2-2 & & & 0 & \\
\hline G2-3 & & & 0 & \\
\hline G2-4 & & & 0 & \\
\hline SS-2 & & & 0 & \\
\hline G3-1 & 50.0 & 52.6 & 2.6 & \multirow[b]{5}{*}{$6.1 \%$} \\
\hline G3-2 & & & 0 & \\
\hline G3-3 & & & 0 & \\
\hline G3-4 & & & 0 & \\
\hline SS-3 & 49.3 & 51.0 & 1.7 & \\
\hline G4-1 & & & 0 & \multirow[b]{5}{*}{$0.0 \%$} \\
\hline G4-2 & & & 0 & \\
\hline G4-3 & & & 0 & \\
\hline G4-4 & & & 0 & \\
\hline SS-4 & & & 0 & \\
\hline B1 & & & 0 & $0.0 \%$ \\
\hline B2 & 111.6 & 110.4 & 0 & $0.0 \%$ \\
\hline B3 & & & 0 & $0.0 \%$ \\
\hline B4 & & & 0 & $0.0 \%$ \\
\hline B5 & 109.6 & 109.9 & 0.3 & $0.4 \%$ \\
\hline B6 & 111.5 & 110.5 & 0 & $0.0 \%$ \\
\hline DS in-1 & & & 0 & \multirow[b]{2}{*}{$0.0 \%$} \\
\hline DS in-2 & & & 0 & \\
\hline DS out-1 & 65.5 & 69.4 & 3.9 & \multirow[b]{4}{*}{$5.5 \%$} \\
\hline DS out-2 & & & 0 & \\
\hline DS out-3 & & & 0 & \\
\hline DS out-4 & & & 0 & \\
\hline Other & & & 0 & $0.0 \%$ \\
\hline
\end{tabular}

\begin{tabular}{|c|c|}
\hline Test Date & \\
\hline \multicolumn{2}{|c|}{ Total Input 70.8} \\
\hline \multicolumn{2}{|c|}{ Total Collected 37.6} \\
\hline \multicolumn{2}{|c|}{ "Difference -33.2} \\
\hline & \\
\hline
\end{tabular}

\begin{tabular}{|lr|}
\hline \multicolumn{2}{|c|}{ Evaporation } \\
Evap Pan Tare Wt, g & 66.7 \\
Evaporation, g & 1.1 \\
\hline \% Evaporation & $\mathbf{3 . 7 3 \%}$ \\
\hline
\end{tabular}

\begin{tabular}{|lr|}
\hline \multicolumn{2}{|c|}{ Mass Flow Rate } \\
Test Duration, min & 301.1 \\
Flow Rate, g/min & 0.24 \\
\hline
\end{tabular}

\begin{tabular}{|lr|}
\hline \multicolumn{2}{|c|}{ Reference Towel Mass } \\
Initial Mass $(\mathrm{g})$ & 7.8 \\
Final Mass $(\mathrm{g})$ & 8.2 \\
\hline \hline Difference $(\mathrm{g})$ & 0.4 \\
\hline
\end{tabular}

\begin{tabular}{|c|c|c|c|c|}
\hline \multicolumn{5}{|c|}{ Test Chamber Environment } \\
\hline Initial Conditions & $\mathrm{RH}(\%)$ & Temperature $\left({ }^{\circ} \mathrm{C}\right)$ & & Atm. P. (mbar) \\
\hline 1-HUM/TMP-BDST & 87.1 & 27.4 & P-IN-BDST & 846 \\
\hline 2-HUM/TMP-BDST & 87.1 & 27.4 & & \\
\hline Final Conditions & $\mathrm{RH}(\%)$ & Temperature $\left({ }^{\circ} \mathrm{C}\right)$ & & Atm. P. (mbar) \\
\hline 1-HUM/TMP-BDST & 99 & 29.9 & P-IN-BDST & 874 \\
\hline 2-HUM/TMP-BDST & 94.2 & 29.5 & & \\
\hline
\end{tabular}


Bounding Flow Test Summary and Mass Balance - Rough DS Surface

Test: High flow rate, $54 \mathrm{~cm}$ left of DS center, 16.5

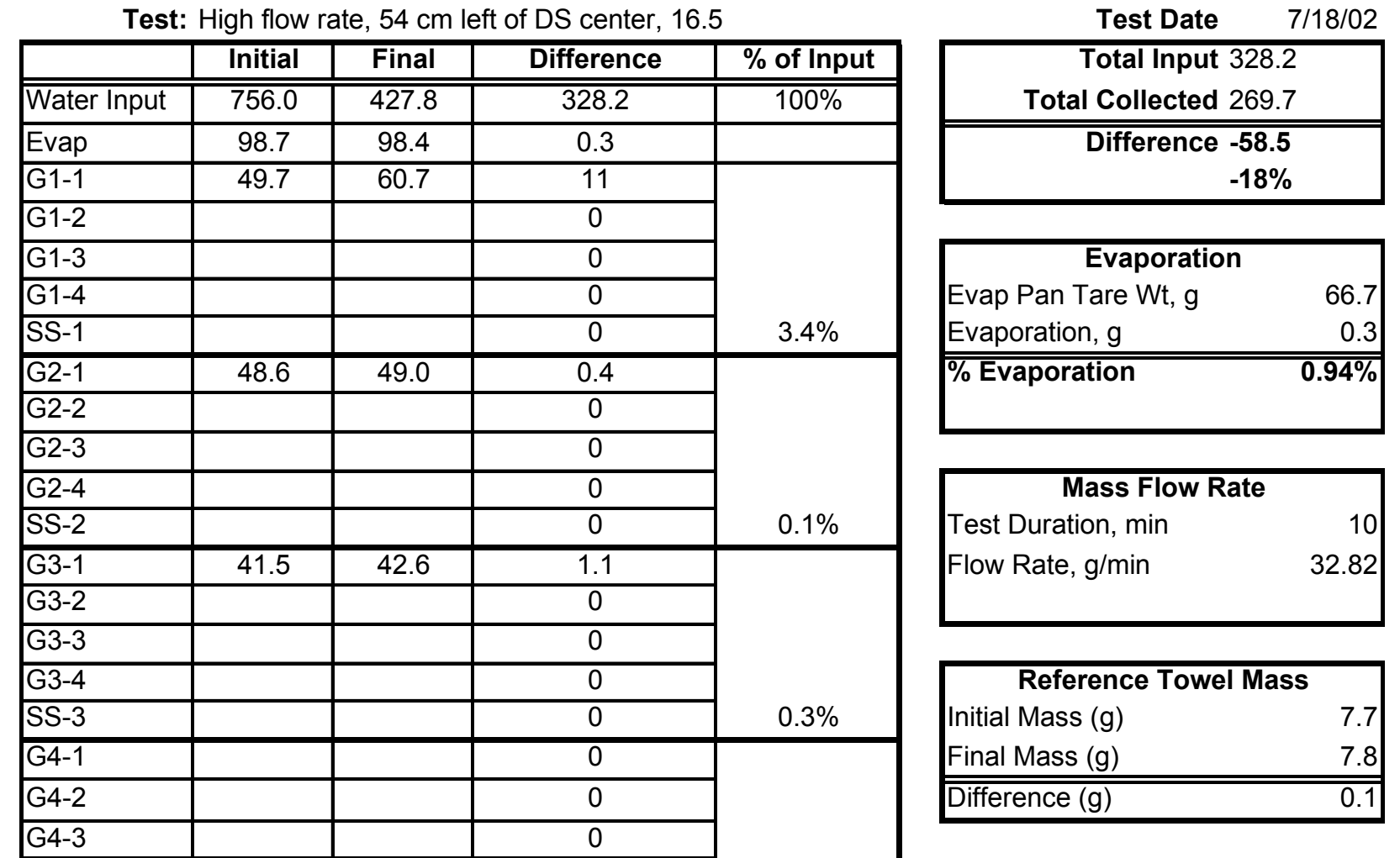

\begin{tabular}{|c|c|c|c|c|}
\hline & & & & \multirow[b]{3}{*}{$0.0 \%$} \\
\hline G4-4 & & & 0 & \\
\hline SS-4 & & & 0 & \\
\hline B1 & & & 0 & $0.0 \%$ \\
\hline B2 & & & 0 & $0.0 \%$ \\
\hline B3 & & & 0 & $0.0 \%$ \\
\hline B4 & 107.7 & 305.6 & 197.9 & $60.3 \%$ \\
\hline B5 & & & 0 & $0.0 \%$ \\
\hline B6 & & & 0 & $0.0 \%$ \\
\hline DS in-1 & & & 0 & \multirow[b]{2}{*}{$0.0 \%$} \\
\hline DS in-2 & & & 0 & \\
\hline DS out-1 & 71.6 & 130.9 & 59.3 & \multirow[b]{4}{*}{$18.1 \%$} \\
\hline DS out-2 & & & 0 & \\
\hline DS out-3 & & & 0 & \\
\hline DS out-4 & & & 0 & \\
\hline Other & & & 0 & $0.0 \%$ \\
\hline
\end{tabular}

\begin{tabular}{|lcccc|}
\hline \multicolumn{4}{l}{ Test Chamber Environment } \\
\hline Initial Conditions & $\mathrm{RH}(\%)$ & Temperature $\left({ }^{\circ} \mathrm{C}\right)$ & Atm. P. (mbar) \\
1-HUM/TMP-BDST & 84.8 & 24 & P-IN-BDST & 859 \\
2-HUM/TMP-BDST & 85 & 24 & & \\
\hline Final Conditions & $\mathrm{RH}(\%)$ & Temperature $\left({ }^{\circ} \mathrm{C}\right)$ & & Atm. P. (mbar) \\
1-HUM/TMP-BDST & 89.6 & 24.4 & P-IN-BDST & 850 \\
2-HUM/TMP-BDST & 89 & 24.3 & & \\
\hline
\end{tabular}


Bounding Flow Test Summary and Mass Balance - Rough DS Surface

Test: Low flow rate, $54 \mathrm{~cm}$ left of DS Center, 16.5

\begin{tabular}{|c|c|c|c|c|}
\hline & Initial & Final & Difference & $\%$ of Input \\
\hline Water Input & 627.0 & 532.6 & 94.4 & $100 \%$ \\
\hline Evap & 98.2 & 97.7 & 0.5 & \\
\hline G1-1 & 42.4 & 42.6 & 0.2 & \multirow[b]{5}{*}{$0.2 \%$} \\
\hline G1-2 & & & 0 & \\
\hline G1-3 & & & 0 & \\
\hline G1-4 & & & 0 & \\
\hline SS-1 & & & 0 & \\
\hline G2-1 & 41.2 & 41.2 & 0 & \multirow[b]{5}{*}{$0.0 \%$} \\
\hline G2-2 & & & 0 & \\
\hline G2-3 & & & 0 & \\
\hline G2-4 & & & 0 & \\
\hline SS-2 & & & 0 & \\
\hline G3-1 & 41.6 & 41.6 & 0 & \multirow[b]{5}{*}{$0.0 \%$} \\
\hline G3-2 & & & 0 & \\
\hline G3-3 & & & 0 & \\
\hline G3-4 & & & 0 & \\
\hline SS-3 & & & 0 & \\
\hline G4-1 & & & 0 & \multirow[b]{5}{*}{$0.0 \%$} \\
\hline G4-2 & & & 0 & \\
\hline G4-3 & & & 0 & \\
\hline G4-4 & & & 0 & \\
\hline SS-4 & & & 0 & \\
\hline $\mathrm{B1}$ & & & 0 & $0.0 \%$ \\
\hline B2 & & & 0 & $0.0 \%$ \\
\hline B3 & & & 0 & $0.0 \%$ \\
\hline B4 & 107.3 & 164.5 & 57.2 & $60.6 \%$ \\
\hline B5 & & & 0 & $0.0 \%$ \\
\hline B6 & 102.2 & 102.3 & 0.1 & $0.1 \%$ \\
\hline DS in-1 & & & 0 & \multirow[b]{2}{*}{$0.0 \%$} \\
\hline DS in-2 & & & 0 & \\
\hline DS out-1 & 56.7 & 59.1 & 2.4 & \multirow[b]{4}{*}{$2.5 \%$} \\
\hline DS out-2 & & & 0 & \\
\hline DS out-3 & & & 0 & \\
\hline DS out-4 & & & 0 & \\
\hline Other & & & 0 & $0.0 \%$ \\
\hline
\end{tabular}

Test Date $\quad 7 / 18 / 02$

\begin{tabular}{|r|}
\hline Total Input 94.4 \\
Total Collected 59.9 \\
\hline \hline Difference $\mathbf{- 3 4 . 5}$ \\
$\mathbf{- 3 7 \%}$ \\
\hline
\end{tabular}

\begin{tabular}{|lr|}
\hline \multicolumn{2}{|c|}{ Evaporation } \\
Evap Pan Tare Wt, g & 66.7 \\
Evaporation, g & 0.5 \\
\hline \% Evaporation & $\mathbf{1 . 5 9 \%}$ \\
\hline
\end{tabular}

\begin{tabular}{|lr|}
\hline \multicolumn{2}{|c|}{ Mass Flow Rate } \\
Test Duration, min & 300 \\
Flow Rate, g/min & 0.31 \\
\hline
\end{tabular}

\begin{tabular}{|lr|}
\hline \multicolumn{2}{|c|}{ Reference Towel Mass } \\
Initial Mass $(\mathrm{g})$ & 7.7 \\
Final Mass $(\mathrm{g})$ & 8.7 \\
\hline \hline Difference $(\mathrm{g})$ & 1.0 \\
\hline
\end{tabular}

\begin{tabular}{|lcccc|}
\hline \multicolumn{5}{ll}{ Test Chamber Environment } \\
\hline Initial Conditions & $\mathrm{RH}(\%)$ & Temperature $\left({ }^{\circ} \mathrm{C}\right)$ & Atm. P. (mbar) \\
1-HUM/TMP-BDST & 86.7 & 23.8 & P-IN-BDST & 858 \\
2-HUM/TMP-BDST & 84.3 & 24.4 & & \\
\hline Final Conditions & $\mathrm{RH}(\%)$ & Temperature $\left({ }^{\circ} \mathrm{C}\right)$ & Atm. P. (mbar) \\
1-HUM/TMP-BDST & 108.5 & 27.7 & P-IN-BDST & 852 \\
2-HUM/TMP-BDST & 90.7 & 28.1 & & \\
\hline
\end{tabular}


Bounding Flow Test Summary and Mass Balance - Rough DS Surface

Test: High flow rate, $27 \mathrm{~cm}$ left of DS Center, 16.5

Test Date $\quad 7 / 18 / 02$

\begin{tabular}{|l|c|c|c|c|}
\hline & Initial & Final & Difference & \% of Input \\
\hline \hline Water Input & 790.8 & 468.7 & 322.1 & $100 \%$ \\
\hline Evap & 99.7 & 99.0 & 0.7 & \\
\hline G1-1 & 64.5 & 244.2 & 179.7 &
\end{tabular}

Total Input 322.1

Total Collected 264

Difference $\mathbf{- 5 8 . 1}$

$-18 \%$

\begin{tabular}{|c|c|c|c|c|}
\hline G1-2 & 63.0 & 89.9 & 26.9 & \multirow[b]{4}{*}{$64.1 \%$} \\
\hline G1-3 & & & 0 & \\
\hline G1-4 & & & 0 & \\
\hline SS-1 & & & 0 & \\
\hline G2-1 & 41.1 & 41.1 & 0 & \\
\hline G2-2 & & & 0 & \\
\hline
\end{tabular}

G2-3

G2-4

SS-2

0

G3-1

G3-2

G3-3

G3-4

SS-3

0

G4-1

G4-2

G4-3

G4-4

SS-4

B1

B2

B3

\begin{tabular}{l}
$\mathrm{B} 4$ \\
\hline $\mathrm{B} 5$ \\
\hline
\end{tabular}

$\mathrm{B} 5$

\begin{tabular}{|l|}
101.6 \\
\hline B6
\end{tabular}

\begin{tabular}{|c|c|c|c|c|}
\hline B6 & 110.2 & 110.5 & 0.3 & $0.1 \%$ \\
\hline DS in-1 & & & 0 & \multirow[b]{2}{*}{$0.0 \%$} \\
\hline DS in-2 & & & 0 & \\
\hline DS out-1 & 71.9 & 124.8 & 52.9 & \multirow[b]{4}{*}{$16.4 \%$} \\
\hline DS out-2 & & & 0 & \\
\hline DS out-3 & & & 0 & \\
\hline DS out-4 & & & 0 & \\
\hline Other & & & 0 & $0.0 \%$ \\
\hline
\end{tabular}

Test Chamber Environment

\begin{tabular}{lcccl}
\hline Initial Conditions & $\mathrm{RH}(\%)$ & Temperature $\left({ }^{\circ} \mathrm{C}\right)$ & \\
1-HUM/TMP-BDST & 88.1 & 24.2 & P-IN-BDST \\
2-HUM/TMP-BDST & 85 & 24.5 & \\
\hline Final Conditions & $\mathrm{RH}(\%)$ & Temperature $\left({ }^{\circ} \mathrm{C}\right)$ & \\
1-HUM/TMP-BDST & 88.5 & 24.4 & P-IN-BDST \\
2-HUM/TMP-BDST & 88.7 & 24.4 & \\
\hline
\end{tabular}

Atm. P. (mbar)

860

Atm. P. (mbar)

862

\section{Evaporation}

Evap Pan Tare Wt, g

Evaporation, $\mathrm{g}$

\% Evaporation

$2.12 \%$

Mass Flow Rate

Test Duration, min

Flow Rate, g/min

10

32.21

\begin{tabular}{|lr|}
\hline \multicolumn{2}{|c|}{ Reference Towel Mass } \\
Initial Mass $(\mathrm{g})$ & 7.8 \\
Final Mass $(\mathrm{g})$ & 7.8 \\
\hline \hline Difference $(\mathrm{g})$ & 0.0 \\
\hline
\end{tabular}


Bounding Flow Test Summary and Mass Balance - Rough DS Surface

Test: Low flow rate, $27 \mathrm{~cm}$ left of DS Center, 16.5

\begin{tabular}{|c|c|c|c|c|c|c|}
\hline \multicolumn{5}{|l|}{ Test } & \multicolumn{2}{|c|}{ Test Date $\quad 7 / 24 / 02$} \\
\hline & Initial & Final & Difference & $\%$ of Input & \multicolumn{2}{|c|}{ Total Input 116.0} \\
\hline Water Input & 795.6 & 679.6 & 116 & $100 \%$ & \multicolumn{2}{|c|}{ Total Collected 95} \\
\hline Evap & 80.8 & 80.5 & 0.33 & & \multirow{2}{*}{\multicolumn{2}{|c|}{ 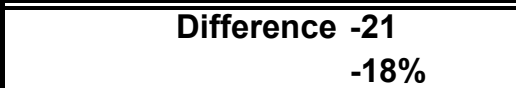 }} \\
\hline G1-1 & 66.4 & 156.2 & 89.8 & \multirow[b]{5}{*}{$77.5 \%$} & & \\
\hline G1-2 & & & 0 & & & \\
\hline G1-3 & & & 0 & & \multicolumn{2}{|c|}{ Evaporation } \\
\hline G1-4 & & & 0 & & \multirow{2}{*}{$\begin{array}{l}\text { Evap Pan Tare Wt, g } \\
\text { Evaporation, g }\end{array}$} & 66.7 \\
\hline SS-1 & 42.0 & 42.1 & 0.1 & & & 0.33 \\
\hline G2-1 & & & 0 & \multirow[b]{5}{*}{$0.0 \%$} & \multirow[t]{2}{*}{ \% Evaporation } & \multirow[t]{2}{*}{$2.34 \%$} \\
\hline G2-2 & & & 0 & & & \\
\hline G2-3 & & & 0 & & & \\
\hline G2-4 & & & 0 & & \multicolumn{2}{|c|}{ Mass Flow Rate } \\
\hline SS-2 & & & 0 & & \multirow{3}{*}{$\begin{array}{l}\text { Test Duration, min } \\
\text { Flow Rate, g/min }\end{array}$} & \multirow{3}{*}{$\begin{array}{r}300.2 \\
0.39\end{array}$} \\
\hline$\overline{G 3-1}$ & & & 0 & \multirow[b]{5}{*}{$0.0 \%$} & & \\
\hline G3-2 & & & 0 & & & \\
\hline G3-3 & & & 0 & & & \\
\hline G3-4 & & & 0 & & \multicolumn{2}{|c|}{ Reference Towel Mass } \\
\hline SS-3 & 40.5 & 40.5 & 0 & & \multirow{2}{*}{$\begin{array}{l}\text { Initial Mass }(\mathrm{g}) \\
\text { Final Mass }(\mathrm{g})\end{array}$} & 7.7 \\
\hline G4-1 & & & 0 & & & 8.5 \\
\hline G4-2 & & & 0 & & Difference (g) & 0.8 \\
\hline G4-3 & & & 0 & & & \\
\hline
\end{tabular}

\begin{tabular}{|c|c|c|c|c|}
\hline & & & & \multirow[b]{3}{*}{$0.0 \%$} \\
\hline G4-4 & & & 0 & \\
\hline SS-4 & & & 0 & \\
\hline B1 & & & 0 & $0.0 \%$ \\
\hline B2 & 107.0 & 107.8 & 0.8 & $0.7 \%$ \\
\hline B3 & & & 0 & $0.0 \%$ \\
\hline B4 & 107.5 & 107.9 & 0.4 & $0.3 \%$ \\
\hline B5 & 109.4 & 109.9 & 0.5 & $0.4 \%$ \\
\hline B6 & 110.1 & 110.4 & 0.3 & $0.3 \%$ \\
\hline DS in-1 & & & 0 & \multirow[b]{2}{*}{$0.0 \%$} \\
\hline DS in-2 & & & 0 & \\
\hline DS out- 1 & 63.8 & 66.9 & 3.1 & \multirow[b]{4}{*}{$2.7 \%$} \\
\hline DS out-2 & & & 0 & \\
\hline DS out-3 & & & 0 & \\
\hline DS out- 4 & & & 0 & \\
\hline Other & & & 0 & $0.0 \%$ \\
\hline
\end{tabular}

\begin{tabular}{|lcccc|}
\hline Test Chamber Environment & & & \\
\hline Initial Conditions & $\mathrm{RH}(\%)$ & Temperature $\left({ }^{\circ} \mathrm{C}\right)$ & Atm. P. (mbar) \\
1-HUM/TMP-BDST & 86.7 & 20.9 & P-IN-BDST & 843 \\
2-HUM/TMP-BDST & 92.4 & 20.4 & & \\
\hline Final Conditions & $\mathrm{RH}(\%)$ & Temperature $\left({ }^{\circ} \mathrm{C}\right)$ & & Atm. P. (mbar) \\
1-HUM/TMP-BDST & 96.3 & 28.1 & P-IN-BDST & 852 \\
2-HUM/TMP-BDST & 95 & 27.6 & & \\
\hline
\end{tabular}


Bounding Flow Test Summary and Mass Balance - Rough DS Surface

Test: High flow rate, $27 \mathrm{~cm}$ right of DS center, 16.5

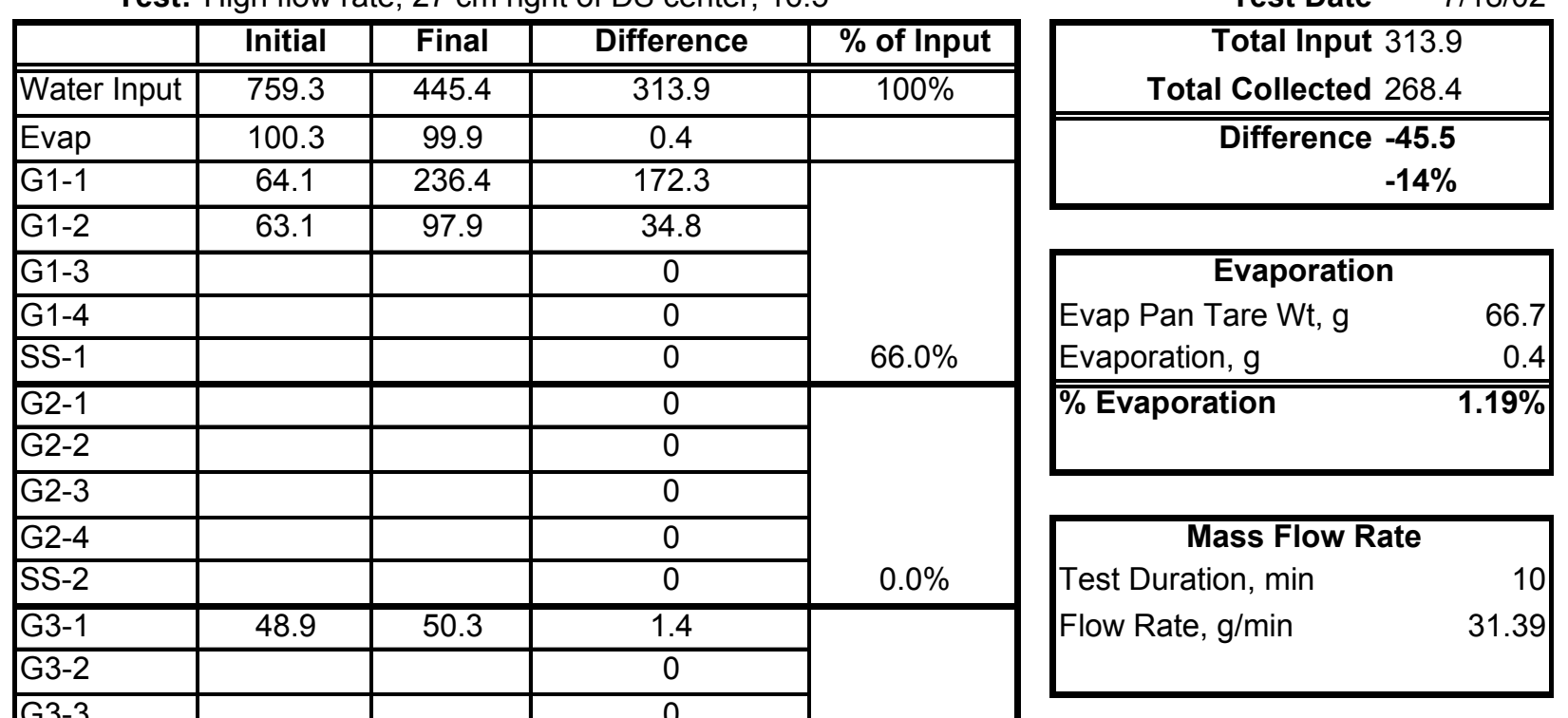

\begin{tabular}{|c|c|c|c|c|}
\hline G3-3 & & & 0 & \multirow[b]{3}{*}{$0.4 \%$} \\
\hline G3-4 & & & 0 & \\
\hline SS-3 & & & 0 & \\
\hline G4-1 & 41.0 & 41.2 & 0.2 & \multirow[b]{5}{*}{$0.1 \%$} \\
\hline G4-2 & & & 0 & \\
\hline G4-3 & & & 0 & \\
\hline G4-4 & & & 0 & \\
\hline SS-4 & & & 0 & \\
\hline B1 & & & 0 & $0.0 \%$ \\
\hline B2 & 106.8 & 107.6 & 0.8 & $0.3 \%$ \\
\hline B3 & & & 0 & $0.0 \%$ \\
\hline B4 & & & 0 & $0.0 \%$ \\
\hline B5 & 109.3 & 110.4 & 1.1 & $0.4 \%$ \\
\hline B6 & 8.4 & 8.6 & 0.2 & $0.1 \%$ \\
\hline DS in-1 & & & 0 & \multirow[b]{2}{*}{$0.0 \%$} \\
\hline DS in-2 & & & 0 & \\
\hline DS out-1 & 64.0 & 121.6 & 57.6 & \multirow{5}{*}{$18.3 \%$} \\
\hline DS out-2 & & & 0 & \\
\hline DS out-3 & & & 0 & \\
\hline DS out- 4 & & & 0 & \\
\hline Other & & & 0 & \\
\hline
\end{tabular}

\begin{tabular}{|lr|}
\hline \multicolumn{2}{|c|}{ Reference Towel Mass } \\
Initial Mass (g) & 7.6 \\
Final Mass (g) & 7.9 \\
\hline \hline Difference (g) & 0.3 \\
\hline
\end{tabular}

\begin{tabular}{|lcccc|}
\hline \multicolumn{6}{l}{ Test Chamber Environment } \\
\hline Initial Conditions & $\mathrm{RH}(\%)$ & Temperature $\left({ }^{\circ} \mathrm{C}\right)$ & & Atm. P. (mbar) \\
1-HUM/TMP-BDST & 83.6 & 24.7 & P-IN-BDST & 875 \\
2-HUM/TMP-BDST & 85.7 & 24.7 & & \\
\hline Final Conditions & $\mathrm{RH}(\%)$ & Temperature $\left({ }^{\circ} \mathrm{C}\right)$ & & Atm. P. (mbar) \\
1-HUM/TMP-BDST & 91.7 & 25.5 & P-IN-BDST & 861 \\
2-HUM/TMP-BDST & 92.8 & 25.5 & & \\
\hline
\end{tabular}

SN-M\&O-SCl-043-V2, pages 186-187 
Bounding Flow Test Summary and Mass Balance - Rough DS Surface

Test: Low flow rate, $27 \mathrm{~cm}$ right of DS Center, 16.5

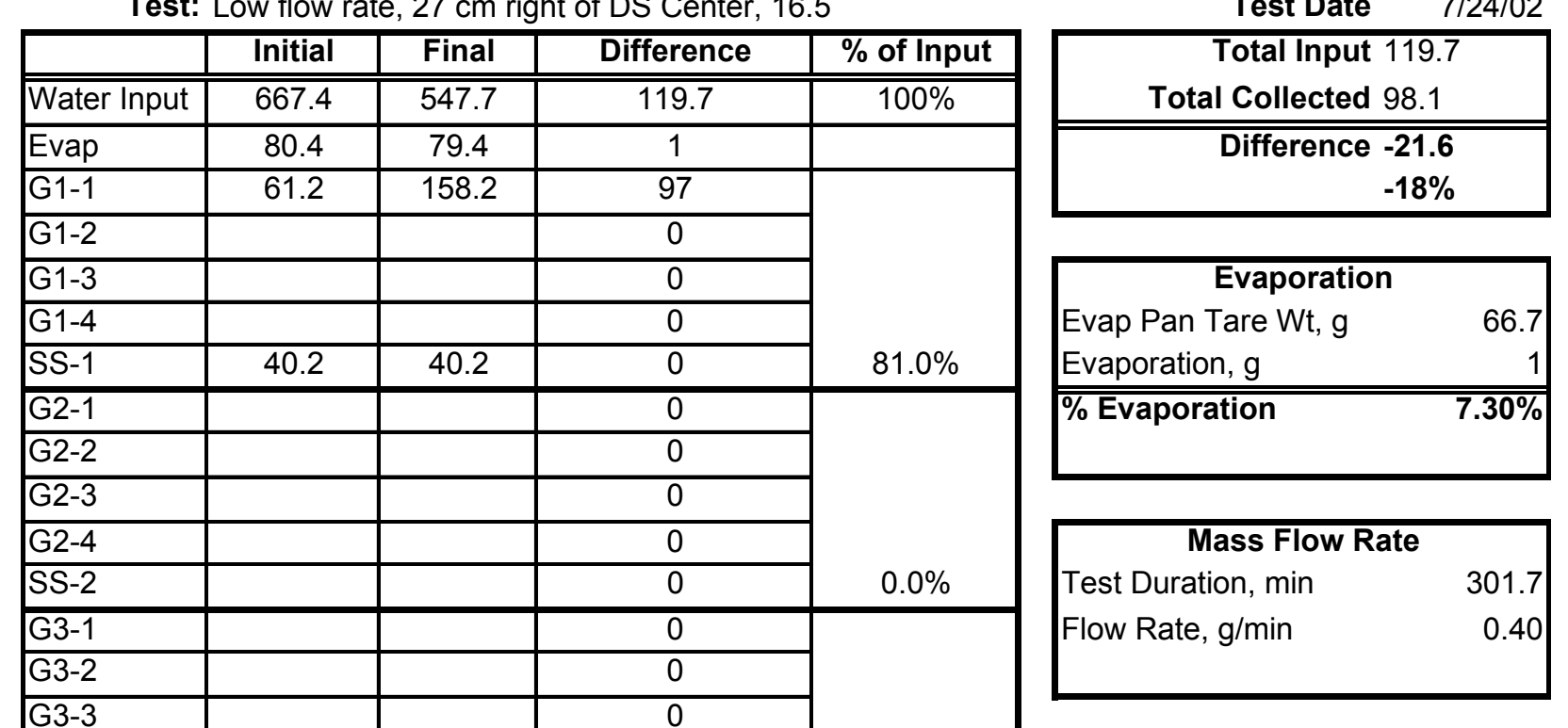

\begin{tabular}{|c|c|c|c|c|}
\hline G3-3 & & & 0 & \multirow[b]{3}{*}{$0.0 \%$} \\
\hline G3-4 & & & 0 & \\
\hline SS-3 & 40.9 & 40.9 & 0 & \\
\hline G4-1 & & & 0 & \multirow[b]{5}{*}{$0.0 \%$} \\
\hline G4-2 & & & 0 & \\
\hline G4-3 & & & 0 & \\
\hline G4-4 & & & 0 & \\
\hline SS-4 & & & 0 & \\
\hline B1 & & & 0 & $0.0 \%$ \\
\hline$\overline{B 2}$ & 107.7 & 107.8 & 0.1 & $0.1 \%$ \\
\hline B3 & & & 0 & $0.0 \%$ \\
\hline B4 & & & 0 & $0.0 \%$ \\
\hline B5 & 109.9 & 110.0 & 0.1 & $0.1 \%$ \\
\hline B6 & 110.4 & 110.4 & 0 & $0.0 \%$ \\
\hline DS in-1 & & & 0 & \multirow[b]{2}{*}{$0.0 \%$} \\
\hline DS in-2 & & & 0 & \\
\hline DS out-1 & 62.8 & 63.7 & 0.9 & \multirow[b]{4}{*}{$0.8 \%$} \\
\hline$\overline{D S}$ out- 2 & & & 0 & \\
\hline DS out-3 & & & 0 & \\
\hline DS out-4 & & & 0 & \\
\hline Other & & & 0 & $0.0 \%$ \\
\hline
\end{tabular}

\begin{tabular}{|lr|}
\hline \multicolumn{2}{|c|}{ Reference Towel Mass } \\
Initial Mass $(\mathrm{g})$ & 8.1 \\
Final Mass $(\mathrm{g})$ & 8.2 \\
\hline \hline Difference $(\mathrm{g})$ & 0.1 \\
\hline
\end{tabular}

\begin{tabular}{|lcclc|}
\hline Test Chamber Environment & & & \\
\hline Initial Conditions & $\mathrm{RH}(\%)$ & Temperature $\left({ }^{\circ} \mathrm{C}\right)$ & Atm. P. (mbar) \\
1-HUM/TMP-BDST & 95.2 & 28.3 & P-IN-BDST & 862 \\
2-HUM/TMP-BDST & 94.5 & 27.7 & & \\
\hline Final Conditions & $\mathrm{RH}(\%)$ & Temperature $\left({ }^{\circ} \mathrm{C}\right)$ & & Atm. P. (mbar) \\
1-HUM/TMP-BDST & 98 & 29.7 & P-IN-BDST & 868 \\
2-HUM/TMP-BDST & 94.9 & 29.2 & & \\
\hline
\end{tabular}


Bounding Flow Test Summary and Mass Balance - Rough DS Surface

Test: High flow rate, $54 \mathrm{~cm}$ left of DS center, 33

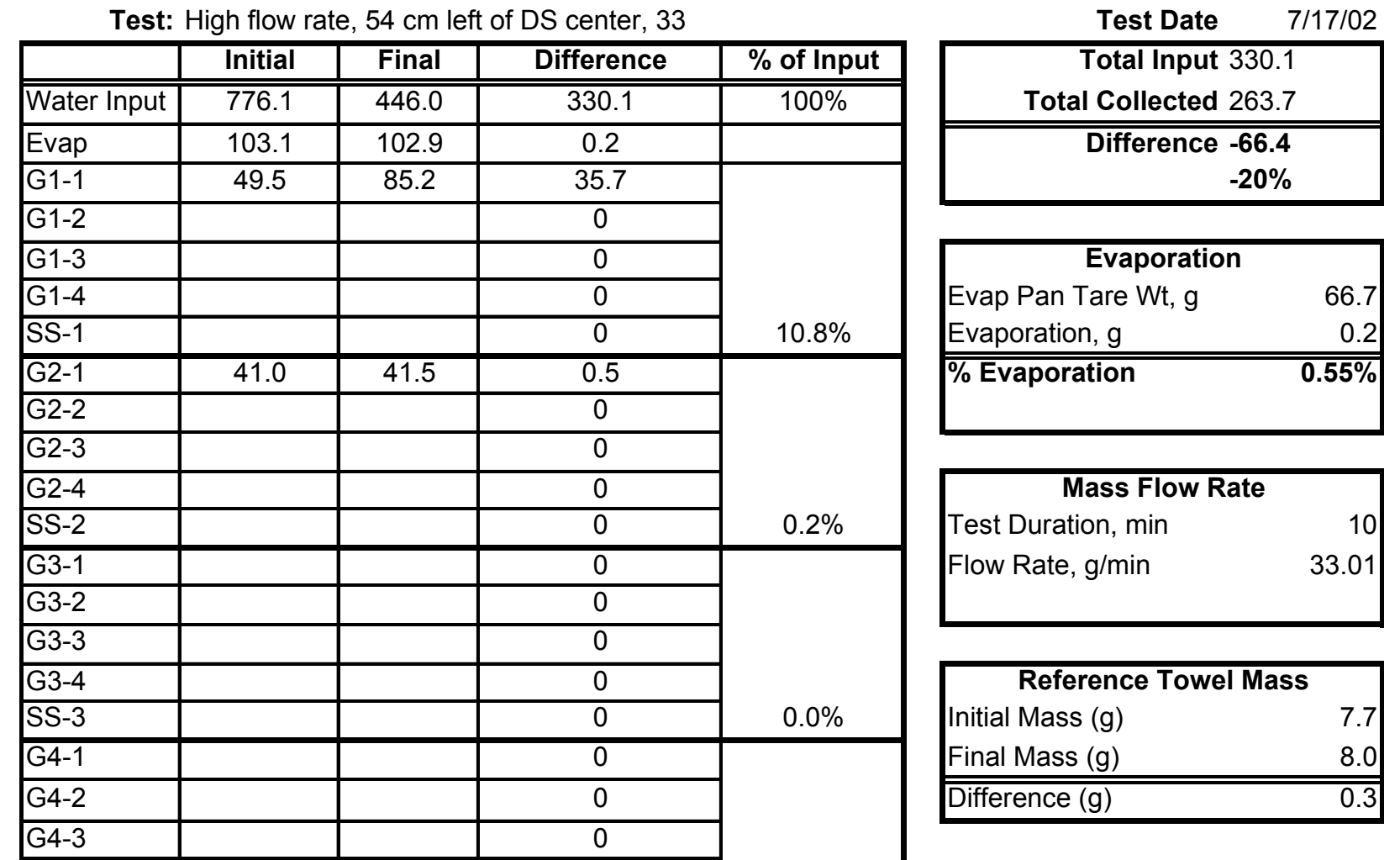

\begin{tabular}{|c|c|c|c|c|}
\hline 4 & & & 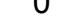 & \multirow[b]{3}{*}{$0.0 \%$} \\
\hline G4-4 & & & 0 & \\
\hline SS-4 & & & 0 & \\
\hline B1 & & & 0 & $0.0 \%$ \\
\hline B2 & & & 0 & $0.0 \%$ \\
\hline B3 & & & 0 & $0.0 \%$ \\
\hline B4 & 106.0 & 311.0 & 205 & $62.1 \%$ \\
\hline B5 & & & 0 & $0.0 \%$ \\
\hline B6 & & & 0 & $0.0 \%$ \\
\hline DS in-1 & & & 0 & \multirow[b]{2}{*}{$0.0 \%$} \\
\hline DS in-2 & & & 0 & \\
\hline DS out-1 & 49.1 & 71.6 & 22.5 & \multirow[b]{4}{*}{$6.8 \%$} \\
\hline DS out- 2 & & & 0 & \\
\hline DS out-3 & & & 0 & \\
\hline DS out-4 & & & 0 & \\
\hline Other & & & 0 & $0.0 \%$ \\
\hline
\end{tabular}

\begin{tabular}{|lcccc|}
\hline \multicolumn{5}{l}{ Test Chamber Environment } \\
\hline Initial Conditions & $\mathrm{RH}(\%)$ & Temperature $\left({ }^{\circ} \mathrm{C}\right)$ & \\
1-HUM/TMP-BDST & 87.2 & 24.7 & P-IN-BDST & Atm. P. (mbar) \\
2-HUM/TMP-BDST & 89.3 & 24.5 & & 856 \\
\hline Final Conditions & $\mathrm{RH}(\%)$ & Temperature $\left({ }^{\circ} \mathrm{C}\right)$ & & Atm. P. (mbar) \\
1-HUM/TMP-BDST & 89.9 & 25.1 & P-IN-BDST & 860 \\
2-HUM/TMP-BDST & 90.9 & 24.9 & & \\
\hline
\end{tabular}


Bounding Flow Test Summary and Mass Balance - Rough DS Surface

Test: Low flow rate, $54 \mathrm{~cm}$ left of DS center, 33

\begin{tabular}{|c|c|c|c|c|c|c|}
\hline \multicolumn{5}{|c|}{ Test: Low flow rate, $54 \mathrm{~cm}$ left of DS center, 33} & \multicolumn{2}{|c|}{ Test Date } \\
\hline & Initial & Final & Difference & $\%$ of Input & \multirow{2}{*}{\multicolumn{2}{|c|}{$\begin{array}{r}\text { Total Input } 123.1 \\
\text { Total Collected } 54.5 \\
\end{array}$}} \\
\hline Water Input & 682.2 & 559.1 & 123.1 & $100 \%$ & & \\
\hline Evap & 101.3 & 100.3 & 1 & & \multirow{2}{*}{\multicolumn{2}{|c|}{$\begin{array}{r}\text { Difference }-68.6 \\
-56 \%\end{array}$}} \\
\hline G1-1 & 42.2 & 42.4 & 0.2 & \multirow[b]{5}{*}{$0.2 \%$} & & \\
\hline G1-2 & & & 0 & & & \\
\hline G1-3 & & & 0 & & \multicolumn{2}{|c|}{ Evaporation } \\
\hline G1-4 & & & 0 & & \multirow{2}{*}{\multicolumn{2}{|c|}{$\begin{array}{lr}\text { Evap Pan Tare Wt, g } & 66.7 \\
\text { Evaporation, g } & 1\end{array}$}} \\
\hline SS-1 & & & 0 & & & \\
\hline G2-1 & 41.1 & 41.1 & 0 & \multirow[b]{5}{*}{$0.0 \%$} & \multirow{2}{*}{\multicolumn{2}{|c|}{ \% Evaporation }} \\
\hline G2-2 & & & 0 & & & \\
\hline G2-3 & & & 0 & & & \\
\hline G2-4 & & & 0 & & \multicolumn{2}{|c|}{ Mass Flow Rate } \\
\hline SS-2 & & & 0 & & \multirow{3}{*}{$\begin{array}{l}\text { Test Duration, } \min \\
\text { Flow Rate, g/min }\end{array}$} & \multirow{3}{*}{$\begin{array}{r}300 \\
0.41\end{array}$} \\
\hline G3-1 & & & 0 & \multirow[b]{5}{*}{$0.0 \%$} & & \\
\hline G3-2 & & & 0 & & & \\
\hline G3-3 & & & 0 & & & \\
\hline G3-4 & & & 0 & & \multicolumn{2}{|c|}{ Reference Towel Mass } \\
\hline SS-3 & & & 0 & & \multirow{2}{*}{$\begin{array}{l}\text { Initial Mass }(\mathrm{g}) \\
\text { Final Mass }(\mathrm{g})\end{array}$} & 8.0 \\
\hline G4-1 & & & 0 & & & 8.3 \\
\hline G4-2 & & & 0 & & \multirow[t]{2}{*}{ Difference $(\mathrm{g})$} & 0.3 \\
\hline G4-3 & & & 0 & & & \\
\hline
\end{tabular}

\begin{tabular}{|c|c|c|c|c|}
\hline & & & & \multirow[b]{3}{*}{$0.0 \%$} \\
\hline G4-4 & & & 0 & \\
\hline SS-4 & & & 0 & \\
\hline B1 & & & 0 & $0.0 \%$ \\
\hline B2 & & & 0 & $0.0 \%$ \\
\hline B3 & & & 0 & $0.0 \%$ \\
\hline B4 & 106.2 & 159.4 & 53.2 & $43.2 \%$ \\
\hline B5 & & & 0 & $0.0 \%$ \\
\hline B6 & & & 0 & $0.0 \%$ \\
\hline DS in-1 & & & 0 & \multirow[b]{2}{*}{$0.0 \%$} \\
\hline DS in-2 & & & 0 & \\
\hline DS out-1 & 41.4 & 42.5 & 1.1 & \multirow[b]{4}{*}{$0.9 \%$} \\
\hline DS out-2 & & & 0 & \\
\hline DS out-3 & & & 0 & \\
\hline DS out-4 & & & 0 & \\
\hline Other & & & 0 & $0.0 \%$ \\
\hline
\end{tabular}

\begin{tabular}{|lcccc|}
\hline \multicolumn{5}{l}{ Test Chamber Environment } \\
\hline Initial Conditions & $\mathrm{RH}(\%)$ & Temperature $\left({ }^{\circ} \mathrm{C}\right)$ & Atm. P. (mbar) \\
1-HUM/TMP-BDST & 89 & 27.2 & P-IN-BDST & 852 \\
2-HUM/TMP-BDST & 89.9 & 26.9 & & \\
\hline Final Conditions & $\mathrm{RH}(\%)$ & Temperature $\left({ }^{\circ} \mathrm{C}\right)$ & Atm. P. (mbar) \\
1-HUM/TMP-BDST & 97 & 27.7 & P-IN-BDST & 875 \\
2-HUM/TMP-BDST & 86.5 & 28.7 & & \\
\hline
\end{tabular}


Bounding Flow Test Summary and Mass Balance - Rough DS Surface

Test: High flow rate, $27 \mathrm{~cm}$ left of DS center, 33

\begin{tabular}{|c|c|c|c|c|c|c|}
\hline \multicolumn{5}{|c|}{ Test: High flov } & \multicolumn{2}{|c|}{ Test Date $\quad 7 / 17 / 02$} \\
\hline & Initial & Final & Difference & $\%$ of Input & \multirow{2}{*}{\multicolumn{2}{|c|}{$\begin{array}{r}\text { Total Input } 339.2 \\
\text { Total Collected } 252.1\end{array}$}} \\
\hline Water Input & $\begin{array}{ll}798.7 \\
\end{array}$ & $4 \overline{459.5}$ & $=339.2$ & $=100 \%$ & & \\
\hline Evap & 102.5 & 102.2 & 0.3 & & \multirow{2}{*}{\multicolumn{2}{|c|}{$\begin{array}{r}\text { Difference }-87.1 \\
-26 \%\end{array}$}} \\
\hline$\overline{\mathrm{G} 1-1}$ & 63.9 & 256.2 & 192.3 & \multirow[b]{5}{*}{$69.0 \%$} & & \\
\hline$\overline{G 1-2}$ & 62.5 & 104.3 & 41.8 & & \\
\hline$\overline{\mathrm{G} 1-3}$ & & & 0 & & \multicolumn{2}{|c|}{ Evaporation } \\
\hline G1-4 & & & 0 & & \multirow{2}{*}{$\begin{array}{l}\text { Evap Pan Tare Wt, g } \\
\text { Evaporation, g }\end{array}$} & 66.7 \\
\hline SS-1 & & & 0 & & & \\
\hline$\overline{\mathrm{G} 2-1}$ & & & 0 & \multirow[b]{5}{*}{$0.0 \%$} & \multirow{2}{*}{\multicolumn{2}{|c|}{ \% Evaporation }} \\
\hline $\bar{G} 2-2$ & & & 0 & & & \\
\hline G2-3 & & & 0 & & \multirow{2}{*}{\multicolumn{2}{|c|}{ Mass Flow Rate }} \\
\hline $\bar{G} 2-4$ & & & 0 & & & \\
\hline SS-2 & & & 0 & & \multirow{3}{*}{$\begin{array}{l}\text { Mass Flow R } \\
\text { Test Duration, min } \\
\text { Flow Rate, } \mathrm{g} / \mathrm{min}\end{array}$} & 10 \\
\hline$\overline{\text { G3-1 }}$ & & & 0 & \multirow[b]{5}{*}{$0.0 \%$} & & 33.92 \\
\hline G3-2 & & & 0 & & & \\
\hline G3-3 & & & 0 & & & \\
\hline G3-4 & & & 0 & & \multicolumn{2}{|c|}{ Reference Towel Mass } \\
\hline SS-3 & & & 0 & & \multirow{2}{*}{$\begin{array}{l}\text { Initial Mass }(\mathrm{g}) \\
\text { Final Mass }(\mathrm{g})\end{array}$} & 7.8 \\
\hline$\overline{\mathrm{G} 4-1}$ & & & 0 & & & 7.8 \\
\hline G4-2 & & & 0 & & Difference (g) & 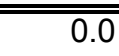 \\
\hline G4-3 & & & 0 & & & \\
\hline
\end{tabular}

\begin{tabular}{|c|c|c|c|c|}
\hline G4-4 & & & 0 & \\
\hline SS-4 & & & 0 & $0.0 \%$ \\
\hline B1 & & & 0 & $0.0 \%$ \\
\hline$\overline{B 2}$ & 107.0 & 107.0 & $\overline{0}$ & $0.0 \%$ \\
\hline B3 & & & 0 & $0.0 \%$ \\
\hline B4 & 107.5 & 107.6 & 0.1 & $0.0 \%$ \\
\hline B5 & & & 0 & $0.0 \%$ \\
\hline B6 & & & 0 & $0.0 \%$ \\
\hline DS in-1 & & & 0 & \multirow[b]{2}{*}{$0.0 \%$} \\
\hline DS in-2 & & & 0 & \\
\hline DS out-1 & 56.4 & 74.3 & 17.9 & \multirow[b]{4}{*}{$5.3 \%$} \\
\hline DS out- 2 & & & 0 & \\
\hline DS out-3 & & & 0 & \\
\hline$\overline{D S}$ out- 4 & & & 0 & \\
\hline Other & & & $\overline{0}$ & $0.0 \%$ \\
\hline
\end{tabular}

\begin{tabular}{|lcccc|}
\hline \multicolumn{7}{l}{ Test Chamber Environment } & & & \\
\hline Initial Conditions & $\mathrm{RH}(\%)$ & Temperature $\left({ }^{\circ} \mathrm{C}\right)$ & & Atm. P. (mbar) \\
1-HUM/TMP-BDST & 82 & 24.7 & P-IN-BDST & 849 \\
2-HUM/TMP-BDST & 83.3 & 25.4 & & \\
\hline Final Conditions & $\mathrm{RH}(\%)$ & Temperature $\left({ }^{\circ} \mathrm{C}\right)$ & & Atm. P. (mbar) \\
1-HUM/TMP-BDST & 84.8 & 24.6 & P-IN-BDST & 848 \\
2-HUM/TMP-BDST & 85.7 & 24.5 & & \\
\hline
\end{tabular}


Bounding Flow Test Summary and Mass Balance - Rough DS Surface

Test: Low flow rate, $27 \mathrm{~cm}$ left of DS center, 33

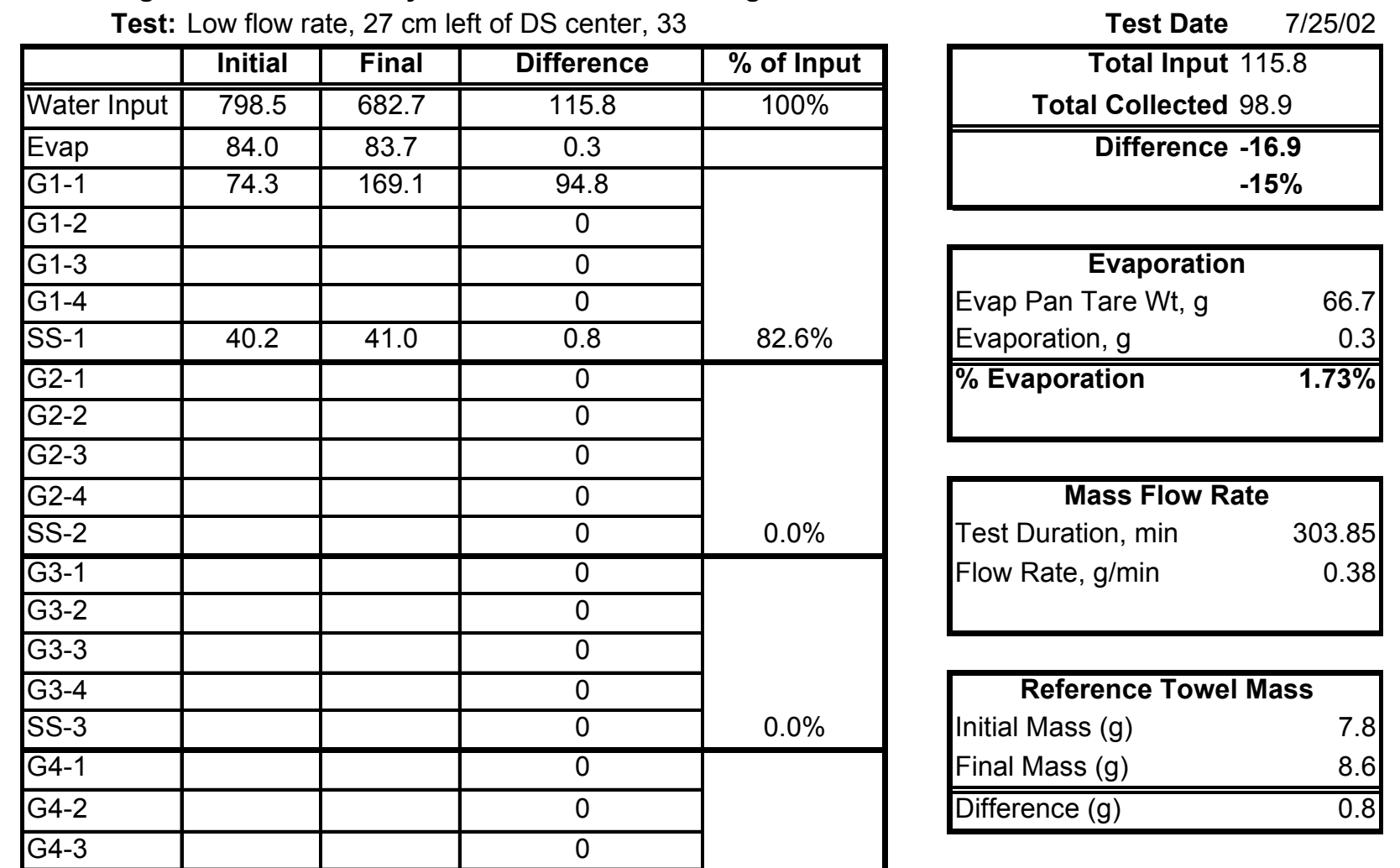

\begin{tabular}{|c|c|c|c|c|}
\hline G4-4 & & & 0 & \multirow[b]{2}{*}{$0.0 \%$} \\
\hline SS-4 & & & 0 & \\
\hline B1 & & & 0 & $0.0 \%$ \\
\hline B2 & 107.0 & 108.0 & 1 & $0.9 \%$ \\
\hline B3 & & & 0 & $0.0 \%$ \\
\hline B4 & 107.6 & 108.0 & 0.4 & $0.3 \%$ \\
\hline B5 & & & 0 & $0.0 \%$ \\
\hline B6 & & & 0 & $0.0 \%$ \\
\hline DS in-1 & & & 0 & \multirow[b]{2}{*}{$0.0 \%$} \\
\hline DS in-2 & & & 0 & \\
\hline DS out-1 & 69.4 & 71.3 & 1.9 & \multirow[b]{4}{*}{$1.6 \%$} \\
\hline DS out-2 & & & 0 & \\
\hline DS out-3 & & & 0 & \\
\hline DS out-4 & & & 0 & \\
\hline Other & & & 0 & $0.0 \%$ \\
\hline
\end{tabular}

$\begin{array}{lll}\text { Total Collected } & 98.9 & 85.4 \%\end{array}$

\begin{tabular}{|lcccc|}
\hline \multicolumn{5}{l}{ Test Chamber Environment } \\
\hline Initial Conditions & $\mathrm{RH}(\%)$ & Temperature $\left({ }^{\circ} \mathrm{C}\right)$ & Atm. P. (mbar) \\
1-HUM/TMP-BDST & 87.9 & 20.4 & P-IN-BDST & 850 \\
2-HUM/TMP-BDST & 92.7 & 20 & & \\
\hline Final Conditions & $\mathrm{RH}(\%)$ & Temperature $\left({ }^{\circ} \mathrm{C}\right)$ & Atm. P. (mbar) \\
1-HUM/TMP-BDST & 99.8 & 27.9 & P-IN-BDST & 850 \\
2-HUM/TMP-BDST & 95.2 & 27.5 & & \\
\hline
\end{tabular}


Bounding Flow Test Summary and Mass Balance - Rough DS Surface

Test: High flow rate, $27 \mathrm{~cm}$ right of DS center, 33

\begin{tabular}{|c|c|c|c|c|}
\hline & Initial & Final & Difference & $\%$ of Input \\
\hline Water Input & 803.2 & 472.9 & 330.3 & $100 \%$ \\
\hline Evap & 102.0 & 101.7 & 0.3 & \\
\hline $\mathrm{G} 1-1$ & 63.8 & 248.3 & 184.5 & \multirow[b]{5}{*}{$70.7 \%$} \\
\hline G1-2 & 62.7 & 111.6 & 48.9 & \\
\hline G1-3 & & & 0 & \\
\hline G1-4 & & & 0 & \\
\hline SS-1 & & & 0 & \\
\hline G2-1 & & & 0 & \multirow[b]{5}{*}{$0.0 \%$} \\
\hline G2-2 & & & 0 & \\
\hline G2-3 & & & 0 & \\
\hline G2-4 & & & 0 & \\
\hline SS-2 & & & 0 & \\
\hline G3-1 & & & 0 & \multirow[b]{5}{*}{$0.0 \%$} \\
\hline G3-2 & & & 0 & \\
\hline G3-3 & & & 0 & \\
\hline G3-4 & & & 0 & \\
\hline SS-3 & & & 0 & \\
\hline G4-1 & & & 0 & \multirow[b]{5}{*}{$0.0 \%$} \\
\hline G4-2 & & & 0 & \\
\hline G4-3 & & & 0 & \\
\hline G4-4 & & & 0 & \\
\hline SS-4 & & & 0 & \\
\hline B1 & & & 0 & $0.0 \%$ \\
\hline B2 & 106.9 & 107.0 & 0.1 & $0.0 \%$ \\
\hline B3 & & & 0 & $0.0 \%$ \\
\hline B4 & 107.5 & 107.6 & 0.1 & $0.0 \%$ \\
\hline B5 & 109.3 & 110.5 & 1.2 & $0.4 \%$ \\
\hline B6 & & & 0 & $0.0 \%$ \\
\hline DS in-1 & & & 0 & \multirow[b]{2}{*}{$0.0 \%$} \\
\hline DS in-2 & & & 0 & \\
\hline DS out-1 & 56.5 & 73.2 & 16.7 & \multirow[b]{4}{*}{$5.1 \%$} \\
\hline DS out-2 & & & 0 & \\
\hline DS out-3 & & & 0 & \\
\hline DS out-4 & & & 0 & \\
\hline Other & & & 0 & $0.0 \%$ \\
\hline
\end{tabular}

Test Date $\quad 7 / 17 / 02$

Total Input 330.3

Total Collected 251.5

Difference $\mathbf{- 7 8 . 8}$

$-24 \%$

\begin{tabular}{|lr|}
\hline \multicolumn{2}{|c|}{ Evaporation } \\
Evap Pan Tare Wt, g & 66.7 \\
Evaporation, g & 0.3 \\
\hline \% Evaporation & $\mathbf{0 . 8 5 \%}$ \\
\hline
\end{tabular}

\begin{tabular}{|lr|}
\hline \multicolumn{2}{|c|}{ Mass Flow Rate } \\
Test Duration, min & 10 \\
Flow Rate, g/min & 33.03 \\
\hline
\end{tabular}

Reference Towel Mass

Initial Mass (g) $\quad 7.7$

Final Mass $(\mathrm{g}) \quad 7.8$

Difference $(\mathrm{g}) \quad 0.1$

\begin{tabular}{|c|c|c|c|c|}
\hline Initial Conditions & $\mathrm{RH}(\%)$ & Temperature $\left({ }^{\circ} \mathrm{C}\right)$ & & Atm. P. (mbar) \\
\hline 1-HUM/TMP-BDST & 86.1 & 24.3 & P-IN-BDST & 854 \\
\hline 2-HUM/TMP-BDST & 86.1 & 24.6 & & \\
\hline Final Conditions & $\mathrm{RH}(\%)$ & Temperature $\left({ }^{\circ} \mathrm{C}\right)$ & & Atm. P. (mbar) \\
\hline 1-HUM/TMP-BDST & 88 & 24.8 & P-IN-BDST & 853 \\
\hline 2-HUM/TMP-BDST & 89.3 & 24.8 & & \\
\hline
\end{tabular}


Bounding Flow Test Summary and Mass Balance - Rough DS Surface

Test: Low flow rate, $27 \mathrm{~cm}$ right of DS center, 33

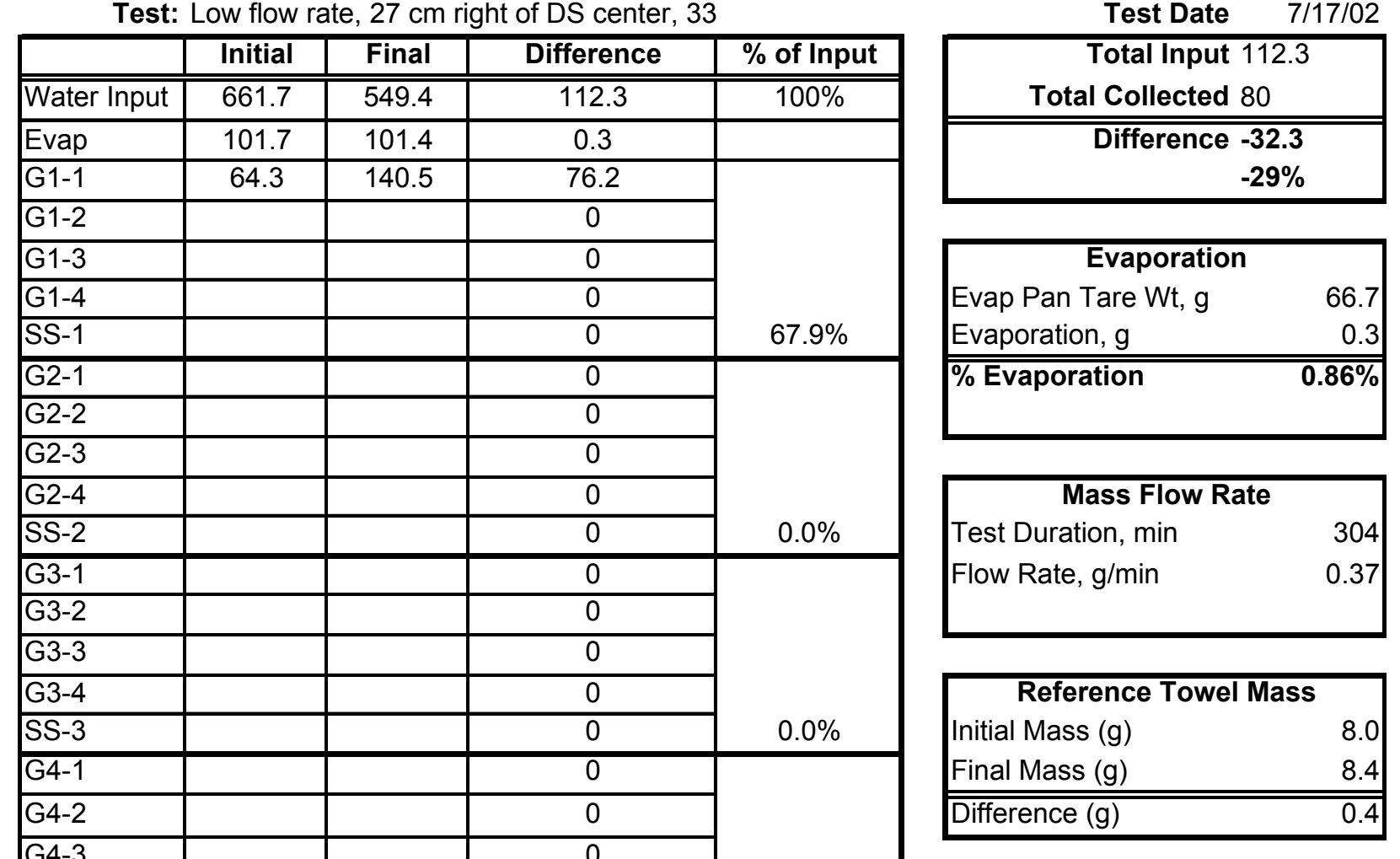

\begin{tabular}{|c|c|c|c|c|}
\hline G4-3 & & & 0 & \multirow[b]{3}{*}{$0.0 \%$} \\
\hline G4-4 & & & 0 & \\
\hline SS-4 & & & 0 & \\
\hline B1 & & & 0 & $0.0 \%$ \\
\hline B2 & & & 0 & $0.0 \%$ \\
\hline B3 & & & 0 & $0.0 \%$ \\
\hline B4 & & & 0 & $0.0 \%$ \\
\hline B5 & 109.7 & 110.5 & 0.8 & $0.7 \%$ \\
\hline B6 & & & 0 & $0.0 \%$ \\
\hline DS in-1 & & & 0 & \multirow[b]{2}{*}{$0.0 \%$} \\
\hline DS in-2 & & & 0 & \\
\hline DS out-1 & 49.2 & 52.2 & 3 & \multirow[b]{4}{*}{$2.7 \%$} \\
\hline DS out-2 & & & 0 & \\
\hline DS out-3 & & & 0 & \\
\hline DS out-4 & & & 0 & \\
\hline Other & & & 0 & $0.0 \%$ \\
\hline
\end{tabular}

\begin{tabular}{|c|c|c|c|c|}
\hline \multicolumn{5}{|c|}{ Test Chamber Environment } \\
\hline Initial Conditions & $\mathrm{RH}(\%)$ & Temperature $\left({ }^{\circ} \mathrm{C}\right)$ & & Atm. P. (mbar) \\
\hline 1-HUM/TMP-BDST & 91.6 & 25.9 & P-IN-BDST & 857 \\
\hline 2-HUM/TMP-BDST & 92.8 & 25.5 & & \\
\hline Final Conditions & $\mathrm{RH}(\%)$ & Temperature $\left({ }^{\circ} \mathrm{C}\right)$ & & Atm. P. (mbar) \\
\hline 1-HUM/TMP-BDST & 109.6 & 28.6 & P-IN-BDST & 863 \\
\hline 2-HUM/TMP-BDST & 95.1 & 28.4 & & \\
\hline
\end{tabular}




\section{APPENDIX C: \\ Rivulet Spread Data}

Test Description: Q (film) - Rivulet Flow

Patch 5, center, crown

Pages 116 and 117 of SN-M\&O-SCI-043-V1

\begin{tabular}{|l|c|c|c|}
\hline $\begin{array}{l}\text { Position relative to drip } \\
\text { impact }\end{array}$ & $\mathbf{3 3}^{\mathbf{0}}$ & $\begin{array}{c}\text { Transition } \\
\text { (Curved to vertical) }\end{array}$ & Vertical \\
\hline Maximum (cm) & -28.0 & -45.5 & -54.0 \\
\hline Minimum (cm) & 0 & 0 & 0 \\
\hline
\end{tabular}

Test Description: Q (film) - Rivulet Flow

Patch 5, $4 \mathrm{~cm}$ left of center, crown

Pages 118 and 119 of SN-M\&O-SCI-043-V1

\begin{tabular}{|l|c|c|c|}
\hline $\begin{array}{l}\text { Position relative to drip } \\
\text { impact }\end{array}$ & $\mathbf{3 3}^{\mathbf{0}}$ & $\begin{array}{c}\text { Transition } \\
\text { (Curved to vertical) }\end{array}$ & Vertical \\
\hline Maximum $(\mathrm{cm})$ & 21 & Not Recorded & Not Recorded \\
\hline Minimum $(\mathrm{cm})$ & Not Recorded & Not Recorded & Not Recorded \\
\hline
\end{tabular}

Test Description: Q (film) - Rivulet Flow

Patch 4, center, crown

Pages 120 and 121 of SN-M\&O-SCI-043-V1

\begin{tabular}{|l|c|c|c|}
\hline $\begin{array}{l}\text { Position relative to drip } \\
\text { impact }\end{array}$ & $\mathbf{3 3}^{\mathbf{0}}$ & $\begin{array}{c}\text { Transition } \\
\text { (Curved to vertical) }\end{array}$ & Vertical \\
\hline Right $(\mathrm{cm})$ & 26.5 & 19.0 & Not Visible at Test End \\
\hline Left $(\mathrm{cm})$ & 11.0 & 14.0 & 47.0 \\
\hline
\end{tabular}

Test Description: Q (film) - Rivulet Flow

Patch 4, $8 \mathrm{~cm}$ right of center, crown

Pages 122 and 123 of SN-M\&O-SCI-043-V1

\begin{tabular}{|l|c|c|c|}
\hline $\begin{array}{l}\text { Position relative to drip } \\
\text { impact }\end{array}$ & $\mathbf{3 3}^{\mathbf{0}}$ & $\begin{array}{c}\text { Transition } \\
\text { (Curved to vertical) }\end{array}$ & $\begin{array}{c}\text { Vertical } \\
\text { Top of Patch } 40.5 \mathrm{~cm} \text { from } \\
\text { Trans }\end{array}$ \\
\hline Right $(\mathrm{cm})$ & 28.5 & 36.5 & 45 \\
\hline Left $(\mathrm{cm})$ & 15.0 & 24 & 19 \\
\hline
\end{tabular}

Test Description: Q (film) - Rivulet Flow

Patch $4,8 \mathrm{~cm}$ right of center, $16.5^{\circ}$

Pages 124 and 125 of SN-M\&O-SCI-043-V1

\begin{tabular}{|l|c|c|c|}
\hline $\begin{array}{l}\text { Position relative to drip } \\
\text { impact }\end{array}$ & $\mathbf{3 3}^{\circ}$ & $\begin{array}{c}\text { Transition } \\
\text { (Curved to vertical) }\end{array}$ & $\begin{array}{c}\text { Vertical } \\
\text { (Top of patch on vertical) }\end{array}$ \\
\hline Right $($ East) $(\mathrm{cm})$ & 5.5 & 13.5 & 15 \\
\hline Left $($ West) $(\mathrm{cm})$ & 3.5 & N/A & N/A \\
\hline
\end{tabular}


Test Description: Q (film) - Rivulet Flow

Patch 2, center, $16.5^{\circ}$

Pages 126 and 127 of SN-M\&O-SCI-043-V1

\begin{tabular}{|l|c|c|c|}
\hline $\begin{array}{l}\text { Position relative to drip } \\
\text { impact }\end{array}$ & $\mathbf{3 3}^{\mathbf{0}}$ & $\begin{array}{c}\text { Transition } \\
\text { (Curved to vertical) }\end{array}$ & $\begin{array}{c}\text { Vertical } \\
\text { (Top of patch on vertical) }\end{array}$ \\
\hline Right (East) $(\mathrm{cm})$ & 7.5 & 19.5 & 19.0 \\
\hline Left $($ West) $(\mathrm{cm})$ & 4.5 & 22.0 & 27.0 \\
\hline
\end{tabular}

Test Description: Q (film) - Rivulet Flow

Patch 2, $15 \mathrm{~cm}$ right of center, $16.5^{\circ}$

Pages 128 and 129 of SN-M\&O-SCI-043-V1

\begin{tabular}{|l|c|c|c|}
\hline $\begin{array}{l}\text { Position relative to drip } \\
\text { impact }\end{array}$ & $\mathbf{3 3}^{\mathbf{0}}$ & $\begin{array}{c}\text { Transition } \\
\text { (Curved to vertical) }\end{array}$ & $\begin{array}{c}\text { Vertical } \\
\text { (Top of patch on vertical) }\end{array}$ \\
\hline Right $($ East) $(\mathrm{cm})$ & 11.5 & 18.0 & 18.0 \\
\hline Left $($ West) $(\mathrm{cm})$ & 9.0 & 15.0 & 18.0 \\
\hline
\end{tabular}

Test Description: Q (film) - Rivulet Flow

Patch 5, $4 \mathrm{~cm}$ left of center, $16.5^{\circ}$

Pages 131 and 132 of SN-M\&O-SCI-043-V1

\begin{tabular}{|l|c|c|c|}
\hline $\begin{array}{l}\text { Position relative to drip } \\
\text { impact }\end{array}$ & $\mathbf{3 3}^{\mathbf{0}}$ & $\begin{array}{c}\text { Transition } \\
\text { (Curved to vertical) }\end{array}$ & $\begin{array}{c}\text { Vertical } \\
\text { (Top of patch on vertical) }\end{array}$ \\
\hline Right $($ East) $(\mathrm{cm})$ & 8.5 & N/A enter breach & N/A enter breach \\
\hline Left $($ West $)(\mathrm{cm})$ & 8.5 & N/A enter breach & N/A enter breach \\
\hline
\end{tabular}

Test Description: Q (film) - Rivulet Flow

Patch 2, $15 \mathrm{~cm}$ right of center, $33^{\circ}$

Pages 133 and 134 of SN-M\&O-SCI-043-V1

\begin{tabular}{|l|c|c|c|}
\hline $\begin{array}{l}\text { Position relative to drip } \\
\text { impact }\end{array}$ & $\mathbf{3 3}^{\mathbf{0}}$ & $\begin{array}{c}\text { Transition } \\
\text { (Curved to vertical) }\end{array}$ & $\begin{array}{c}\text { Vertical } \\
\text { (Top of patch on vertical) }\end{array}$ \\
\hline Right $($ East) $(\mathrm{cm})$ & N/A & 13.0 & 14.5 \\
\hline Left $($ West) $(\mathrm{cm})$ & N/A & 11.5 & 12.0 \\
\hline
\end{tabular}

Test Description: Q (film) - Rivulet Flow

Patch 2, center, $33^{\circ}$

Pages 135 and 136 of SN-M\&O-SCI-043-V1

\begin{tabular}{|l|c|c|c|}
\hline $\begin{array}{l}\text { Position relative to drip } \\
\text { impact }\end{array}$ & $\mathbf{3 3}^{\mathbf{0}}$ & $\begin{array}{c}\text { Transition } \\
\text { (Curved to vertical) }\end{array}$ & $\begin{array}{c}\text { Vertical } \\
\text { (Top of patch on vertical) }\end{array}$ \\
\hline Right $($ East) $(\mathrm{cm})$ & N/A & 9.0 & 6.0 \\
\hline Left $($ West) $(\mathrm{cm})$ & N/A & 10.5 & 18.0 \\
\hline
\end{tabular}


Test Description: Q (film) - Rivulet Flow

Patch 4, $8 \mathrm{~cm}$ right of center, $33^{\circ}$

Pages 137 and 138 of SN-M\&O-SCI-043-V1

\begin{tabular}{|l|c|c|c|}
\hline $\begin{array}{l}\text { Position relative to drip } \\
\text { impact }\end{array}$ & $\mathbf{3 3}^{\mathbf{0}}$ & $\begin{array}{c}\text { Transition } \\
\text { (Curved to vertical) }\end{array}$ & $\begin{array}{c}\text { Vertical } \\
\text { (Top of patch on vertical) }\end{array}$ \\
\hline Right $($ East) $(\mathrm{cm})$ & N/A & 14.5 top of patch 4 & N/A enter breach \\
\hline Left $($ West) $(\mathrm{cm})$ & N/A & 9.5 top of patch 4 & N/A enter breach \\
\hline
\end{tabular}

Test Description: Multiple Patch Tests, Smooth DS Surface

$81 \mathrm{~cm}$ left of DS center, $33^{\circ}$

Pages 142 and 143 of SN-M\&O-SCI-043-V1

\begin{tabular}{|l|c|c|c|}
\hline $\begin{array}{l}\text { Position relative to drip } \\
\text { impact }\end{array}$ & $\mathbf{3 3}^{\mathbf{0}}$ & $\begin{array}{c}\text { Transition } \\
\text { (Curved to vertical) }\end{array}$ & $\begin{array}{c}\text { Vertical } \\
\text { (Top of patch on vertical) }\end{array}$ \\
\hline Right $($ East) $(\mathrm{cm})$ & N/A & $14 \mathrm{~cm}$ & $15 \mathrm{~cm}$ \\
\hline Left $($ West) $(\mathrm{cm})$ & N/A & $12 \mathrm{~cm}$ & $18 \mathrm{~cm}$ \\
\hline
\end{tabular}

Test Description: Multiple Patch Tests, Smooth DS Surface

$27 \mathrm{~cm}$ left of DS center, $33^{\circ}$

Pages 144 and 145 of SN-M\&O-SCI-043-V1

\begin{tabular}{|l|c|c|c|}
\hline $\begin{array}{l}\text { Position relative to drip } \\
\text { impact }\end{array}$ & $\mathbf{3 3}^{\mathbf{0}}$ & $\begin{array}{c}\text { Transition } \\
\text { (Curved to vertical) }\end{array}$ & $\begin{array}{c}\text { Vertical } \\
\text { (Top of patch on vertical) }\end{array}$ \\
\hline Right $($ East) $(\mathrm{cm})$ & N/A & $11 \mathrm{~cm}$ & $16 \mathrm{~cm}$ \\
\hline Left $($ West) $(\mathrm{cm})$ & N/A & $9 \mathrm{~cm}$ & $14 \mathrm{~cm}$ \\
\hline
\end{tabular}

Test Description: Multiple Patch Tests, Smooth DS Surface

$27 \mathrm{~cm}$ right of DS center, $33^{\circ}$

Pages 146 and 147 of SN-M\&O-SCI-043-V1

\begin{tabular}{|l|c|c|c|}
\hline $\begin{array}{l}\text { Position relative to drip } \\
\text { impact }\end{array}$ & $\mathbf{3 3}^{\mathbf{0}}$ & $\begin{array}{c}\text { Transition } \\
\text { (Curved to vertical) }\end{array}$ & $\begin{array}{c}\text { Vertical } \\
\text { (Top of patch on vertical) }\end{array}$ \\
\hline Right $($ East) $(\mathrm{cm})$ & N/A & $10 \mathrm{~cm}$ & $8 \mathrm{~cm}$ \\
\hline Left $($ West $)(\mathrm{cm})$ & N/A & $13 \mathrm{~cm}$ & $15 \mathrm{~cm}$ \\
\hline
\end{tabular}

Test Description: Multiple Patch Tests, Smooth DS Surface

$81 \mathrm{~cm}$ right of DS center, $33^{\circ}$

Pages 148 and 149 of SN-M\&O-SCI-043-V1

\begin{tabular}{|l|c|c|c|c|}
\hline $\begin{array}{l}\text { Position relative to drip } \\
\text { impact }\end{array}$ & $\mathbf{3 3}^{\mathbf{0}}$ & $\begin{array}{c}\text { Transition } \\
\text { (Curved to vertical) }\end{array}$ & $\begin{array}{c}\text { Vertical } \\
\text { (Top of patch on vertical) }\end{array}$ \\
\hline Right $($ East) $(\mathrm{cm})$ & N/A & $10 \mathrm{~cm}$ & $22 \mathrm{~cm}$ \\
\hline Left $($ West $)(\mathrm{cm})$ & N/A & $10 \mathrm{~cm}$ & $10 \mathrm{~cm}$ \\
\hline
\end{tabular}


Test Description: Multiple Patch Tests, Smooth DS Surface

$27 \mathrm{~cm}$ right of drip shield center (Patch 6 center), crown

Pages 9 and 10 of Scientific Notebook SN-M\&O-SCI-043-V2

\begin{tabular}{|l|c|c|c|}
\hline $\begin{array}{l}\text { Position relative to drip } \\
\text { impact }\end{array}$ & $\mathbf{3 3}^{\mathbf{0}}$ & $\begin{array}{c}\text { Transition } \\
\text { (Curved to vertical) }\end{array}$ & $\begin{array}{c}\text { Vertical } \\
\text { (Top of patch on vertical) }\end{array}$ \\
\hline Right $($ East) $(\mathrm{cm})$ & 29.0 & Enter B5 & Enter B5 \\
\hline Left $($ West $)(\mathrm{cm})$ & 13.0 & 19.0 & 23.0 \\
\hline
\end{tabular}

Test Description: Multiple Patch Tests, Smooth DS Surface

$27 \mathrm{~cm}$ left of drip shield center, crown

Pages 15 and 16 of Scientific Notebook SN-M\&O-SCI-043-V2

\begin{tabular}{|l|c|c|c|}
\hline $\begin{array}{l}\text { Position relative to drip } \\
\text { impact }\end{array}$ & $\mathbf{3 3}^{\mathbf{0}}$ & $\begin{array}{c}\text { Transition } \\
\text { (Curved to vertical) }\end{array}$ & $\begin{array}{c}\text { Vertical } \\
\text { (Top of patch on vertical) }\end{array}$ \\
\hline Right $($ East) $(\mathrm{cm})$ & 21.5 & 29.0 & 30.0 \\
\hline Left $($ West $)(\mathrm{cm})$ & 21.0 & Enter B4 & Enter B4 \\
\hline
\end{tabular}

Test Description: Multiple Patch Tests, Smooth DS Surface

$81 \mathrm{~cm}$ left of drip shield center, crown

Pages 17 and 18 of Scientific Notebook SN-M\&O-SCI-043-V2

\begin{tabular}{|l|c|c|c|}
\hline $\begin{array}{l}\text { Position relative to drip } \\
\text { impact }\end{array}$ & $\mathbf{3 3}^{\mathbf{0}}$ & $\begin{array}{c}\text { Transition } \\
\text { (Curved to vertical) }\end{array}$ & $\begin{array}{c}\text { Vertical } \\
\text { (Top of patch on vertical) }\end{array}$ \\
\hline Right (East) $(\mathrm{cm})$ & 23.5 & Enter B4 & Enter B4 \\
\hline Left $($ West) $(\mathrm{cm})$ & 17.0 & 11.5 & 35.5 \\
\hline
\end{tabular}

Test Description: Multiple Patch Tests, Smooth DS Surface

$81 \mathrm{~cm}$ left of drip shield center, $16.5^{\circ}$

Pages 19 and 20 of Scientific Notebook SN-M\&O-SCI-043-V2

\begin{tabular}{|l|c|c|c|}
\hline $\begin{array}{l}\text { Position relative to drip } \\
\text { impact }\end{array}$ & $\mathbf{3 3}^{\mathbf{0}}$ & $\begin{array}{c}\text { Transition } \\
\text { (Curved to vertical) }\end{array}$ & $\begin{array}{c}\text { Vertical } \\
\text { (Top of patch on vertical) }\end{array}$ \\
\hline Right (East) $(\mathrm{cm})$ & 4.5 & Enter B4 & Enter B4 \\
\hline Left (West) $(\mathrm{cm})$ & 6.5 & 14.0 & Not Visible, Dry \\
\hline
\end{tabular}

Test Description: Multiple Patch Tests, Smooth DS Surface

$27 \mathrm{~cm}$ left of drip shield center, $16.5^{\circ}$

Pages 21 and 22 of Scientific Notebook SN-M\&O-SCI-043-V2

\begin{tabular}{|l|c|c|c|}
\hline $\begin{array}{l}\text { Position relative to drip } \\
\text { impact }\end{array}$ & $\mathbf{3 3}^{\mathbf{0}}$ & $\begin{array}{c}\text { Transition } \\
\text { (Curved to vertical) }\end{array}$ & $\begin{array}{c}\text { Vertical } \\
\text { (Top of patch on vertical) }\end{array}$ \\
\hline Right (East) $(\mathrm{cm})$ & 7.0 & 13.0 & 20.0 \\
\hline Left $($ West) $(\mathrm{cm})$ & 5.5 & 15.0 & 14.5 \\
\hline
\end{tabular}


Test Description: Multiple Patch Tests, Smooth DS Surface

$27 \mathrm{~cm}$ right of drip shield center, $16.5^{\circ}$

Pages 25 and 26 of Scientific Notebook SN-M\&O-SCI-043-V2

\begin{tabular}{|l|c|c|c|}
\hline $\begin{array}{l}\text { Position relative to drip } \\
\text { impact }\end{array}$ & $\mathbf{3 3}^{\mathbf{0}}$ & $\begin{array}{c}\text { Transition } \\
\text { (Curved to vertical) }\end{array}$ & $\begin{array}{c}\text { Vertical } \\
\text { (Top of patch on vertical) }\end{array}$ \\
\hline Right $($ East) $(\mathrm{cm})$ & 6.0 & 15.5 & 24.0 \\
\hline Left $($ West) $(\mathrm{cm})$ & 11.0 & 27.5 & 31.0 \\
\hline
\end{tabular}

Test Description: Multiple Patch Tests, Smooth DS Surface

$81 \mathrm{~cm}$ right of drip shield center, crown

Pages 200 and 201 of Scientific Notebook SN-M\&O-SCI-043-V2

\begin{tabular}{|l|c|c|c|}
\hline $\begin{array}{l}\text { Position relative to drip } \\
\text { impact }\end{array}$ & $\mathbf{3 3}^{\mathbf{0}}$ & $\begin{array}{c}\text { Transition } \\
\text { (Curved to vertical) }\end{array}$ & $\begin{array}{c}\text { Vertical } \\
\text { (Top of patch on vertical) }\end{array}$ \\
\hline Right (East) $(\mathrm{cm})$ & 18.0 & 17.0 & 29.0 \\
\hline Left $($ West) $(\mathrm{cm})$ & 20.0 & 21.0 & 32.0 \\
\hline
\end{tabular}

Test Description: Low Flow Rate $\left(0.2 \mathrm{~m}^{3} / \mathrm{yr}\right.$ nominal), Smooth DS Surface

$7 \mathrm{~cm}$ right of drip shield center, crown

Scientific Notebook SN-M\&O-SCI-043-V2, pp. 32-33

\begin{tabular}{|l|c|c|c|}
\hline $\begin{array}{l}\text { Position relative to drip } \\
\text { impact }\end{array}$ & $\mathbf{3 3}^{\mathbf{0}}$ & $\begin{array}{c}\text { Transition } \\
\text { (Curved to vertical) }\end{array}$ & $\begin{array}{c}\text { Vertical } \\
\text { (Top of patch on vertical) }\end{array}$ \\
\hline Right $($ East) $(\mathrm{cm})$ & 13.5 & 8.5 & 25.5 \\
\hline Left $($ West) $(\mathrm{cm})$ & 20.0 & 28.0 & 36.0 \\
\hline
\end{tabular}

Test Description: High Flow Rate $\left(20.0 \mathrm{~m}^{3} / \mathrm{yr}\right.$ nominal), Smooth DS Surface

$27 \mathrm{~cm}$ right of drip shield center, crown

Scientific Notebook SN-M\&O-SCI-043-V2, pp. 36-37

\begin{tabular}{|l|c|c|c|}
\hline $\begin{array}{l}\text { Position relative to drip } \\
\text { impact }\end{array}$ & $\mathbf{3 3}^{\mathbf{0}}$ & $\begin{array}{c}\text { Transition } \\
\text { (Curved to vertical) }\end{array}$ & $\begin{array}{c}\text { Vertical } \\
\text { (Top of patch on vertical) }\end{array}$ \\
\hline Right (East) $(\mathrm{cm})$ & 16.5 & 29.0 & 45.0 \\
\hline Left (West) $(\mathrm{cm})$ & 24.0 & 37.0 & 39.0 \\
\hline
\end{tabular}

Test Description: Low Flow Rate $\left(0.2 \mathrm{~m}^{3} / \mathrm{yr}\right.$ nominal), Smooth DS Surface

$27 \mathrm{~cm}$ left of drip shield center, crown

Scientific Notebook SN-M\&O-SCI-043-V2, pp. 38-39

\begin{tabular}{|l|c|c|c|}
\hline $\begin{array}{l}\text { Position relative to drip } \\
\text { impact }\end{array}$ & $\mathbf{3 3}^{\mathbf{0}}$ & $\begin{array}{c}\text { Transition } \\
\text { (Curved to vertical) }\end{array}$ & $\begin{array}{c}\text { Vertical } \\
\text { (Top of patch on vertical) }\end{array}$ \\
\hline Right (East) $(\mathrm{cm})$ & 8.0 & 13.0 & 20.0 \\
\hline Left $($ West) $(\mathrm{cm})$ & 10.0 & 23.0 & 24.0 \\
\hline
\end{tabular}


Test Description: High Flow Rate $\left(20.0 \mathrm{~m}^{3} / \mathrm{yr}\right.$ nominal), Smooth DS Surface

$27 \mathrm{~cm}$ left of drip shield center, crown

Scientific Notebook SN-M\&O-SCI-043-V2, pp. 40-41

\begin{tabular}{|l|c|c|c|}
\hline $\begin{array}{l}\text { Position relative to drip } \\
\text { impact }\end{array}$ & $\mathbf{3 3}^{\mathbf{0}}$ & $\begin{array}{c}\text { Transition } \\
\text { (Curved to vertical) }\end{array}$ & $\begin{array}{c}\text { Vertical } \\
\text { (Top of patch on vertical) }\end{array}$ \\
\hline Right (East) $(\mathrm{cm})$ & 19 & 27 & 34 \\
\hline Left (West) $(\mathrm{cm})$ & 24 & 32 & 21 \\
\hline
\end{tabular}

Test Description: Low Flow Rate $\left(0.2 \mathrm{~m}^{3} / \mathrm{yr}\right.$ nominal $)$, Smooth DS Surface

$54 \mathrm{~cm}$ left of drip shield center, crown

Scientific Notebook SN-M\&O-SCI-043-V2, pp. 42-43

\begin{tabular}{|l|c|c|c|}
\hline $\begin{array}{l}\text { Position relative to drip } \\
\text { impact }\end{array}$ & $\mathbf{3 3}^{\mathbf{0}}$ & $\begin{array}{c}\text { Transition } \\
\text { (Curved to vertical) }\end{array}$ & $\begin{array}{c}\text { Vertical } \\
\text { (Top of patch on vertical) }\end{array}$ \\
\hline Right (East) $(\mathrm{cm})$ & 30.0 & 54.0 & 63.0 \\
\hline Left (West) $(\mathrm{cm})$ & 10.5 & 15.0 & 15.0 \\
\hline
\end{tabular}

Test Description: High Flow Rate $\left(20.0 \mathrm{~m}^{3} / \mathrm{yr}\right.$ nominal), Smooth DS Surface

$54 \mathrm{~cm}$ left of drip shield center, crown

Scientific Notebook SN-M\&O-SCI-043-V2, pp. 44-45

\begin{tabular}{|l|c|c|c|}
\hline $\begin{array}{l}\text { Position relative to drip } \\
\text { impact }\end{array}$ & $\mathbf{3 3}^{\mathbf{0}}$ & $\begin{array}{c}\text { Transition } \\
\text { (Curved to vertical) }\end{array}$ & $\begin{array}{c}\text { Vertical } \\
\text { (Top of patch on vertical) }\end{array}$ \\
\hline Right $($ East) $(\mathrm{cm})$ & 32.0 & 44.0 & 56.0 \\
\hline Left (West) $(\mathrm{cm})$ & 22.0 & 30.0 & 35.0 \\
\hline
\end{tabular}

Test Description: High Flow Rate $\left(20.0 \mathrm{~m}^{3} / \mathrm{yr}\right.$ nominal), Smooth DS Surface

$54 \mathrm{~cm}$ left of drip shield center, $16.5^{\circ}$

Scientific Notebook SN-M\&O-SCI-043-V2, pp. 46-47

\begin{tabular}{|l|c|c|c|}
\hline $\begin{array}{l}\text { Position relative to drip } \\
\text { impact }\end{array}$ & $\mathbf{3 3}^{\mathbf{0}}$ & $\begin{array}{c}\text { Transition } \\
\text { (Curved to vertical) }\end{array}$ & $\begin{array}{c}\text { Vertical } \\
\text { (Top of patch on vertical) }\end{array}$ \\
\hline Right (East) $(\mathrm{cm})$ & 31.0 & 35.0 & 35.0 \\
\hline Left (West) $(\mathrm{cm})$ & 46.0 & 46.0 & 45.0 \\
\hline
\end{tabular}

Test Description: Low Flow Rate $\left(0.2 \mathrm{~m}^{3} / \mathrm{yr}\right.$ nominal), Smooth DS Surface

$54 \mathrm{~cm}$ left of drip shield center, $16.5^{\circ}$

Scientific Notebook SN-M\&O-SCI-043-V2, pp. 48-49

\begin{tabular}{|l|c|c|c|}
\hline $\begin{array}{l}\text { Position relative to drip } \\
\text { impact }\end{array}$ & $\mathbf{3 3}^{\mathbf{0}}$ & $\begin{array}{c}\text { Transition } \\
\text { (Curved to vertical) }\end{array}$ & $\begin{array}{c}\text { Vertical } \\
\text { (Top of patch on vertical) }\end{array}$ \\
\hline Right (East) $(\mathrm{cm})$ & 8.5 & 19.0 & 24.0 \\
\hline Left (West) $(\mathrm{cm})$ & 10.0 & 27.0 & 30.0 \\
\hline
\end{tabular}


Test Description: Low Flow Rate $\left(0.2 \mathrm{~m}^{3} / \mathrm{yr}\right.$ nominal), Smooth DS Surface

$27 \mathrm{~cm}$ left of drip shield center, $16.5^{\circ}$

Scientific Notebook SN-M\&O-SCI-043-V2, pp. 50-51

\begin{tabular}{|l|c|c|c|}
\hline $\begin{array}{l}\text { Position relative to drip } \\
\text { impact }\end{array}$ & $\mathbf{3 3}^{\mathbf{0}}$ & $\begin{array}{c}\text { Transition } \\
\text { (Curved to vertical) }\end{array}$ & $\begin{array}{c}\text { Vertical } \\
\text { (Top of patch on vertical) }\end{array}$ \\
\hline Right (East) $(\mathrm{cm})$ & 6.0 & 17.0 & 24.0 \\
\hline Left $($ West) $(\mathrm{cm})$ & 8.0 & 16.0 & 20.0 \\
\hline
\end{tabular}

Test Description: High Flow Rate $\left(20.0 \mathrm{~m}^{3} / \mathrm{yr}\right.$ nominal), Smooth DS Surface

$27 \mathrm{~cm}$ left of drip shield center, $16.5^{\circ}$

Scientific Notebook SN-M\&O-SCI-043-V2, pp. 52-53

\begin{tabular}{|l|c|c|c|}
\hline $\begin{array}{l}\text { Position relative to drip } \\
\text { impact }\end{array}$ & $\mathbf{3 3}^{\mathbf{0}}$ & $\begin{array}{c}\text { Transition } \\
\text { (Curved to vertical) }\end{array}$ & $\begin{array}{c}\text { Vertical } \\
\text { (Top of patch on vertical) }\end{array}$ \\
\hline Right (East) $(\mathrm{cm})$ & 18.0 & 22.0 & 25.0 \\
\hline Left (West) $(\mathrm{cm})$ & 24.0 & 19.0 & 21.0 \\
\hline
\end{tabular}

Test Description: High Flow Rate $\left(20.0 \mathrm{~m}^{3} / \mathrm{yr}\right.$ nominal), Smooth DS Surface

$27 \mathrm{~cm}$ right of drip shield center, $16.5^{\circ}$

Scientific Notebook SN-M\&O-SCI-043-V2, pp. 54-55

\begin{tabular}{|l|c|c|c|}
\hline $\begin{array}{l}\text { Position relative to drip } \\
\text { impact }\end{array}$ & $\mathbf{3 3}^{\mathbf{0}}$ & $\begin{array}{c}\text { Transition } \\
\text { (Curved to vertical) }\end{array}$ & $\begin{array}{c}\text { Vertical } \\
\text { (Top of patch on vertical) }\end{array}$ \\
\hline Right $($ East) $(\mathrm{cm})$ & 13.0 & 14.0 & 19.0 \\
\hline Left $($ West) $(\mathrm{cm})$ & 27.0 & 23.0 & 24.0 \\
\hline
\end{tabular}

Test Description: Low Flow Rate $\left(0.2 \mathrm{~m}^{3} / \mathrm{yr}\right.$ nominal $)$, Smooth DS Surface $27 \mathrm{~cm}$ right of drip shield center, $16.5^{\circ}$

Scientific Notebook SN-M\&O-SCI-043-V2, pp. 56-57

\begin{tabular}{|l|c|c|c|}
\hline $\begin{array}{l}\text { Position relative to drip } \\
\text { impact }\end{array}$ & $\mathbf{3 3}^{\mathbf{0}}$ & $\begin{array}{c}\text { Transition } \\
\text { (Curved to vertical) }\end{array}$ & $\begin{array}{c}\text { Vertical } \\
\text { (Top of patch on vertical) }\end{array}$ \\
\hline Right (East) $(\mathrm{cm})$ & 12.0 & 16.0 & 20.0 \\
\hline Left (West) $(\mathrm{cm})$ & 17.0 & 19.0 & 28.0 \\
\hline
\end{tabular}

Test Description: High Flow Rate $\left(20.0 \mathrm{~m}^{3} / \mathrm{yr}\right.$ nominal), Smooth DS Surface

$27 \mathrm{~cm}$ left of drip shield center, $33^{\circ}$

Scientific Notebook SN-M\&O-SCI-043-V2, pp. 58-59

\begin{tabular}{|l|c|c|c|}
\hline $\begin{array}{l}\text { Position relative to drip } \\
\text { impact }\end{array}$ & $\mathbf{3 3}^{\mathbf{0}}$ & $\begin{array}{c}\text { Transition } \\
\text { (Curved to vertical) }\end{array}$ & $\begin{array}{c}\text { Vertical } \\
\text { (Top of patch on vertical) }\end{array}$ \\
\hline Right (East) $(\mathrm{cm})$ & 4.0 & 11.0 & 26.0 \\
\hline Left (West) $(\mathrm{cm})$ & 3.0 & 17.0 & 18.0 \\
\hline
\end{tabular}


Test Description: High Flow Rate $\left(20.0 \mathrm{~m}^{3} / \mathrm{yr}\right.$ nominal), Smooth DS Surface

$27 \mathrm{~cm}$ right of drip shield center, $33^{\circ}$

Scientific Notebook SN-M\&O-SCI-043-V2, pp. 60-61

\begin{tabular}{|l|c|c|c|}
\hline $\begin{array}{l}\text { Position relative to drip } \\
\text { impact }\end{array}$ & $\mathbf{3 3}^{\mathbf{0}}$ & $\begin{array}{c}\text { Transition } \\
\text { (Curved to vertical) }\end{array}$ & $\begin{array}{c}\text { Vertical } \\
\text { (Top of patch on vertical) }\end{array}$ \\
\hline Right (East) $(\mathrm{cm})$ & 5.0 & 15.0 & 28.0 \\
\hline Left (West) $(\mathrm{cm})$ & 5.0 & 17.0 & 17.0 \\
\hline
\end{tabular}

Test Description: High Flow Rate $\left(20.0 \mathrm{~m}^{3} / \mathrm{yr}\right.$ nominal), Smooth DS Surface

$54 \mathrm{~cm}$ left of drip shield center, $33^{\circ}$

Scientific Notebook SN-M\&O-SCI-043-V2, pp. 62-63

\begin{tabular}{|l|c|c|c|}
\hline $\begin{array}{l}\text { Position relative to drip } \\
\text { impact }\end{array}$ & $\mathbf{3 3}^{\mathbf{0}}$ & $\begin{array}{c}\text { Transition } \\
\text { (Curved to vertical) }\end{array}$ & $\begin{array}{c}\text { Vertical } \\
\text { (Top of patch on vertical) }\end{array}$ \\
\hline Right (East) $(\mathrm{cm})$ & 4.0 & 17.0 & 18.0 \\
\hline Left (West) $(\mathrm{cm})$ & 4.0 & 17.0 & 20.0 \\
\hline
\end{tabular}

Test Description: Low Flow Rate $\left(0.2 \mathrm{~m}^{3} / \mathrm{yr}\right.$ nominal $)$, Smooth DS Surface

$54 \mathrm{~cm}$ left of drip shield center, $33^{\circ}$

Scientific Notebook SN-M\&O-SCI-043-V2, pp. 64-65

\begin{tabular}{|l|c|c|c|}
\hline $\begin{array}{l}\text { Position relative to drip } \\
\text { impact }\end{array}$ & $\mathbf{3 3}^{\mathbf{0}}$ & $\begin{array}{c}\text { Transition } \\
\text { (Curved to vertical) }\end{array}$ & $\begin{array}{c}\text { Vertical } \\
\text { (Top of patch on vertical) }\end{array}$ \\
\hline Right (East) $(\mathrm{cm})$ & NA & NA & NA \\
\hline Left (West) $(\mathrm{cm})$ & NA & NA & NA \\
\hline
\end{tabular}

Test Description: Low Flow Rate $\left(0.2 \mathrm{~m}^{3} / \mathrm{yr}\right.$ nominal), Smooth DS Surface

$27 \mathrm{~cm}$ left of drip shield center, $33^{\circ}$

Scientific Notebook SN-M\&O-SCI-043-V2, pp. 66-67

\begin{tabular}{|l|c|c|c|}
\hline $\begin{array}{l}\text { Position relative to drip } \\
\text { impact }\end{array}$ & $\mathbf{3 3}^{\mathbf{0}}$ & $\begin{array}{c}\text { Transition } \\
\text { (Curved to vertical) }\end{array}$ & $\begin{array}{c}\text { Vertical } \\
\text { (Top of patch on vertical) }\end{array}$ \\
\hline Right (East) $(\mathrm{cm})$ & NA & 9.0 & Enter B4 \\
\hline Left (West) $(\mathrm{cm})$ & NA & 9.5 & 8.0 \\
\hline
\end{tabular}

Test Description: Low Flow Rate $\left(0.2 \mathrm{~m}^{3} / \mathrm{yr}\right.$ nominal $)$, Smooth DS Surface

$27 \mathrm{~cm}$ right of drip shield center, $33^{\circ}$

Scientific Notebook SN-M\&O-SCI-043-V2, pp. 68-69

\begin{tabular}{|l|c|c|c|}
\hline $\begin{array}{l}\text { Position relative to drip } \\
\text { impact }\end{array}$ & $\mathbf{3 3}^{\mathbf{0}}$ & $\begin{array}{c}\text { Transition } \\
\text { (Curved to vertical) }\end{array}$ & $\begin{array}{c}\text { Vertical } \\
\text { (Top of patch on vertical) }\end{array}$ \\
\hline Right $($ East) $(\mathrm{cm})$ & NA & 8.5 & 12.0 \\
\hline Left $($ West) $(\mathrm{cm})$ & NA & 10.0 & 8.0 \\
\hline
\end{tabular}


Test Description: Single Patch q(splash) Tests, Rough DS Surface

Patch 6, $20 \mathrm{~cm}$ left of patch center, crown

Scientific Notebook SN-M\&O-SCI-043-V2, pp. 93-94

\begin{tabular}{|l|c|c|c|}
\hline $\begin{array}{l}\text { Position relative to drip } \\
\text { impact }\end{array}$ & $\mathbf{3 3}^{\mathbf{0}}$ & $\begin{array}{c}\text { Transition } \\
\text { (Curved to vertical) }\end{array}$ & $\begin{array}{c}\text { Vertical } \\
\text { (Top of patch on vertical) }\end{array}$ \\
\hline Right (East) $(\mathrm{cm})$ & 16.0 & 9.5 & 19.0 \\
\hline Left $($ West) $(\mathrm{cm})$ & 14.0 & 12.5 & 12.5 \\
\hline
\end{tabular}

Test Description: Single Patch q(splash) Tests, Rough DS Surface

Patch 6 center, $15 \mathrm{~cm}$ below crown

Scientific Notebook SN-M\&O-SCI-043-V2, pp. 95-96

\begin{tabular}{|l|c|c|c|}
\hline $\begin{array}{l}\text { Position relative to drip } \\
\text { impact }\end{array}$ & $\mathbf{3 3}^{\mathbf{0}}$ & $\begin{array}{c}\text { Transition } \\
\text { (Curved to vertical) }\end{array}$ & $\begin{array}{c}\text { Vertical } \\
\text { (Top of patch on vertical) }\end{array}$ \\
\hline Right (East) $(\mathrm{cm})$ & 6.0 & 9.0 & 11.0 \\
\hline Left $($ West) $(\mathrm{cm})$ & 10.0 & 9.5 & 10.5 \\
\hline
\end{tabular}

Test Description: Single Patch q(splash) Tests, Rough DS Surface

Patch 6, patch center, $16.5^{\circ}$

Scientific Notebook SN-M\&O-SCI-043-V2, pp. 97-98

\begin{tabular}{|l|c|c|c|}
\hline $\begin{array}{l}\text { Position relative to drip } \\
\text { impact }\end{array}$ & $\mathbf{3 3}^{\mathbf{0}}$ & $\begin{array}{c}\text { Transition } \\
\text { (Curved to vertical) }\end{array}$ & $\begin{array}{c}\text { Vertical } \\
\text { (Top of patch on vertical) }\end{array}$ \\
\hline Right (East) $(\mathrm{cm})$ & 13.0 & 43.0 & 39.0 \\
\hline Left (West) $(\mathrm{cm})$ & 8.0 & 39.0 & 38.0 \\
\hline
\end{tabular}

Test Description: Single Patch q(splash) Tests, Rough DS Surface

Patch 5, $20 \mathrm{~cm}$ left of patch center, $16.5^{\circ}$

Scientific Notebook SN-M\&O-SCI-043-V2, pp. 99-100

\begin{tabular}{|l|c|c|c|}
\hline $\begin{array}{l}\text { Position relative to drip } \\
\text { impact }\end{array}$ & $\mathbf{3 3}^{\mathbf{0}}$ & $\begin{array}{c}\text { Transition } \\
\text { (Curved to vertical) }\end{array}$ & $\begin{array}{c}\text { Vertical } \\
\text { (Top of patch on vertical) }\end{array}$ \\
\hline Right (East) $(\mathrm{cm})$ & 29.0 & 2.0 & 4.0 \\
\hline Left (West) $(\mathrm{cm})$ & 27.0 & 40.0 & 34.0 \\
\hline
\end{tabular}

Test Description: Single Patch q(splash) Tests, Rough DS Surface

Patch 5, $20 \mathrm{~cm}$ left of patch center, $33^{\circ}$

Scientific Notebook SN-M\&O-SCI-043-V2, pp. 101-102

\begin{tabular}{|l|c|c|c|}
\hline $\begin{array}{l}\text { Position relative to drip } \\
\text { impact }\end{array}$ & $\mathbf{3 3}^{\mathbf{0}}$ & $\begin{array}{c}\text { Transition } \\
\text { (Curved to vertical) }\end{array}$ & $\begin{array}{c}\text { Vertical } \\
\text { (Top of patch on vertical) }\end{array}$ \\
\hline Right $($ East) $(\mathrm{cm})$ & 3.0 & 3.0 & 3.0 \\
\hline Left $($ West) $(\mathrm{cm})$ & 1.0 & 16.0 & 18.0 \\
\hline
\end{tabular}


Test Description: Single Patch q(splash) Tests, Rough DS Surface

Patch 4, $27 \mathrm{~cm}$ right of patch center, $33^{\circ}$

Scientific Notebook SN-M\&O-SCI-043-V2, pp. 103-104

\begin{tabular}{|l|c|c|c|}
\hline $\begin{array}{l}\text { Position relative to drip } \\
\text { impact }\end{array}$ & $\mathbf{3 3}^{\mathbf{0}}$ & $\begin{array}{c}\text { Transition } \\
\text { (Curved to vertical) }\end{array}$ & $\begin{array}{c}\text { Vertical } \\
\text { (Top of patch on vertical) }\end{array}$ \\
\hline Right (East) $(\mathrm{cm})$ & 3.0 & 17.0 & 16.0 \\
\hline Left $($ West) $(\mathrm{cm})$ & 3.0 & 11.5 & 10.0 \\
\hline
\end{tabular}

Test Description: Single Patch q(film) Rivulet Flow Tests: Rough DS Surface

Patch 4, centerline, $33^{\circ}$

Pages 110 and 111 of Scientific Notebook SN-M\&O-SCI-043-V2

\begin{tabular}{|l|c|c|c|}
\hline $\begin{array}{l}\text { Position relative to drip } \\
\text { impact }\end{array}$ & $\mathbf{3 3}^{\mathbf{0}}$ & $\begin{array}{c}\text { Transition } \\
\text { (Curved to vertical) }\end{array}$ & $\begin{array}{c}\text { Vertical } \\
\text { (Top of patch on vertical) }\end{array}$ \\
\hline Right (East) $(\mathrm{cm})$ & 3.0 & 23.0 & 18.0 \\
\hline Left (West) $(\mathrm{cm})$ & 2.0 & 20.0 & 22.0 \\
\hline
\end{tabular}

Test Description: Single Patch q(film) Rivulet Flow Tests: Rough DS Surface

Patch 2, center, $33^{\circ}$

Pages 112 and 113 of Scientific Notebook SN-M\&O-SCI-043-V2

\begin{tabular}{|l|c|c|c|}
\hline $\begin{array}{l}\text { Position relative to drip } \\
\text { impact }\end{array}$ & $\mathbf{3 3}^{\mathbf{0}}$ & $\begin{array}{c}\text { Transition } \\
\text { (Curved to vertical) }\end{array}$ & $\begin{array}{c}\text { Vertical } \\
\text { (Top of patch on vertical) }\end{array}$ \\
\hline Right (East) $(\mathrm{cm})$ & 2.0 & 2.0 & 4.0 \\
\hline Left (West) $(\mathrm{cm})$ & 2.0 & 9.0 & 8.0 \\
\hline
\end{tabular}

Test Description: Single Patch q(film) Rivulet Flow Tests: Rough DS Surface

Patch 2, $13.5 \mathrm{~cm}$ right of center, $33^{\circ}$

Pages 114 and 115 of Scientific Notebook SN-M\&O-SCI-043-V2

\begin{tabular}{|l|c|c|c|}
\hline $\begin{array}{l}\text { Position relative to drip } \\
\text { impact }\end{array}$ & $\mathbf{3 3}^{\mathbf{0}}$ & $\begin{array}{c}\text { Transition } \\
\text { (Curved to vertical) }\end{array}$ & $\begin{array}{c}\text { Vertical } \\
\text { (Top of patch on vertical) }\end{array}$ \\
\hline Right (East) $(\mathrm{cm})$ & 2.0 & 10.0 & 10.0 \\
\hline Left (West) $(\mathrm{cm})$ & 2.0 & 1.0 & 3.0 \\
\hline
\end{tabular}

Test Description: Single Patch q(film) Rivulet Flow Tests: Rough DS Surface

Patch 5, centerline, $16.5^{\circ}$

Pages 116 and 117 of Scientific Notebook SN-M\&O-SCI-043-V2

\begin{tabular}{|l|c|c|c|}
\hline $\begin{array}{l}\text { Position relative to drip } \\
\text { impact }\end{array}$ & $\mathbf{3 3}^{\mathbf{0}}$ & $\begin{array}{c}\text { Transition } \\
\text { (Curved to vertical) }\end{array}$ & $\begin{array}{c}\text { Vertical } \\
\text { (Top of patch on vertical) }\end{array}$ \\
\hline Right (East) $(\mathrm{cm})$ & 16.5 & 18.0 & 19.0 \\
\hline Left (West) $(\mathrm{cm})$ & 27.0 & 22.0 & 22.0 \\
\hline
\end{tabular}


Test Description: Single Patch q(film) Rivulet Flow Tests: Rough DS Surface

Patch 2, $13.5 \mathrm{~cm}$ right of centerline, $16.5^{\circ}$

Pages 118 and 119 of Scientific Notebook SN-M\&O-SCI-043-V2

\begin{tabular}{|l|c|c|c|}
\hline $\begin{array}{l}\text { Position relative to drip } \\
\text { impact }\end{array}$ & $\mathbf{3 3}^{\mathbf{0}}$ & $\begin{array}{c}\text { Transition } \\
\text { (Curved to vertical) }\end{array}$ & $\begin{array}{c}\text { Vertical } \\
\text { (Top of patch on vertical) }\end{array}$ \\
\hline Right (East) $(\mathrm{cm})$ & 14.0 & 25.0 & 20.0 \\
\hline Left (West) $(\mathrm{cm})$ & 12.0 & 14.0 & 12.0 \\
\hline
\end{tabular}

Test Description: Single Patch q(film) Rivulet Flow Tests: Rough DS Surface

Patch 5, $13.5 \mathrm{~cm}$ left of patch center, $16.5^{\circ}$

Pages 120 and 121 of Scientific Notebook SN-M\&O-SCI-043-V2

\begin{tabular}{|l|c|c|c|}
\hline $\begin{array}{l}\text { Position relative to drip } \\
\text { impact }\end{array}$ & $\mathbf{3 3}^{\mathbf{0}}$ & $\begin{array}{c}\text { Transition } \\
\text { (Curved to vertical) }\end{array}$ & $\begin{array}{c}\text { Vertical } \\
\text { (Top of patch on vertical) }\end{array}$ \\
\hline Right (East) $(\mathrm{cm})$ & 20.5 & $\mathrm{~N} / \mathrm{A}$ & N/A \\
\hline Left (West) $(\mathrm{cm})$ & 15.0 & 15.0 & 15.5 \\
\hline
\end{tabular}

Test Description: Single Patch q(film) Rivulet Flow Tests: Rough DS Surface

Patch 2, centerline, $16.5^{\circ}$

Pages 122 and 123 of Scientific Notebook SN-M\&O-SCI-043-V2

\begin{tabular}{|l|c|c|c|}
\hline $\begin{array}{l}\text { Position relative to drip } \\
\text { impact }\end{array}$ & $\mathbf{3 3}^{\mathbf{0}}$ & $\begin{array}{c}\text { Transition } \\
\text { (Curved to vertical) }\end{array}$ & $\begin{array}{c}\text { Vertical } \\
\text { (Top of patch on vertical) }\end{array}$ \\
\hline Right (East) $(\mathrm{cm})$ & 32.0 & 32.0 & 41.0 \\
\hline Left (West) $(\mathrm{cm})$ & 20.0 & 20.0 & 18.0 \\
\hline
\end{tabular}

Test Description: Single Patch q(film) Rivulet Flow Tests: Rough DS Surface

Patch 4, centerline, $16.5^{\circ}$

Pages 126 and 127 of Scientific Notebook SN-M\&O-SCI-043-V2

\begin{tabular}{|l|c|c|c|}
\hline $\begin{array}{l}\text { Position relative to drip } \\
\text { impact }\end{array}$ & $\mathbf{3 3}^{\mathbf{0}}$ & $\begin{array}{c}\text { Transition } \\
\text { (Curved to vertical) }\end{array}$ & $\begin{array}{c}\text { Vertical } \\
\text { (Top of patch on vertical) }\end{array}$ \\
\hline Right (East) $(\mathrm{cm})$ & 11.0 & $\mathrm{~N} / \mathrm{A}$ & N/A \\
\hline Left (West) $(\mathrm{cm})$ & 23.5 & 25.0 & 26.5 \\
\hline
\end{tabular}

Test Description: Single Patch q(film) Rivulet Flow Tests: Rough DS Surface

Patch 4, centerline, crown

Pages 128 and 129 of Scientific Notebook SN-M\&O-SCI-043-V2

\begin{tabular}{|l|c|c|c|}
\hline $\begin{array}{l}\text { Position relative to drip } \\
\text { impact }\end{array}$ & $\mathbf{3 3}^{\mathbf{0}}$ & $\begin{array}{c}\text { Transition } \\
\text { (Curved to vertical) }\end{array}$ & $\begin{array}{c}\text { Vertical } \\
\text { (Top of patch on vertical) }\end{array}$ \\
\hline Right (East) $(\mathrm{cm})$ & 1.0 & N/A & N/A \\
\hline Left (West) $(\mathrm{cm})$ & 26.0 & 27.0 & 26.5 \\
\hline
\end{tabular}


Test Description: Single Patch q(film) Rivulet Flow Tests: Rough DS Surface

Patch 4, $13.5 \mathrm{~cm}$ right of patch center, crown

Pages 132 and 133 of Scientific Notebook SN-M\&O-SCI-043-V2

\begin{tabular}{|l|c|c|c|}
\hline $\begin{array}{l}\text { Position relative to drip } \\
\text { impact }\end{array}$ & $\mathbf{3 3}^{\mathbf{0}}$ & $\begin{array}{c}\text { Transition } \\
\text { (Curved to vertical) }\end{array}$ & $\begin{array}{c}\text { Vertical } \\
\text { (Top of patch on vertical) }\end{array}$ \\
\hline Right (East) $(\mathrm{cm})$ & 21.0 & 22.0 & 23.0 \\
\hline Left $($ West $)(\mathrm{cm})$ & 11.5 & N/A & N/A \\
\hline
\end{tabular}

Test Description: Single Patch q(film) Rivulet Flow Tests: Rough DS Surface

Patch 2, centerline $10.5 \mathrm{~cm}$ forward from crown, Patch 6 covered

Pages 134 and 135 of Scientific Notebook SN-M\&O-SCI-043-V2

\begin{tabular}{|l|c|c|c|}
\hline $\begin{array}{l}\text { Position relative to drip } \\
\text { impact }\end{array}$ & $\mathbf{3 3}^{\mathbf{0}}$ & $\begin{array}{c}\text { Transition } \\
\text { (Curved to vertical) }\end{array}$ & $\begin{array}{c}\text { Vertical } \\
\text { (Top of patch on vertical) }\end{array}$ \\
\hline Right (East) $(\mathrm{cm})$ & $20.0 \mathrm{ends}$ & 12.0 & 13.5 \\
\hline Left (West) $(\mathrm{cm})$ & 11.5 & 11.5 & 12.0 \\
\hline
\end{tabular}

Test Description: Single Patch q(film) Rivulet Flow Tests: Rough DS Surface

Patch 2, $13.5 \mathrm{~cm}$ left of center, $10.5 \mathrm{~cm}$ forward from crown

Pages 136 and 137 of Scientific Notebook SN-M\&O-SCI-043-V2

\begin{tabular}{|l|c|c|c|}
\hline $\begin{array}{l}\text { Position relative to drip } \\
\text { impact }\end{array}$ & $\mathbf{3 3}^{\mathbf{0}}$ & $\begin{array}{c}\text { Transition } \\
\text { (Curved to vertical) }\end{array}$ & $\begin{array}{c}\text { Vertical } \\
\text { (Top of patch on vertical) }\end{array}$ \\
\hline Right (East) $(\mathrm{cm})$ & 25.0 & 22.0 & 23.0 \\
\hline Left $($ West) $(\mathrm{cm})$ & 20.0 & 19.0 & $\begin{array}{c}\text { Not visible beyond } \\
\text { transition }\end{array}$ \\
\hline
\end{tabular}

Test Description: Single Patch q(film) Rivulet Flow Tests: Rough DS Surface

Patch 5, $13.5 \mathrm{~cm}$ left of patch center, crown

Pages 138 and 139 of Scientific Notebook SN-M\&O-SCI-043-V2

\begin{tabular}{|l|c|c|c|}
\hline $\begin{array}{l}\text { Position relative to drip } \\
\text { impact }\end{array}$ & $\mathbf{3 3}^{\mathbf{0}}$ & $\begin{array}{c}\text { Transition } \\
\text { (Curved to vertical) }\end{array}$ & $\begin{array}{c}\text { Vertical } \\
\text { (Top of patch on vertical) }\end{array}$ \\
\hline Right (East) $(\mathrm{cm})$ & 22.0 & N/A & N/A \\
\hline Left (West) $(\mathrm{cm})$ & 8.0 & 11.5 & 11.5 \\
\hline
\end{tabular}

Test Description: Single Patch q(film) Rivulet Flow Tests: Rough DS Surface

Patch 5, $13.5 \mathrm{~cm}$ left of patch center, $33^{\circ}$

Pages 142 and 143 of Scientific Notebook SN-M\&O-SCI-043-V2

\begin{tabular}{|l|c|c|c|}
\hline $\begin{array}{l}\text { Position relative to drip } \\
\text { impact }\end{array}$ & $\mathbf{3 3}^{\mathbf{0}}$ & $\begin{array}{c}\text { Transition } \\
\text { (Curved to vertical) }\end{array}$ & $\begin{array}{c}\text { Vertical } \\
\text { (Top of patch on vertical) }\end{array}$ \\
\hline Right (East) $(\mathrm{cm})$ & N/A & N/A & N/A \\
\hline Left $($ West $)(\mathrm{cm})$ & N/A & 9.0 & 9.0 \\
\hline
\end{tabular}


Test Description: Single Patch q(film) Rivulet Flow Tests: Rough DS Surface

Patch 4, $13.5 \mathrm{~cm}$ right of patch center, $33^{\circ}$

Pages 144 and 145 of Scientific Notebook SN-M\&O-SCI-043-V2

\begin{tabular}{|l|c|c|c|}
\hline $\begin{array}{l}\text { Position relative to drip } \\
\text { impact }\end{array}$ & $\mathbf{3 3}^{\mathbf{0}}$ & $\begin{array}{c}\text { Transition } \\
\text { (Curved to vertical) }\end{array}$ & $\begin{array}{c}\text { Vertical } \\
\text { (Top of patch on vertical) }\end{array}$ \\
\hline Right (East) $(\mathrm{cm})$ & N/A & 9.0 & 11.0 \\
\hline Left $($ West) $(\mathrm{cm})$ & N/A & N/A & N/A \\
\hline
\end{tabular}

Test Description: Multiple Patch Tests, Rough DS Surface

$81 \mathrm{~cm}$ left of drip shield center, Crown

Pages 149 and 150 of Scientific Notebook SN-M\&O-SCI-043-V2

\begin{tabular}{|l|c|c|c|}
\hline $\begin{array}{l}\text { Position relative to drip } \\
\text { impact }\end{array}$ & $\mathbf{3 3}^{\mathbf{0}}$ & $\begin{array}{c}\text { Transition } \\
\text { (Curved to vertical) }\end{array}$ & $\begin{array}{c}\text { Vertical } \\
\text { (Top of patch on vertical) }\end{array}$ \\
\hline Right (East) $(\mathrm{cm})$ & 17.5 & Enter Breach & Enter Breach \\
\hline Left (West) $(\mathrm{cm})$ & 32.5 & 30.0 & 32.0 \\
\hline
\end{tabular}

Test Description: Multiple Patch Tests, Rough DS Surface

$27 \mathrm{~cm}$ left of drip shield center, Crown

Pages 151 and 152 of Scientific Notebook SN-M\&O-SCI-043-V2

\begin{tabular}{|l|c|c|c|}
\hline $\begin{array}{l}\text { Position relative to drip } \\
\text { impact }\end{array}$ & $\mathbf{3 3}^{\mathbf{0}}$ & $\begin{array}{c}\text { Transition } \\
\text { (Curved to vertical) }\end{array}$ & $\begin{array}{c}\text { Vertical } \\
\text { (Top of patch on vertical) }\end{array}$ \\
\hline Right $($ East) $(\mathrm{cm})$ & 18.0 & 18.0 & 18.5 \\
\hline Left $($ West) $(\mathrm{cm})$ & 21.5 & Enter B4 & Enter B4 \\
\hline
\end{tabular}

Test Description: Multiple Patch Tests, Rough DS Surface

$27 \mathrm{~cm}$ right of drip shield center, Crown

Pages 153 and 154 of Scientific Notebook SN-M\&O-SCI-043-V2

\begin{tabular}{|l|c|c|c|}
\hline $\begin{array}{l}\text { Position relative to drip } \\
\text { impact }\end{array}$ & $\mathbf{3 3}^{\mathbf{0}}$ & $\begin{array}{c}\text { Transition } \\
\text { (Curved to vertical) }\end{array}$ & $\begin{array}{c}\text { Vertical } \\
\text { (Top of patch on vertical) }\end{array}$ \\
\hline Right (East) $(\mathrm{cm})$ & 10.0 & 7.5 & 9.5 \\
\hline Left (West) $(\mathrm{cm})$ & 10.0 & 10.0 & Rivulet ends, $4.0 \mathrm{next}$ \\
\hline
\end{tabular}

Test Description: Multiple Patch Tests, Rough DS Surface

$27 \mathrm{~cm}$ right of drip shield center, Crown

Pages 155 and 156 of Scientific Notebook SN-M\&O-SCI-043-V2

\begin{tabular}{|l|c|c|c|}
\hline $\begin{array}{l}\text { Position relative to drip } \\
\text { impact }\end{array}$ & $\mathbf{3 3}^{\mathbf{0}}$ & $\begin{array}{c}\text { Transition } \\
\text { (Curved to vertical) }\end{array}$ & $\begin{array}{c}\text { Vertical } \\
\text { (Top of patch on vertical) }\end{array}$ \\
\hline Right (East) $(\mathrm{cm})$ & None & None & None \\
\hline Left (West) $(\mathrm{cm})$ & 1.0 & 1.0 & 0.5 \\
\hline
\end{tabular}


Test Description: Multiple Patch Tests, Rough DS Surface

$81 \mathrm{~cm}$ right of drip shield center, Crown

Pages 157 and 158 of Scientific Notebook SN-M\&O-SCI-043-V2

\begin{tabular}{|l|c|c|c|}
\hline $\begin{array}{l}\text { Position relative to drip } \\
\text { impact }\end{array}$ & $\mathbf{3 3}^{\mathbf{0}}$ & $\begin{array}{c}\text { Transition } \\
\text { (Curved to vertical) }\end{array}$ & $\begin{array}{c}\text { Vertical } \\
\text { (Top of patch on vertical) }\end{array}$ \\
\hline Right $($ East) $(\mathrm{cm})$ & $* 34.0$ & $* 32.0$ & 25.0 \\
\hline Left $($ West $)(\mathrm{cm})$ & 17.0 & Enter B5 & Enter B5 \\
\hline
\end{tabular}

Test Description: Multiple Patch Tests, Rough DS Surface

$27 \mathrm{~cm}$ right of drip shield center, $16.5^{\circ}$

Pages 161 and 162 of Scientific Notebook SN-M\&O-SCI-043-V2

\begin{tabular}{|l|c|c|c|}
\hline $\begin{array}{l}\text { Position relative to drip } \\
\text { impact }\end{array}$ & $\mathbf{3 3}^{\mathbf{0}}$ & $\begin{array}{c}\text { Transition } \\
\text { (Curved to vertical) }\end{array}$ & $\begin{array}{c}\text { Vertical } \\
\text { (Top of patch on vertical) }\end{array}$ \\
\hline Right (East) $(\mathrm{cm})$ & 12.0 & 8.0 & 7.0 \\
\hline Left (West) $(\mathrm{cm})$ & 8.0 & 6.0 & 6.0 \\
\hline
\end{tabular}

Test Description: Multiple Patch Tests, Rough DS Surface

$27 \mathrm{~cm}$ left of drip shield center, $16.5^{\circ}$

Pages 163 and 164 of Scientific Notebook SN-M\&O-SCI-043-V2

\begin{tabular}{|l|c|c|c|}
\hline $\begin{array}{l}\text { Position relative to drip } \\
\text { impact }\end{array}$ & $\mathbf{3 3}^{\mathbf{0}}$ & $\begin{array}{c}\text { Transition } \\
\text { (Curved to vertical) }\end{array}$ & $\begin{array}{c}\text { Vertical } \\
\text { (Top of patch on vertical) }\end{array}$ \\
\hline Right $($ East) $(\mathrm{cm})$ & 19.0 & 13.0 & 13.0 \\
\hline Left $($ West) $(\mathrm{cm})$ & 21.0 & 12.0 & 10.0 \\
\hline
\end{tabular}

Test Description: Multiple Patch Tests, Rough DS Surface

$81 \mathrm{~cm}$ left of drip shield center, $16.5^{\circ}$

Pages 165 and 166 of Scientific Notebook SN-M\&O-SCI-043-V2

\begin{tabular}{|l|c|c|c|}
\hline $\begin{array}{l}\text { Position relative to drip } \\
\text { impact }\end{array}$ & $\mathbf{3 3}^{\mathbf{0}}$ & $\begin{array}{c}\text { Transition } \\
\text { (Curved to vertical) }\end{array}$ & $\begin{array}{c}\text { Vertical } \\
\text { (Top of patch on vertical) }\end{array}$ \\
\hline Right (East) $(\mathrm{cm})$ & 22.0 & 12.0 & 13.0 \\
\hline Left (West) $(\mathrm{cm})$ & 16.0 & 14.0 & 9.0 \\
\hline
\end{tabular}

Test Description: Multiple Patch Tests, Rough DS Surface

$81 \mathrm{~cm}$ right of drip shield center, $33^{\circ}$

Pages 167 and 168 of Scientific Notebook SN-M\&O-SCI-043-V2

\begin{tabular}{|l|c|c|c|}
\hline $\begin{array}{l}\text { Position relative to drip } \\
\text { impact }\end{array}$ & $\mathbf{3 3}^{\mathbf{0}}$ & $\begin{array}{c}\text { Transition } \\
\text { (Curved to vertical) }\end{array}$ & $\begin{array}{c}\text { Vertical } \\
\text { (Top of patch on vertical) }\end{array}$ \\
\hline Right (East) $(\mathrm{cm})$ & N/A & 2.0 & 1.5 \\
\hline Left (West) $(\mathrm{cm})$ & N/A & 2.0 & 2.0 \\
\hline
\end{tabular}


Test Description: Multiple Patch Tests, Rough DS Surface

$27 \mathrm{~cm}$ right of drip shield center, $33^{\circ}$

Pages 169 and 170 of Scientific Notebook SN-M\&O-SCI-043-V2

\begin{tabular}{|l|c|c|c|}
\hline $\begin{array}{l}\text { Position relative to drip } \\
\text { impact }\end{array}$ & $\mathbf{3 3}^{\mathbf{0}}$ & $\begin{array}{c}\text { Transition } \\
\text { (Curved to vertical) }\end{array}$ & $\begin{array}{c}\text { Vertical } \\
\text { (Top of patch on vertical) }\end{array}$ \\
\hline Right $($ East) $(\mathrm{cm})$ & N/A & 1.0 & 1.0 \\
\hline Left $($ West $)(\mathrm{cm})$ & N/A & 3.0 & 2.5 \\
\hline
\end{tabular}

Test Description: Multiple Patch Tests, Rough DS Surface

$27 \mathrm{~cm}$ left of drip shield center, $33^{\circ}$

Pages 171 and 172 of Scientific Notebook SN-M\&O-SCI-043-V2

\begin{tabular}{|l|c|c|c|}
\hline $\begin{array}{l}\text { Position relative to drip } \\
\text { impact }\end{array}$ & $\mathbf{3 3}^{\mathbf{0}}$ & $\begin{array}{c}\text { Transition } \\
\text { (Curved to vertical) }\end{array}$ & $\begin{array}{c}\text { Vertical } \\
\text { (Top of patch on vertical) }\end{array}$ \\
\hline Right (East) $(\mathrm{cm})$ & N/A & 1.0 & 1.5 \\
\hline Left (West) $(\mathrm{cm})$ & N/A & 2.0 & 4.0 \\
\hline
\end{tabular}

Test Description: Multiple Patch Tests, Rough DS Surface

$81 \mathrm{~cm}$ left of drip shield center, $33^{\circ}$

Pages 173 and 174 of Scientific Notebook SN-M\&O-SCI-043-V2

\begin{tabular}{|l|c|c|c|}
\hline $\begin{array}{l}\text { Position relative to drip } \\
\text { impact }\end{array}$ & $\mathbf{3 3}^{\mathbf{0}}$ & $\begin{array}{c}\text { Transition } \\
\text { (Curved to vertical) }\end{array}$ & $\begin{array}{c}\text { Vertical } \\
\text { (Top of patch on vertical) }\end{array}$ \\
\hline Right (East) $(\mathrm{cm})$ & N/A & 4.0 & 2.0 \\
\hline Left (West) $(\mathrm{cm})$ & N/A & 3.0 & 1.0 \\
\hline
\end{tabular}

Test Description: High Flow Rate $\left(0.2 \mathrm{~m}^{3} / \mathrm{yr}\right.$ nominal $)$, Rough DS Surface

$27 \mathrm{~cm}$ left of drip shield center, $33^{0}$

Scientific Notebook SN-M\&O-SCI-043-V2, pp. 180-181

\begin{tabular}{|l|c|c|c|}
\hline $\begin{array}{l}\text { Position relative to drip } \\
\text { impact }\end{array}$ & $\mathbf{3 3}^{\mathbf{0}}$ & $\begin{array}{c}\text { Transition } \\
\text { (Curved to vertical) }\end{array}$ & $\begin{array}{c}\text { Vertical } \\
\text { (Top of patch on vertical) }\end{array}$ \\
\hline Right (East) $(\mathrm{cm})$ & 9 & 14 & 10 \\
\hline Left (West) $(\mathrm{cm})$ & 6 & 8 & 2.5 \\
\hline
\end{tabular}

Test Description: High Flow Rate $\left(0.2 \mathrm{~m}^{3} / \mathrm{yr}\right.$ nominal $)$, Rough DS Surface

$27 \mathrm{~cm}$ right of drip shield center, $33^{0}$

Scientific Notebook SN-M\&O-SCI-043-V2, pp. 182-183

\begin{tabular}{|l|c|c|c|}
\hline $\begin{array}{l}\text { Position relative to drip } \\
\text { impact }\end{array}$ & $\mathbf{3 3}^{\mathbf{0}}$ & $\begin{array}{c}\text { Transition } \\
\text { (Curved to vertical) }\end{array}$ & $\begin{array}{c}\text { Vertical } \\
\text { (Top of patch on vertical) }\end{array}$ \\
\hline Right $($ East) $(\mathrm{cm})$ & 3 & 11 & 11 \\
\hline Left $($ West) $(\mathrm{cm})$ & 5 & 12 & 13 \\
\hline
\end{tabular}

Test Description: Low Flow Rate $\left(0.2 \mathrm{~m}^{3} / \mathrm{yr}\right.$ nominal $)$, Rough DS Surface

$27 \mathrm{~cm}$ right of drip shield center, $33^{\circ}$

Scientific Notebook SN-M\&O-SCI-043-V2, pp. 184-185

\begin{tabular}{|l|c|c|c|}
\hline $\begin{array}{l}\text { Position relative to drip } \\
\text { impact }\end{array}$ & $\mathbf{3 3}^{\mathbf{0}}$ & $\begin{array}{c}\text { Transition } \\
\text { (Curved to vertical) }\end{array}$ & $\begin{array}{c}\text { Vertical } \\
\text { (Top of patch on vertical) }\end{array}$ \\
\hline Right (East) $(\mathrm{cm})$ & N/A & 2.5 & 3 \\
\hline Left $($ West) $(\mathrm{cm})$ & N/A & 2.5 & 5 \\
\hline
\end{tabular}


Test Description: High Flow Rate $\left(0.2 \mathrm{~m}^{3} / \mathrm{yr}\right.$ nominal), Rough DS Surface $27 \mathrm{~cm}$ right of drip shield center, $16.5^{0}$

Scientific Notebook SN-M\&O-SCI-043-V2, pp. 186-187

\begin{tabular}{|l|c|c|c|}
\hline $\begin{array}{l}\text { Position relative to drip } \\
\text { impact }\end{array}$ & $\mathbf{3 3}^{\mathbf{0}}$ & $\begin{array}{c}\text { Transition } \\
\text { (Curved to vertical) }\end{array}$ & $\begin{array}{c}\text { Vertical } \\
\text { (Top of patch on vertical) }\end{array}$ \\
\hline Right (East) $(\mathrm{cm})$ & 15 & 10 & 10 \\
\hline Left (West) $(\mathrm{cm})$ & 16 & 17 & 20 \\
\hline
\end{tabular}

Test Description: High Flow Rate $\left(0.2 \mathrm{~m}^{3} / \mathrm{yr}\right.$ nominal), Rough DS Surface $27 \mathrm{~cm}$ left of drip shield center, $16.5^{\circ}$

Scientific Notebook SN-M\&O-SCI-043-V2, pp. 188-189

\begin{tabular}{|l|c|c|c|}
\hline $\begin{array}{l}\text { Position relative to drip } \\
\text { impact }\end{array}$ & $\mathbf{3 3}^{\mathbf{0}}$ & $\begin{array}{c}\text { Transition } \\
\text { (Curved to vertical) }\end{array}$ & $\begin{array}{c}\text { Vertical } \\
\text { (Top of patch on vertical) }\end{array}$ \\
\hline Right (East) $(\mathrm{cm})$ & 32 & 34 & 23 \\
\hline Left (West) $(\mathrm{cm})$ & 26 & 13 & 12 \\
\hline
\end{tabular}

Test Description: High Flow Rate $\left(0.2 \mathrm{~m}^{3} / \mathrm{yr}\right.$ nominal), Rough DS Surface

$54 \mathrm{~cm}$ left of drip shield center, $16.5^{0}$

Scientific Notebook SN-M\&O-SCI-043-V2, pp. 190-191

\begin{tabular}{|l|c|c|c|}
\hline $\begin{array}{l}\text { Position relative to drip } \\
\text { impact }\end{array}$ & $\mathbf{3 3}^{\mathbf{0}}$ & $\begin{array}{c}\text { Transition } \\
\text { (Curved to vertical) }\end{array}$ & $\begin{array}{c}\text { Vertical } \\
\text { (Top of patch on vertical) }\end{array}$ \\
\hline Right (East) $(\mathrm{cm})$ & 20 & 19 & 21 \\
\hline Left (West) $(\mathrm{cm})$ & 25 & 26 & 26 \\
\hline
\end{tabular}

Test Description: Low Flow Rate $\left(0.2 \mathrm{~m}^{3} / \mathrm{yr}\right.$ nominal $)$, Rough DS Surface

$54 \mathrm{~cm}$ left of drip shield center, $16.5^{0}$

Scientific Notebook SN-M\&O-SCI-043-V2, pp. 192-193

\begin{tabular}{|l|c|c|c|}
\hline $\begin{array}{l}\text { Position relative to drip } \\
\text { impact }\end{array}$ & $\mathbf{3 3}^{\mathbf{0}}$ & $\begin{array}{c}\text { Transition } \\
\text { (Curved to vertical) }\end{array}$ & $\begin{array}{c}\text { Vertical } \\
\text { (Top of patch on vertical) }\end{array}$ \\
\hline Right (East) $(\mathrm{cm})$ & 6 & N/A & N/A \\
\hline Left (West) $(\mathrm{cm})$ & 3 & N/A & N/A \\
\hline
\end{tabular}

Test Description: High Flow Rate $\left(0.2 \mathrm{~m}^{3} / \mathrm{yr}\right.$ nominal $)$, Rough DS Surface

$54 \mathrm{~cm}$ left of drip shield center, crown

Scientific Notebook SN-M\&O-SCI-043-V2, pp. 194-195

\begin{tabular}{|l|c|c|c|}
\hline $\begin{array}{l}\text { Position relative to drip } \\
\text { impact }\end{array}$ & $\mathbf{3 3}^{\mathbf{0}}$ & $\begin{array}{c}\text { Transition } \\
\text { (Curved to vertical) }\end{array}$ & $\begin{array}{c}\text { Vertical } \\
\text { (Top of patch on vertical) }\end{array}$ \\
\hline Right (East) $(\mathrm{cm})$ & None & N/A & N/A \\
\hline Left (West) $(\mathrm{cm})$ & 2 & N/A & N/A \\
\hline
\end{tabular}

Test Description: High Flow Rate $\left(0.2 \mathrm{~m}^{3} / \mathrm{yr}\right.$ nominal), Rough DS Surface

$27 \mathrm{~cm}$ left of drip shield center, crown

Scientific Notebook SN-M\&O-SCI-043-V2, pp. 196-197

\begin{tabular}{|l|l|l|l|}
\hline $\begin{array}{l}\text { Position relative to drip } \\
\text { impact }\end{array}$ & $\mathbf{3 3}^{\mathbf{0}}$ & $\begin{array}{l}\text { Transition } \\
\text { (Curved to vertical) }\end{array}$ & $\begin{array}{l}\text { Vertical } \\
\text { (Top of patch on vertical) }\end{array}$ \\
\hline Right (East) $(\mathrm{cm})$ & 15 & 15 & 15 \\
\hline Left (West) $(\mathrm{cm})$ & 15 & 14 & 10 \\
\hline
\end{tabular}


Test Description: High Flow Rate $\left(0.2 \mathrm{~m}^{3} / \mathrm{yr}\right.$ nominal), Rough DS Surface

$27 \mathrm{~cm}$ right of drip shield center, crown

Scientific Notebook SN-M\&O-SCI-043-V2, pp. 198-199

\begin{tabular}{|l|c|c|c|}
\hline $\begin{array}{l}\text { Position relative to drip } \\
\text { impact }\end{array}$ & $\mathbf{3 3}^{\mathbf{0}}$ & $\begin{array}{c}\text { Transition } \\
\text { (Curved to vertical) }\end{array}$ & $\begin{array}{c}\text { Vertical } \\
\text { (Top of patch on vertical) }\end{array}$ \\
\hline Right (East) $(\mathrm{cm})$ & 6 & 3 & 4 \\
\hline Left (West) $(\mathrm{cm})$ & 6 & 3 & 2 \\
\hline
\end{tabular}

Test Description: Low Flow Rate $\left(0.2 \mathrm{~m}^{3} / \mathrm{yr}\right.$ nominal $)$, Rough DS Surface

$27 \mathrm{~cm}$ right of drip shield center, crown

Scientific Notebook SN-M\&O-SCI-043-V2, pp. 204-205

\begin{tabular}{|l|c|c|c|}
\hline $\begin{array}{l}\text { Position relative to drip } \\
\text { impact }\end{array}$ & $\mathbf{3 3}^{\mathbf{0}}$ & $\begin{array}{c}\text { Transition } \\
\text { (Curved to vertical) }\end{array}$ & $\begin{array}{c}\text { Vertical } \\
\text { (Top of patch on vertical) }\end{array}$ \\
\hline Right (East) $(\mathrm{cm})$ & 16.0 & Enter B5 & Enter B5 \\
\hline Left (West) $(\mathrm{cm})$ & 50.0 & 53.0 & 52.0 \\
\hline
\end{tabular}

Test Description: Low Flow Rate $\left(0.2 \mathrm{~m}^{3} / \mathrm{yr}\right.$ nominal $)$, Rough DS Surface

$27 \mathrm{~cm}$ right of drip shield center, crown

Scientific Notebook SN-M\&O-SCI-043-V2, pp. 206-207

\begin{tabular}{|l|c|c|c|}
\hline $\begin{array}{l}\text { Position relative to drip } \\
\text { impact }\end{array}$ & $\mathbf{3 3}^{\mathbf{0}}$ & $\begin{array}{c}\text { Transition } \\
\text { (Curved to vertical) }\end{array}$ & $\begin{array}{c}\text { Vertical } \\
\text { (Top of patch on vertical) }\end{array}$ \\
\hline Right (East) $(\mathrm{cm})$ & 1.0 & $\mathrm{~N} / \mathrm{A}$ & N/A \\
\hline Left $($ West $)(\mathrm{cm})$ & N/A & 4.0 & 3.5 \\
\hline
\end{tabular}

Test Description: Low Flow Rate $\left(0.2 \mathrm{~m}^{3} / \mathrm{yr}\right.$ nominal), Rough DS Surface

$27 \mathrm{~cm}$ left of drip shield center, crown

Scientific Notebook SN-M\&O-SCI-043-V2, pp. 208-209

\begin{tabular}{|l|c|c|c|}
\hline $\begin{array}{l}\text { Position relative to drip } \\
\text { impact }\end{array}$ & $\mathbf{3 3}^{\mathbf{0}}$ & $\begin{array}{c}\text { Transition } \\
\text { (Curved to vertical) }\end{array}$ & $\begin{array}{c}\text { Vertical } \\
\text { (Top of patch on vertical) }\end{array}$ \\
\hline Right (East) $(\mathrm{cm})$ & 12.0 & 16.0 & 18.0 \\
\hline Left (West) $(\mathrm{cm})$ & 25.5 & Enter B4 & Enter B4 \\
\hline
\end{tabular}

Test Description: Low Flow Rate $\left(0.2 \mathrm{~m}^{3} / \mathrm{yr}\right.$ nominal $)$, Rough DS Surface

$54 \mathrm{~cm}$ left of drip shield center, crown

Scientific Notebook SN-M\&O-SCI-043-V2, pp. 210-211

\begin{tabular}{|l|c|c|c|}
\hline $\begin{array}{l}\text { Position relative to drip } \\
\text { impact }\end{array}$ & $\mathbf{3 3}^{\mathbf{0}}$ & $\begin{array}{c}\text { Transition } \\
\text { (Curved to vertical) }\end{array}$ & $\begin{array}{c}\text { Vertical } \\
\text { (Top of patch on vertical) }\end{array}$ \\
\hline Right (East) $(\mathrm{cm})$ & 0 & 4.5 & 5.0 \\
\hline Left $($ West) $(\mathrm{cm})$ & 0 & N/A & N/A \\
\hline
\end{tabular}

Test Description: Low Flow Rate $\left(0.2 \mathrm{~m}^{3} / \mathrm{yr}\right.$ nominal), Rough DS Surface

$27 \mathrm{~cm}$ left of drip shield center, $16.5^{0}$

Scientific Notebook SN-M\&O-SCI-043-V2, pp. 212-213

\begin{tabular}{|l|c|c|c|}
\hline $\begin{array}{l}\text { Position relative to drip } \\
\text { impact }\end{array}$ & $\mathbf{3 3}^{\mathbf{0}}$ & $\begin{array}{c}\text { Transition } \\
\text { (Curved to vertical) }\end{array}$ & $\begin{array}{c}\text { Vertical } \\
\text { (Top of patch on vertical) }\end{array}$ \\
\hline Right (East) $(\mathrm{cm})$ & 2.0 & 0 & 2.5 \\
\hline Left (West) $(\mathrm{cm})$ & 3.0 & 1.0 & 1.0 \\
\hline
\end{tabular}


Test Description: Low Flow Rate $\left(0.2 \mathrm{~m}^{3} / \mathrm{yr}\right.$ nominal), Rough DS Surface $27 \mathrm{~cm}$ right of drip shield center, $16.5^{0}$

Scientific Notebook SN-M\&O-SCI-043-V2, pp. 214-215

\begin{tabular}{|l|c|c|c|}
\hline $\begin{array}{l}\text { Position relative to drip } \\
\text { impact }\end{array}$ & $\mathbf{3 3}^{\mathbf{0}}$ & $\begin{array}{c}\text { Transition } \\
\text { (Curved to vertical) }\end{array}$ & $\begin{array}{c}\text { Vertical } \\
\text { (Top of patch on vertical) }\end{array}$ \\
\hline Right (East) $(\mathrm{cm})$ & 0 & 0 & 0 \\
\hline Left $($ West) $(\mathrm{cm})$ & 0 & 0 & 0 \\
\hline
\end{tabular}

Test Description: Low Flow Rate $\left(0.2 \mathrm{~m}^{3} / \mathrm{yr}\right.$ nominal $)$, Rough DS Surface

$27 \mathrm{~cm}$ left of drip shield center, $33^{0}$

Scientific Notebook SN-M\&O-SCI-043-V2, pp. 216-217

\begin{tabular}{|l|c|c|c|}
\hline $\begin{array}{l}\text { Position relative to drip } \\
\text { impact }\end{array}$ & $\mathbf{3 3}^{\mathbf{0}}$ & $\begin{array}{c}\text { Transition } \\
\text { (Curved to vertical) }\end{array}$ & $\begin{array}{c}\text { Vertical } \\
\text { (Top of patch on vertical) }\end{array}$ \\
\hline Right (East) $(\mathrm{cm})$ & N/A & 4.5 & 4.5 \\
\hline Left $($ West) $(\mathrm{cm})$ & N/A & 6.0 & 9.5 \\
\hline
\end{tabular}




\section{APPENDIX D: Water Temperature Data}

Date: $7 / 22 / 02$

Test: Low Flow Rate $\left(0.2 \mathrm{~m}^{3} / \mathrm{yr}\right.$ nominal), Rough DS Surface

Scientific Notebook SN-M\&O-SCI-043-V2, pp. 204-205

RTD Used: DS-RTD-01with Fluke 702 multimeter in RTD temperature mode

(See Page 203 of SN-M\&0-SCI-043-V2)

\begin{tabular}{|c|c|c|c|}
\hline Measurement & Temperature $\left({ }^{\circ} \mathrm{C}\right)$ & Ohms & Time \\
\hline Input Water & 28.5 & 111.1 & $9: 11$ \\
\hline Input Water & 33.4 & 113.0 & $13: 52$ \\
\hline DS Beaker Water & 32.1 & 112.4 & $13: 56$ \\
\hline
\end{tabular}

Date: $7 / 22 / 02$

Test: Low Flow Rate ( $0.2 \mathrm{~m} 3 / \mathrm{yr}$ nominal), Rough DS Surface

Scientific Notebook SN-M\&O-SCI-043-V2, pp. 206-207

RTD Used: DS-RTD-01with Fluke 702 multimeter in RTD temperature mode (See Page 203 of SN-M\&0-SCI-043-V2)

\begin{tabular}{|c|c|c|c|}
\hline Measurement & Temperature $\left({ }^{\circ} \mathrm{C}\right)$ & Ohms & Time \\
\hline Input Water & 31.2 & 112.1 & $14: 47$ \\
\hline DS Beaker Water & 30.5 & 111.3 & $14: 58$ \\
\hline DS Surface Water & 31.9 & 112.4 & $20: 01$ \\
\hline DS Beaker Water & 31.6 & 112.4 & $20: 06$ \\
\hline Input Water & 33.9 & 113.2 & $20: 04$ \\
\hline
\end{tabular}

Date: $7 / 23 / 02$

Test: Low Flow Rate ( $0.2 \mathrm{~m} 3 / \mathrm{yr}$ nominal), Rough DS Surface

Scientific Notebook SN-M\&O-SCI-043-V2, pp. 208-209

RTD Used: DS-RTD-01with Fluke 702 multimeter in RTD temperature mode

(See Page 203 of SN-M\&0-SCI-043-V2)

\begin{tabular}{|c|c|c|c|}
\hline Measurement & Temperature $\left({ }^{\circ} \mathrm{C}\right)$ & Ohms & Time \\
\hline Input Water & 23.9 & 109.3 & $7: 08$ \\
\hline DS Beaker Water & 22.1 & 108.4 & $7: 14$ \\
\hline DS Beaker Water & 29.4 & 111.4 & $12: 06$ \\
\hline DS Surface Water & 30.1 & 111.7 & $12: 23$ \\
\hline Input Water & 30.0 & 111.7 & $12: 27$ \\
\hline
\end{tabular}


Date: $7 / 23 / 02$

Test: Low Flow Rate ( $0.2 \mathrm{~m} 3 / \mathrm{yr}$ nominal), Rough DS Surface

Scientific Notebook SN-M\&O-SCI-043-V2, pp. 210-211

RTD Used: DS-RTD-01with Fluke 702 multimeter in RTD temperature mode

(See Page 203 of SN-M\&0-SCI-043-V2)

\begin{tabular}{|c|c|c|c|}
\hline Measurement & Temperature $\left({ }^{\circ} \mathrm{C}\right)$ & Ohms & Time \\
\hline Input Water & 30.4 & 111.8 & $12: 53$ \\
\hline DS Beaker Water & 29.8 & 111.6 & $12: 54$ \\
\hline DS Beaker Water & 28.3 & 111.0 & $18: 02$ \\
\hline Input Water & 31.5 & 112.3 & $18: 06$ \\
\hline DS Surface Water & 30.1 & 111.7 & $18: 12$ \\
\hline
\end{tabular}

Date: $7 / 24 / 02$

Test: Low Flow Rate ( $0.2 \mathrm{~m} 3 / \mathrm{yr}$ nominal), Rough DS Surface

Scientific Notebook SN-M\&O-SCI-043-V2, pp. 212-213

RTD Used: DS-RTD-01with Fluke 702 multimeter in RTD temperature mode

(See Page 203 of SN-M\&0-SCI-043-V2)

\begin{tabular}{|c|c|c|c|}
\hline Measurement & Temperature $\left({ }^{\circ} \mathrm{C}\right)$ & Ohms & Time \\
\hline DS Beaker Water & 21.2 & 108.3 & $7: 04$ \\
\hline Input Water & 23.1 & 109.0 & $7: 06$ \\
\hline DS Surface Water & 30.7 & 112.0 & $12: 23$ \\
\hline DS Beaker Water & 29.8 & 111.6 & $12: 28$ \\
\hline Input Water & 29.9 & 111.7 & $12: 36$ \\
\hline
\end{tabular}

Date: $7 / 24 / 02$

Test: Low Flow Rate ( $0.2 \mathrm{~m} 3 / \mathrm{yr}$ nominal), Rough DS Surface

Scientific Notebook SN-M\&O-SCI-043-V2, pp. 214-215

RTD Used: DS-RTD-01with Fluke 702 multimeter in RTD temperature mode

(See Page 203 of SN-M\&0-SCI-043-V2)

\begin{tabular}{|l|l|l|l|}
\hline Measurement & Temperature $\left({ }^{\circ} \mathrm{C}\right)$ & Ohms & Time \\
\hline Input Water & 30.2 & 111.7 & $12: 51$ \\
\hline DS Beaker Water & 30.0 & 111.7 & $12: 53$ \\
\hline Air Temperature & 30.3 & 111.8 & $12: 59$ \\
\hline Air Temperature & 31.9 & 112.4 & $18: 00$ \\
\hline DS Beaker Water & 31.6 & 112.3 & $18: 02$ \\
\hline DS Surface Water & 32.1 & 112.4 & $18: 06$ \\
\hline Input Water & 33.5 & 113.0 & $18: 12$ \\
\hline
\end{tabular}


Date: $7 / 25 / 02$

Test: Low Flow Rate $\left(0.2 \mathrm{~m}^{3} / \mathrm{yr}\right.$ nominal), Rough DS Surface

Scientific Notebook SN-M\&O-SCI-043-V2, pp. 216-217

\begin{tabular}{|c|c|c|c|}
\hline \multicolumn{4}{|c|}{$\begin{array}{l}\text { RTD Used: DS-RTD-01with Fluke } 702 \text { multimeter in RTD temperature mode } \\
\text { (See Page } 203 \text { of SN-M\&0-SCI-043-V2) }\end{array}$} \\
\hline Measurement & Temperature $\left({ }^{\circ} \mathrm{C}\right)$ & Ohms & Time \\
\hline Input Water & 22.6 & 108.8 & $7: 28$ \\
\hline DS Beaker Water & 22.2 & 108.6 & $7: 36$ \\
\hline Air Temperature & 23.6 & 109.1 & $7: 38$ \\
\hline Air Temperature & 30.2 & 111.8 & $12: 37$ \\
\hline DS Surface Water & 30.0 & 111.7 & $12: 42$ \\
\hline DS Beaker Water & 29.5 & 111.5 & $12: 48$ \\
\hline Input Water & 29.3 & 111.4 & $12: 50$ \\
\hline
\end{tabular}

Description of Measurement Types

Input Water:

Injection source water in beaker located on Analytical Balance PM4000 (see page 3 of SN-M\&O-SCI-043-V2) inside the test chamber. RTD probe submerged in water and reading was taken after $30 \mathrm{sec}$.

DS Beaker Water: Water in glass beaker placed in contact with the DS surface throughout the test. RTD probe is placed below water surface in beaker and reading was taken after $30 \mathrm{sec}$.

DS Surface Water: Water film remaining on the DS following a test. RTD probe is placed in contact with the DS. The probe is not submerged in water, and in most cases only a thin film is present. Reading is taken after $30 \mathrm{sec}$.

Air Temperature: RTD probe is mounted so that it is not in contact with DS any other surface. Reading is taken after $30 \mathrm{sec}$. 